MÉMOIRES DE LA SMF 84

\title{
THÉORIE D'IWASAWA DES REPRÉSENTATIONS $p$-ADIQUES SEMI-STABLES
}

Bernadette Perrin-Riou

Société Mathématique de France 2001

Publié avec le concours du Centre National de la Recherche Scientifique 


\section{B. Perrin-Riou}

Mathématiques, UMR 8628 du CNRS, Université Paris-Sud, F-91405 Orsay Cedex, France.

E-mail : bpr@math.u-psud.fr

Classification mathématique par sujets (2000). - 11E95, 11R23.

Mots clefs. - Semi-stable, Iwasawa, normes universelles. 


\title{
THÉORIE D'IWASAWA DES REPRÉSENTATIONS $p$-ADIQUES SEMI-STABLES
}

\author{
Bernadette Perrin-Riou
}

Résumé. - Soient $F$ une extension finie non ramifiée de $\mathbb{Q}_{p}$ et $V$ une représentation $p$-adique galoisienne semi-stable sur $F$ de dimension $d$. On développe dans ce texte la théorie d'Iwasawa relative à $V$ et à la $\mathbb{Z}_{p}$-extension cyclotomique. En particulier, on construit un «logarithme » (régulateur) du module d'Iwasawa local associé à $V$ (construit à partir de sa cohomologie galoisienne) dans un module très explicite sur l'algèbre engendrée par les fonctions analytiques sur la couronne $\left\{p^{-1 /(p-1)}<|x|<1\right\}$ et $\log x$.

\section{Abstract (Iwasawa theory of semi-stable $p$-adic representations)}

Let $F$ be a finite unramified extension of $\mathbb{Q}_{p}$ and $V$ a $p$-adic galois semi-stable representation on $F$ of dimension $d$. We develop Iwasawa theory for $V$ and the $\mathbb{Z}_{p^{-}}$ cyclotomic extension: we construct a logarithm (regulator map) from the Iwasawa module associated to the Galois cohomology of $V$ in a very explicit module on an algebra generated by analytic functions on the annulus $\left\{p^{-1 /(p-1)}<|x|<1\right\}$ and $\log x$. 



\section{TABLE DES MATIÈRES}

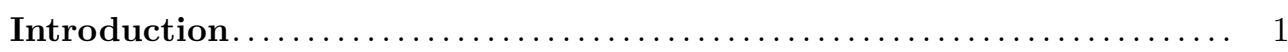

1. Anneaux de fonctions $\ldots \ldots \ldots \ldots \ldots \ldots \ldots \ldots \ldots \ldots \ldots \ldots \ldots \ldots \ldots$

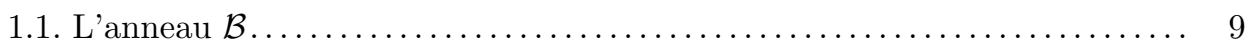

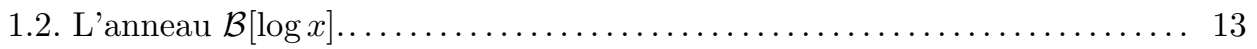

1.3. Structure de $G_{\infty}$-modules............................. 19

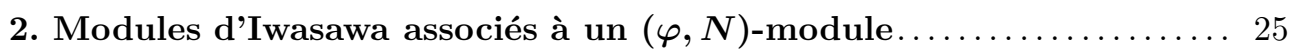

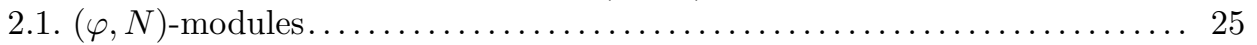

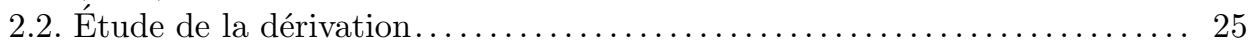

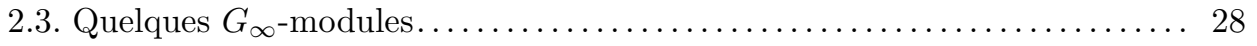

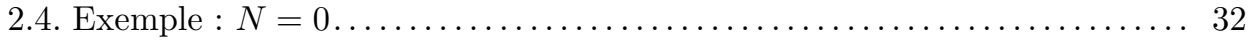

2.5. Exemples de dimension $\leqslant 2 \ldots \ldots \ldots \ldots \ldots \ldots \ldots \ldots \ldots \ldots \ldots \ldots \ldots \ldots \ldots \ldots \ldots$

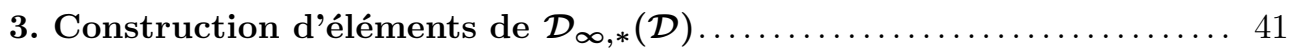

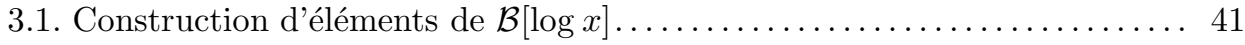

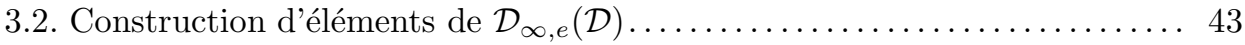

3.3. Construction d'éléments de $\mathcal{D}_{\infty, g}(\mathcal{D}) \ldots \ldots \ldots \ldots \ldots \ldots \ldots \ldots \ldots \ldots \ldots$

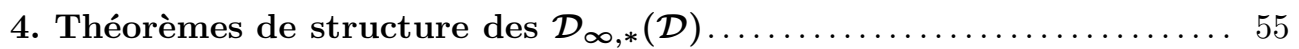

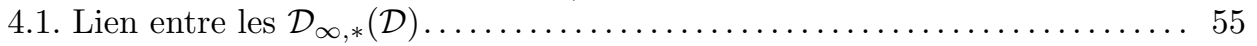

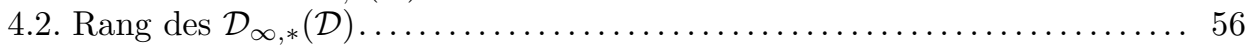

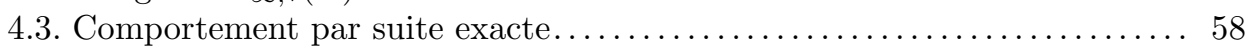

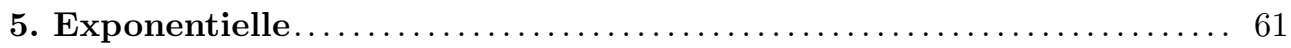

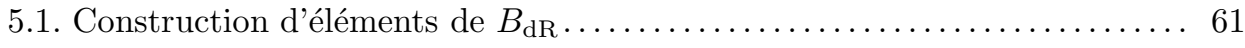

5.2 . Théorème-définition de l'exponentielle...................... 69

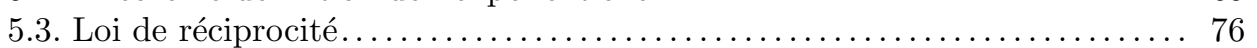

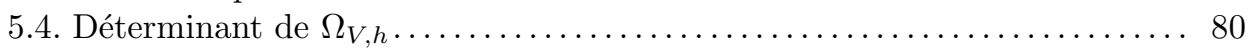




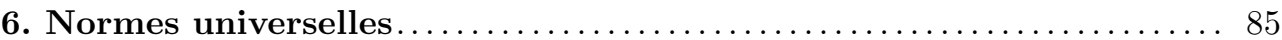

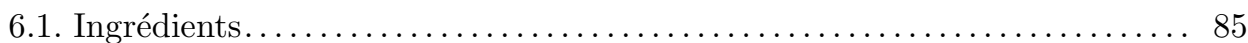

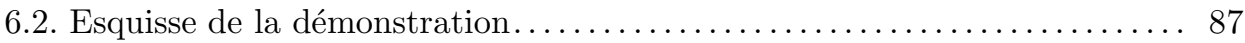

A. Digression : le polylogarithme.......................... 89

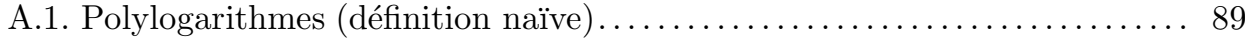

A.2. Polylogarithmes et fonction de Kubota-Leopoldt. ................. 93

A.3. Polylogarithmes et éléments de $B_{\text {st }}^{+G_{K_{\infty}} \ldots \ldots \ldots \ldots \ldots \ldots \ldots \ldots \ldots \ldots \ldots \ldots \ldots \ldots \ldots}$

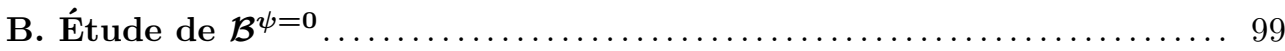

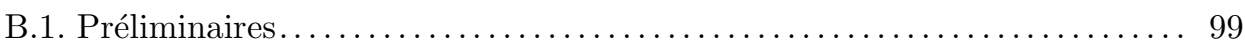

B.2. Structure de $G_{\infty}$-modules............................. 99

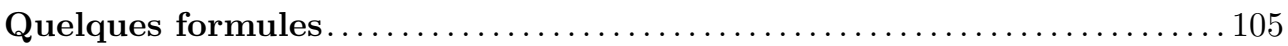

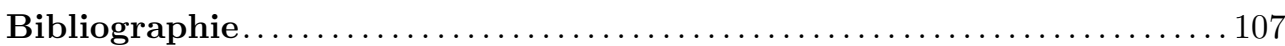

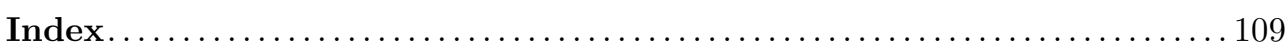




\section{INTRODUCTION}

Ce texte fait partie d'une série d'articles désirant comprendre la théorie d'Iwasawa des représentations $p$-adiques géométriques sur une extension finie $K$ de $\mathbb{Q}_{p}$ ainsi que de faire une théorie générale des fonctions $L p$-adiques associées à une représentation $p$-adique géométrique d'un corps de nombres. Dans [19] a d'abord été traité le cas des représentations $p$-adiques absolument cristallines (cristallines sur un corps $K$ non ramifié). Ce travail local a été ensuite utilisé dans [21] dans le cas des représentations $p$-adiques géométriques du groupe de Galois absolu $G_{\mathbb{Q}}$ de $\mathbb{Q}$ qui sont cristallines en $p$. Des cas particuliers exploitant ce travail ont été traités dans [20] (cas multiplicatif), [22] (cas des courbes elliptiques), [24] (cas du carré symétrique d'une courbe elliptique).

Dans [6], Colmez a montré la loi de réciprocité conjecturée dans [19], ce qui est fondamental, car une partie des résultats des articles cités reposait sur cette loi. De plus, il étend en partie la théorie faite dans le cas des représentations $p$-adiques de de Rham. Cependant, ce travail me semblait inachevé et peu exploitable (par moi en tout cas) tel quel.

Dans le texte qui suit, nous construisons, dans le cas des représentations $p$-adiques semi-stables d'une extension finie non ramifiée de $\mathbb{Q}_{p}$, l'exponentielle et le logarithme « élargis » en théorie d'Iwasawa. Nous démontrons, pour les représentations semistables, la conjecture énoncée dans [26] relative au rang des normes universelles. Du travail de Colmez, nous utilisons la loi de réciprocité.

Expliquons la manière de procéder en prenant $K=\mathbb{Q}_{p}$ :

1) Soit $\mathcal{H}$ l'algèbre des fonctions (strictement) analytiques sur le disque $\left\{x \in \mathbb{C}_{p},|x|<1\right\}$ vérifiant une condition de croissance logarithmique lorsque $|x| \rightarrow 1$. Soit $\mathcal{B}$ l'algèbre des fonctions (strictement) analytiques sur une couronne du type $\left\{x \in \mathbb{C}_{p}, \eta \leqslant|x|<1\right\}$ et vérifiant là encore une condition de croissance logarithmique lorsque $|x| \rightarrow 1$. Choisissons un(e branche du) logarithme $\log x$, c'est-à-dire une fonction localement analytique sur $\mathbb{C}_{p}-\{0\}$ vérifiant l'équation fonctionnelle 
$\log x y=\log x+\log y$ et dont la dérivée en 1 soit 1 . L'algèbre $\mathcal{B}[\log x]$ des polynômes en $\log x$ à coefficients dans $\mathcal{B}$ peut être munie d'une action du groupe de Galois $G_{\infty}=\operatorname{Gal}\left(\mathbb{Q}_{p}\left(\mu_{p \infty}\right) / \mathbb{Q}_{p}\right)$, d'un opérateur $\varphi$ (opérateur de Frobenius) et d'un opérateur $N$ (opérateur de monodromie) commutant à l'action de $G_{\infty}$ et vérifiant $N \varphi=p \varphi N$ et d'une dérivation $D$ prolongeant les actions usuelles sur $\mathcal{B}$, commutant avec $N$ et telle que $D \varphi=p \varphi D$ et $D \tau=\chi(\tau) \tau D$ pour $\tau \in G_{\infty}$ et $\chi$ le caractère cyclotomique donnant l'action de $G_{\infty}$ sur les racines de l'unité d'ordre une puissance de $p$. Enfin $\varphi$ admet un inverse à gauche $\psi$. Ainsi, pour $f \in \mathcal{B}[\log x]$

$$
\varphi \circ \psi(f)=p^{-1} \sum_{\zeta \in \mu_{p}} f(\zeta(1+x)-1)
$$

où $\mu_{p}$ est le groupe des racines $p$-ièmes de l'unité. Soit $\Gamma=\operatorname{Gal}\left(\mathbb{Q}_{p}\left(\mu_{p^{\infty}}\right) / \mathbb{Q}_{p}\left(\mu_{p}\right)\right)$ et $\Delta=\operatorname{Gal}\left(\mathbb{Q}_{p}\left(\mu_{p}\right) / \mathbb{Q}_{p}\right)$. Définissons

$$
\mathcal{B}\left(G_{\infty}\right)=\mathbb{Z}_{p}[\Delta] \otimes \mathcal{B}(\Gamma), \quad \mathcal{H}\left(G_{\infty}\right)=\mathbb{Z}_{p}[\Delta] \otimes \mathcal{H}(\Gamma)
$$

avec $\mathcal{B}(\Gamma)$ (resp. $\mathcal{H}(\Gamma)$ ) l'image de $\mathcal{B}$ (resp. de $\mathcal{H}$ ) par l'application $x \mapsto \gamma-1$ où $\gamma$ est un générateur topologique de $\Gamma$. Les anneaux $\mathcal{B}$ et $\mathcal{H}, \mathcal{B}(\Gamma)$ et $\mathcal{H}(\Gamma)$ sont intègres. On utilise la notion suivante de rang d'un module : le rang d'un module $M$ sur un anneau intègre $A$ est la dimension de $K \otimes_{A} M$ sur $K$ pour $K$ le corps de fractions de $A$. Un $\mathcal{H}\left(G_{\infty}\right)$-module $M$ est dit de torsion s'il est de torsion en tant que $\mathcal{H}(\Gamma)$-module, on dit qu'il est de rang $r$ si ses composantes sous l'action de $\Delta$ sont toutes de rang $r$ sur $\mathcal{H}(\Gamma)$.

En utilisant un résultat de Cherbonnier et Colmez $[\mathbf{3}]$, on démontre que $\mathcal{B}[\log x]^{\psi=0}$ est muni d'une structure naturelle de $\mathcal{B}\left(G_{\infty}\right)$-module libre et admet comme base les $(1+x) p^{-i} \varphi \log ^{i} x$ pour $i$ entier $\geqslant 0$.

2) Soit $\mathcal{D}$ un $(\varphi, N)$-module de dimension finie sur $\mathbb{Q}_{p}$, c'est-à-dire un $\mathbb{Q}_{p}$-espace vectoriel de dimension finie muni d'un opérateur bijectif $\varphi$ (Frobenius) et d'un opérateur $N$ (monodromie) vérifiant la relation $N \varphi=p \varphi N$. Soit

$$
\mathcal{D}_{\infty}^{\text {gros }}(\mathcal{D})=\left(\mathcal{B}[\log x]^{\psi=0} \otimes \mathcal{D}\right)^{N=0}
$$

C'est un $\mathcal{B}\left(G_{\infty}\right)$-module de rang $\operatorname{dim}_{\mathbb{Q}_{p}} \mathcal{D}$ : il existe une $\mathbb{Q}_{p}$-application linéaire naturelle de $\mathcal{D}$ dans $\mathcal{D}_{\infty}^{\text {gros }}(\mathcal{D})$ donnée par

$$
d \longmapsto d^{\mu}=(1+x) \exp \left(-p^{-1} \varphi \log x \cdot N\right) d
$$

et $\mathcal{D}_{\infty}^{\text {gros }}(\mathcal{D}) \cong \mathcal{B}\left(G_{\infty}\right) \mathcal{D}^{\mu}$.

Pour $* \in\{e, f, g\}$, nous définissons des sous-modules $\mathcal{D}_{\infty, *}(\mathcal{D})$ de $\mathcal{D}_{\infty}^{\text {gros }}(\mathcal{D})$ sur $\mathcal{H}\left(G_{\infty}\right)$ de rang $\operatorname{dim}_{\mathbb{Q}_{p}} \mathcal{D}$. Par exemple, si le noyau de $\varphi-p^{j}$ sur $\mathcal{D}$ est nul pour tout entier $j$, les $\mathcal{D}_{\infty, *}(\mathcal{D})$ sont tous égaux au sous-module des éléments de $\mathcal{D}_{\infty}^{\text {gros }}(\mathcal{D})$ qui appartiennent à l'image de $(\mathcal{B}[\log x] \otimes \mathcal{D})^{N=0}$ par $1-\Phi$, c'est-à-dire à

$$
(1-\Phi)(\mathcal{B}[\log x] \otimes \mathcal{D})^{\psi=1 \otimes \varphi, N=0}
$$

(ici, $\Phi=\varphi \otimes \varphi$ et $N=1 \otimes N+N \otimes 1$ ). 
Les lettres $e, f$ et $g$ ont bien sûr un rapport avec les $e, f$ et $g$ apparaissant dans les définitions de Bloch et Kato [2] concernant certains sous-groupes de cohomologie galoisienne.

ThÉORÈme A. - Les $\mathcal{H}\left(G_{\infty}\right)$-modules $\mathcal{D}_{\infty, *}(\mathcal{D})$ sont de type fini, sans torsion et de rang égal à $\operatorname{dim}_{\mathbb{Q}_{p}} \mathcal{D}$. De plus, ils admettent une résolution de longueur finie par des modules projectifs de type fini.

On peut définir le déterminant de tels $\mathcal{H}\left(G_{\infty}\right)$-modules ([16], dans la catégorie dérivée des $\mathcal{H}\left(G_{\infty}\right)$-modules, ils sont représentés par un complexe parfait). Nous ne considérerons ici que les déterminants au signe près. Nous examinons aussi les relations entre les modules $\mathcal{D}_{\infty, *}(\mathcal{D})$. Soit $\mathcal{U}(\mathcal{D})=\oplus_{i \in \mathbb{Z}} \mathcal{D}[i](i)$ où $\mathcal{D}[i](i)$ est l'espace vectoriel $\mathcal{D}$ muni de l'opérateur de monodromie de $\mathcal{D}$, de l'opérateur de Frobenius donné par $p^{i} \varphi$ et de l'action de Galois donnée par le caractère $\chi^{i}$. On a des suites exactes naturelles de $G_{\infty}$-modules

$$
\begin{gathered}
0 \longrightarrow \mathcal{D}_{\infty, e}(\mathcal{D}) \longrightarrow \mathcal{D}_{\infty, f}(\mathcal{D}) \longrightarrow \mathcal{U}(\mathcal{D})^{N=0} /(1-\Phi) \mathcal{U}(\mathcal{D})^{N=0} \longrightarrow 0 \\
0 \longrightarrow \mathcal{D}_{\infty, f}(\mathcal{D}) \longrightarrow \mathcal{D}_{\infty, g}(\mathcal{D}) \longrightarrow(\mathcal{U}(\mathcal{D}) / N \mathcal{U}(\mathcal{D}))^{\Phi=p^{-1}} \longrightarrow 0 \\
0 \longrightarrow \mathcal{D}_{\infty, e}(\mathcal{D}) \longrightarrow \mathcal{D}_{\infty, g}(\mathcal{D}) \longrightarrow \mathcal{C}_{\infty}(\mathcal{D}) \longrightarrow 0
\end{gathered}
$$

où $\mathcal{C}_{\infty}(\mathcal{D})$ est le premier groupe de cohomologie du complexe

$$
\begin{array}{cccc}
\mathcal{U}(\mathcal{D}) & \longrightarrow \mathcal{U}(\mathcal{D}) \times \mathcal{U}(\mathcal{D}) & \longrightarrow & \mathcal{U}(\mathcal{D}) \\
u & \longmapsto(N u,(1-\Phi) u) & & \\
(u, v) & \longmapsto & N v-(1-p \Phi) u .
\end{array}
$$

Enfin, si l'on pose

$$
\left\{\begin{array}{l}
\mathcal{D}_{\infty, g}^{1}(\mathcal{D})=\mathcal{D}_{\infty, g}(\mathcal{D}) \\
\mathcal{D}_{\infty, g}^{2}(\mathcal{D})=\mathcal{U}(\mathcal{D}) /(N, 1-p \Phi) \mathcal{U}(\mathcal{D}) \\
\mathcal{D}_{\infty, g}^{i}(\mathcal{D})=0 \quad \text { pour } i \neq 1,2,
\end{array}\right.
$$

et si $0 \rightarrow \mathcal{D}_{1} \rightarrow \mathcal{D}_{2} \rightarrow \mathcal{D}_{3} \rightarrow 0$ est une suite exacte de $(\varphi, N)$-modules, on définit une application de connexion $\mathcal{D}_{\infty, g}^{1}\left(\mathcal{D}_{3}\right) \rightarrow \mathcal{D}_{\infty, g}^{2}\left(\mathcal{D}_{1}\right)$ et on a une suite exacte longue

$$
\ldots \longrightarrow \mathcal{D}_{\infty, g}^{i}\left(\mathcal{D}_{1}\right) \longrightarrow \mathcal{D}_{\infty, g}^{i}\left(\mathcal{D}_{2}\right) \longrightarrow \mathcal{D}_{\infty, g}^{i}\left(\mathcal{D}_{3}\right) \longrightarrow \ldots
$$

3) Soit $V$ une représentation $p$-adique semi-stable du groupe de Galois absolu $G_{\mathbb{Q}_{p}}$ de $\mathbb{Q}_{p}$. Il lui est associé par la théorie de Fontaine un $(\varphi, N)$-module filtré $\mathbf{D}_{p}(V)$ et donc un $\mathcal{H}\left(G_{\infty}\right)$-module $\mathcal{D}_{\infty, g}\left(\mathbf{D}_{p}(V)\right)$. Définissons le module d'Iwasawa $Z_{\infty}^{1}\left(\mathbb{Q}_{p}, T\right)$ associé à $V$ et à $\mathbb{Q}_{p}\left(\mu_{p^{\infty}}\right) / \mathbb{Q}_{p}$ comme la limite projective des groupes de cohomologie galoisienne $H^{1}\left(\mathbb{Q}_{p}\left(\mu_{p^{n}}\right), T\right)$ pour $T$ réseau de $V$ stable par $G_{\mathbb{Q}_{p}}$. C'est un $\mathbb{Z}_{p} \llbracket G_{\infty} \rrbracket$ module de type fini de rang $\operatorname{dim} V$ dont le sous-module de torsion est isomorphe à $T^{G_{K_{\infty}}}$. Définissons de même $Z_{\infty}^{2}\left(\mathbb{Q}_{p}, T\right)$ comme la limite projective des groupes de cohomologie $H^{2}\left(\mathbb{Q}_{p}\left(\mu_{p^{n}}\right), T\right)$. Par les théorèmes de dualité de Tate, $Z_{\infty}^{2}\left(\mathbb{Q}_{p}, T\right)$ est 
isomorphe à $T^{*}(1)^{G_{K_{\infty}}}$ à un groupe fini près (voir par exemple [19, 3.2.1]). On note $\widetilde{Z}_{\infty}^{1}\left(\mathbb{Q}_{p}, T\right)=Z_{\infty}^{1}\left(\mathbb{Q}_{p}, T\right) / T^{G_{K_{\infty}}}$.

Le théorème suivant assure l'existence d'une application exponentielle en théorie d'Iwasawa.

THÉorème B.1. - Soit $h$ un entier $\geqslant 1$ tel que $\operatorname{Fil}^{-h} \mathbf{D}_{p}(V)=\mathbf{D}_{p}(V)$. Il existe un homomorphisme naturel injectif de $\mathcal{H}\left(G_{\infty}\right)$-modules

$$
\Omega_{V, h}: \mathcal{D}_{\infty, g}\left(\mathbf{D}_{p}(V)\right) \longrightarrow \mathcal{H}\left(G_{\infty}\right) \otimes_{\mathbb{Z}_{p} \llbracket G_{\infty} \rrbracket} \widetilde{Z}_{\infty}^{1}\left(\mathbb{Q}_{p}, T\right)
$$

Le théorème est bien sûr incomplet. L'application $\Omega_{V, h}$ est caractérisée par une foule de valeurs spéciales reliées aux exponentielles de Bloch-Kato et à leur application duale pour $V$ ou son dual de Tate $V^{*}(1)=\operatorname{Hom}_{\mathbb{Q}_{p}}\left(V, \mathbb{Q}_{p}(1)\right)$ et ses tordues $V(j)=V \otimes \mathbb{Q}_{p}(j)$ (voir 5.3.5).

Posons

$$
\ell_{j}=\frac{\log \gamma}{\log \chi(\gamma)}-j
$$

où $\gamma$ est un élément d'ordre infini de $G_{\infty}$. Notons $\delta_{h}\left(\Omega_{V}\right)$ le sous- $\mathcal{H}\left(G_{\infty}\right)$-module de rang 1 de l'anneau total des fractions $\operatorname{Frac}\left(\mathcal{H}\left(G_{\infty}\right)\right)$ de $\mathcal{H}\left(G_{\infty}\right)$, qui est le déterminant de $\Omega_{V, h}$ sur

$$
\otimes_{i \in\{1,2\}}\left(\operatorname{det} \mathcal{D}_{\infty, g}^{i}\left(\mathbf{D}_{p}(V)\right)\right)^{(-1)^{i}} \otimes_{\mathbb{Z}_{p} \llbracket G_{\infty} \rrbracket} \otimes_{i \in\{1,2\}}\left(\operatorname{det}_{\mathbb{Z}_{p} \llbracket G_{\infty} \rrbracket} Z_{\infty}^{i}\left(\mathbb{Q}_{p}, T\right)\right)^{(-1)^{i+1}}
$$

et $\delta_{\text {st }}(V)=\prod_{j>-h} \ell_{-j}^{-\operatorname{dim}_{\mathbb{Q}_{p}} \operatorname{Fil}^{j} \mathbf{D}_{p}(V)} \delta_{h}\left(\Omega_{V}\right)\left(\operatorname{voir}[\mathbf{1 9}, 3.1 .5]\right.$, si $M$ est un $\mathcal{H}\left(G_{\infty}\right)$ module de torsion de type fini, on utilise l'application canonique

$$
\operatorname{det} M \longrightarrow \operatorname{Frac}\left(\mathcal{H}\left(G_{\infty}\right)\right) ;
$$

en fait tous les modules de torsion de type fini qui interviennent dans ce texte sont des $\mathbb{Q}_{p} \otimes \mathbb{Z}_{p} \llbracket G_{\infty} \rrbracket$-modules (ou $\mathbb{Z}_{p} \llbracket G_{\infty} \rrbracket$-modules) de type fini annulés par un élément non diviseur de 0 de $\mathbb{Z}_{p} \llbracket G_{\infty} \rrbracket$ et on a $\operatorname{det} M=\mathcal{H}\left(G_{\infty}\right) \operatorname{det}_{\mathbb{Q}_{p} \otimes \mathbb{Z}_{p} \llbracket G_{\infty} \rrbracket} M$ (ou $\left.\operatorname{det} \mathbb{Q}_{p} \otimes M=\mathcal{H}\left(G_{\infty}\right) \operatorname{det}_{\mathbb{Z}_{p} \llbracket G_{\infty} \rrbracket} M\right)$.

THÉORÈME B.2

1) $\delta_{\mathrm{st}}(V)=\mathcal{H}\left(G_{\infty}\right)$.

2) Soient $h$ et $h^{*} \geqslant 1$ tels que $\mathrm{Fil}^{-h} \mathbf{D}_{p}(V)=\mathbf{D}_{p}(V)$, Fil ${ }^{-h^{*}} \mathbf{D}_{p}\left(V^{*}(1)\right)=\mathbf{D}_{p}\left(V^{*}(1)\right)$. Si $x \in Z_{\infty}^{1}\left(\mathbb{Q}_{p}, V\right), \prod_{-h<j<h^{*}} \ell_{-j} x$ appartient à l'image de $\mathcal{D}_{\infty, f}\left(\mathbf{D}_{p}(V)\right)$ par $\Omega_{V, h}$.

Ainsi, l'application logarithme $\mathcal{L}_{V, h}$ inverse de $\Omega_{V, h}$ est un homomorphisme de $\mathbb{Z}_{p} \llbracket G_{\infty} \rrbracket$-modules

$$
\mathcal{L}_{V, h}: Z_{\infty}^{1}\left(\mathbb{Q}_{p}, V\right) \longrightarrow \xi_{h, h^{*}}^{-1} \mathcal{D}_{\infty, f}\left(\mathbf{D}_{p}(V)\right) \subset \xi_{h, h^{*}}^{-1} \mathcal{D}_{\infty}^{\text {gros }}\left(\mathbf{D}_{p}(V)\right)
$$

en posant $\xi_{h, h^{*}}=\prod_{-h<j<h^{*}} \ell_{-j}$.

4) On démontre avec ces outils le théorème suivant : 
ThÉORÈme C. - Soient $V$ une représentation p-adique semi-stable d'une extension finie non ramifiée $K$ de $\mathbb{Q}_{p}$ et $T$ un réseau de $V$ stable par $G_{K}$. Soit Fil ${ }^{1} V$ la plus grande sous-représentation de $V$ dont les poids de Hodge-Tate sont strictement positifs et $\mathrm{Fil}^{1} T=\mathrm{Fil}^{1} V \cap T$. Alors, la limite projective des $H_{g}^{1}\left(K\left(\mu_{p^{n}}\right), T\right)$ relativement aux applications de corestriction est de rang sur $\mathbb{Z}_{p} \llbracket G_{\infty} \rrbracket$ égal à $\left[K: \mathbb{Q}_{p}\right] \operatorname{dim} \mathrm{Fil}^{1} V$ et égale à torsion près à la limite projective des $H^{1}\left(K\left(\mu_{p^{n}}\right), \mathrm{Fil}^{1} T\right)$.

Bien que ce texte soit volontairement limité à la théorie locale, terminons cette introduction en évoquant l'application de ce texte au cas global (nous n'en parlerons plus ensuite, voir [27] dans le cas particulier des courbes elliptiques semi-stables). Soit $V$ une représentation $p$-adique géométrique de $G_{\mathbb{Q}}$, semi-stable en $p[\mathbf{1 1}]$ : ainsi $V$ est non ramifiée en en dehors d'un nombre fini de places $S$ (que l'on suppose contenir $p$ ) et semi-stable en $p$; soit $\overline{\mathbb{Q}}_{S}$ la plus grande extension de $\mathbb{Q}$ non ramifiée en dehors de $S: V$ est donc une représentation de $\operatorname{Gal}\left(\mathbb{Q}_{S} / \mathbb{Q}\right)$. Soit $T$ un réseau de $V$ stable par $\operatorname{Gal}\left(\mathbb{Q}_{S} / \mathbb{Q}\right)$. Comment faire la théorie des fonctions $L$-adiques de $V$ en utilisant la théorie faite ici? Ou plutôt comment construire le module arithmétique des fonctions $L$-adiques de $V$ en utilisant

$$
\mathcal{L}_{V, h}: Z_{\infty}^{1}\left(\mathbb{Q}_{p}, V\right) \longrightarrow \xi_{h, h^{*}}^{-1} \mathcal{D}_{\infty}^{\text {gros }}\left(\mathbf{D}_{p}(V)\right) ?
$$

Soit $H_{\infty, S}^{i}(\mathbb{Q}, T)=\lim _{n} H^{i}\left(\overline{\mathbb{Q}}_{S} / \mathbb{Q}\left(\mu_{p^{n}}\right), T\right)$ et $H_{\infty, S}^{i}(\mathbb{Q}, V)=\mathbb{Q}_{p} \otimes H_{\infty, S}^{i}(\mathbb{Q}, T)$. Les $\mathbb{Z}_{p} \llbracket G_{\infty} \rrbracket$-modules $H_{\infty, S}^{i}(\mathbb{Q}, T)$ sont de type fini. De plus, $H_{\infty, S}^{1}(\mathbb{Q}, T)$ ne dépend pas de $S$, nous le noterons $H_{\infty}^{1}(\mathbb{Q}, T)$. Il est conjecturé que $H_{\infty, S}^{2}(\mathbb{Q}, T)$ est de torsion. Cela implique que si $\delta$ est un caractère de $\Delta$ de parité $\epsilon(\delta)= \pm 1$, le $\delta$-espace propre $H_{\infty}^{1}(\mathbb{Q}, T)^{(\delta)}$ de $H_{\infty}^{1}(\mathbb{Q}, T)$ est de rang $d_{-\epsilon(\delta)}$ sur $\mathbb{Z}_{p} \llbracket \Gamma \rrbracket$ où $d_{ \pm}$est la dimension du sous-espace vectoriel de $V$ sur lequel la conjugaison complexe agit par \pm . Notons $\operatorname{det}_{\delta}$ (resp. $\wedge_{\delta}^{r}$ ) le déterminant (resp. la puissance $r$-ième extérieure) de l'espace propre associé à $\delta\left(\operatorname{sur} \mathbb{Z}_{p} \llbracket G_{\infty} \rrbracket\right.$ ou sur $\mathcal{H}\left(G_{\infty}\right)$ selon le contexte). Posons comme dans [21, $1.4]$ en rajoutant un indice $\delta$ pour indiquer que l'on a pris la $\delta$-composante

$$
\begin{aligned}
\Delta_{\infty, S, \delta}^{\text {glob }} & =\left(\operatorname{det}_{\delta} H_{\infty, S}^{2}(\mathbb{Q}, T)\right) \otimes\left(\operatorname{det}_{\delta} H_{\infty}^{1}(\mathbb{Q}, T)\right)^{-1} \\
Z_{\infty, S}^{i}(\mathbb{Q}, T) & =\varliminf_{n}^{\lim } \oplus_{v \in S} H^{i}\left(\mathbb{Q}\left(\mu_{p^{n}}\right)_{v}, T\right) .
\end{aligned}
$$

On déduit de $\mathcal{L}_{V, h}$ des homomorphismes de $\mathbb{Z}_{p} \llbracket \Gamma \rrbracket$-modules :

$$
L_{V, h, \delta}:\left(\Delta_{\infty, S, \delta}^{\text {glob }}\right)^{-1} \longrightarrow \wedge_{\delta}^{d_{-\epsilon(\delta)}} Z_{\infty, p}^{1}(\mathbb{Q}, T) \stackrel{\mathcal{L}_{V, h}}{\longrightarrow} \operatorname{Frac}(\mathcal{B}(\Gamma)) \wedge_{\delta}^{d_{-\epsilon(\delta)}} \mathcal{D}_{\infty}^{\text {gros }}\left(\mathbf{D}_{p}(V)\right)
$$

Fixons une base e $\operatorname{de} \operatorname{det}_{\delta} \mathcal{D}_{\infty}^{\text {gros }}\left(\mathbf{D}_{p}(V)\right)$. On peut alors définir le module arithmétique associé à $V$ et à $\mathbb{Q}\left(\mu_{p} \infty\right)$ (en reprenant les notations de [21]) comme la famille des sous- $\mathbb{Z}_{p} \llbracket G_{\infty} \rrbracket$-modules $\mathbf{I}_{\text {arith },\{p, \infty\}, h}(T)_{ \pm}(\omega)$ de $\operatorname{Frac}\left(\mathcal{B}\left(G_{\infty}\right)\right)$ définis pour 


$$
\begin{aligned}
& \omega \in \wedge_{\delta}^{d_{-\epsilon(\delta)}} \mathcal{D}_{\infty}^{\operatorname{gros}}\left(\mathbf{D}_{p}(V)\right) \text { par } \\
& \mathbf{I}_{\text {arith },\{p, \infty\}, h}(T)_{ \pm}(\omega) \mathbf{e} \\
& \quad \quad=\prod_{j>-h} \ell_{-j}^{-\operatorname{dim}_{\mathbb{Q}_{p}} \operatorname{Fil}^{j} \mathbf{D}_{p}(V)} \operatorname{det}_{\delta} Z_{\infty, S-\{p\}}^{2}(\mathbb{Q}, T) \cdot \omega \wedge L_{V, h, \delta}\left(\Delta_{\infty, S}^{\text {glob }}(T)^{-1}\right)
\end{aligned}
$$

pour $h$ assez grand. Lorsque $V$ est cristalline en $p$, on peut associer à toute base de $\mathbf{D}_{p}(V)$ une base de $\mathcal{D}_{\infty}^{\text {gros }}\left(\mathbf{D}_{p}(V)\right)$, que l'on peut considérer comme canonique, grâce à l'homomorphisme $d \mapsto d(1+x)$. Cela permet dans la définition (D) de remplacer $\omega$ par un élément de $\wedge^{d_{\epsilon(\delta)}} \mathbf{D}_{p}(V)$ et e par un élément de $\operatorname{det} \mathbf{D}_{p}(V)$. Ces éléments ont le grand avantage d'être stables par dérivation et donc par twist : $D(d(1+x))=d(1+x)$. Mais surtout, ils appartiennent au $\mathcal{H}\left(G_{\infty}\right)$-module $\mathcal{D}_{\infty, f}\left(\mathbf{D}_{p}(V)\right)$ qui est simplement ici $\mathcal{H}^{\psi=0} \otimes \mathbf{D}_{p}(V)$.

Lorsque $V$ est seulement semi-stable, on peut penser à associer à une base de $\mathbf{D}_{p}(V)$ une base de $\mathcal{D}_{\infty}^{\text {gros }}\left(\mathbf{D}_{p}(V)\right)$ par l'application

$$
\mathcal{E}: d \longmapsto \exp \left(-p^{-1} \varphi(\log x) \cdot N\right)(d)(1+x) \text {. }
$$

Mais les $\mathcal{E}(d)$ ne sont pas stables par dérivation et surtout n'appartiennent en général à aucun des $\mathcal{D}_{\infty, *}\left(\mathbf{D}_{p}(V)\right)$. Et bien sûr, les composantes de $\mathcal{L}_{V, h}$ ne sont pas alors a priori dans $\mathcal{H}\left(G_{\infty}\right)$ mais seulement dans $\mathcal{B}\left(G_{\infty}\right)$. Il est donc préférable de se donner une base de $\mathcal{D}_{\infty, f}\left(\mathbf{D}_{p}(V)\right)$ : en général (c'est-à-dire lorsque $\varphi$ n'admet de valeur propre égale à une puissance de $p$ ), pour $d$ parcourant une base de $\mathbf{D}_{p}(V)$, il existe $\mathcal{V}(d)$ dans $\mathcal{D}_{\infty, f}\left(\mathbf{D}_{p}(V)\right)$ congru à $d(1+x)$ modulo $\mathcal{B}[\log x] \otimes \Delta(d)$ avec $\Delta(d)$ le $(\varphi, N)$-module engendré par $d$. À défaut d'être aussi explicite que $1+x$, nous donnons dans le texte un procédé de construction. Si $\omega$ est construit à partir d'une telle base, il existe $f \in \mathbb{Z}_{p} \llbracket G_{\infty} \rrbracket$ (non diviseur de zéro et en général égal à 1 ) tel que

$$
f \mathbf{I}_{\text {arith },\{p, \infty\}, h}(T)_{ \pm}(\omega) \in \mathcal{H}\left(G_{\infty}\right) .
$$

Remarquons que $\mathcal{D}_{\infty, f}\left(\mathbf{D}_{p}(V)\right)$ ne dépend que de la structure de $(\varphi, N)$-modules de $\mathbf{D}_{p}(V)$ et ne dépend pas de la filtration. Seule, l'application régulateur en dépend.

Peut-être faut-il simplement retenir de la définition du module arithmétique des fonctions $L p$-adiques qu'il mesure la position de la cohomologie globale dans un $\mathcal{B}\left(G_{\infty}\right)$-module libre construit directement à partir de $\mathbf{D}_{p}(V)$, i.e. la position de

$$
\prod_{j>-h} \ell_{-j}^{-\operatorname{dim}_{Q_{p}} \mathrm{Fi}^{j} \mathbf{D}_{p}(V)} \operatorname{det}_{\delta} Z_{\infty, S-\{p\}}^{2}(\mathbb{Q}, T) L_{V, h, \delta}\left(\Delta_{\infty, S}^{\mathrm{glob}}(T)^{-1}\right)
$$

dans

$$
\operatorname{Frac}(\mathcal{B}(\Gamma)) \wedge_{\delta}^{d_{-\epsilon(\delta)}} \mathcal{D}_{\infty}^{\text {gros }}\left(\mathbf{D}_{p}(V)\right)
$$

Toutes les autres définitions consistent à prendre des bases et à calculer les coordonnées dans cette base.

Prenons le cas où $V$ est de dimension 2 sur $\mathbb{Q}_{p}$, semi-stable et non cristalline. Soit $e_{1}, e_{2}=N e_{1}$ une base de $\mathbf{D}_{p}(V)$ avec $e_{1}$ un vecteur propre de $\varphi: \varphi e_{1}=\alpha e_{1}$; 
on a alors $\varphi e_{2}=p^{-1} \alpha e_{2}, N e_{2}=0$. Reprenons les deux points de vue évoqués au paragraphe précédent. On a $\mathcal{E}\left(e_{2}\right)=(1+x) e_{2}$. Quant à $\mathcal{E}\left(e_{1}\right)$, il vaut

$$
\begin{aligned}
\mathcal{E}\left(e_{1}\right) & =(1+x) \cdot e_{1}-p^{-1} \varphi \log x(1+x) \cdot N e_{1} \\
& =(1+x) \cdot e_{1}-p^{-1} \varphi \log x(1+x) \cdot e_{2} .
\end{aligned}
$$

Si $\omega_{\text {glob }}$ est une base de $\left(\operatorname{det} H_{\infty,\{p\}}^{2}(\mathbb{Q}, T)\right) \otimes\left(\operatorname{det} H_{\infty}^{1}(\mathbb{Q}, T)\right)^{-1}$, avec $H_{\infty,\{p\}}^{2}(\mathbb{Q}, T)$ le noyau de $H_{\infty,\{p\}}^{2}(\mathbb{Q}, T) \rightarrow Z_{\infty, S-\{p\}}^{2}(\mathbb{Q}, T)$, on a

$$
\mathcal{L}_{V, 0}\left(\omega_{\text {glob }}\right) \in \mathcal{D}_{\infty}^{\text {gros }}(V) \cong \mathcal{B}\left(G_{\infty}\right) \mathcal{E}\left(e_{1}\right) \oplus \mathcal{B}\left(G_{\infty}\right) N e_{1}
$$

et peut donc s'écrire

$$
\begin{aligned}
& f_{1} \cdot(1+x)\left(e_{1}-p^{-1} \varphi(\log x) N e_{1}\right)+f_{2} \cdot(1+x) N e_{1} \\
& \quad=f_{1} \cdot(1+x) e_{1}+\left(f_{2} \cdot(1+x)-f_{1} \cdot\left(p^{-1} \varphi(\log x)(1+x)\right)\right) N e_{1}
\end{aligned}
$$

avec $f_{1}$ et $f_{2}$ dans $\mathcal{B}\left(G_{\infty}\right)$. En projetant sur $\mathbf{D}_{p}(V) / N \mathbf{D}_{p}(V)$, il est facile de vérifier que $f_{1}$ est dans $\mathcal{H}\left(G_{\infty}\right)$. Par contre, $f_{2}$ n'a pas de raison d'appartenir à $\mathcal{H}\left(G_{\infty}\right)$ lorsque $V$ n'est pas cristalline.

Si maintenant on fixe une base de $\mathcal{D}_{\infty, f}(D)$ avec $D$ le $(\varphi, N)$-module $\mathbf{D}_{p}(V)$ (en fait c'est une base d'un $\mathcal{H}\left(G_{\infty}\right)$-module qui est un peu plus gros que $\mathcal{D}_{\infty, f}(D)$ lorsque $\alpha$ est une puissance de $p): \mathcal{T}_{2}=(1+x) e_{2}$ et $\mathcal{T}_{1}=(1+x) e_{1}+t_{0} e_{2}$, on peut écrire

$$
\mathcal{L}_{V, 0}\left(\omega_{\text {glob }}\right)=L_{1} \cdot \mathcal{T}_{1}+L_{2} \cdot \mathcal{T}_{2}
$$

avec $L_{1}$ et $L_{2}$ appartenant à $\mathcal{H}\left(G_{\infty}\right)$. Il est clair que $L_{1}=f_{1}$.

Lorsque $V$ provient d'une forme modulaire $f$ pour $X_{0}(N)$ (dont le conducteur est alors exactement divisible par $p$ ), la conjecture principale classique prédit que le $\mathbb{Z}_{p} \llbracket G_{\infty} \rrbracket$-module $\mathcal{I}_{\text {arith }}$ engendré par $L_{1}$, c'est-à-dire la projection sur $e_{1}$ de

$$
\operatorname{det} H_{\infty,\{p\}}^{2}(\mathbb{Q}, T) \mathcal{L}_{V, 0}\left(H_{\infty}^{1}(\mathbb{Q}, T)\right)
$$

admet comme générateur la fonction $L$-adique $L_{p, \alpha}(f)$ construite par interpolation par Mazur-Tate-Teitelbaum (en ayant conjecturé auparavant que $H_{\infty}^{1}(\mathbb{Q}, T)$ est de rang 1). Le travail fondamental de Kato et sa construction d'éléments explicites et modulaires de $H_{\infty}^{1}(\mathbb{Q}, T)$ permettent de montrer que $L_{p, \alpha}(f)$ appartient à $\mathcal{I}_{\text {arith }}$. Plus précisément, Kato construit un élément $c_{\infty}$ de $H_{\infty}^{1}(\mathbb{Q}, T)$ tel que

$$
\mathcal{L}_{V, 0}\left(c_{\infty}\right)=L_{p, \alpha}(f) \cdot \mathcal{T}_{1}+L_{p, \beta}(f) \cdot \mathcal{T}_{2}
$$

(aux facteurs d'Euler aux places de mauvaise réduction près). Cela permet de voir qu'il existe d'autres fonctions de $\mathcal{H}\left(G_{\infty}\right)$ interpolant les valeurs spéciales des fonctions $L$ correspondant par la conjecture principale à $L_{2}$. Disons pour finir que lorsque $\alpha=p^{s}$, le fait que $L_{p, \alpha}(f)$ a un zéro en $\chi^{-s}$ vient de ce que, dans ce cas, ce n'est pas $\left(\mathcal{T}_{1}, \mathcal{T}_{2}\right)$ qui forme une base de $\mathcal{D}_{\infty, f}(D)$ mais $\left(\left(\chi(\tau)^{s} \tau-1\right) \mathcal{T}_{1}, \mathcal{T}_{2}\right)$ pour $\tau$ un générateur de $G_{\infty}$. Le calcul de la dérivée de $L_{p, \alpha}(f)$ en $\chi^{-s}$ est alors très simple et l'apparition du paramètre associé à la filtration de $\mathbf{D}_{p}(V)$ très naturel. Nous renvoyons à [27]. 
Donnons un résumé de chacun des paragraphes. Le premier paragraphe est consacré à l'étude de certains anneaux de fonctions, par ailleurs bien connus, et à leur structure de $G_{\infty}$-modules. Dans les $\S 2, \S 3, \S 4$, nous faisons l'étude des $G_{\infty}$-modules associés à un $(\varphi, N)$-module, calculons leur rang et montrons les suites exactes qui les relient. Pour cela (§3), nous donnons une méthode pour construire des éléments. Ces trois paragraphes peuvent être réduits à un strict minimum si on se limite au cas où $\varphi$ n'a pas de valeur propre égale à une puissance de $p$. Dans le cinquième paragraphe, nous introduisons la cohomologie galoisienne et construisons l'exponentielle. Puis nous déduisons des lois de réciprocité de Colmez le calcul du déterminant. Dans le sixième paragraphe, nous expliquons ce qui doit être changé à $[\mathbf{2 6}]$ pour calculer le rang des normes universelles.

Quelques calculs plus ou moins classiques dus en grande partie à Coleman sur le polylogarithme ont leur place comme illustration. Nous les avons regroupés dans l'appendice A tout en les signalant dans le texte principal. Dans l'appendice B, nous reprenons la démonstration de Cherbonnier-Colmez qui permet de calculer la structure de $G_{\infty}$-modules de $\mathcal{B}^{\psi=0}$.

Notations : Si $M$ est un $G_{K}$-module (éventuellement trivial), $M(k)$ désigne le $k$ ième twist cyclotomique de $M$ : l'action de Galois est modifiée par $\chi^{k}$. Si $M$ est un $\varphi$-module, $M[k]$ désigne le $\varphi$-module décalé $k$-fois : le Frobenius est multiplié par $p^{k}$. On désigne par $\zeta_{n}$ un système compatible de racines de l'unité d'ordre $p^{n}: \zeta_{n+1}^{p}=\zeta_{n}$.

Je tiens à remercier le rapporteur pour ses remarques qui m'ont obligée à préciser certains points. 


\section{CHAPITRE 1}

\section{ANNEAUX DE FONCTIONS}

\subsection{L'anneau $\mathcal{B}$}

1.1.1. Si $I$ est un intervalle de $\mathbb{R}^{+}$, on définit les espaces de fonctions analytiques :

$$
\mathcal{H}^{I}=\left\{\sum_{n \in \mathbb{Z}} a_{n} x^{n} \text { t.q. } a_{n} \in \mathbb{Q}_{p} \text { et } \lim _{n \rightarrow \pm \infty}\left|a_{n}\right| r^{n}=0 \text { pour tout } r \in I\right\}
$$

Par exemple, si $I$ est fermé et égal à $\left[r_{1}, r_{2}\right]$ avec $0<r_{1} \leqslant r_{2}$, on a

$$
\mathcal{H}^{I}=\left\{\sum_{n \in \mathbb{Z}} a_{n} x^{n} \text { t.q. } a_{n} \in \mathbb{Q}_{p} \text { et }\left\{\begin{array}{l}
\lim _{n \rightarrow-\infty}\left|a_{n}\right| r_{1}^{n}=0 \\
\lim _{n \rightarrow+\infty}\left|a_{n}\right| r_{2}^{n}=0
\end{array}\right\}\right. \text {. }
$$

Pour $\rho \in I$, on $\operatorname{asup}_{|x|=\rho}|f(x)|=\sup _{n}\left|a_{n}\right| \rho^{n}$ et la valeur commune est notée $\|f\|_{\rho}$. On définit

$$
\mathcal{H}^{\left[r_{1}, r_{2}\right)}=\left\{\sum_{n \in \mathbb{Z}} a_{n} x^{n} \in \mathcal{H}^{\left[r_{1}, r_{2}[\right.} \text { tel que } \sup \left|a_{n}\right| r_{2}^{n}<\infty\right\}
$$

En particulier,

$$
\mathcal{H}^{[r, 1)}=\left\{\sum_{n \in \mathbb{Z}} a_{n} x^{n} \text { tel que } a_{n} \in \mathbb{Q}_{p} \text { et }\left\{\begin{array}{l}
\lim _{n \rightarrow-\infty}\left|a_{n}\right| r^{n}=0 \\
\sup \left|a_{n}\right|<\infty
\end{array}\right\}\right.
$$

On définit pour $I$ de la forme $\left[r_{1}, r_{2}\right\}$ avec $\left.\}=\right]$ ou ),

$$
\mathcal{H}_{0}^{I}=\left\{\sum_{n \in \mathbb{Z}} a_{n} x^{n} \in \mathcal{H}^{I} \text { tel que } \sup _{n \in \mathbb{Z}}\left(\left|a_{n}\right| r_{1}^{n},\left|a_{n}\right| r_{2}^{n}\right) \leqslant 1\right\}
$$

On a donc $\mathcal{H}^{I}=\mathcal{H}_{0}^{I}[1 / p]$. Par le principe du maximum, pour tout $\rho \in I,\|f\|_{\rho} \leqslant 1$ pour $f \in \mathcal{H}_{0}^{I}$. On munit $\mathcal{H}^{I}$ de la topologie de convergence uniforme sur les $|x| \in J$ pour $J$ sous-ensemble fermé de $I$ ( $\left[r_{1}, r_{2}\right)$ étant considéré comme ouvert en $\left.r_{2}\right)$. Les espaces $\mathcal{H}^{I}$ sont complets. On pose pour $f \in \mathcal{H}^{I}$ avec $I=\left[r_{1}, r_{2}\right]$,

$$
\|f\|_{\mathrm{sp}}=\|f\|_{\mathrm{sp}, I}=\sup _{n \in \mathbb{Z}}\left(\left|a_{n}\right| r_{1}^{n},\left|a_{n}\right| r_{2}^{n}\right) .
$$


Ainsi, $\mathcal{H}_{0}^{I}$ est le sous- $\mathbb{Z}_{p}$-module des éléments de $\mathcal{H}^{I}$ de norme $\leqslant 1$. Une suite $f_{n}$ d'éléments de $\mathcal{H}^{I}$ converge vers 0 dans $\mathcal{H}^{I}$ si et seulement si pour tout $\rho \in I,\left\|f_{n}\right\|_{\rho}$ tend vers 0 . On note $\mathcal{B}^{\prime}$ la réunion des $\mathcal{H}^{[\rho, 1[}$ pour $\rho \in[0,1[$. Nous travaillerons en fait essentiellement dans $\mathcal{H}^{[\rho, 1[}$ avec $\rho$ fixé.

1.1.2. Pour tout $h>0$, soit $\mathcal{B}_{h}$ le $\mathbb{Q}_{p}$-espace vectoriel des éléments $f$ de $\mathcal{B}^{\prime}$ tels que $\lim _{n \rightarrow \infty} p^{-h n}\|f\|_{\rho_{n}}=0$ pour $\rho_{n}=\rho^{1 / p^{n}}$ pour un $\rho<1$ (ou pour tout $\rho<1$, ce qui revient au même, on prendra en général dans la suite $\rho_{0}=p^{-1 /(p-1)}$, $\left.\rho_{n}=p^{-1 /\left((p-1) p^{n}\right)}\right)$. On note $\mathcal{B}$ la sous-algèbre de $\mathcal{B}^{\prime}$, réunion des $\mathcal{B}_{h}$. Si $f \in \mathcal{B}$, on note $\mathfrak{o}(f)$ la borne inférieure des $h \in \mathbb{R}^{+}$tels que $f \in \mathcal{B}_{h}$; on l'appelle l'ordre de $f$. On pose aussi $\mathcal{H}=\mathcal{B} \cap \mathcal{H}^{[0,1[}$.

L'intersection de $\mathcal{B}^{\prime}$ avec $\mathbb{Q}_{p} \llbracket x \rrbracket$ est l'espace des fonctions analytiques sur la boule $B\left(0,1^{-}\right)=\left\{x \in \mathbb{C}_{p},|x|<1\right\}$. L'intersection de $\mathcal{B}^{\prime}$ avec $\mathbb{Q}_{p} \llbracket 1 / x \rrbracket$ est l'espace des fonctions analytiques sur le complémentaire d'une boule

$$
B\left(0, \rho^{-}\right)=\left\{x \in \mathbb{C}_{p} \text { tel que }|x|<\rho\right\}
$$

avec $\rho<1$. Plus précisément, $\mathcal{H}^{[\rho, 1)} \cap \mathbb{Q}_{p} \llbracket 1 / x \rrbracket=\mathcal{H}^{[\rho, \infty)}$. On notera avec un indice + (resp. - ) l'intersection d'un sous-anneau de $\mathcal{B}$ avec $\mathbb{Q}_{p} \llbracket x \rrbracket$ (resp. $\left.\mathbb{Q}_{p} \llbracket 1 / x \rrbracket\right)$. Avec les notations des articles précédents, $\mathcal{H}=\mathcal{B}_{+}=\mathcal{B} \cap \mathbb{Q}_{p} \llbracket x \rrbracket$.

1.1.3. Le corps des fractions de $\mathbb{Z}_{p} \llbracket x \rrbracket$ s'injecte dans $\mathcal{B}$. En effet, par le théorème de préparation de Weierstrass, il suffit de vérifier que tout polynôme unitaire de $\mathbb{Z}_{p}[x]$ est inversible dans $\mathcal{B}$. En passant à $\mathbb{C}_{p}$, il suffit de montrer que cela est le cas pour les polynômes de degré 1 . Lorsque $|r| \geqslant 1$, on écrit

$$
(x-r)^{-1}=-r^{-1}\left(1-\frac{x}{r}\right)^{-1}=-r^{-1} \sum_{n=0}^{\infty} \frac{x^{n}}{r^{n}} \in \mathbb{C}_{p} \otimes \mathcal{O}_{\mathbb{C}_{p}} \llbracket x \rrbracket
$$

$\left(\mathcal{O}_{\mathbb{C}_{p}}\right.$ étant l'anneau des entiers de $\left.\mathbb{C}_{p}\right)$. Lorsque $|r|<1$,

$$
(x-r)^{-1}=x^{-1}\left(1-\frac{r}{x}\right)^{-1}=x^{-1} \sum_{n=0}^{\infty} \frac{r^{n}}{x^{n}} .
$$

et cette série de Laurent converge pour $|x|>|r|$ et appartient à $\mathcal{O}_{\mathbb{C}_{p}} \otimes \mathcal{B}$ puisque $|r|<1$. Il est facile de redescendre à $\mathbb{Q}_{p}$.

1.1.4. Le groupe $G_{\infty}=\operatorname{Gal}\left(\mathbb{Q}_{p}\left(\mu_{p^{\infty}}\right) / \mathbb{Q}_{p}\right)$ agit naturellement sur les $\mathcal{H}^{[\rho, 1[}$ par prolongement continu de l'action de $G_{\infty}$ donnée par $\tau(x)=(1+x)^{\chi(\tau)}-1$ et

$$
\tau(1 / x)=\frac{1}{(1+x)^{\chi(\tau)}-1}=\frac{\chi(\tau)^{-1}}{x}\left(1+x Q_{\tau}(x)\right)^{-1}
$$

où $Q_{\tau}(x) \in \mathbb{Z}_{p} \llbracket x \rrbracket$ et agit donc sur $\mathcal{B}^{\prime}$. Remarquons que $G_{\infty}$ conserve $\mathcal{B}, \mathcal{H}=\mathcal{B}_{+}$mais ne conserve pas $\mathcal{B}_{-}$. 
1.1.5. Soit $\varphi(x)=(1+x)^{p}-1 \in x \mathbb{Z}_{p} \llbracket x \rrbracket$ et $\varphi(1 / x)$ le développement de $1 /\left((1+x)^{p}-1\right)$ en $1 / x$

$$
\begin{aligned}
\varphi(1 / x) & =\frac{1}{x^{p}\left((1+1 / x)^{p}-1 / x^{p}\right)}=\frac{1}{x^{p}(1+p(1 / x) Q(1 / x))} \\
& =\frac{1}{x^{p}} \sum_{n \geqslant 0}\left(\frac{p}{x} Q\left(\frac{1}{x}\right)\right)^{n} \in\left(\frac{1}{x^{p}}\right) \mathbb{Z}_{p} \llbracket \frac{1}{x} \rrbracket
\end{aligned}
$$

avec $Q$ un polynôme à coefficients entiers. Pour $|x|>p^{-1 /(p-1)},|\varphi(x)|=|x|^{p}$ et $|\varphi(1 / x)|=|1 / x|^{p}$; si $|x|<p^{-1 /(p-1)}$, on a $|\varphi(x)|=|x| / p$; si $|x|=p^{-1 /(p-1)}$, on a $|\varphi(x)| \leqslant|x| / p=\left|x^{p}\right|$.

On définit un opérateur $\varphi$ de l'algèbre $\mathcal{B}^{\prime}$ prolongeant ces définitions :

$$
\varphi\left(\sum_{n \in \mathbb{Z}} a_{n} x^{n}\right)=\sum_{n \geqslant 0} a_{n} \varphi(x)^{n}+\sum_{n<0} a_{n} \varphi(1 / x)^{n} .
$$

L'opérateur $\varphi$ conserve les parties + et - de $\mathcal{B}$. Si $|x|>p^{-1 /(p-1)}$, on a $|\varphi(x)|=|x|^{p}$. Le principe du maximum et le fait que pour $|y|>p^{-p /(p-1)}$ l'équation $\varphi(x)=y$ a une solution en $x$ avec $|x|>p^{-1 /(p-1)}$ impliquent que

$$
\|\varphi(f)\|_{\rho}=\|f\|_{\rho^{p}} \text { si } \rho>p^{-1 /(p-1)}
$$

En particulier,

$$
\varphi\left(\mathcal{H}^{[\rho, 1)}\right) \subset \mathcal{H}^{\left[\rho^{1 / p}, 1\right)} \quad \text { si } \rho>p^{-p /(p-1)}=p^{-1 /(p-1)} / p .
$$

On peut alors définir un unique opérateur $\psi \mathrm{du} \mathbb{Q}_{p}$-espace vectoriel $\mathcal{B}^{\prime}$ tel que

$$
\varphi \circ \psi(f)=\frac{1}{p} \sum_{\zeta \in \mu_{p}} f(\zeta(1+x)-1)
$$

de la manière suivante. En appliquant cette équation fonctionnelle à $f=\varphi(g)$, on obtient que $\psi \circ \varphi$ est l'identité. En prenant $f=x^{k}$, l'équation fonctionnelle détermine de manière unique $\psi\left(x^{k}\right)$ et on a alors pour $\rho>p^{-1 /(p-1)}$,

$$
\left\|\psi\left(x^{k}\right)\right\|_{\rho}=\left\|\varphi \circ \psi\left(x^{k}\right)\right\|_{\rho^{1 / p}} \leqslant p\left\|x^{k}\right\|_{\rho^{1 / p}}
$$

car $|\zeta(1+x)-1|=|x|$ pour $|x|>p^{-1 /(p-1)}$. Pour $k<0, \psi\left(1 / x^{k}\right)$ est de la forme $Q_{k}(x) / x^{k}$ où $Q_{k}$ est un polynôme à coefficients dans $\mathbb{Z}_{p}$ de degré $<k$. En effet, on a

$$
\varphi \circ \psi\left(1 / x^{k}\right)=\frac{1}{p} \sum_{\zeta^{p}=1} \frac{1}{(\zeta(1+x)-1)^{k}}=\frac{P_{k}}{\varphi(x)^{k}}
$$

avec $\operatorname{deg} P_{k} \leqslant(p-1) k$. Le polynôme $P_{k}$ est nécessairement de la forme $Q_{k}(\varphi(x))$; le degré de $Q_{k}$ est inférieur à $k$. Le polynôme $P_{k}$ est à coefficients dans $\mathbb{Z} \llbracket x \rrbracket:$ il est en effet à coefficients dans $p^{-1} \mathbb{Z}$ et la réduction de $p P_{k}$ dans le corps des fractions de $\mathbb{Z} / p \mathbb{Z}((x))$ est

$$
\sum_{\zeta \in \mu_{p}}(\zeta(1+x)-1)^{-k} \equiv \sum_{\zeta \in \mu_{p}} x^{-k} \equiv 0 \quad \bmod p
$$


Donc $\psi\left(1 / x^{k}\right)$ appartient à l'intersection de $\mathbb{Z}_{p} \llbracket 1 / x \rrbracket$ et de $\left(1 / x^{k}\right) \mathbb{Z}_{p}[x]$ et

$$
\left\|\psi\left(1 / x^{k}\right)\right\|_{\rho} \leqslant\left\|1 / x^{k}\right\|_{\rho}=\rho^{-k} .
$$

Il est facile de déduire de ces propriétés que l'image par $\psi$ d'un élément de $\mathcal{B}^{\prime}$ (resp. $\mathcal{B}$ ) est dans $\mathcal{B}^{\prime}$ (resp. $\left.\mathcal{B}\right)$, que $\psi$ conserve les parties + et - de $\mathcal{B}$, que pour $\rho>p^{-1 /(p-1)}$ et $\}=[$, ] ou $)$, l'image de $\mathcal{H}^{\left[\rho^{p}, 1\right\}}$ par $\psi$ est contenue dans $\mathcal{H}^{\left[\rho^{p}, 1\right\}}$ et que $\psi\left(\mathcal{H}_{0}^{[\rho, 1\}}\right)$ est contenu dans $\mathcal{H}_{0}^{[\rho, 1\}}$.

1.1.6. LEMME

1) Soit $g \in \mathcal{B}$. Alors, pour $\rho \gg 0$

$$
\|\psi(g)\|_{\rho} \leqslant p\|g\|_{\rho^{1 / p}} .
$$

Si $g \in \mathcal{H}^{[\rho, \infty)}$, on $a$

$$
\|\psi(g)\|_{\rho} \leqslant\|g\|_{\rho} .
$$

2) Soit $u \in \mathcal{H}_{0}^{[\rho, 1)}$ et $g \in \mathcal{H}^{[\rho, 1[}$ avec $\rho \gg 0$,

$$
\|u g\|_{\rho} \leqslant\|g\|_{\rho} .
$$

Ici (et ailleurs), $\rho \gg 0$ signifie que $\rho$ est supérieur à $p^{-1 /(p-1)}$ et assez grand pour que $g \in \mathcal{H}^{[\rho, 1[}$.

Démonstration. - Les deux premières inégalités se déduisent des inégalités correspondantes pour $x^{k}$. La partie 2 est immédiate car $\|u\|_{\rho} \leqslant \sup \left(\|u\|_{1},\|u\|_{\rho}\right) \leqslant 1$.

Donnons quelques formules sur $\psi$ :

$$
\begin{aligned}
\psi(f \varphi(g)) & =\psi(f) g, \\
\psi(1+x) & =0, \\
\psi\left(\log ^{k}(1+x)\right) & =p^{-k} \log ^{k}(1+x) .
\end{aligned}
$$

Si $\lambda$ est un élément de $\mathbb{Z}_{p}[\Delta] \otimes \mathcal{H}(\Gamma): \lambda=\sum_{\delta \in \Delta} \delta \otimes g_{\delta}(\gamma-1)$ avec $g_{\delta} \in \mathcal{H}$ et $\gamma$ générateur topologique de $\Gamma$, on pose pour $\rho<1,\|\lambda\|_{\rho}=\sup _{\delta \in \Delta}\left\|g_{\delta}\right\|_{\rho}$ et $\mathfrak{o}(\lambda)=\sup _{\delta \in \Delta} \mathfrak{o}\left(g_{\delta}\right)$.

1.1.7. Proposition. - L'anneau $\mathcal{B}$ est muni d'une structure de $\mathcal{H}\left(G_{\infty}\right)$-module prolongeant l'action de $G_{\infty}$ par continuité. Pour $\lambda \in \mathcal{H}(\Gamma)$ et $f \in \mathcal{B}$, on a pour $\rho \gg 0$,

$$
\|\lambda \cdot f\|_{\rho} \leqslant\|\lambda\|_{\rho^{p-1}}\|f\|_{\rho} \leqslant\|\lambda\|_{\rho}\|f\|_{\rho}
$$

et $\mathfrak{o}(\lambda \cdot f) \leqslant \mathfrak{o}(\lambda)+\mathfrak{o}(f)$.

Démonstration. - Commençons par vérifier que pour $\chi(\gamma)-1$ de valuation $p$-adique $m \geqslant 1$ et pour $f \in \mathcal{H}^{[\eta, 1[}$,

$$
\left\|(\gamma-1)^{i} \cdot f\right\|_{\rho} \leqslant\|f\|_{\rho} \rho^{i\left(p^{m}-1\right)} .
$$


Utilisons pour cela le lemme B.2.2 qui implique que pour $|x|=\rho>\sup \left(\rho_{m-1}, \eta\right)$ et $k \in \mathbb{Z},\left\|(\gamma-1) \cdot x^{k}\right\| \leqslant \rho^{k} \rho^{p^{m}-1}$. On en déduit que

$$
\|(\gamma-1) \cdot f\|_{\rho} \leqslant\|f\|_{\rho} \rho^{p^{m}-1} \text {. }
$$

En recommençant, on obtient l'inégalité (1.1.1). Si $\lambda=\sum_{i=0}^{\infty} a_{i}(\gamma-1)^{i}$ est un élément de $\mathcal{H}(\Gamma)$, la série $\sum_{i=0}^{\infty} a_{i}(\gamma-1)^{i} \cdot f$ est convergente dans $\mathcal{B}^{\prime}$ et on a

$$
\|\lambda \cdot f\|_{\rho} \leqslant\|\lambda\|_{\rho^{p-1}}\|f\|_{\rho} .
$$

Si maintenant $f \in \mathcal{B}$, il existe un réel $h>0$ tel que $p^{-n h}\|f\|_{\rho^{1 / p^{n}}}$ tend vers 0 lorsque $n$ tend vers l'infini; si $r$ est un réel positif tel que $p^{-n r}\|\lambda\|_{\rho^{1 / p^{n}}}$ tend vers 0 ,

$$
p^{-n(h+r)}\|\lambda \cdot f\|_{\rho^{1 / p^{n}}} \leqslant p^{-n r}\|\lambda\|_{\rho^{\left(p^{m}-1\right) / p^{n}}} p^{-n h}\|f\|_{\rho^{1 / p^{n}}}
$$

et tend vers 0 lorsque $n \rightarrow \infty$. Donc $\lambda \cdot f \in \mathcal{B}$.

1.1.8. ThÉORÈmE. - $\mathcal{B}^{\psi=0}$ est muni d'une action de $\mathcal{B}\left(G_{\infty}\right)$-module prolongeant par continuité l'action de $G_{\infty}$. De plus, il existe une unique application $\mathbb{Q}_{p}$-linéaire

$$
\mathcal{B}\left(G_{\infty}\right) \longrightarrow \mathcal{B}^{\psi=0}
$$

prolongeant $\lambda \mapsto \lambda \cdot(1+x)$ pour $\lambda \in \mathcal{H}\left(G_{\infty}\right)$ et c'est un isomorphisme de $G_{\infty}$-modules ; l'image de $\mathcal{H}\left(G_{\infty}\right)$ est $\mathcal{H}^{\psi=0}$.

En particulier, si $\tau \in G_{\infty}$ n'est pas de torsion, $\tau-1$ est bijectif sur $\mathcal{B}^{\psi=0}$. Ce théorème est essentiellement montré dans [3] ; aussi, renvoyons-nous la démonstration à l'appendice $\mathrm{B}$.

Par contre, il ne semble pas possible de prolonger cette action à $\mathcal{B}$.

1.1.9. Si $f$ est un élément de $\mathcal{B}\left(G_{\infty}\right)$, il existe un entier $n(f)$ tel que pour tout caractère $\eta$ de $G_{\infty}$ de conducteur $p^{n}$ avec $n \geqslant n(f)$ et tout entier $k \in \mathbb{Z}$, on puisse calculer $\eta \chi^{k}(f)$. L'entier $n(f)$ est déterminé par le fait que $f \in \mathcal{H}^{\left[\rho_{n(f)}, 1[\right.}$ et il s'agit de remarquer que si $u \in 1+p \mathbb{Z}_{p}$ et si $\zeta$ est une racine de l'unité d'ordre $p^{n}$, on a

$$
|\zeta u-1| \leqslant \sup (|u-1|,|\zeta-1|)=\sup \left(p^{-r}, p^{-1 /(p-1) p^{n-1}}\right)
$$

avec égalité si les deux nombres sont différents. En particulier, si $n \geqslant 1$, on a $|\zeta u-1|=\rho_{n}$ et $f$ peut être évalué sur $\eta \chi^{k}$ si $\eta$ est de conducteur $p^{n}$ avec $n \geqslant n(f)$.

\subsection{L'anneau $\mathcal{B}[\log x]$.}

1.2.1. Soit $\log x$ une branche du logarithme, c'est-à-dire n'importe quelle fonction localement analytique sur $\mathbb{C}_{p}-\{0\}$ vérifiant $\log x y=\log x+\log y$ et dont la dérivée en 1 est 1 . Vérifions que $\log x$ n'est pas algébrique sur le corps des fractions des fonctions analytiques sur une couronne $C=\left\{r_{1} \leqslant|x| \leqslant r_{2}\right\}$. En effet, soit $\log ^{k} x+\sum_{j=0}^{k-1} f_{j} \log ^{j} x=0$ une équation non triviale de degré minimal avec les $f_{j}$ des fonctions méromorphes sur la couronne $C$ (quotient de fonctions analytiques). En 
dérivant, on obtiendrait une équation de degré $k-1$ non identiquement nulle car $1 / x$ n'est pas la dérivée d'une fonction méromorphe sur $C^{(1)}$.

En particulier, l'anneau $\mathcal{B}[\log x]$ des polynômes en $\log x$ à coefficients dans $\mathcal{B}$ est une sous- $\mathbb{Q}_{p^{-}}$-algèbre de l'anneau $\mathcal{L} \mathcal{A}\left(B\left(0,1^{-}\right)-\{0\}\right)$ des fonctions localement analytiques sur $B\left(0,1^{-}\right)-\{0\}$ et $\mathcal{B}_{-}[\log x]$ est une sous- $\mathbb{Q}_{p}$-algèbre de l'anneau des fonctions localement analytiques sur $\mathbb{C}_{p}-\{0\}$. Si $h \in \mathcal{B}[\log x]$, on appelle degré de $h$ le degré de $h$ en tant que polynôme en $\log x$. Si $f \in \mathcal{B}[\log x]$ vérifie $f(x)=0$ pour $r_{1} \leqslant|x| \leqslant r_{2}<1$, $f$ est identiquement nul.

1.2.2. Soit $\eta>\rho_{0}$. Si $H=\sum_{k} H_{k} \log ^{k} x \in \mathcal{H}^{[\eta, 1}[[\log x]$, on pose pour $\eta<\rho<1$,

$$
\|H\|_{\rho}=\sup \left\|H_{k}\right\|_{\rho} .
$$

On munit ainsi $\mathcal{B}[\log x]$ de la convergence coefficient par coefficient dans $\mathcal{B}$ (en tant que polynômes en $\log x)$. Pour $\rho \gg 0, \mathcal{H}^{[\rho, 1[}[\log x]$ est complet.

1.2.3. Si $\tau \in G_{\infty}$, soit

$$
a_{\tau}=\log \frac{(1+x)^{\chi(\tau)}-1}{x} .
$$

Comme $\chi(\tau)$ est une unité $p$-adique, $a_{\tau}$ est un élément de $\mathcal{H}$ et plus précisément de $\mathcal{H}_{1}$. On pose pour $\tau \in G_{\infty}$

$$
\tau(\log x)=\log x+a_{\tau} .
$$

On en déduit une action continue de $G_{\infty} \operatorname{sur} \mathcal{B}[\log x]$ prolongeant l'action sur $\mathcal{B}$. Remarquons que l'action de $G_{\infty}$ ne conserve pas $\mathcal{B}_{-}[\log x]$.

1.2.4. On a

$$
\begin{aligned}
\log \frac{(1+x)^{p}-1}{x^{p}} & =\log \frac{\varphi(x)}{x^{p}}=\log \frac{p+\cdots+x^{p}}{x^{p}} \\
& =\log \left(1+\frac{p}{x}+\cdots+\frac{p}{x^{p-1}}\right)
\end{aligned}
$$

et $\log \left(1+p / x+\cdots+p / x^{p-1}\right)$ définit, en utilisant le développement de log en série entière $\log (1+z)=\sum_{n>0}(-1)^{n} z^{n} / n$, un élément $p a_{\varphi}$ de $\mathcal{H}^{\left.] \rho_{0}, \infty\right)}$ avec $\rho_{0}=p^{-1 /(p-1)}$. Plus précisément, $p a_{\varphi}$ appartient à $\mathcal{H}_{0}^{\left[\rho_{1}, \infty\right)}$ avec $\rho_{1}=\rho_{0}^{1 / p}$. Calculons $\left\|p a_{\varphi}\right\|_{\rho}$ pour $\rho \geqslant \rho_{1}$. On a

$$
\left\|p a_{\varphi}\right\|_{\rho} \leqslant\|\log (1+p y)\|_{\rho^{-(p-1)}}=\|\log (1+y)\|_{p^{-1} \rho^{-(p-1)}} .
$$

\footnotetext{
(1) On ne peut avoir $f^{\prime}=1 / x$ avec $f$ analytique $\operatorname{sur} C$, car cela signifierait que $\sum_{n=-\infty}^{+\infty} n a_{n} x^{n}=1$ ce qui est impossible; si maintenant $f$ est le quotient de deux fonctions analytiques sans l'être : $f=g / h$, nécessairement $h$ a un zéro $\alpha$ sur la couronne $C$ tel que $f(\alpha) \neq 0$, sinon $f$ serait analytique (voir lemme B.1.1 de l'appendice B) et il est facile de voir en regardant l'ordre de ce zéro que l'équation $h^{2}=x\left(g^{\prime} h-g h^{\prime}\right)$ est impossible.
} 
Comme $\rho \geqslant \rho_{1}$, on a $p^{-1} \rho^{-(p-1)} \leqslant p^{-1} p^{1 / p}=p^{-(p-1) / p} \leqslant \rho_{0}$. Or

$$
\|\log (1+x)\|_{\rho_{0}}=\sup _{n \geqslant 1}\left(\frac{p^{-n / p-1}}{|n|}\right)=\sup _{k \geqslant 0}\left(p^{k-\left(p^{k} /(p-1)\right.}\right)=p^{-1 /(p-1)}
$$

Ainsi, pour $\rho \geqslant \rho_{1},\left\|p a_{\varphi}\right\|_{\rho}$ est inférieur à 1 . Donc $p a_{\varphi}$ appartient à $\mathcal{H}_{0}^{[\rho, \infty)}$. On pose

$$
\varphi \log x=p\left(\log x+a_{\varphi}\right),
$$

ce qui permet de définir sur $\mathcal{B}[\log x]$ une action continue de $\varphi$ prolongeant l'action naturelle sur $\mathcal{B}$. On prolonge alors l'opérateur $\psi \operatorname{sur} \mathcal{B}[\log x]$ par

$$
\psi\left(\log ^{k} x\right)=\sum_{j=0}^{k}(-1)^{k-j}\left(\begin{array}{l}
k \\
j
\end{array}\right) p^{-j} \psi\left(a_{\varphi}^{k-j}\right) \log ^{j} x
$$

(équation obtenue en écrivant

$$
\begin{aligned}
\log ^{k} x & =\left(p^{-1} \varphi \log x-a_{\varphi}\right)^{k} \\
& =\sum_{j=0}^{k}(-1)^{k-j}\left(\begin{array}{l}
k \\
j
\end{array}\right) p^{-j} a_{\varphi}^{k-j} \varphi \log ^{j} x
\end{aligned}
$$

et en utilisant la propriété désirée de $\psi: \psi(f \varphi(g))=\psi(f) g)$. La formule

$$
\varphi \circ \psi(g)(x)=p^{-1} \sum_{\zeta \in \mu_{p}} g(\zeta(1+x)-1)
$$

se généralise à $\mathcal{B}[\log x]$. On a $\psi(\log x)=p^{-1} \log x$ et $\psi\left(a_{\varphi}\right)=0$. L'élément $a_{\varphi}$ que l'on note aussi $\mathfrak{L}_{1}^{(p)}$ est très relié à la fonction de Kubota-Leopoldt (voir l'appendice $\mathrm{A}$ ).

\subsubsection{LEMME}

1) Soit $h$ un élément de $\mathcal{H}_{0}^{[\rho, \infty)}[\log x]$ de degré $f$ en $\log x$. Alors, pour tout entier $n \geqslant 0, \psi^{n}(h) \in p^{-f n} \mathcal{H}_{0}^{[\rho, \infty)}[\log x]$. Ainsi, si $h \in \mathcal{B}_{-}[\log x]$, on a pour $\rho \gg 0$

$$
\|\psi(h)\|_{\rho} \leqslant p^{f}\|h\|_{\rho} .
$$

2) Soit $h$ un élément de $\mathcal{B}[\log x]$ de degré $f$ en $\log x$. Alors, pour $0 \ll \rho<1$,

$$
\|\psi(h)\|_{\rho} \leqslant p^{f+1}\|h\|_{\rho^{1 / p}} .
$$

Démonstration. - Comme $\log x=p^{-1} \varphi \log x-a_{\varphi}$,

$$
\log ^{k} x=\sum_{j=0}^{k} p^{-j}(-1)^{k-j}\left(\begin{array}{l}
k \\
j
\end{array}\right) a_{\varphi}^{k-j} \varphi \log ^{j} x
$$

donc pour $g \in \mathcal{B}$,

$$
\psi\left(g \log ^{k} x\right)=\sum_{j=0}^{k}(-1)^{k-j} p^{-j}\left(\begin{array}{l}
k \\
j
\end{array}\right) \psi\left(g a_{\varphi}^{k-j}\right) \log ^{j} x .
$$


Pour $h=\sum_{k=0}^{f} h_{k} \log ^{k} x$, on a

$$
\begin{aligned}
\psi(h) & =\sum_{k=0}^{f} \sum_{j=0}^{k}(-1)^{k-j} p^{-j}\left(\begin{array}{l}
k \\
j
\end{array}\right) \psi\left(h_{k} a_{\varphi}^{k-j}\right) \log ^{j} x \\
& =\sum_{j=0}^{k} \sum_{k=j}^{f}(-1)^{k-j} p^{-k}\left(\begin{array}{c}
k \\
j
\end{array}\right) \psi\left(h_{k}\left(p a_{\varphi}\right)^{k-j}\right) \log ^{j} x .
\end{aligned}
$$

Comme $p a_{\varphi} \in \mathcal{H}_{0}^{[\rho, \infty)}, \psi(h)$ appartient à $p^{-f} \mathcal{H}_{0}^{[\rho, \infty)}[\log x]$, ce qui démontre la première assertion pour $n=1$. Comme $\psi(h)$ est encore de degré $\leqslant f$ en log $x$, le cas $n$ quelconque s'en déduit. Montrons la deuxième assertion. Les $h_{k}$ sont alors dans $\mathcal{B}$.

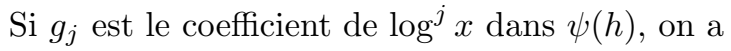

$$
g_{j}=\sum_{k=j}^{f}(-1)^{k-j} p^{-k}\left(\begin{array}{c}
k \\
j
\end{array}\right) \psi\left(h_{k}\left(p a_{\varphi}\right)^{k-j}\right)
$$

et en utilisant le lemme 1.1.6

$$
\begin{aligned}
\left\|g_{j}\right\|_{\rho} \leqslant \sup _{k}\left(p^{k}\left\|\psi\left(h_{k}\left(p a_{\varphi}\right)^{k-j}\right)\right\|_{\rho}\right) & \leqslant p^{f+1} \sup _{k}\left(\left\|h_{k}\left(p a_{\varphi}\right)^{k-j}\right\|_{\rho^{1 / p}}\right) \\
& \leqslant p^{f+1} \sup _{k}\left(\left\|h_{k}\right\|_{\rho^{1 / p}}\right)
\end{aligned}
$$

On a en fait trouvé l'inégalité plus précise suivante

$$
\sup _{j}\left(p^{n j}\left\|g_{j}\right\|_{\rho_{n}}\right) \leqslant p \sup _{k}\left(p^{(n+1) k}\left\|h_{k}\right\|_{\rho_{n+1}}\right)
$$

avec $\rho_{n}=\rho^{1 / p^{n}}$. Aussi, est-il raisonnable de donner à $\left\|\log ^{k} x\right\|_{\rho_{n}}$ une valeur équivalente à $p^{n k}$ lorsque $n$ varie. Ceci justifie la définition qui suit : pour $g \in \mathcal{B}[\log x]$, $g=\sum_{j} g_{j} \log ^{j} x$, on pose

$$
\|g\|^{(n)}=\sup _{j}\left(p^{n j}\left\|g_{j}\right\|_{\rho_{n}}\right)
$$

avec $\rho_{n}=p^{-1 /(p-1) p^{n}}$. Si $g \in \mathcal{B},\|g\|^{(n)}$ est simplement $\|g\|_{\rho_{n}}$. Le lemme 1.2 .5 et sa démonstration impliquent alors que

$$
\begin{array}{r}
\|\psi(h)\|^{(n)} \leqslant\|\psi(h)\|^{(n+1)} \text { pour } h \in \mathcal{B}_{-}[\log x] \\
\|\psi(h)\|^{(n)} \leqslant p\|\psi(h)\|^{(n+1)} \text { pour } h \in \mathcal{B}[\log x]
\end{array}
$$

ce qui est l'analogue des formules du lemme 1.1.6.

1.2.6. Un élément $h$ de $\mathcal{B}$ est dit d'ordre $\leqslant u^{-}($resp. $\leqslant u)$ si $p^{-n u}\|h\|_{\rho^{1 / p^{n}}}$ tend vers 0 (resp. est bornée) lorsque $n \rightarrow \infty ; h=\sum_{k=0}^{r} h_{k} \log ^{k} x \in \mathcal{B}[\log x]$ est dit d'ordre $\leqslant u^{-}$ $($ resp. $\leqslant u)$ si $h_{k}$ est d'ordre $\leqslant(u-k)^{-}($resp. $\leqslant u-k)$ pour tout $k$. Autrement dit, $h \in \mathcal{B}[\log x]$ est d'ordre $\leqslant u^{-}($resp. $\leqslant u)$ si et seulement si $p^{-n u}\|h\|^{(n)}$ tend vers 0 (resp. est bornée) lorsque $n \rightarrow \infty$. On note $\mathfrak{o}(h)$ la borne inférieure des $u$ tels que $H$ soit d'ordre $\leqslant u\left(\right.$ ou $\left.\leqslant u^{-}\right)$. 
Plus généralement, si $\mathcal{D}$ est un $\varphi$-module de dimension finie munie d'une norme, un élément $H$ de $\mathcal{B} \otimes \mathcal{D}$ est dit d'ordre $\leqslant u^{-}$(resp. $\leqslant u$ ) si $p^{-n u}\left\|(1 \otimes \varphi)^{-n} H\right\|^{(n)}$ tend vers 0 (resp. est bornée) lorsque $n \rightarrow \infty$ et qu'un élément $H=\sum_{k=0}^{r} H_{k} \log ^{k} x \in \mathcal{B}[\log x] \otimes \mathcal{D}$ est d'ordre $\leqslant u^{-}($resp. $\leqslant u)$ si $p^{-n u}\left\|(1 \otimes \varphi)^{-n} H\right\|^{(n)}$ tend vers 0 (resp. est bornée) lorsque $n \rightarrow \infty$, c'est-à-dire si et seulement si $H_{k}$ est d'ordre $\leqslant(u-k)^{-}($resp. $\leqslant u-k)$ pour tout $k$. On appelle ordre de $H$, et on note $\mathfrak{o}_{\varphi}(H)$, la borne inférieure des $u$ tels que $H$ soit d'ordre $\leqslant u$ (on parlera quelquefois de $\varphi$-ordre).

Nous utiliserons les $\|\cdot\|_{\rho}$ pour montrer la convergence de suites d'éléments de $\mathcal{B}[\log x]$ et les $\|\cdot\|^{(n)}$ pour calculer leur ordre qui mesure leur croissance au bord.

1.2.7. On note $N$ l'unique dérivation de $\mathcal{B}[\log x]$ sur $\mathcal{B}$ telle que $N \log x=1$. On a $N \varphi=p \varphi N, N \tau=\tau N$ pour tout $\tau \in G_{\infty}$, car $N a_{\varphi}=0$ et $N a_{\tau}=0$. L'opérateur $N$ est continu sur $\mathcal{B}[\log x]$.

1.2.8. On note $D$ la dérivation sur $\mathcal{B}$ donnée par $D(f)=(1+x) f^{\prime}(x)$. On note encore $D$ la dérivation de $\mathcal{B}[\log x]$ prolongeant $D$ et telle que

$$
D(\log x)=\frac{x+1}{x}=1+\frac{1}{x} \in x^{-1} \mathbb{Z}_{p} \llbracket x \rrbracket \subset \mathcal{B} .
$$

\subsubsection{Lemme. - L'opérateur $D$ de $\mathcal{B}[\log x]$ est surjectif et de noyau $\mathbb{Q}_{p}$.}

Démonstration. - Soit $\sum_{j=0}^{r} f_{j} \log ^{j} x$ un élément du noyau de $D$. Comme log $x$ n'est pas algébrique sur $\mathcal{B}$, on a nécessairement les relations (avec $f_{r+1}=0$ )

$$
D\left(f_{j}\right)+(j+1) \frac{x+1}{x} f_{j+1}=0 .
$$

Pour $j=r$, on obtient que $f_{r}$ est une constante $\lambda$. Pour $r>0$, on a alors $D\left(f_{r-1}\right)=-\lambda r(x+1 / x)$. Comme $(x+1) / x$ n'est pas la dérivée d'un élément de $\mathcal{B}$, cela implique que $\lambda=0$. Donc $r=0$ et le noyau de $D$ est $\mathbb{Q}_{p}$. Montrons la surjectivité de $D$. Comme $D$ diffère de la dérivation usuelle par multiplication par une unité de $\mathbb{Z}_{p} \llbracket x \rrbracket$, il suffit de montrer que tout élément de $\mathcal{B}[\log x]$ est intégrable. On commence par les $x^{r} \log ^{s} x$ pour $s \geqslant 0$. On a la formule

$$
\int x^{r} \log ^{s} x=\frac{x^{r+1}}{(r+1)^{s+1}} \sum_{j=0}^{s}(-1)^{s-j} \frac{s !}{j !}(r+1)^{j} \log ^{j} x
$$

pour $r \neq-1$ et

$$
\int x^{-1} \log ^{s} x=\frac{\log ^{s+1} x}{s+1} .
$$

On en déduit qu'il existe un élément $F_{r, s}$ de $x^{r+1} \mathbb{Q}_{p}[\log x]$ tel que $F_{r, s}^{\prime}=x^{r} \log ^{s} x$ et les coefficients $a_{r+1, k}\left(F_{r, s}\right)$ de $x^{r+1} \log ^{k} x$ pour $k \leqslant s+1$ vérifient

$$
\left|a_{r+1, k}\left(F_{r+1, s}\right)\right| \leqslant \begin{cases}|r+1|^{-(s+1-k)} & \text { si } r \neq-1 \\ |s+1|^{-1} & \text { si } r=-1 \text { et } k=s+1 .\end{cases}
$$


Si maintenant $f=\left(\sum_{r} b_{r}(f) x^{r}\right) \log ^{s} x$ est un élément de $\mathcal{H}^{\left[\rho, \rho^{\prime}\right]} \log ^{s} x$ pour $0 \leqslant \rho<\rho^{\prime} \leqslant 1$, formellement $F=\sum_{r} b_{r}(f) F_{r, s}$ vérifie $F^{\prime}=f$. Vérifions que $F$ appartient à $\mathcal{B}[\log x]$. Si $a_{r, k}(F)$ est le coefficient de $x^{r} \log ^{k} x$ dans $F$ pour $r \in \mathbb{Z}$ et $0 \leqslant k \leqslant s+1$, on a pour $\rho<\rho^{\prime \prime}<\rho^{\prime}$ et pour $r \neq 0$

$$
\left|a_{r, k}(F)\right| \rho^{\prime \prime r} \leqslant\left|b_{r-1}(f)\right| \rho^{\prime \prime r} /|r|^{s+1-k} \leqslant \begin{cases}\left|b_{r-1}(f)\right| \rho^{r} \cdot \frac{\left(\rho / \rho^{\prime \prime}\right)^{-r}}{|r|^{s+1-k}} & \text { si } r \leqslant 0 \\ \left|b_{r-1}(f)\right| \rho^{\prime r} \cdot \frac{\left(\rho^{\prime \prime} / \rho^{\prime}\right)^{r}}{|r|^{s+1-k}} & \text { si } r \geqslant 0\end{cases}
$$

Comme $\lim _{r \rightarrow-\infty}\left|b_{r-1}(f)\right| \rho^{r}=0$, que $\lim _{r \rightarrow+\infty}\left|b_{r-1}(f)\right| \rho^{\prime r}=0$ et que si $0 \leqslant \alpha<1$, $\lim _{n \rightarrow+\infty} \alpha^{n} /|n|^{s+1-k}=0$ (la série $\sum_{n} x^{n} / n^{s+1-k}$ converge pour $|x|<1$ ), on en déduit que

$$
\lim _{r \rightarrow \pm \infty}\left|a_{r, k}(F)\right| \rho^{\prime \prime r}=0
$$

et donc que $F$ est un élément de $\mathcal{H}^{] \rho, \rho^{\prime}}\left[[\log x]\right.$ et donc un élément de $\mathcal{B}^{\prime}[\log x]$. Montrons qu'il appartient aussi à $\mathcal{B}[\log x]$, s'il en est de même de $f$.

Pour $r<0$, on a avec $\rho_{n}=\rho^{\prime \prime 1 / p^{n}}$,

$$
\left|a_{r, k}(F)\right| \rho_{n}^{r} \leqslant\left|a_{r, k}(F)\right| \rho^{\prime \prime r}
$$

ce qui est borné et dès que $h>0$, on a

$$
\lim _{n \rightarrow \infty}\left|p^{n h} a_{r, k}(F)\right| \rho_{n}^{r}=0 .
$$

Pour $r \geqslant 0$, on a

$$
\left|a_{r, k}(F)\right| \rho_{n}^{r} \leqslant\left|b_{r-1}(f)\right| \rho_{n+1}^{r} \frac{\left(\rho_{n} / \rho_{n+1}\right)^{r}}{|r|^{s+1-k}}
$$

et pour $r<0$,

$$
\left|a_{r, k}(F)\right| \rho_{n}^{r} \leqslant\left|b_{r-1}(f)\right| \rho_{n-1}^{r} \frac{\left(\rho_{n} / \rho_{n-1}\right)^{r}}{|r|^{s+1-k}}
$$

Soit $M_{n}=\sup _{r}\left(\left(\rho_{n} / \rho_{n+1}\right)^{r} /|r|^{s+1-k}\right)$ pour $r \geqslant 0$ et $M_{n}=\sup _{r}\left(\left(\rho_{n} / \rho_{n-1}\right)^{r} /|r|^{s+1-k}\right)$ pour $r<0$. Par des arguments classiques, le maximum est atteint pour un entier $r_{0}=p^{t_{0}}$ avec $n+A \leqslant t_{0}<n+1+A$ et

$$
A=\frac{\log ((s+1-k) /(p-1))}{\log p}
$$

une constante indépendante de $n$. On en déduit que

$$
M_{n} \leqslant B p^{n(s+1-k)}
$$

avec $B$ indépendant de $n$. Ainsi,

$$
\left|a_{r, k}(F)\right| \rho_{n}^{r} \leqslant B\left|b_{r-1}(f)\right| \rho_{n+1}^{r-1} p^{n(s+1-k)} .
$$

On en déduit que si $f$ appartient à $\mathcal{B}[\log x]$, il en est de même de $F$ et on a même $\mathfrak{o}(F) \leqslant \mathfrak{o}(f)+1$. 
1.2.10. Corollaire. - L'opérateur $D^{k}$ de $\mathcal{B}[\log x]$ est surjectif. Son noyau est le $\mathbb{Q}_{p}$-espace vectoriel engendré par les $\log ^{r}(1+x)$ pour $0 \leqslant r<k$ et est donc contenu dans $\mathcal{H}$.

1.2.11. Lemme. - Le noyau de $\varphi-p^{r}$ sur $\mathcal{B}[\log x]$ est égal à 0 si $r<0$ et à $\mathbb{Q}_{p} \log ^{r}(1+x)$ si $r \geqslant 0$.

Démonstration. - Le fait que le noyau de $\varphi-p^{r}$ sur $\mathcal{B}_{-}$soit nul pour tout entier $r \in \mathbb{Z}$ se déduit de ce que $\varphi\left(1 / x^{n}\right)=1 / x^{p n}+\left(1 / x^{p n+1}\right) \mathbb{Z}_{p} \llbracket 1 / x \rrbracket$. Comme $\varphi$ laisse stable $\mathcal{B}_{-}$et $\mathcal{B}_{+}=\mathcal{H}$, on en déduit que le noyau de $\varphi-p^{r}$ sur $\mathcal{B}$ est égal au noyau de $\varphi-p^{r}$ sur $\mathcal{H}$ et il est bien connu que ce dernier est $\mathbb{Q}_{p} \log ^{r}(1+x)[\mathbf{1 9}, 2.2]$. Passons à $\mathcal{B}[\log x]$. Soit $F=\sum_{j=0}^{s} F_{j} \log ^{j} x$ avec les $F_{j}$ dans $\mathcal{B}, F_{s} \neq 0$ et $s>0$. Si $\varphi(F)=p^{r} F$, on a $p^{s} \varphi\left(F_{s}\right)=p^{r} F_{s} \operatorname{car} \varphi(\log x)-p \log x$ appartient à $\mathcal{B}$. On en déduit que $\varphi\left(F_{s}\right)=p^{r-s} F_{s}$ avec $F_{s} \in \mathcal{B}$. Si $r<s, F_{s}=0$; donc $F=0$. En particulier si $r \leqslant 0$ et $\varphi(F)=p^{r} F$, $F$ est nul. Si $r>0$, en appliquant l'opérateur de dérivation $D^{r}$, on en déduit que $D^{r}(F)$ est nul. En appliquant le corollaire 1.2.10, $F=\sum_{i=0}^{r} a_{i} \log ^{i}(1+x) \in \mathcal{H}$ et on en déduit le lemme.

\subsection{Structure de $G_{\infty}$-modules}

Posons $\alpha=p^{-1} \varphi \log x$ et $\alpha_{\gamma}=(\gamma-1) \alpha$ pour $\gamma \in \Gamma$.

1.3.1. Proposition. - Il existe une unique structure naturelle de $\mathcal{H}\left(G_{\infty}\right)$-module sur $\mathcal{B}[\log x]$ prolongeant l'action de $G_{\infty}$. De plus, on a pour $\lambda \in \mathcal{H}(\Gamma)$ et $f \in \mathcal{B}[\log x]$ de degré $r$,

$$
\|\lambda \cdot f\|_{\rho} \leqslant C(\rho, r)\|\lambda\|_{\rho^{p-1}}\|f\|_{\rho}
$$

avec $C(\rho, r)=\sup _{0 \leqslant k \leqslant r}\left(\left\|\alpha_{\gamma}\right\|_{\rho}^{k} \rho^{-k(p-1)}\right)$.

Commençons par quelques lemmes.

1.3.2. Lemme. - Pour $\gamma \in \Gamma$ avec ord $(\chi(\gamma)-1)=m \geqslant 1$ et $\rho>\rho_{m-1}$, pour $n$ et $k$ des entiers positifs,

$$
\left\|(\gamma-1)^{n}\left(\alpha^{k}\right)\right\|_{\rho} \leqslant \rho^{\left(p^{m}-1\right) \sup (n-k, 0)}\left\|\alpha_{\gamma}\right\|_{\rho}^{k} .
$$

Démonstration. - Prenons d'abord $k=1$. Alors, $(\gamma-1)(\alpha)=\alpha_{\gamma}$. Comme $\alpha_{\gamma} \in \mathcal{H} \subset \mathcal{B}$, on a d'après (1.1.1) (démonstration de la proposition 1.1.7)

$$
\left\|(\gamma-1)^{n}(\alpha)\right\|_{\rho}=\left\|(\gamma-1)^{n-1} \alpha_{\gamma}\right\|_{\rho} \leqslant \rho^{\left(p^{m}-1\right)(n-1)}\left\|\alpha_{\gamma}\right\|_{\rho} .
$$


D'où la majoration désirée de $\left\|(\gamma-1)^{n}(\alpha)\right\|_{\rho}$. Supposons le lemme pour les entiers $j \leqslant k$. On a alors

$$
\begin{aligned}
(\gamma-1)^{n}\left(\alpha^{k+1}\right) & =(\gamma-1)^{n-1}\left(\gamma \alpha^{k+1}-\alpha^{k+1}\right) \\
& =(\gamma-1)^{n-1}\left(\sum_{j=0}^{k}\left(\begin{array}{c}
k+1 \\
j
\end{array}\right) \alpha_{\gamma}^{k+1-j} \alpha^{j}\right) .
\end{aligned}
$$

Or

$$
(\gamma-1)^{s}(f g)=\sum_{i=0}^{s}\left(\begin{array}{l}
s \\
i
\end{array}\right) \gamma^{s-i}(\gamma-1)^{i}(f)(\gamma-1)^{s-i}(g)
$$

On a donc

$(\gamma-1)^{n}\left(\alpha^{k+1}\right)=\sum_{j=0}^{k} \sum_{i=0}^{n-1}\left(\begin{array}{c}k+1 \\ j\end{array}\right)\left(\begin{array}{c}n-1 \\ i\end{array}\right)(\gamma-1)^{i}\left(\gamma^{n-1-i}\left(\alpha_{\gamma}^{k+1-j}\right)(\gamma-1)^{n-i-1}\left(\alpha^{j}\right)\right.$.

D'où la majoration

$$
\left\|(\gamma-1)^{n}\left(\alpha^{k+1}\right)\right\|_{\rho} \leqslant \sup _{0 \leqslant j \leqslant k, 0 \leqslant i \leqslant n-1} \rho^{i\left(p^{m}-1\right)}\left\|\alpha_{\gamma}\right\|_{\rho}^{k+1-j}\left\|(\gamma-1)^{n-i-1}\left(\alpha^{j}\right)\right\|_{\rho} .
$$

En utilisant l'hypothèse de récurrence,

$$
\rho^{i\left(p^{m}-1\right)}\left\|\alpha_{\gamma}\right\|_{\rho}^{k-j}\left\|(\gamma-1)^{n-i-1}\left(\alpha^{j}\right)\right\|_{\rho} \leqslant \begin{cases}\rho^{(n-j-1)\left(p^{m}-1\right)}\left\|\alpha_{\gamma}\right\|_{\rho}^{k+1} & \text { si } n-i-1 \geqslant j \\ \rho^{i\left(p^{m}-1\right)}\left\|\alpha_{\gamma}\right\|_{\rho}^{k+1} & \text { si } n-i-1<j .\end{cases}
$$

Donc,

$$
\rho^{i\left(p^{m}-1\right)}\left\|\alpha_{\gamma}\right\|_{\rho}^{k-j}\left\|(\gamma-1)^{n-i-1}\left(\alpha^{j}\right)\right\|_{\rho} \leqslant \rho^{\sup (i, n-1-j)}\left\|\alpha_{\gamma}\right\|_{\rho}^{k+1}
$$

Comme $j \leqslant k$ et $i \geqslant 0, n-1-j \geqslant n-1-k$ et $\sup (i, n-1-j) \leqslant \sup (n-k-1,0)$. Finalement,

$$
\left\|(\gamma-1)^{n}\left(\alpha^{k+1}\right)\right\|_{\rho} \leqslant \rho^{\sup (n-k-1,0)\left(p^{m}-1\right)}\left\|\alpha_{\gamma}\right\|_{\rho}^{k+1} .
$$

1.3.3. Lemme. - On a pour $g \in \mathcal{H}^{[\eta, 1[}$ et $\rho>\sup \left(\eta, \rho_{m-1}\right)$

$$
\left\|(\gamma-1)^{n}\left(g \alpha^{k}\right)\right\|_{\rho} \leqslant\|g\|_{\rho}\left\|\alpha_{\gamma}\right\|_{\rho}^{k} \rho^{\left(p^{m}-1\right) \sup (n-k, 0)} .
$$

Démonstration. - On applique l'identité (1.3.1) à $f=\alpha^{k}$ et l'équation (1.1.1) (démonstration de la proposition 1.1.7) :

$$
\left\|(\gamma-1)^{n}\left(g \alpha^{k}\right)\right\|_{\rho} \leqslant \sup _{0 \leqslant i \leqslant n} \rho^{\left(p^{m}-1\right)(\sup (i-k, 0)+n-i)}\left\|\alpha_{\gamma}\right\|_{\rho}^{k}\|g\|_{\rho} .
$$

Or

$$
\begin{aligned}
\sup _{0 \leqslant i \leqslant n} \rho^{\left(p^{m}-1\right) \sup (n-k, n-i)} & =\rho^{\left(p^{m}-1\right) \inf _{0 \leqslant i \leqslant n} \sup (n-k, n-i)} \\
& =\rho^{\left(p^{m}-1\right) \sup (0, n-k)}
\end{aligned}
$$

D'où le lemme. 
Démonstration de la proposition 1.3.1. - La convergence de $\lambda \cdot f$ pour $\lambda \in \mathcal{H}(\Gamma)$ et $f \in \mathcal{B}[\log x]$ se déduit du lemme précédent. On a de plus pour $g \in \mathcal{B}$ et $\lambda=\sum_{n} a_{n}(\gamma-1)^{n}$

$$
\begin{aligned}
\left\|\lambda \cdot\left(g \alpha^{k}\right)\right\|_{\rho} & \leqslant\left\|a_{\gamma}\right\|_{\rho}^{k}\|g\|_{\rho} \sup _{n}\left|a_{n}\right| \rho^{(p-1)(n-\inf (k, n))} \\
& \leqslant\left\|a_{\gamma}\right\|_{\rho}^{k}\|g\|_{\rho}\|\lambda\|_{\rho^{p-1}} \rho^{-(p-1) k}
\end{aligned}
$$

D'où, pour $g \in \mathcal{B}[\log x]$ de degré $r$

$$
\|\lambda \cdot g\|_{\rho} \leqslant C(\rho, r)\|\lambda\|_{\rho^{p-1}}\|f\|_{\rho} .
$$

Il reste à vérifier que $\lambda \cdot f$ appartient bien à $\mathcal{B}[\log x]$ (et pas seulement à $\mathcal{B}^{\prime}[\log x]$ ). Pour cela, il suffit d'appliquer les définitions et de remarquer que les $p^{n r} C\left(\rho^{1 / p^{n}}, r\right)$ sont bornés car $\mathfrak{o}\left(a_{\gamma}\right)=1$.

1.3.4. Proposition. - L'anneau $\mathcal{B}[\log x]^{\psi=0}$ est muni d'une structure de $\mathcal{B}\left(G_{\infty}\right)$ module libre. Il admet comme base les $(1+x) p^{-i} \varphi \log ^{i} x$.

En particulier, si $\tau \in G_{\infty}$ n'est pas de torsion, $\tau-1$ est inversible sur $\mathcal{B}[\log x]^{\psi=0}$.

Démonstration. - Par le théorème 1.1.8, si $\tau \in \Gamma$ n'est pas l'identité, $\tau-1$ est injectif sur $\mathcal{B}^{\psi=0}$. Il est aussi injectif sur $\mathcal{B}[\log x]^{\psi=0}$ : en effet si $f$ appartient au noyau, comme $(\tau-1) \log ^{k} x$ est de degré $<k$, le coefficient de plus haut degré de $f$ (comme polynôme en $\log x$ ) est dans le noyau de $\tau-1$ et est donc nul. Montrons ensuite par récurrence sur le degré que $\tau-1$ est surjective sur $\mathcal{B}[\log x]^{\psi=0}$ pour $\tau \in \Gamma$. Soit $m=\operatorname{ord}(\chi(\tau)-1)$. Posons toujours $\alpha=p^{-1} \varphi \log x$. L'hypothèse de récurrence étant que les éléments de $\mathcal{B}[\log x]^{\psi=0}$ de degré $<k$ sont dans l'image de $\tau-1$, il suffit de montrer que les $f_{k} \alpha^{k}$ avec $f_{k} \in \mathcal{B}^{\psi=0}$ sont dans l'image de $\tau-1$. Cherchons $G \in \mathcal{B}[\log x]^{\psi=0}$ tel que

$$
(\tau-1) G=f_{k} \alpha^{k}
$$

sous la forme $G=g_{k} \alpha^{k}+G_{1}$ avec $g_{k} \in \mathcal{B}^{\psi=0}$ et $G_{1}$ de degré $k-1$; l'équation devient

$$
f_{k} \alpha^{k}=(\tau-1)\left(g_{k}\right) \alpha^{k}+\tau\left(g_{k}\right)(\tau-1) \alpha^{k}+(\tau-1) G_{1} .
$$

Comme $(\tau-1) \alpha^{k}$ est de degré $<k$, elle est équivalente à

$$
\begin{aligned}
(\tau-1) g_{k} & =f_{k} \\
(\tau-1) G_{1} & =-\tau\left(g_{k}\right)(\tau-1) \alpha^{k} .
\end{aligned}
$$

En appliquant l'hypothèse de récurrence, on en déduit que ces équations en $g_{k}$ et $G_{1}$ ont une solution.

Calculons $\left\|(\tau-1)^{-n}(F)\right\|_{\rho}$ pour $F$ de degré $k$ : montrons par récurrence que pour $n \geqslant 0$

$$
\left\|(\tau-1)^{-n}(F)\right\|_{\rho} \leqslant \rho^{-n p^{m}} \rho^{c(k) p^{m}} \sup \left(1,\left\|\alpha_{\tau}\right\|_{\rho}\right)^{c(k)}\|F\|_{\rho}
$$


avec $c(k)$ un entier ne dépendant que de $k$. La démonstration est analogue au calcul de $\left\|(\tau-1)^{n}(F)\right\|_{\rho}$. Pour $k=0$, il s'agit de montrer que

$$
\left\|(\tau-1)^{-n}(F)\right\|_{\rho} \leqslant \rho^{-n p^{m}}\|F\|_{\rho},
$$

ce qui est démontré dans l'appendice (on a donc $c(0)=0$ ). Supposons-le vrai pour $k$. Il suffit de prendre $F=f_{k+1} \alpha^{k+1}$. Si $G=(\tau-1)^{-n} F=g_{k+1} \alpha^{k+1}+G_{1}$ avec $G_{1}$ de degré $\leqslant k$, on a

$$
(\tau-1)^{n}\left(g_{k+1}\right)=f_{k+1}
$$

Donc

$$
\left\|g_{k+1}\right\|_{\rho} \leqslant \rho^{-n p^{m}}\left\|f_{k+1}\right\|_{\rho}=\rho^{-n p^{m}}\|f\|_{\rho}
$$

De plus

$$
(\tau-1)^{n}\left(G_{1}\right)=-\sum_{i=0}^{n-1}\left(\begin{array}{c}
n \\
i
\end{array}\right) \tau^{n-i}(\tau-1)^{i} g_{k+1}(\tau-1)^{n-i}\left(\alpha^{k+1}\right) .
$$

Si $F_{k}$ est le second membre, on a

$$
\begin{aligned}
\left\|F_{k}\right\|_{\rho} & \leqslant \sup _{0 \leqslant i \leqslant n-1} \rho^{i p^{m}}\left\|g_{k+1}\right\|_{\rho} \rho^{\sup (0, n-i-k-1) p^{m}}\left\|\alpha_{\tau}\right\|_{\rho}^{k+1} \\
& \leqslant \sup _{0 \leqslant i \leqslant n-1} \rho^{\sup (i, n-k-1) p^{m}}\left\|\alpha_{\tau}\right\|_{\rho}^{k+1}\left\|g_{k+1}\right\|_{\rho} \\
& \leqslant \rho^{(n-k-1) p^{m}}\left\|\alpha_{\tau}\right\|_{\rho}^{k+1}\left\|g_{k+1}\right\|_{\rho} \\
& \leqslant \rho^{-(k+1) p^{m}}\left\|\alpha_{\tau}\right\|_{\rho}^{k+1}\|F\|_{\rho}
\end{aligned}
$$

en supposant que $n>k$. Donc,

$$
\begin{aligned}
\left\|G_{k}\right\|_{\rho} & \leqslant \rho^{-n p^{m}} \rho^{-c(k) p^{m}} \sup \left(1,\left\|\alpha_{\tau}\right\|_{\rho}\right)^{c(k)}\left\|F_{k}\right\|_{\rho} \\
& \leqslant \rho^{-n p^{m}} \rho^{-(c(k)+k+1) p^{m}} \sup \left(1,\left\|\alpha_{\tau}\right\|_{\rho}\right)^{c(k)+k+1}\|F\|_{\rho} .
\end{aligned}
$$

Comme $\rho^{-(c(k)+k+1) p^{m}} \sup \left(1,\left\|\alpha_{\tau}\right\|_{\rho}\right)^{c(k)+k+1}$ est supérieur à 1 , on en déduit que

$$
\|g\|_{\rho} \leqslant \rho^{-n p^{m}} \rho^{-c(k+1) p^{m}} \sup \left(1,\left\|\alpha_{\tau}\right\|_{\rho}\right)^{c(k+1)}\|F\|_{\rho}
$$

avec $c(k+1)=c(k)+k+1$, c'est-à-dire que $c(k)=k(k+1) / 2$.

On déduit facilement de l'inégalité 1.3.2 que l'action de $G_{\infty} \operatorname{sur} \mathcal{B}[\log x]^{\psi=0}$ se prolonge à $\mathcal{B}\left(G_{\infty}\right)$.

1.3.5. Exemple : Polylogarithmes. - On peut lire ici la première partie de l'appendice A. Donnons juste les notations suivantes qui en sont tirées. Posons

$$
\mathfrak{L}_{0}(x)=\frac{x+1}{x}, \quad \mathfrak{L}_{1}(x)=\log x .
$$

Il existe une unique suite $\left(\mathfrak{L}_{k}\right)_{k \in \mathbb{Z}}$ d'éléments de $\mathcal{B}[\log x]$ telle que

$$
D\left(\mathfrak{L}_{k}\right)=\mathfrak{L}_{k-1}, \quad \psi\left(\mathfrak{L}_{k}\right)=p^{-k} \mathfrak{L}_{k} .
$$


Pour $k \geqslant 1$,

$$
N \mathfrak{L}_{k}(x)=\frac{\log ^{k-1}(1+x)}{(k-1) !}
$$

et

$$
\mathfrak{L}_{k}(x)-\left(\frac{\log ^{k-1}(1+x)}{(k-1) !}\right) \log x
$$

est un élément de $\mathcal{H}$.

Enfin, pour $k \in \mathbb{Z}, \mathfrak{L}_{k}^{(p)} \stackrel{\text { déf }}{=}\left(1-p^{-k} \varphi\right) \mathfrak{L}_{k}$ appartient à $\mathcal{B}_{-}^{\psi=0}$ et vaut 0 en $\infty$. Plus précisément, $\mathfrak{L}_{k}^{(p)}$ est défini pour $|x|>p^{-1 /(p-1)}$. Enfin, $\mathfrak{L}_{0}^{(p)}$ est un avatar de la fonction de Kubota-Leopoldt. 



\section{CHAPITRE 2}

\section{MODULES D'IWASAWA ASSOCIÉS À UN $(\varphi, N)$-MODULE}

\section{1. $(\varphi, N)$-modules}

On appelle $(\varphi, N)$-module un $\mathbb{Q}_{p}$-espace vectoriel de dimension finie muni de deux opérateurs $\varphi$ et $N$ vérifiant $N \varphi=p \varphi N$. Soit un $(\varphi, N)$-module $\mathcal{D}$. On a sur $\mathcal{B}[\log x] \otimes \mathcal{D}$ diverses opérations : action de $G_{\infty}$, action de $\Phi=\varphi \otimes \varphi$, action de $N=1 \otimes N+N \otimes 1$. On note $\mathbb{Q}_{p}[k]$ le $(\varphi, N)$-module $\mathbb{Q}_{p}$ avec $\varphi=p^{k}, N=0$ et $\mathcal{D}[k]=\mathcal{D} \otimes \mathbb{Q}_{p}[k]$. On note $e_{k}$ la base 1 de $\mathbb{Q}_{p}[k]$ mais nous identifierons la plupart du temps $\mathcal{D}[k]$ et $\mathcal{D}$.

\section{2. Étude de la dérivation}

Soit $\mathcal{D}$ un $(\varphi, N)$-module de dimension finie. On pose

$$
\mathcal{D}_{\infty}^{\text {gros }}(\mathcal{D})=\left(\mathcal{B}[\log x]^{\psi=0} \otimes \mathcal{D}\right)^{N=0} .
$$

2.2.1. Proposition. - Le $\mathcal{B}\left(G_{\infty}\right)$-module $\mathcal{D}_{\infty}^{\text {gros }}(\mathcal{D})$ est un $\mathcal{B}\left(G_{\infty}\right)$-module libre de type fini de rang égal à $\operatorname{dim}_{\mathbb{Q}_{p}} \mathcal{D}$.

Pour la démonstration, introduisons pour $\alpha \in \mathcal{B}[\log x]$ l'homomorphisme de $\mathbb{Q}_{p^{-}}$ espaces vectoriels

$$
\mathcal{E}_{\alpha}: \mathcal{B} \otimes \mathcal{D} \rightarrow \mathcal{B}[\log x] \otimes \mathcal{D}
$$

défini par

$$
\mathcal{E}_{\alpha}(f)=\exp (-\alpha \cdot N)(f)=\sum_{k=0}^{\infty} \frac{(-1)^{k}}{k !} N^{k}(f) \alpha^{k} .
$$

2.2.2. Lemme. - Si $N \alpha=1, \mathcal{E}_{\alpha}$ est à valeurs dans $(\mathcal{B}[\log x] \otimes \mathcal{D})^{N=0}$ et

$$
\mathcal{E}_{\alpha}(f) \equiv f \bmod \mathcal{B}[\log x] \otimes N \mathcal{D} .
$$


L'application réciproque est donnée par $\Delta_{\alpha}(f)=\exp (\alpha \cdot(1 \otimes N))(f)$. Lorsque $N f=0$, $\Delta_{\alpha}(f)$ est aussi le terme constant de $f$ dans son développement comme polynôme en $\alpha$ :

$$
f=\sum_{k \geqslant 0} \frac{(-1)^{k}}{k !} N^{k}\left(\Delta_{\alpha}(f)\right) \alpha^{k} .
$$

La démonstration est facile : par exemple,

$$
N\left(\mathcal{E}_{\alpha}(f)\right)=-N(\alpha)(1 \otimes N) \exp (-\alpha(1 \otimes N))(f)+\exp (-\alpha(1 \otimes N))((1 \otimes N) f)=0 .
$$

Prenons maintenant $\alpha=p^{-1} \varphi \log x$. Si $d$ est un élément de $\mathcal{D}, \mathcal{E}_{\alpha}((1+x) d)$ est alors un élément de $\mathcal{D}_{\infty}^{\text {gros }}(\mathcal{D})$. Il est clair que l'image de $\mathcal{D}$ par l'application $d \mapsto \mathcal{E}_{\alpha}((1+x) d)$ engendre un $\mathcal{B}\left(G_{\infty}\right)$-module de rang $d=\operatorname{dim}_{\mathbb{Q}_{p}} \mathcal{D}$. Bien que $\mathcal{E}_{\alpha}$ ne soit pas un isomorphisme de $G_{\infty}$-modules, par récurrence sur le degré de nilpotence de $\mathcal{D}$, on vérifie que

$$
\mathcal{D}_{\infty}^{\text {gros }}(\mathcal{D})=\mathcal{B}\left(G_{\infty}\right) \mathcal{E}_{\alpha}((1+x) \mathcal{D})
$$

et que si $\left(d_{i}\right)$ est une base de $\mathcal{D}$ (par exemple adaptée à la filtration par les sous-espaces $\left.N^{r} \mathcal{D}\right)$, les $\mathcal{E}_{\alpha}\left((1+x) d_{i}\right)$ forment une base de $\mathcal{D}_{\infty}^{\text {gros }}(\mathcal{D})$.

2.2.3. La dérivation $D$ induit un opérateur $D$ de $G_{\infty}$-modules de $\mathcal{D}_{\infty}^{\text {gros }}(\mathcal{D})$ sur $\mathcal{D}_{\infty}^{\text {gros }}(\mathcal{D}[1])(1)$.

Proposition. - L'homomorphisme

$$
D: \mathcal{D}_{\infty}^{\text {gros }}(\mathcal{D}) \longrightarrow \mathcal{D}_{\infty}^{\text {gros }}(\mathcal{D}[1])(1)
$$

est un isomorphisme de $G_{\infty}$-modules.

Démonstration. - Si $f \in \mathcal{D}_{\infty}^{\text {gros }}(\mathcal{D})$ vérifie $D(f)=0$, on a $f \in \mathcal{D}$. La condition $\psi(f)=0$ implique la nullité de $f$. Soit $f \in \mathcal{D}_{\infty}^{\text {gros }}(\mathcal{D}[1])$. D'après le lemme 1.2.9, il existe $F \in \mathcal{B}[\log x] \otimes \mathcal{D}$ tel que $D(F)=f$. Les conditions $\psi(f)=0$ et $N f=0$ impliquent que $\mu=\psi(F)$ et $\lambda=N(F)$ appartiennent à $\mathcal{D}$. Comme $\psi(\mu)=\mu$, on peut remplacer $F$ par $F-\mu$ et on a alors $\psi(F)=0$. La relation $\psi N=p N \psi$ implique alors que $\psi(\lambda)=0$ et donc que $\lambda=0$. Donc $F \in \mathcal{D}_{\infty}^{\text {gros }}(\mathcal{D})$.

2.2.4. On pose

$$
\widetilde{\mathcal{D}}_{\infty}(\mathcal{D})=(\mathcal{B}[\log x] \otimes \mathcal{D})^{N=0, \psi=1 \otimes \varphi} .
$$

La dérivation $D$ induit un opérateur

$$
D: \widetilde{\mathcal{D}}_{\infty}(\mathcal{D}[-1])(-1) \longrightarrow \widetilde{\mathcal{D}}_{\infty}(\mathcal{D}) \text {. }
$$

Notons $\delta_{0}: \mathcal{D} \rightarrow \mathcal{D} \times \mathcal{D}$ et $\delta_{1}: \mathcal{D} \times \mathcal{D} \rightarrow \mathcal{D}$ les applications définies par

$$
\begin{aligned}
\delta_{0}(\nu) & =(N \nu,(1-\varphi) \nu) \\
\delta_{1}(\lambda, \mu) & =N \mu-(1-p \varphi) \lambda .
\end{aligned}
$$


Soit $\mathfrak{C}(\mathcal{D})$ le complexe de longueur 2 :

$$
0 \longrightarrow \mathcal{D} \stackrel{\delta_{0}}{\longrightarrow} \mathcal{D} \times \mathcal{D} \stackrel{\delta_{1}}{\longrightarrow} \mathcal{D} \longrightarrow 0 \text {. }
$$

On a

$$
\begin{aligned}
& H^{0}\left(\mathfrak{C}^{\cdot}(\mathcal{D})\right)=\mathcal{D}^{\varphi=1, N=0} \\
& H^{1}\left(\mathfrak{C}^{\cdot}(\mathcal{D})\right)=\{(\lambda, \mu) \in \mathcal{D} \times \mathcal{D} \text { tel que } N \mu=(1-p \varphi) \lambda\} / \delta_{0}(\mathcal{D}) \\
& H^{2}\left(\mathfrak{C}^{\cdot}(\mathcal{D})\right)=\mathcal{D} /(N, 1-p \varphi) \mathcal{D} .
\end{aligned}
$$

On pose $\mathcal{C}(\mathcal{D})=H^{1}(\mathfrak{C} \cdot(\mathcal{D}))$. Il est facile de vérifier que $\mathcal{C}(\mathcal{D})$ fait partie d'une suite exacte

$$
\begin{array}{ccccc}
0 \longrightarrow \mathcal{D}^{N=0} /(1-\varphi) \mathcal{D}^{N=0} & \longrightarrow \mathcal{C}(\mathcal{D}) & \longrightarrow & (\mathcal{D} / N \mathcal{D})^{\varphi=p^{-1}} & \longrightarrow 0 \\
\mu & \longmapsto & (0, \mu) & & \\
& & (\lambda, \mu) & \longrightarrow & \lambda
\end{array}
$$

2.2.5. Lemme. - Soit $k$ un entier positif. On a une suite exacte de $G_{\infty}$-modules

$$
\begin{aligned}
0 \longrightarrow \oplus_{-k \leqslant i<0} H^{0}\left(\mathfrak{C}^{\cdot}(\mathcal{D}[i])(i) \longrightarrow \widetilde{\mathcal{D}}_{\infty}(\mathcal{D}[-k])(-k)\right. \\
\quad \stackrel{D^{k}}{\longrightarrow} \widetilde{\mathcal{D}}_{\infty}(\mathcal{D}) \longrightarrow \oplus_{-k \leqslant i<0} H^{1}\left(\mathfrak{C}^{\cdot}(\mathcal{D}[i])\right)(i) .
\end{aligned}
$$

En particulier, pour $r \gg 0$ ou $r \ll 0$, D est un $G_{\infty}$-isomorphisme de $\widetilde{\mathcal{D}}_{\infty}(\mathcal{D}[r])$ dans $\widetilde{\mathcal{D}}_{\infty}(\mathcal{D}[r+1])(1)$.

Ainsi pour $k=1$, on obtient la suite exacte :

$$
0 \longrightarrow \mathcal{D}^{N=0, \varphi=p}(-1) \longrightarrow \widetilde{\mathcal{D}}_{\infty}(\mathcal{D}[-1])(-1) \stackrel{D}{\longrightarrow} \widetilde{\mathcal{D}}_{\infty}(\mathcal{D}) \longrightarrow \mathcal{C}(\mathcal{D}[-1])(-1) .
$$

L'application $\widetilde{\mathcal{D}}_{\infty}(\mathcal{D}) \longrightarrow \oplus_{-k \leqslant i<0} \mathcal{C}(\mathcal{D}[i])(i)$ est décrite dans la démonstration.

Démonstration. - Si $D^{k}(f)$ est nul, $f=\sum_{0 \leqslant j<k}\left(a_{j-k} / j !\right) \log ^{j}(1+x)$. La condition $\psi(f)=p^{-k}(1 \otimes \varphi) f$ signifie alors que

$$
\sum_{0 \leqslant j<k}\left(p^{-j}-p^{-k} \varphi\right) a_{j-k} \frac{\log ^{j}(1+x)}{j !}=0
$$

et donc que $\varphi a_{j-k}=p^{k-j} a_{j-k}$. Comme $N f=0$, les $a_{j-k}$ sont dans le noyau de $N$. Donc $a_{i} \in \mathcal{D}^{N=0, \varphi=p^{-i}}$ pour $k \leqslant i<0$. Comme $G_{\infty}$ agit sur $\log ^{j}(1+x)$ par $\chi^{j}$, le noyau de $D^{k}$ est bien isomorphe à

$$
\oplus_{-k \leqslant i<0} \mathcal{D}^{N=0, \varphi=p^{-i}}(i)=\oplus_{-k \leqslant i<0} H^{0}\left(\mathfrak{C}^{\cdot}(\mathcal{D}[i])(i) .\right.
$$

Soit maintenant $h \in(\mathcal{B}[\log x] \otimes \mathcal{D})^{N=0, \psi=1 \otimes \varphi}$ et $H$ un élément de $\mathcal{B}[\log x] \otimes \mathcal{D}$ tel que $D^{k}(H)=h$ (son existence a été montrée dans le lemme 1.2.9). Les images par 
$D^{k}$ de $N(H)$ et $\left(\psi-p^{-k}(1 \otimes \varphi)\right) H$ sont nulles et donc

$$
\left\{\begin{array}{l}
N(H)=\sum_{j<k} \lambda_{j-k} \frac{\log ^{j}(1+x)}{j !} \stackrel{\text { déf }}{=} \Lambda \\
\left(\psi-p^{-k}(1 \otimes \varphi)\right) H=\sum_{j<k} p^{-j} \mu_{j-k} \frac{\log ^{j}(1+x)}{j !}=\psi(\mathfrak{M})
\end{array}\right.
$$

avec $\mathfrak{M}=\sum_{j<k} \mu_{j-k}\left(\log ^{j}(1+x) / j !\right)$. La relation

$$
\begin{aligned}
(p N \otimes 1+1 \otimes N) & \left(\psi-p^{-k}(1 \otimes \varphi)\right) \\
= & \left(\psi-p^{-k+1}(1 \otimes \varphi)\right) N \otimes 1+\left(\psi-p^{-k+1}(1 \otimes \varphi)\right) 1 \otimes N \\
& =\left(\psi-p^{-k+1}(1 \otimes \varphi)\right)(N \otimes 1+1 \otimes p N) \\
& =\left(\psi-p^{-k+1}(1 \otimes \varphi)\right) N
\end{aligned}
$$

implique que

$$
N \psi(\mathfrak{M})=\left(\psi-p^{-k+1}(1 \otimes \varphi)\right) \Lambda
$$

$(N \otimes 1$ est nul sur $\Lambda$ et $\mathfrak{M})$. On en déduit que

$$
N \mathfrak{M}=\left(1-p^{-k+1} \Phi\right) \Lambda
$$

car $\psi$ est un isomorphisme sur les éléments de $\sum_{i} \log ^{i}(1+x) \mathcal{D}$. Sur les coefficients, cette équation se traduit par les relations

$$
N \mu_{j-k}=\left(1-p^{-k+j+1} \varphi\right) \lambda_{j-k} .
$$

Si l'on change $H$ en $H+\sum_{j<k} \nu_{j-k} \log ^{j}(1+x) / j$ ! (ce qui ne change par $\left.D^{k}(H)\right), \lambda_{j-k}$ est changé en $\lambda_{j-k}+N \nu_{j-k}$ et $\mu_{j-k}$ en $\mu_{j-k}+\left(1-p^{-k+j} \varphi\right) \nu_{j-k}$. De manière plus concise, $\Lambda$ est changé en $\Lambda+N \mathcal{N}$ et $\mathfrak{M}$ en $\mathfrak{M}+(1-\Phi) \mathcal{N}$. Ainsi, la classe de $\left(\lambda_{i}, \mu_{i}\right)$ est bien définie dans $\mathcal{C}(\mathcal{D}[i])$. On définit alors l'application

$$
\widetilde{\mathcal{R}}^{(k)}=\left(\widetilde{\mathcal{R}}_{i}^{(k)}\right)_{-k \leqslant i<0}: \widetilde{\mathcal{D}}_{\infty}(\mathcal{D}) \longrightarrow \oplus_{-k \leqslant i<0} \mathcal{C}(\mathcal{D}[i])
$$

par

$$
\widetilde{\mathcal{R}}_{i}^{(k)}(h)=\text { la classe de }\left(\lambda_{i}, \mu_{i}\right) \text { dans } \mathcal{C}(\mathcal{D}[i]) .
$$

Il est maintenant clair que $h$ appartient à l'image par $D^{k} \operatorname{de}(\mathcal{B}[\log x] \otimes \mathcal{D})^{N=0, \psi=1 \otimes \varphi}$ si et seulement si $\widetilde{\mathcal{R}}^{(k)}(h)=0$. Il est facile de voir que l'action de $G_{\infty}$ est donnée par $\chi^{i}$.

\subsection{Quelques $G_{\infty}$-modules}

2.3.1. Définition. - On définit 1) $\mathcal{D}_{\infty, g}(\mathcal{D})$ comme le sous-module des éléments $g$ de $\mathcal{D}_{\infty}^{\text {gros }}(\mathcal{D})$ tels qu'il existe $r \in \mathbb{Z}$ pour lequel l'équation $\left(1-p^{r} \Phi\right) G=D^{r}(g)$ ait une solution dans $(\mathcal{B}[\log x] \otimes \mathcal{D})^{N=0, \psi=p^{r}(1 \otimes \varphi)}$; 
2) $\mathcal{D}_{\infty, f}(\mathcal{D})$ comme le sous-module des éléments $g$ de $\mathcal{D}_{\infty, g}(\mathcal{D})$ tels qu'il existe une famille $\left(G_{k}\right)_{k \in \mathbb{Z}}$ avec $G_{k} \in(\mathcal{B}[\log x] \otimes \mathcal{D})^{N=0}$ pour tout $k \in \mathbb{Z}, D\left(G_{k}\right)=G_{k+1}$ et un entier $r \in \mathbb{Z}$ tel que $\left(1-p^{r} \Phi\right) G_{r}=D^{r}(g)$;

3) $\mathcal{D}_{\infty, e}(\mathcal{D})$ comme le sous-module des éléments $g$ de $\mathcal{D}_{\infty, g}(\mathcal{D})$ tels que l'équation $\left(1-p^{k} \Phi\right) G=D^{k}(g)$ ait une solution dans $(\mathcal{B}[\log x] \otimes \mathcal{D})^{N=0, \psi=p^{k}(1 \otimes \varphi)}$ pour tout $k \in \mathbb{Z}$;

4) $\mathcal{D}_{\infty, h}(\mathcal{D})$ comme le sous-module des éléments $g$ de $\mathcal{D}_{\infty, g}(\mathcal{D})$ tels qu'il existe une famille $\left(G_{k}\right)_{k \in \mathbb{Z}}$ avec $G_{k} \in(\mathcal{B}[\log x] \otimes \mathcal{D})^{\psi=p^{k}(1 \otimes \varphi)}$ pour tout $k \in \mathbb{Z}, D\left(G_{k}\right)=G_{k+1}$ et un entier $r \in \mathbb{Z}$ tel que $\left(1-p^{r} \Phi\right) G_{r}=D^{r}(g)$.

Il est facile de voir que si $g \in \mathcal{D}_{\infty, e}(\mathcal{D})$, il existe une famille de solutions $G_{k}$ de l'équation $\left(1-p^{k} \Phi\right) G_{k}=D^{k}(g)$ telles que $D\left(G_{k}\right)=G_{k+1}$. Ainsi, on peut aussi définir $\mathcal{D}_{\infty, e}(\mathcal{D})$ comme le sous-module des éléments $g$ de $\mathcal{D}_{\infty, g}(\mathcal{D})$ tels qu'il existe une famille $\left(G_{k}\right)_{k \in \mathbb{Z}}$ avec $G_{k} \in(\mathcal{B}[\log x] \otimes \mathcal{D})^{N=0, \psi=1 \otimes \varphi}$ pour tout $k \in \mathbb{Z}, D\left(G_{k}\right)=G_{k+1}$ et un entier $r \in \mathbb{Z}$ tel que $\left(1-p^{r} \Phi\right) G_{r}=D^{r}(g)$. Il est alors automatique que $\left(1-p^{k} \Phi\right) G_{k}=D^{k}(g)$ pour tout $k \in \mathbb{Z}$ (on le fait par récurrence descendante, comme $\left(1-p^{k} \Phi\right) G_{k}-D^{k}(g)$ est dans le noyau de $\psi$ et de dérivée nulle par hypothèse de récurrence, il est nul).

Les $\mathcal{D}_{\infty, *}(\mathcal{D})$ pour $* \in\{e, f, g\}$ sont des $\mathcal{H}\left(G_{\infty}\right)$-modules et on a

$$
\begin{aligned}
& \mathcal{D}_{\infty, e}(\mathcal{D}) \subset \mathcal{D}_{\infty, f}(\mathcal{D}) \subset \mathcal{D}_{\infty, g}(\mathcal{D}) \subset \mathcal{D}_{\infty}^{\text {gros }}(\mathcal{D}) \\
& \mathcal{D}_{\infty, e}(\mathcal{D}) \subset \mathcal{D}_{\infty, h}(\mathcal{D}) \subset \mathcal{D}_{\infty, g}(\mathcal{D})
\end{aligned}
$$

Les définitions sont fonctorielles en $\mathcal{D}$ et «invariantes par twist » : la dérivation induit un isomorphisme de $\mathcal{H}\left(G_{\infty}\right)$-modules de

$$
D: \mathcal{D}_{\infty, *}(\mathcal{D}) \stackrel{\cong}{\longrightarrow} \mathcal{D}_{\infty, *}(\mathcal{D}[1])(1)
$$

Si $r$ est suffisamment grand pour que $D$ soit une bijection de $\widetilde{\mathcal{D}}_{\infty}(\mathcal{D}[s])$ dans $\widetilde{\mathcal{D}}_{\infty}(\mathcal{D}[s+1])$ pour $s \geqslant r$ et pour $s \leqslant-r$, alors

$$
\begin{aligned}
& \mathcal{D}_{\infty, e}(\mathcal{D})=D^{r}\left(1-p^{-r} \Phi\right) \widetilde{\mathcal{D}}_{\infty}(\mathcal{D}[-r]), \\
& \mathcal{D}_{\infty, g}(\mathcal{D})=D^{-r}\left(1-p^{r} \Phi\right) \widetilde{\mathcal{D}}_{\infty}(\mathcal{D}[r])
\end{aligned}
$$

2.3.2. Construisons une application

$$
\mathcal{R}_{\mathcal{D}}: \mathcal{D}_{\infty, g}(\mathcal{D}) \longrightarrow \oplus_{j \in \mathbb{Z}} \mathcal{C}(\mathcal{D}[j])(j)
$$

de la manière suivante. Soit $g \in \mathcal{D}_{\infty, g}(\mathcal{D})$. Pour $s$ assez grand, l'équation

$$
\left(1-p^{s} \Phi\right) G_{s}=D^{s}(g)
$$

a une solution $G_{s}$ appartenant à $(\mathcal{B}[\log x] \otimes \mathcal{D})^{\psi=p^{s}(1 \otimes \varphi), N=0}$. Notons $\left(G_{k}\right)_{k \in \mathbb{Z}}$ une famille d'éléments $G_{k}$ de $\mathcal{B}[\log x] \otimes \mathcal{D}$ vérifiant $D\left(G_{k}\right)=G_{k+1}$ avec pour $G_{s}$ la solution 
précédente. Posons $G=G_{0}$ et abusivement $D^{-k}(G)=G_{k}$. Alors,

$$
\begin{gathered}
N D^{-r}(G)=\sum_{j \geqslant 0} \lambda_{j-r} \frac{\log ^{j}(1+x)}{j !} \sum_{j \geqslant-r} \lambda_{j} \frac{\log ^{j+r}(1+x)}{(j+r) !} \\
\left(\psi-1 \otimes p^{-r} \varphi\right) D^{-r}(G)=\sum_{0 \leqslant j} \mu_{j-r} \frac{p^{-j} \log ^{j}(1+x)}{j !}=p^{-r} \sum_{j \geqslant-r} \mu_{j} \frac{p^{-j} \log ^{j+r}(1+x)}{(j+r) !}
\end{gathered}
$$

avec les $\lambda_{j}$ et $\mu_{j}$ presque tous nuls. On a $N \mu_{j}=\left(1-p^{-j+1} \varphi\right) \lambda_{j}$. On définit $\mathcal{R}_{\mathcal{D}}(g)$ comme la famille (finie) des classes des couples $\left(\lambda_{j}, \mu_{j}\right)$ dans $\mathcal{C}(\mathcal{D}[j])(j)$. Les $D^{-r}(G)$ ne sont pas définis de manière unique mais l'image de $g$ dans $\mathcal{C}(\mathcal{D}[j])(j)$ ne dépend pas des choix (nous le démontrerons un peu plus loin, $\S 2.3 .2$ ). Notons $\overline{\mathcal{R}}_{\mathcal{D}}$ le composé de $\mathcal{R}_{\mathcal{D}}$ avec la projection sur $\oplus_{i \in \mathbb{Z}}(\mathcal{D} / N \mathcal{D})^{\varphi=p^{-i-1}}(i)$ :

$$
\overline{\mathcal{R}}_{\mathcal{D}}: \mathcal{D}_{\infty, g}(\mathcal{D}) \longrightarrow \oplus_{i \in \mathbb{Z}}(\mathcal{D} / N \mathcal{D})^{\varphi=p^{-i-1}}(i)
$$

On peut récrire ce qui précède de la manière suivante. Soit

$$
\mathcal{U}(\mathcal{D})=\oplus_{j \in \mathbb{Z}} \mathcal{D}[j](j)=\oplus \mathcal{D} \mathbf{t}_{j}
$$

où $\mathbf{t}_{j}$ est une base du $j$-ième facteur. On pose $\varphi \mathbf{t}_{j}=p^{j} \mathbf{t}_{j}, \psi\left(\mathbf{t}_{j}\right)=p^{-j} \mathbf{t}_{j}$, $\tau\left(\mathbf{t}_{j}\right)=\chi^{j}(\tau) \mathbf{t}_{j}, D\left(\mathbf{t}_{j}\right)=\mathbf{t}_{j+1}$. Pour $k \in \mathbb{Z}$, on note $D^{k}: \mathcal{U}(\mathcal{D}) \rightarrow \mathcal{B}$ l'application définie par

$$
D^{k}\left(\sum_{j} b_{j} \mathbf{t}_{j}\right)=\sum_{j \geqslant k} b_{j} \frac{\log ^{j-k}(1+x)}{(j-k) !} .
$$

On vérifie aisément que tous les opérateurs que l'on a nommé $D$ sont compatibles et que l'abus de notation ne porte pas à conséquence : $D^{k+1}=D^{k} \circ D$. De plus, $D^{k}$ est compatible avec les opérateurs $\varphi$ et $\psi$ et l'action de $G_{\infty}: D^{k} \circ \varphi=p^{k} \varphi \circ D^{k}$, $D^{k} \circ \psi=p^{-k} \psi \circ D^{k}, D^{k} \circ \tau=\chi^{k}(\tau) \tau \circ D^{k}$. Ainsi, si $\left(1-p^{s} \Phi\right) G_{s}=D^{s}(g)$ a une solution $G_{s}$ appartenant à $(\mathcal{B}[\log x] \otimes \mathcal{D})^{\psi=p^{s}(1 \otimes \varphi), N=0}$ et si $\left(G_{k}\right)_{k \in \mathbb{Z}}$ est une famille d'éléments $G_{k}$ de $\mathcal{B}[\log x] \otimes \mathcal{D}$ vérifiant $D\left(G_{k}\right)=G_{k+1}$, il existe $\mathfrak{M}$ et $\Lambda$ appartenant à $\mathcal{U}(\mathcal{D})$ tels que

$$
\begin{aligned}
N G_{k} & =D^{k}(\Lambda) \\
(\psi-1 \otimes \varphi) G_{k} & =\psi\left(D^{k}(\mathfrak{M})\right)
\end{aligned}
$$

et si $g=(1-\Phi) G_{0}-D^{0}(\mathfrak{M})$, on a $D^{k}(g)=(1-\Phi) G_{k}-D^{k}(\mathfrak{M})$ pour tout $k \in \mathbb{Z}$.

On considère alors le complexe $\mathfrak{C}_{\infty}(\mathcal{D})$ de longueur 2 :

$$
0 \longrightarrow \mathcal{U}(\mathcal{D}) \stackrel{\delta_{0}}{\longrightarrow} \mathcal{U}(\mathcal{D}) \times \mathcal{U}(\mathcal{D}) \stackrel{\delta_{1}}{\longrightarrow} \mathcal{U}(\mathcal{D}) \longrightarrow 0
$$

avec

$$
\begin{aligned}
\delta_{0}(\nu) & =(N \nu,(1-\Phi) \nu) \\
\delta_{1}(\Lambda, \mathfrak{M}) & =N \mathfrak{M}-(1-p \Phi) \Lambda
\end{aligned}
$$


Posons $\mathcal{C}_{\infty}(\mathcal{D})=H^{1}\left(\mathfrak{C}_{\infty}^{\cdot}(\mathcal{D})\right)$ :

$$
\mathcal{C}_{\infty}(\mathcal{D})=\oplus_{i \in \mathbb{Z}} \mathcal{C}(\mathcal{D}[i])(i)
$$

On a la suite exacte naturelle

$$
0 \longrightarrow \mathcal{U}(\mathcal{D})^{N=0} /(1-\Phi) \mathcal{U}(\mathcal{D})^{N=0} \longrightarrow \mathcal{C}_{\infty}(\mathcal{D}) \longrightarrow(\mathcal{U}(\mathcal{D}) / N \mathcal{U}(\mathcal{D}))^{\Phi=p^{-1}} \longrightarrow 0 .
$$

On peut réinterpréter l'application

$$
\mathcal{R}_{\mathcal{D}}: \mathcal{D}_{\infty, g}(\mathcal{D}) \longrightarrow \oplus_{i \in \mathbb{Z}} \mathcal{C}(\mathcal{D}[i])(i)
$$

comme une application (notée de la même manière)

$$
\mathcal{R}_{\mathcal{D}}: \mathcal{D}_{\infty, g}(\mathcal{D}) \longrightarrow \mathcal{C}_{\infty}(\mathcal{D})
$$

Posons aussi $\mathcal{U}_{0}(\mathcal{D})=\oplus_{j \geqslant 0} \mathcal{D} \log ^{j}(1+x)$ et $\mathcal{C}_{\infty, \geqslant 0}(\mathcal{D})$ le sous-module de $\mathcal{C}_{\infty}(\mathcal{D})$ formé des éléments provenant de $\mathcal{U}_{0}(\mathcal{D})$ :

$$
\mathcal{C}_{\infty, \geqslant 0}(\mathcal{D}) \cong \oplus_{i \geqslant 0} \mathcal{C}(\mathcal{D}[i])(i)
$$

Nous devons quand même vérifier que $\mathcal{R}_{\mathcal{D}}$ ne dépend pas du choix des $D^{r}(G)$, ce qui se déduit du lemme suivant.

2.3.3. Lemme. — Le noyau de $\Phi-1$ sur $\mathcal{B}[\log x] \otimes \mathcal{D}$ est égal à

$$
\oplus_{j \geqslant 0} \mathcal{D}^{\varphi=p^{-j}} \log ^{j}(1+x) \subset \mathcal{U}_{0}(\mathcal{D}) .
$$

D'autre part, l'intersection de $(1-\Phi) \mathcal{B}[\log x] \otimes \mathcal{D}$ et de $\mathcal{U}_{0}(\mathcal{D})$ est égale à $(1-\Phi) \mathcal{U}_{0}(\mathcal{D})$; en particulier, toutes les solutions de l'équation $(1-\Phi) X=u$ avec $u \in \mathcal{U}_{0}(\mathcal{D})$ appartiennent à $\mathcal{U}_{0}(\mathcal{D})$.

Démonstration. - Montrons que si $G \in \mathcal{B}[\log x] \otimes \mathcal{D}$ vérifie $\Phi(G)=G$, pour $k$ assez grand, $D^{k}(G)=0$. En effet, $H=D^{k}(G)$ vérifie $H=p^{k} \Phi(H)$. Le coefficient $H_{0}$ de plus haut degré $r \geqslant 0$ en $\log x$ de $H$ vérifie alors

$$
H_{0}=p^{k+r} \Phi\left(H_{0}\right)
$$

et donc

$$
H_{0}=p^{(k+r) n} \Phi^{n}\left(H_{0}\right) .
$$

On en déduit que pour $\rho>p^{-1 /(p-1)}$ tel que $H_{0} \in \mathcal{H}^{[\rho, 1[}$, on a

$$
\left\|H_{0}\right\|_{\rho^{1 / p^{n}}} \leqslant\left\|p^{n(k+r)}(1 \otimes \varphi)^{n} H_{0}\right\|_{\rho} .
$$

Donc, pour $k$ tel que $\left\|p^{k} \varphi\right\|_{\mathcal{D}}<1$, la limite de $\left\|H_{0}\right\|_{\rho^{1 / p^{n}}}$ lorsque $n \rightarrow \infty$ est nulle. On en déduit la nullité de $H_{0}$ et celle de $H$ (comme $\varphi$ conserve $\mathcal{H}$ et $\mathcal{B}_{-}$, on peut séparer le cas où $H_{0}$ appartient à $\mathcal{B}_{-}$et le cas où il appartient à $\left.\mathcal{H}\right)$.

Donc $G=\sum_{0 \leqslant j<k} a_{j} \log ^{j}(1+x)$. La condition $\Phi(G)=G$ implique alors que $\varphi a_{j}=p^{-j} a_{j}$.

Montrons maintenant que l'intersection de $(1-\Phi) \mathcal{B}[\log x] \otimes \mathcal{D}$ et de $\mathcal{U}_{0}(\mathcal{D})$ est égale à $(1-\Phi) \mathcal{U}_{0}(\mathcal{D})$. Soit $G \in \mathcal{B}[\log x] \otimes \mathcal{D}$ vérifiant l'équation $(1-\Phi) G=u \in \mathcal{U}_{0}(\mathcal{D})$. Il existe un entier $r$ tel que $D^{r}(u)=0$. On a alors $\left(1-p^{r} \Phi\right) D^{r}(G)=0$. On déduit de 
la première partie du lemme appliqué à $\mathcal{D}[r]$ que $D^{r}(G) \in \mathcal{H} \otimes \mathcal{D}$. Donc $G \in \mathcal{H} \otimes \mathcal{D}$. On a alors pour tout entier $r$ l'équation $\left(1-p^{r} \varphi\right)\left(D^{r}(G)(0)\right)=D^{r}(u)(0)$. Comme

$$
u=\sum_{r} D^{r}(u)(0) \frac{\log ^{r}(1+x)}{r !}, \quad v=\sum_{r} D^{r}(G)(0) \frac{\log ^{r}(1+x)}{r !}
$$

est une solution de l'équation $(1-\Phi) v=u$. Toutes les solutions appartiennent donc à $\mathcal{U}_{0}(\mathcal{D})$.

2.3.4. Proposition. - Les suites suivantes de $G_{\infty}$-modules sont exactes :

$$
\begin{gathered}
0 \longrightarrow \mathcal{D}_{\infty, e}(\mathcal{D}) \longrightarrow \mathcal{D}_{\infty, g}(\mathcal{D}) \stackrel{\mathcal{R}_{\mathcal{D}}}{\longrightarrow} \mathcal{C}_{\infty}(\mathcal{D}) \\
0 \longrightarrow \mathcal{D}_{\infty, f}(\mathcal{D}) \longrightarrow \mathcal{D}_{\infty, g}(\mathcal{D}) \stackrel{\overline{\mathcal{R}}_{\mathcal{D}}}{\longrightarrow}\left(\mathcal{U}(\mathcal{D}) / N \mathcal{U}(\mathcal{D})^{\Phi=p^{-1}},\right. \\
0 \longrightarrow \mathcal{D}_{\infty, e}(\mathcal{D}) \longrightarrow \mathcal{D}_{\infty, f}(\mathcal{D}) \stackrel{\mathcal{R}_{\mathcal{D}}}{\longrightarrow} \mathcal{U}(\mathcal{D})^{N=0} /(1-\Phi) \mathcal{U}(\mathcal{D})^{N=0} .
\end{gathered}
$$

Démonstration. - Faisons par exemple la première. Soit $g \in \mathcal{D}_{\infty, g}(\mathcal{D})$ tel que $\mathcal{R}_{\mathcal{D}}(g)=0$. Il existe un entier $s$ et un choix des $G_{r}=D^{r}(G)$ tel que

$$
G_{r} \in(\mathcal{B}[\log x] \otimes \mathcal{D})^{N=0, \psi=p^{r}(1 \otimes \varphi)}
$$

pour $r>s$. Comme $\mathcal{R}_{\mathcal{D}, s}(g)=0, \lambda_{s}=N \nu_{s}, \mu_{s}=\left(1-p^{s} \varphi\right) \nu_{s}$ avec $\nu_{s} \in \mathcal{D}$. Alors, $\widetilde{\mathbb{G}}_{s}=G_{s}-\nu_{s}$ appartient à $\left.\mathcal{B}[\log x] \otimes \mathcal{D}\right)^{\psi=p^{s}(1 \otimes \varphi), N=0}$ et vérifie $D\left(G_{s}\right)=G_{s+1}$, $(1-\Phi) G_{s}=D^{s}\left(g_{s}\right)$. La réciproque est facile.

\subsection{Exemple : $N=0$}

Nous allons étudier le cas particulier où $\mathcal{D}$ est un $\varphi$-module avec action triviale de $N$ et faire le lien avec des espaces introduits dans des articles antérieurs. Il est démontré dans [19] la suite exacte

$$
0 \longrightarrow(1-\Phi)(\mathcal{H} \otimes \mathcal{D})^{\psi=1 \otimes \varphi} \longrightarrow \mathcal{H}^{\psi=0} \otimes \mathcal{D} \stackrel{\left(\Delta_{i}\right)_{i} \geqslant 0}{\longrightarrow} \underset{i \geqslant 0}{\oplus}\left(\mathcal{D} /\left(1-p^{i} \varphi\right) \mathcal{D}\right)(i) \longrightarrow 0
$$

L'application $\Delta_{i}: \mathcal{H}^{\psi=0} \otimes \mathcal{D} \rightarrow\left(\mathcal{D} /\left(1-p^{i} \varphi\right) \mathcal{D}\right)(i)$ (avec un petit changement de notations) est donnée par

$$
\Delta_{i}(f) \equiv D^{i}(f)(0) \bmod \left(1-p^{i} \varphi\right) \mathcal{D}
$$

et a un sens pour $i<0$ car la dérivation est un isomorphisme sur $\mathcal{H}^{\psi=0}$.

2.4.1. Proposition. - Soit $\mathcal{D}$ un $\varphi$-module de dimension finie.

1) $\mathcal{D}_{\infty, g}(\mathcal{D})$ est contenu dans $\mathcal{B} \otimes \mathcal{D}$ et si $g \in \mathcal{D}_{\infty, g}(\mathcal{D})$, on a

$$
g \equiv \sum_{i} \overline{\mathcal{R}}_{\mathcal{D}, i}(g) \mathfrak{L}_{-i}^{(p)} \bmod \mathcal{H}^{\psi=0} \otimes \mathcal{D}
$$

avec $\mathfrak{L}_{-i}^{(p)}=D^{i}\left(\mathfrak{L}_{0}^{(p)}\right)$ 
2) On a

$$
\mathcal{D}_{\infty, f}(\mathcal{D})=\mathcal{H}^{\psi=0} \otimes \mathcal{D}
$$

et l'application $\mathcal{R}_{\mathcal{D}}$ coïncide sur $\mathcal{D}_{\infty, f}(\mathcal{D})$ avec $\Delta=\oplus_{i \in \mathbb{Z}} \Delta_{i}$.

3) En particulier, $\mathcal{D}_{\infty, e}(\mathcal{D})$ est égal au noyau de $\Delta$.

Ici, $\overline{\mathcal{R}}_{\mathcal{D}, i}$ est bien sûr la $i$-ième composante de $\overline{\mathcal{R}}_{\mathcal{D}}$ :

$$
\overline{\mathcal{R}}_{\mathcal{D}, i}: \mathcal{D}_{\infty, g}(\mathcal{D}) \longrightarrow \mathcal{D}^{\varphi=p^{-i-1}}(i)
$$

Ainsi, $\mathcal{D}_{\infty, f}(\mathcal{D})$ est un $\mathcal{H}\left(G_{\infty}\right)$-module libre de type fini de rang $\operatorname{dim}_{\mathbb{Q}_{p}} \mathcal{D}$. Il en est de même de $\mathcal{D}_{\infty, e}(\mathcal{D})$ car en fait, $\mathcal{D}_{\infty, e}(\mathcal{D})$ est obtenu comme le tensorisé avec $\mathcal{H}\left(G_{\infty}\right)$ du $\mathbb{Q}_{p} \otimes \mathbb{Z}_{p} \llbracket G_{\infty} \rrbracket$-module noyau de

$$
\Delta: \mathbb{Z}_{p} \llbracket x \rrbracket^{\psi=0} \otimes \mathcal{D} \rightarrow \oplus_{i}\left(\mathcal{D} /\left(1-p^{i} \varphi\right) \mathcal{D}\right)(i) .
$$

Nous montrons la proposition précédente jusqu'à la fin du paragraphe 2.4. Commençons par le cas particulier $\mathcal{D}=\mathbb{Q}[s]$.

2.4.2. Lemme. - Si s est un entier négatif, le noyau de $\psi-p^{s}$ dans $\mathcal{B}$ est contenu dans $\mathcal{H}$. Si s est un entier positif, le noyau de $\psi-p^{s}$ dans $\mathcal{B}$ est contenu dans $x^{-s-1} \mathcal{H}$, son intersection avec $\mathcal{B}_{-}$est un $\mathbb{Q}_{p}$-espace vectoriel de dimension 1 engendré par $D^{s}\left(x^{-1}\right)$ et on a la suite exacte de $G_{\infty}$-modules

$$
0 \longrightarrow \mathcal{H}^{\psi=p^{s}} \longrightarrow \mathcal{B}^{\psi=p^{s}} \longrightarrow \mathbb{Q}_{p}(-s-1) \longrightarrow 0 .
$$

Démonstration. - Comme $\psi$ conserve $\mathcal{H}$ et $\mathcal{B}_{-}$, il suffit de calculer le noyau de $\psi-p^{s}$ sur $\mathcal{B}_{-}$. Soit $F \in \mathcal{B}_{-}$tel que $\psi(F)=p^{s} F$. Fixons un réel $\rho$ tel que $F \in \mathcal{H}^{[\rho, \infty]}$. Supposons d'abord $s<0$. On a alors

$$
p^{-s}\|F\|_{\rho}=\|\psi(F)\|_{\rho} \leqslant\|F\|_{\rho},
$$

ce qui implique la nullité de $F$. Supposons maintenant $s \geqslant 0$. Prenons $s=0$ et $\psi(F)=F$ avec $F=\sum_{r>0} a_{r} x^{-r} \in \mathcal{H}_{0}^{[\rho, \infty)}$. Comme $\psi(1 / x)=1 / x$, on peut supposer en ajoutant à $F$ un multiple de $1 / x$ que $a_{1}=0$. Si $F$ est non nul, on peut supposer que tous les coefficients de $F$ ne sont pas divisibles par $p$. Soit alors $r_{0}$ le plus grand entier tel que $a_{r_{0}}$ n'est pas divisible par $p$. Comme $\psi\left(1 / x^{r_{0}}\right)-p^{r_{0}-1} / x^{r_{0}} \in\left(1 / x^{r_{0}-1}\right) \mathbb{Z}_{p} \llbracket x \rrbracket$, on a

$$
\psi(F)-F=a_{r_{0}} \frac{p^{r_{0}-1}-1}{x^{r_{0}}}+p h+\sum_{k<r_{0}} \frac{b_{r}}{x^{r}}
$$

avec $h \in \mathbb{Z}_{p} \llbracket 1 / x \rrbracket$. Comme $a_{r_{0}}$ est une unité, on en déduit que $r_{0}=1$, ce qui est contradictoire. Donc $F$ est un multiple de $x^{-1}$. Passons à $s>0$ et soit $F \in \mathcal{B}_{-}$tel que $\psi(F)=p^{s} F$. Remarquons que l'équation $\psi(F)=p^{s} F$ implique que $F \in \mathcal{H}^{[\rho, 1)}$ pour un réel $\rho<p^{-1 /(p-1)}$ : en effet, si $F \in \mathcal{H}^{[\eta, 1)}$ avec $\eta>p^{-1 /(p-1)}, \psi(F)$ et donc 
$F$ appartiennent à $\mathcal{H}^{\left[\eta^{p}, 1\right)}$. En recommençant, on obtient un $\eta<p^{-1 /(p-1)}$. D'autre part, on a

$$
\begin{aligned}
\psi\left(F \log ^{s}(1+x)\right) & =p^{-s} \psi\left(F \varphi \log ^{s}(1+x)\right)=p^{-s} \psi(F) \log ^{s}(1+x) \\
& =F \log ^{s}(1+x)
\end{aligned}
$$

Ainsi, $G=F \log ^{s}(1+x)$ est de la forme $x^{-1} U$ avec $U \in \mathcal{H}$. En multipliant par $x$ on en déduit que

$$
x \log ^{s}(1+x) F=U .
$$

Cela implique que $U\left(\zeta_{n}-1\right)$ est nul pour $n \geqslant 1$ et donc que $U$ est divisible par $\log (1+x) / x$. En itérant, on en déduit que $U$ est divisible par $(\log (1+x) / x)^{s}$ dans $\mathcal{H}$, d'où $x^{s+1} F \in \mathcal{H}$. Ce qui donne la première partie du lemme. Il est clair qu'alors l'intersection du noyau de $\psi-p^{s}$ avec $\mathcal{B}_{\text {- }}$ est un $\mathbb{Q}_{p}$-espace vectoriel de dimension finie. Montrons qu'il est de dimension 1. Les $D^{j}\left(x^{-1}\right)$ forment une base des polynômes en $x^{-1}$. On peut donc écrire : $F=\sum_{j=0}^{r} a_{j} D^{j}\left(x^{-1}\right)$. Comme $\psi\left(D^{j}\left(x^{-1}\right)\right)=p^{j} D^{j}\left(x^{-1}\right)$, on déduit de l'équation $\psi(F)=p^{s} F$ que $a_{j}$ est nul sauf pour $j=s$. Ainsi, $\mathcal{B}_{-} \cap \operatorname{ker}\left(\psi-p^{s}\right)$ est le $\mathbb{Q}_{p}$-espace vectoriel engendré par $D^{s}\left(x^{-1}\right)$.

Démontrons la compatibilité de la suite exacte avec l'action de $G_{\infty}$. On a pour $\tau \in G_{\infty}$

$$
\tau\left(D^{s}\left(x^{-1}\right)\right)=\chi(\tau)^{-s} D^{s}\left(\tau\left(x^{-1}\right)\right)
$$

Donc

$$
\left(\chi(\tau)^{s+1} \tau-1\right) D^{s}\left(x^{-1}\right)=D^{s}\left(\frac{\chi(\tau)}{(1+x)^{\chi(\tau)}-1}-\frac{1}{x}\right) \in \mathbb{Z}_{p} \llbracket x \rrbracket .
$$

Donc la suite exacte de la proposition n'est pas scindée en tant que suite exacte de $G_{\infty}$-modules et l'action de $G_{\infty}$ sur le quotient est donnée par $\chi^{-s-1}$.

2.4.3. Proposition. - Soit $\mathcal{D}$ un $\varphi$-module. Il existe un entier $r \geqslant 0$ tel que $(\mathcal{B} \otimes \mathcal{D})^{\psi=1 \otimes \varphi}$ soit contenu dans $x^{-r} \mathcal{H} \otimes \mathcal{D}$ et on a une suite exacte de $G_{\infty}$-modules

$$
0 \longrightarrow(\mathcal{H} \otimes \mathcal{D})^{\psi=1 \otimes \varphi} \longrightarrow(\mathcal{B} \otimes \mathcal{D})^{\psi=1 \otimes \varphi} \longrightarrow \oplus_{j<0} \mathcal{D}^{\varphi=p^{-j-1}}(j) \longrightarrow 0
$$

L'application $\widetilde{\partial}_{j}:(\mathcal{B} \otimes \mathcal{D})^{\psi=1 \otimes \varphi} \rightarrow \mathcal{D}^{\varphi=p^{-j-1}}(j)$ est définie de la manière suivante : si $G \in(\mathcal{B} \otimes \mathcal{D})^{\psi=1 \otimes \varphi}$, il existe des éléments uniques $a_{i}$ de $\mathcal{D}$ pour $i \geqslant 0$, presque tous nuls, tels que

$$
G-\sum_{i \geqslant 1} a_{-i} D^{i-1}(1 / x) \in \mathcal{H} \otimes \mathcal{D}
$$

La condition $\psi(G)=(1 \otimes \varphi) G$ implique que $a_{-i} \in \mathcal{D}^{\varphi=p^{i-1}}$ et on pose $\widetilde{\partial}_{j}(G)=a_{j}$ pour $j<0$. On a ainsi

$$
G-\sum_{j<0} \widetilde{\partial}_{j}(G) D^{-j-1}(1 / x) \in(\mathcal{H} \otimes \mathcal{D})^{\psi=1 \otimes \varphi}
$$

ou encore

$$
G-\sum_{j<0} \widetilde{\partial}_{j}(G) D^{-j}(\log x) \in(\mathcal{H} \otimes \mathcal{D})^{\psi=1 \otimes \varphi}
$$


Démonstration. - La démonstration suit celle du cas particulier où $\mathcal{D}=\mathbb{Q}_{p}[s]$. Soit $\mathcal{D}_{[n / q]}$ une composante isotypique du $\varphi$-module $\mathcal{D}$ avec $q$ et $n$ des entiers premiers entre eux et $q>0$ : il existe un sous- $\mathbb{Z}_{p}$-module $M$ de $\mathcal{D}_{[q / n]}$ de rang maximal et tel que $p^{-n} \varphi^{q}$ soit un automorphisme $u$. Si $F \in \mathcal{H}_{0}^{[\rho, \infty)} \otimes M$ tel que $F$ ne soit pas divisible par $p$, il existe un entier $r$ tel que

$$
F-p F_{1}+\frac{A_{r}}{x^{r}} \in \frac{1}{x^{r-1}} \mathbb{Z}_{p}[x] \otimes M
$$

avec $F_{1} \in \mathcal{H}_{0}^{[\rho, \infty)} \otimes M, A_{r} \in M-p M$. Si $\psi(F)=(1 \otimes \varphi) F$, on a aussi

$$
\psi^{q}(F)=\left(1 \otimes \varphi^{q}\right) F=p^{n}(1 \otimes u) F
$$

et on en déduit que

$$
\left(p^{n}(1 \otimes u)-p^{q(r-1)}\right) A_{r} \in p M
$$

Lorsque $n<0$, comme $A_{r}$ n'est pas divisible par $p$ et que $F \in \mathcal{B}_{-} \otimes \mathcal{D}$, on obtient une contradiction donc $F=0$ ou appartient à $\mathcal{H} \otimes \mathcal{D}$. Lorsque $n=0$ et $q=1$, cela implique que $r=1$ et $F-(1 / x) \delta_{0}(F) \in \mathcal{H} \otimes \mathcal{D}$. Lorsque $n>0$, on procède comme dans la démonstration du lemme 2.4.2. En multipliant par $\log ^{s}(1+x)$ pour $s$ assez grand, on en déduit que $F \in x^{-s-1} \mathcal{H}$. On termine comme dans le lemme précédent.

2.4.4. Remarques. - $\mathrm{Si}\left\|\varphi^{-1}\right\|<1$, on a $\mathcal{D}^{\varphi=p^{s}}=0$ pour tout entier $s \geqslant 0$ et donc $(\mathcal{B} \otimes \mathcal{D})^{\psi=1 \otimes \varphi}=(\mathcal{H} \otimes \mathcal{D})^{\psi=1 \otimes \varphi}$.

Si $g \in(1-\Phi)(\mathcal{B} \otimes \mathcal{D})^{\psi=1 \otimes \varphi}$, définissons $\partial_{j}(g)$ par la condition

$$
g-\sum_{j<0} \partial_{j}(g) D^{-j-1}\left(\mathfrak{L}_{0}^{(p)}\right) \in \mathcal{H}^{\psi=0} \otimes \mathcal{D} .
$$

On a donc $\partial_{j}((1-\Phi) G)=\widetilde{\partial}_{j}(G)$. Comme le noyau de $\Phi-p^{k} \operatorname{sur}(\mathcal{B} \otimes \mathcal{D})^{\psi=1 \otimes \varphi}$ est contenu dans $(\mathcal{H} \otimes \mathcal{D})^{\psi=1 \otimes \varphi}$, on déduit de la suite exacte (2.4.2) la suite exacte

$$
\begin{aligned}
& 0 \longrightarrow(1-\Phi)(\mathcal{H} \otimes \mathcal{D})^{\psi=1 \otimes \varphi} \longrightarrow(1-\Phi)(\mathcal{B} \otimes \mathcal{D})^{\psi=1 \otimes \varphi} \\
& \stackrel{\left(\partial_{j}\right)}{\longrightarrow} \underset{j<0}{\oplus} \mathcal{D}^{\varphi=p^{-j-1}}(j) \longrightarrow 0
\end{aligned}
$$

Montrons maintenant la proposition 2.4.1. On a pour $r$ assez grand,

$$
\mathcal{D}_{\infty, g}(\mathcal{D})=D^{-r}\left(1-p^{r} \Phi\right)(\mathcal{B} \otimes \mathcal{D})^{\psi=1 \otimes p^{r} \varphi} .
$$

Pour simplifier, supposons que $r=0$ (ce qui revient à tordre $\mathcal{D}$, les définitions sont de toute façon faites pour être invariantes par twist). Montrons que $\partial_{j}$ coïncide avec l'application $\overline{\mathcal{R}}_{\mathcal{D}, j}$. Soit $G \in(\mathcal{B} \otimes \mathcal{D})^{\psi=1 \otimes \varphi}$ et $g=(1-\Phi) G$. On a

$$
G-\sum_{j<0} \widetilde{\partial}_{j}(G) D^{-j-1}(1 / x) \in \mathcal{H} \otimes \mathcal{D}
$$


avec $\widetilde{\partial}_{j}(G) \in \mathcal{D}^{\varphi=p^{-j-1}}$. Comme $D$ est surjectif sur $\mathcal{H}$ et que l'image réciproque de $\mathcal{H}$ est contenue dans $\mathcal{H}$, on a

$$
D^{-k}(G) \equiv \sum_{j<0} \widetilde{\partial}_{j}(G) D^{-j-k-1}(1 / x) \bmod \mathcal{H} \otimes \mathcal{D} .
$$

Pour $j<0$, on prend $D^{j}(1 / x)=\mathfrak{L}_{-j}$ et on a

$$
N D^{j}(1 / x)=\frac{\log ^{-j-1}(1+x)}{(-j-1) !} .
$$

Donc

$$
\begin{aligned}
N D^{-k}(G) & =\sum_{j<0} \widetilde{\partial}_{j}(G) \frac{\log ^{k+j}(1+x)}{(k+j) !} \\
& =\sum_{0 \leqslant i<k} \widetilde{\partial}_{i-k}(G) \frac{\log ^{i}(1+x)}{i !} .
\end{aligned}
$$

Donc $\overline{\mathcal{R}}_{\mathcal{D}, i}(g)=\widetilde{\partial}_{i}(G)=\partial_{i}(g)$. Cela démontre la première assertion de la proposition 2.4.1. On en déduit que le noyau de $\overline{\mathcal{R}}_{\mathcal{D}}$ est $\mathcal{H}^{\psi=0} \otimes \mathcal{D}$. Comme c'est aussi par définition $\mathcal{D}_{\infty, f}(\mathcal{D})$, on obtient que

$$
\mathcal{D}_{\infty, f}(\mathcal{D})=\mathcal{H}^{\psi=0} \otimes \mathcal{D} .
$$

Montrons maintenant que les applications $\Delta_{i}: \mathcal{H}^{\psi=0} \otimes \mathcal{D} \rightarrow \mathcal{D} /\left(1-p^{i} \varphi\right) \mathcal{D}$ coïncident avec la restriction de $\mathcal{R}_{\mathcal{D}, i}$ à $\mathcal{D}_{\infty, f}(\mathcal{D})$. On a pour $r$ assez grand

$$
\mathcal{D}_{\infty, e}(\mathcal{D})=D^{-r}\left(1-p^{-r} \Phi\right)(\mathcal{B} \otimes \mathcal{D})^{\psi=1 \otimes p^{-r} \varphi} .
$$

Supposons de nouveau que $r=0$ (attention on ne tord pas dans la même direction). Soit $g \in \mathcal{H}^{\psi=0} \otimes \mathcal{D}: g=(1-\Phi) G$ avec $G \in(\mathcal{H} \otimes \mathcal{D})^{\psi=1 \otimes \varphi}$ et

$$
D^{-k}(g)-\sum_{i \geqslant 0} \mathcal{R}_{\mathcal{D}, i-k}(g) \frac{\log ^{i}(1+x)}{i !} \in\left(1-p^{-k} \Phi\right)(\mathcal{H} \otimes \mathcal{D}) .
$$

En appliquant $D^{i}$ et en prenant la valeur en 0 ,

$$
D^{i}\left(D^{-k}(g)\right)(0) \equiv \mathcal{R}_{\mathcal{D}, i-k}(g) \bmod \left(1-p^{i-k} \varphi\right) \mathcal{D} .
$$

Donc,

$$
\begin{aligned}
\mathcal{R}_{\mathcal{D}, i-k}(g) & \equiv \Delta_{i}\left(D^{-k}(g)\right) \bmod \left(1-p^{i-k} \varphi\right) \mathcal{D} \\
& \equiv \Delta_{i-k}(g) \bmod \left(1-p^{i-k} \varphi\right) \mathcal{D} .
\end{aligned}
$$

On en déduit que le noyau de $\Delta$ est exactement $\mathcal{D}_{\infty, e}(\mathcal{D})$. La proposition 2.4.1 est montrée.

On pose

$$
\begin{aligned}
\mathcal{D}_{\infty, g}^{2}(\mathcal{D}) & =H^{2}(\mathcal{C} \cdot(\mathcal{U}(\mathcal{D}))=\mathcal{U}(\mathcal{D}) /(1-p \Phi, N) \mathcal{U}(\mathcal{D}) \\
& =\oplus_{i \in \mathbb{Z}} H^{2}(\mathfrak{C} \cdot(\mathcal{D}[i]))(i)=\oplus_{i \in \mathbb{Z}}\left(\mathcal{D} /\left(1-p^{i+1} \varphi\right) \mathcal{D}\right)(i)
\end{aligned}
$$


La proposition suivante est maintenant claire :

2.4.5. Proposition. - Soit $\mathcal{D}$ un $\varphi$-module de dimension finie.

1) Les suites de $\mathcal{H}\left(G_{\infty}\right)$-modules

$$
0 \longrightarrow \mathcal{D}_{\infty, f}(\mathcal{D}) \longrightarrow \mathcal{D}_{\infty, g}(\mathcal{D}) \longrightarrow \oplus_{j \in \mathbb{Z}} \mathcal{D}^{\varphi=p^{j}}(-j-1) \longrightarrow 0
$$

et

$$
0 \longrightarrow \mathcal{D}_{\infty, e}(\mathcal{D}) \longrightarrow \mathcal{D}_{\infty, f}(\mathcal{D}) \longrightarrow \oplus_{i \in \mathbb{Z}}\left(\mathcal{D} /\left(1-p^{i} \varphi\right) \mathcal{D}\right)(i) \longrightarrow 0
$$

sont exactes.

2) Si $0 \rightarrow D_{1} \rightarrow D \rightarrow D_{2} \rightarrow 0$ est une suite exacte de $\varphi$-modules, on a les suites naturelles exactes de $\mathcal{H}\left(G_{\infty}\right)$-modules

$$
0 \longrightarrow \mathcal{D}_{\infty, f}\left(\mathcal{D}_{1}\right) \longrightarrow \mathcal{D}_{\infty, f}(\mathcal{D}) \longrightarrow \mathcal{D}_{\infty, f}\left(\mathcal{D}_{2}\right) \longrightarrow 0
$$

et

$$
\begin{aligned}
0 \longrightarrow \mathcal{D}_{\infty, g}\left(\mathcal{D}_{1}\right) \longrightarrow \mathcal{D}_{\infty, g}(\mathcal{D}) & \longrightarrow \mathcal{D}_{\infty, g}\left(\mathcal{D}_{2}\right) \\
& \longrightarrow \mathcal{D}_{\infty, g}^{2}\left(\mathcal{D}_{1}\right) \longrightarrow \mathcal{D}_{\infty, g}^{2}(\mathcal{D}) \longrightarrow \mathcal{D}_{\infty, g}^{2}\left(\mathcal{D}_{2}\right) \longrightarrow 0
\end{aligned}
$$

Si de plus $0 \rightarrow D_{1}^{\varphi=p^{j}} \rightarrow D^{\varphi=p^{j}} \rightarrow D_{2}^{\varphi=p^{j}} \rightarrow 0$ est exacte pour tout entier $j \in \mathbb{Z}$ (on dira que l'extension est e pour tout $j \in \mathbb{Z}$ ), la suite naturelle

$$
0 \longrightarrow \mathcal{D}_{\infty, e}\left(\mathcal{D}_{1}\right) \longrightarrow \mathcal{D}_{\infty, e}(\mathcal{D}) \longrightarrow \mathcal{D}_{\infty, e}\left(\mathcal{D}_{2}\right) \longrightarrow 0
$$

est exacte.

2.4.6. Remarques. - Le scindage $\mathcal{D}_{\infty, g}(\mathcal{D}) \rightarrow \oplus_{j \in \mathbb{Z}} \mathcal{D}^{\varphi=p^{j}}(-j-1)$ explicité au cours de la démonstration

$$
\left(a_{i}\right)_{i \in \mathbb{Z}} \longmapsto \sum_{i \in \mathbb{Z}} a_{i} \mathfrak{L}_{-i}^{(p)}
$$

est l'unique scindage à valeurs dans $\mathcal{B}_{-}^{\psi=0} \otimes \mathcal{D} \cap \mathcal{D}_{\infty, g}(\mathcal{D})$. En effet, $\mathcal{D}_{\infty, f}(\mathcal{D})$ est contenu dans $\mathcal{H}^{\psi=0} \otimes \mathcal{D}$. Autrement dit, c'est l'unique scindage qui s'étend de manière analytique à $|x|>p^{-1 /(p-1)}$ (cf. appendice A). Il a été utilisé dans le cas particulier du carré symétrique d'une représentation $p$-adique de dimension 2 dans $[\mathbf{2 4}]$. Une fois le lien avec la cohomologie galoisienne faite, ce scindage peut être vu comme un moyen de constructions de «points géométriques » dans la cohomologie galoisienne.

\subsection{Exemples de dimension $\leqslant 2$.}

2.5.1. Prenons $\mathcal{D}=\mathbb{Q}_{p}$. Il est facile de construire un élément de $\mathcal{D}_{\infty, g}\left(\mathbb{Q}_{p}\right)$ qui n'est pas dans $\mathcal{D}_{\infty, e}\left(\mathbb{Q}_{p}\right)$ : il s'agit simplement de

$$
\mathfrak{L}_{0}^{(p)}=\frac{1}{x}-\frac{1}{(1+x)^{p}-1}=(1-\varphi) \frac{1}{x} .
$$


Comme $x^{-1}$ est un élément de $(\mathcal{B} \otimes \mathcal{D})^{\psi=1 \otimes \varphi}=\mathcal{B}^{\psi=1}, \mathfrak{L}_{0}^{(p)}$ appartient à $\mathcal{D}_{\infty, g}\left(\mathbb{Q}_{p}\right)$. Par contre, il n'appartient pas à $\mathcal{D}_{\infty, e}\left(\mathbb{Q}_{p}\right)$, ni même à $\mathcal{D}_{\infty, f}\left(\mathbb{Q}_{p}\right)$, car

$$
D^{-k}\left(\mathfrak{L}_{0}^{(p)}\right)=\mathfrak{L}_{k}^{(p)}=\left(1-p^{-k} \varphi\right)\left(\mathfrak{L}_{k}\right)
$$

mais $\mathfrak{L}_{k}$ n'appartient pas à $\mathcal{B}[\log x]^{N=0}$.

2.5.2. Prenons $\mathcal{D}$ de dimension 2 de base $\left(e, e_{1}\right)$ avec $e_{1}=N e, \varphi e=e$. On a donc $\varphi N e=p^{-1} N e$ et $N^{2} e=0$. Si $k$ est un entier positif, soit

$$
L_{k}=\mathfrak{L}_{k} N e-N \mathfrak{L}_{k} e=\mathfrak{L}_{k} N e-\frac{1}{(k-1) !} \log ^{k-1}(1+x) e .
$$

On a

On en déduit que

$$
\left\{\begin{array}{l}
\left(1-p^{-k+1} \Phi\right) L_{k}=\mathfrak{L}_{k}^{(p)} N e \in\left(\mathcal{B}^{\psi=0} \otimes \mathcal{D}\right)^{N=0} \\
L_{k} \in(\mathcal{B}[\log x] \otimes \mathcal{D})^{N=0, \psi=1 \otimes p^{1-k} \varphi} \\
D\left(L_{k}\right)=L_{k-1} .
\end{array}\right.
$$

$$
\mathfrak{L}_{1}^{(p)} N e=\left(1-p^{-1} \varphi\right) \log x \cdot N e=a_{\varphi} \cdot N e
$$

est un élément de $\mathcal{D}_{\infty, e}(\mathcal{D})$. Mais il n'appartient pas à $\mathcal{D}_{\infty, e}\left(\mathcal{D}_{1}\right)$ où $\mathcal{D}_{1}$ est le sous$(\varphi, N)$-module de $\mathcal{D}$ engendré par $N e$ d'après le paragraphe précédent.

Montrons maintenant qu'il existe un élément de $\mathcal{D}_{\infty, g}(\mathcal{D})$ de la forme $(1-\Phi) \mathcal{M}$ avec

$$
\mathcal{M}=\frac{1}{x} e-\left(\frac{\log x}{x}+V_{0}\right) N e \in(\mathcal{B}[\log x] \otimes \mathcal{D})^{N=0, \psi=1 \otimes \varphi}
$$

vérifiant $\overline{\mathcal{R}}_{\mathcal{D},-1}(\mathcal{M}) \equiv e \bmod N \mathcal{D}$. La condition $N \mathcal{M}=0$ est équivalente à ce que $V_{0}$ appartienne à $\mathcal{B}$. La condition $\psi(\mathcal{M})=(1 \otimes \varphi) \mathcal{M}$ se traduit par l'équation

$$
\begin{aligned}
\left(\psi-p^{-1}\right) V_{0} & =-\left(\psi-p^{-1}\right)\left(\frac{\log x}{x}\right) \\
& =-\psi\left(\frac{p^{-1} \varphi \log x-a_{\varphi}}{x}\right)+p^{-1} \frac{\log x}{x} \\
& =\psi\left(\frac{a_{\varphi}}{x}\right) \in \mathcal{B}_{-} .
\end{aligned}
$$

Comme $\|\psi(g)\|_{\rho} \leqslant\|g\|_{\rho}$ pour $g \in \mathcal{H}^{[\rho, \infty]}$, la série $\sum_{n=0}^{\infty} p^{n} \psi^{n}(g)$ converge dans $\mathcal{B}_{-}$ pour un tel $g$ et en particulier pour $g=\psi\left(a_{\varphi} / x\right)$. D'où l'existence de $V_{0}$. Soit $\mathcal{M}_{-1}$ tel que $D\left(\mathcal{M}_{-1}\right)=\mathcal{M}$. Il est facile de voir que

$$
\mathcal{M}_{1}=(\log x-\log (x+1)) e \bmod \mathcal{B}[\log x] \otimes N \mathcal{D} .
$$

et donc que $N\left(\mathcal{M}_{1}\right) \equiv e \bmod \mathcal{B}[\log x] \otimes N \mathcal{D}$. Par définition de $\overline{\mathcal{R}}_{\mathcal{D},-1}$, on a donc

$$
\overline{\mathcal{R}}_{\mathcal{D},-1}((1-\Phi) \mathcal{M}) \equiv e \bmod N \mathcal{D} \text {. }
$$

On en déduit que l'application

$$
\mathcal{D}_{\infty, g}(\mathcal{D}) \longrightarrow(\mathcal{D} / N \mathcal{D})^{\varphi=1}(-1)
$$


est surjective. Comme $(\mathcal{D} / N \mathcal{D})^{\varphi=p^{i}}$ est nul pour tout $i \neq 0$, on en déduit la suite exacte

$$
\begin{gathered}
0 \longrightarrow \mathcal{D}_{\infty, f}(\mathcal{D}) \longrightarrow \mathcal{D}_{\infty, g}(\mathcal{D}) \stackrel{\overline{\mathcal{R}}_{\mathcal{D},-1}}{\longrightarrow} \\
(\mathcal{D} / N \mathcal{D})^{\varphi=1}(-1) \longrightarrow 0 \\
\mathbb{Q}_{p}(-1)
\end{gathered}
$$

D'autre part, $\mathcal{D}^{N=0} /\left(1-p^{j} \varphi\right) \mathcal{D}^{N=0}$ est non nul si et seulement si $j=1$ et est alors égal à $\mathbb{Q}_{p} N e$. D'où la suite exacte

$$
0 \longrightarrow \mathcal{D}_{\infty, e}(\mathcal{D}) \longrightarrow \mathcal{D}_{\infty, f}(\mathcal{D}) \stackrel{\mathcal{R}_{\mathcal{D}, 1}}{\longrightarrow}\left(\mathcal{D}^{N=0} /(1-p \varphi) \mathcal{D}^{N=0}\right)(1) \longrightarrow 0
$$

Par exemple, $(1+x) N e$ est un élément de $\mathcal{D}_{\infty, f}\left(\mathcal{D}^{N=0}\right) \subset \mathcal{D}_{\infty, f}\left(\mathcal{D}^{N=0}\right)$ tel que $\mathcal{R}_{\mathcal{D}, 1}((1+x) N e)=N e$. Les éléments $(1+x) N e$ et $\mathbf{m}=(1-\Phi) \mathcal{M}\left(\right.$ resp. $\mathfrak{L}_{1}^{(p)} N e$ et $\mathbf{m}$ ) forment un système libre de $\mathcal{D}_{\infty, g}(\mathcal{D})$ sur $\mathcal{H}\left(G_{\infty}\right)$. En effet,

$$
\mathbf{m} \equiv \mathfrak{L}_{0}^{(p)} e \bmod \mathcal{B}[\log x] N e
$$

et il n'existe pas de $f \in \mathcal{H}\left(G_{\infty}\right)$ tel que $f \cdot \mathfrak{L}_{0}^{(p)}=0$. Par contre, $(1+x) N e$ et $\mathfrak{L}_{1}^{(p)} N e$ sont liés car $(\chi(\gamma) \gamma-1) \mathfrak{L}_{1}^{(p)} \in \mathbb{Z}_{p} \llbracket G_{\infty} \rrbracket \cdot(1+x)$ pour $\gamma$ un générateur topologique de $\Gamma$.

Nous allons généraliser ces calculs dans le paragraphe suivant. 



\section{CHAPITRE 3}

\section{CONSTRUCTION D'ÉLÉMENTS DE $\mathcal{D}_{\infty, *}(\mathcal{D})$}

Le but de ce paragraphe est de construire des éléments de $\mathcal{D}_{\infty, *}(\mathcal{D})$ pour $* \in\{e, f, g\}$ à partir d'éléments de $\mathcal{D}_{\infty, *}(\overline{\mathcal{D}})$ où $\overline{\mathcal{D}}$ est le $\varphi$-module $\mathcal{D}$ muni de l'opérateur de monodromie nul. Bien que le cas de la construction d'éléments de $\mathcal{D}_{\infty, g}(\mathcal{D})$ contienne le cas de la construction d'éléments de $\mathcal{D}_{\infty, e}(\mathcal{D})$, nous avons préféré traiter indépendamment le cas plus simple, celui étant suffisant pour les $(\varphi, N)$-modules tels que $\varphi$ n'a pas de valeurs propres du type $p^{j}$ avec $j$ entier.

Nous utiliserons les théorèmes de ce paragraphe essentiellement de deux manières différentes. Ils nous serviront d'une part dans $\S 4.2$ pour démontrer que les modules $\mathcal{D}_{\infty, *}(\mathcal{D})$ sont de rang optimal et pour construire des éléments de $\mathcal{D}_{\infty, g}^{1}(\mathcal{D})$ qui ne sont pas dans $\mathcal{D}_{\infty, f}(\mathcal{D})$ (proposition 4.1.1). Ainsi, la construction de l'exponentielle faite dans le $\S 5$ permet d'obtenir « beaucoup de systèmes compatibles de points dans la cohomologie galoisienne ». Le cas plus technique où $\varphi$ admet une valeur propre qui est une puissance de $p$ est donc important car il permet d'exhiber des éléments « $g$ » qui ne sont pas « $f »$, le cas le plus simple étant celui du $(\varphi, N)$-module trivial de dimension 1 et de la fonction de Kubota-Leopoldt.

D'autre part, ils nous permettront dans $\S 5.1$ d'associer naturellement à un élément

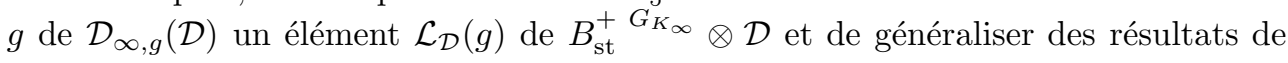
Coleman et Colmez [6].

\subsection{Construction d'éléments de $\mathcal{B}[\log x]$}

Dans la suite $\rho$ est un réel vérifiant $p^{-1 /(p-1)}<\rho<1$.

3.1.1. Lemme. - Si $k$ est un entier positif, la suite $p^{n k} \psi^{n}\left(\log ^{k} x\right)$ converge dans $\mathcal{B}[\log x]$ vers un élément de $\mathcal{B}_{-}[\log x]$ que l'on note $\Theta_{k}$. On a

$$
\psi\left(\Theta_{k}\right)=p^{-k} \Theta_{k}, \quad N \Theta_{k}=k \Theta_{k-1}
$$


et $\Theta_{k}-\log ^{k} x$ est un polynôme en $\log x$ de degré $\leqslant k-2$. Enfin, les $\Theta_{k}$ peuvent être caractérisés comme l'unique suite d'éléments de $\mathcal{B}_{-}[\log x]$ tels que $\psi\left(\Theta_{k}\right)=p^{-k} \Theta_{k}$, $N \Theta_{k}=k \Theta_{k-1}, \Theta_{1}=\log x$.

Ainsi, si $\Theta=\sum_{k=0}^{\infty}\left((-1)^{k} / k !\right) \Theta_{k} \cdot(1 \otimes N)^{k}$ vu comme opérateur de $\mathcal{B}[\log x] \otimes \mathcal{D}$ avec $\mathcal{D}$ un $(\varphi, N)$-module, la suite

$$
\psi^{n}\left(\exp \left(-p^{n} \log x \cdot(1 \otimes N)\right)\right)
$$

converge vers $\Theta$ et $N \Theta=0, \psi(\Theta) \cdot(1 \otimes \varphi)=(1 \otimes \varphi) \Theta$. Nous posons

$$
\Theta_{k}^{(p)}=\left(1-p^{-k} \varphi\right) \Theta_{k}
$$

C'est un élément de $\mathcal{B}_{-}[\log x]^{\psi=0}$ qui est de degré $\leqslant k-1$ en $\log x$. On a

$$
\Theta_{1}^{(p)}=a_{\varphi}=\mathfrak{L}_{1}^{(p)}
$$

Démonstration. - Montrons que $p^{n k} \psi^{n}\left(\log ^{k} x\right)$ a une limite $\Theta_{k}$ dans $\mathcal{B}[\log x]$ lorsque $n \rightarrow \infty$. On a

$$
\begin{aligned}
\epsilon_{n} & \stackrel{\text { déf }}{=} p^{(n+1) k} \psi^{n+1}\left(\log ^{k} x\right)-p^{n k} \psi^{n}\left(\log ^{k} x\right) \\
& =p^{n k} \psi^{n}\left(p^{k} \psi\left(\log ^{k} x\right)-\log ^{k} x\right) .
\end{aligned}
$$

Or

$$
p^{k} \psi\left(\log ^{k} x\right)-\log ^{k} x=\sum_{j=0}^{k-1} p^{k-j}\left(\begin{array}{c}
k \\
j
\end{array}\right) \psi\left(a_{\varphi}^{k-j}\right) \log ^{j} x
$$

est de degré $\leqslant k-1$ en $\log x$ (et même de degré $\leqslant k-2$ car $\psi\left(a_{\varphi}\right)=0$ ) et appartient à $\mathcal{H}_{0}^{[\rho, \infty)}[\log x]$ avec $\rho>p^{-1 /(p-1)}$. On en déduit que $\epsilon_{n}$ appartient à $p^{n k} p^{-n(k-1)} \mathcal{H}_{0}^{[\rho, \infty)}[\log x]$ (lemme 1.2.5), que la suite $\epsilon_{n}$ tend vers 0 et que la suite $p^{n k} \psi^{n}\left(\log ^{k} x\right)$ a une limite qui appartient à $\mathcal{B}_{-}[\log x]$. De plus le coefficient de $\log ^{k-1} x$ étant toujours nul, $\Theta_{k}-\log ^{k} x$ est de degré $\leqslant k-2$ en $\log x$. Calculons $N \Theta$ avec $\Theta=\sum_{k=0}^{\infty}\left((-1)^{k} / k !\right) \Theta_{k}(1 \otimes N)^{k}$ et avec $N=N \otimes 1+1 \otimes N$. On a

$$
\psi \circ N \otimes 1=p N \otimes 1 \circ \psi \text {. }
$$

Donc,

$$
\begin{aligned}
N \psi^{k}\left(\exp \left(-p^{k} \log x(1 \otimes N)\right)\right)= & p^{-k} \psi^{k}\left((N \otimes 1) \exp \left(-p^{k} \log x(1 \otimes N)\right)\right) \\
& +\psi^{k}(1 \otimes N) \exp \left(-p^{k} \log x(1 \otimes N)\right) \\
= & p^{-k} \psi^{k}\left(-p^{k}(1 \otimes N) \exp \left(-p^{k} \log x(1 \otimes N)\right)\right) \\
& \quad+\psi^{k}\left(\exp \left(-p^{k} \log x(1 \otimes N)\right)(1 \otimes N)\right) \\
= & 0 .
\end{aligned}
$$


On en déduit que $N \Theta=0$. Enfin, on a

$$
\begin{aligned}
(1 \otimes \varphi) \Theta & =\lim (1 \otimes \varphi) \psi^{n}\left(\exp \left(-p^{n} \log x(1 \otimes N)\right)\right) \\
& =\lim \psi^{n}\left(\exp \left(-p^{n-1} \log x(1 \otimes N)\right)\right)(1 \otimes \varphi) \\
& =\psi(\Theta)(1 \otimes \varphi) .
\end{aligned}
$$

Pour $k=1, \Theta_{1}=\log x \operatorname{car} \psi(\log x)=p^{-1} \log x$. Montrons par récurrence l'unicité des $\Theta_{k}$ vérifiant les propriétés énoncées. Il s'agit en fait de montrer que si $f$ est un élément de $\mathcal{B}_{-}[\log x]$ tel que $\psi(f)=p^{-k} f$ et $N f=0$ avec $k>0, f$ est nulle. La nullité de $N f$ signifie que $f \in \mathcal{B}_{\text {- }}$ et on utilise alors le lemme 2.4.2.

On déduit de

$$
\log ^{2} x=p^{-2} \varphi \log ^{2} x-2 p^{-1} a_{\varphi} \varphi \log x+a_{\varphi}^{2}
$$

que

$$
\begin{aligned}
\psi\left(\log ^{2} x-p^{-2} \varphi \log ^{2} x\right) & =\psi\left(2 p^{-1} a_{\varphi} \varphi \log x-a_{\varphi}^{2}\right) \\
& =2 p^{-1} \psi\left(a_{\varphi}\right) \log x-\psi\left(a_{\varphi}^{2}\right) \\
& =-\psi\left(a_{\varphi}^{2}\right)
\end{aligned}
$$

Ainsi, en rappelant que $a_{\varphi}$ a été noté aussi $\mathfrak{L}_{1}^{(p t)}$

$$
\begin{aligned}
\Theta_{2} & =\log ^{2} x+\sum_{n=0}^{\infty} \psi^{n}\left(\mathfrak{L}_{1}^{(p)^{2}}\right) \\
& =\mathfrak{L}_{1}^{2}+\sum_{n=0}^{\infty} \psi^{n}\left(\mathfrak{L}_{1}^{(p)^{2}}\right) .
\end{aligned}
$$

On a alors

$$
\frac{1}{2} D\left(\Theta_{2}\right)=\mathfrak{L}_{0} \mathfrak{L}_{1}+\sum_{n=0}^{\infty} \psi^{n}\left(\mathfrak{L}_{0}^{(p)} \mathfrak{L}_{1}^{(p)}\right)
$$

\subsection{Construction d'éléments de $\mathcal{D}_{\infty, e}(\mathcal{D})$}

On note $\overline{\mathcal{D}}$ le $\varphi$-module $\mathcal{D}$ avec opérateur de monodromie $N$ nul. On pose

$$
\begin{aligned}
& \Theta=\sum_{k=0}^{\infty} \frac{(-1)^{k}}{k !} \Theta_{k}(1 \otimes N)^{k} \\
& \mathcal{T}=\sum_{k=0}^{\infty} \frac{1}{k !} \Theta_{k}(1 \otimes N)^{k} .
\end{aligned}
$$

Si $G \in(\mathcal{B} \otimes \mathcal{D})^{\psi=1 \otimes \varphi}, \Theta(G)$ est un élément de $(\mathcal{B}[\log x] \otimes \mathcal{D})^{N=0}$ mais n'est plus dans le noyau de $\psi-1 \otimes \varphi$. Pour obtenir un élément de $(\mathcal{B}[\log x] \otimes \mathcal{D})^{N=0, \psi=1 \otimes \varphi}$, il ne reste plus qu'à appliquer $\lim _{k \rightarrow \infty}(1 \otimes \varphi)^{-k} \psi^{k}$ si cela converge. 


\subsubsection{THÉORÈME}

1) Soit $G$ un élément de $(\mathcal{H} \otimes \mathcal{D})^{\psi=1 \otimes \varphi}$ tel que $(1-\Phi) G$ soit d'ordre $\leqslant 0^{-}$. Alors, la suite $(1 \otimes \varphi)^{-k} \psi^{k}(\Theta(G))$ converge dans $\mathcal{B}[\log x]$ vers un élément

$$
\widetilde{\mathcal{N}}_{\mathcal{D}}(G) \in \widetilde{\mathcal{D}}_{\infty}(\mathcal{D})=(\mathcal{B}[\log x] \otimes \mathcal{D})^{N=0, \psi=1 \otimes \varphi}
$$

tel que $(1-\Phi) \widetilde{\mathcal{N}}_{\mathcal{D}}(G)$ soit d'ordre $\leqslant 0^{-}$et tel que

$$
\widetilde{\mathcal{N}}_{\mathcal{D}}(G) \equiv G \bmod \mathcal{B}[\log x] \otimes N \mathcal{D} .
$$

De plus, $\widetilde{\mathcal{N}}_{\mathcal{D}}(G)$ est l'unique élément de $\widetilde{\mathcal{D}}_{\infty}(\mathcal{D})$ tel que $\widetilde{\mathcal{N}}_{\mathcal{D}}(G)-\Theta G$ soit d'ordre $\leqslant(-1)^{-}$. Enfin, $\widetilde{\mathcal{N}}_{\mathcal{D}}$ est une bijection de l'ensemble des éléments $G$ de $(\mathcal{H} \otimes \mathcal{D})^{\psi=1 \otimes \varphi}$ tels que $(1-\Phi) G$ soit d'ordre $\leqslant 0^{-}$sur l'ensemble des éléments $G$ de $\widetilde{\mathcal{D}}_{\infty}(\mathcal{D})$ tels que $(1-\Phi) G$ soit d'ordre $\leqslant 0^{-}$.

2) Soit $G \in(\mathcal{B}[\log x] \otimes \mathcal{D})^{N=0, \psi=1 \otimes \varphi}$ et tel que $(1-\Phi) G$ soit d'ordre $\leqslant 0^{-}$. La suite $(1 \otimes \varphi)^{-n} \psi^{n}(\mathcal{T}(G))$ converge dans $\mathcal{B} \otimes \mathcal{D}$ vers un élément $\widetilde{\mathcal{M}}_{\mathcal{D}}(G)$ de $(\mathcal{H} \otimes \mathcal{D})^{\psi=1 \otimes \varphi}$ tel que $(1-\Phi) \widetilde{\mathcal{M}}_{\mathcal{D}}(G)$ soit d'ordre $\leqslant 0^{-}$. De plus, $\widetilde{\mathcal{M}}_{\mathcal{D}}(G)$ est l'unique élément de $(\mathcal{B} \otimes \mathcal{D})^{\psi=1 \otimes \varphi}$ tel que $G-\Theta \widetilde{\mathcal{M}}_{\mathcal{D}}(G)$ soit d'ordre $\leqslant(-1)^{-}$.

\subsubsection{RemarqueS}

1) Soit $G$ un élément de $\mathcal{B} \otimes \mathcal{D}$. Si $t_{k}$ est une famille d'éléments de $\mathcal{B}[\log x]$ vérifiant $N t_{k}=k t_{k-1}$,

$$
\sum_{j=0}^{\infty} \frac{(-1)^{j}}{j !} t_{j}(1 \otimes N)^{j} G
$$

est un élément de $(\mathcal{B}[\log x] \otimes \mathcal{D})^{N=0}$. Le premier choix possible est $t_{k}=\log ^{k} x$. Mais le comportement relativement à l'opérateur $\psi$ est compliqué. Nous l'avions utilisé dans une première rédaction. L'équation fonctionnelle $\psi\left(\Theta_{k}\right)=p^{-k} \Theta_{k}$ rend les calculs plus aisés si $t_{k}=\Theta_{k}$. Comme $\Theta_{k}-\log ^{k} x$ est de degré $\leqslant k-1$ par rapport à log $x$, les $\Theta_{k}$ forment une base de $\mathcal{B}[\log x]$ sur $\mathcal{B}$.

2) Lorsque $N G=0$, on a $\widetilde{\mathcal{N}}_{\mathcal{D}}(G)=G$. Supposons que $N=0$ sur $\mathcal{D}$. Les éléments de $\mathcal{D}_{\infty}(\mathcal{D})$ d'ordre $\leqslant 0^{-}$sont dans ce cas contenus dans $\mathcal{H}^{\psi=0} \otimes \mathcal{D}$ car

$$
\left\|(1 \otimes \varphi)^{-n} d \otimes D^{i}\left(a_{\varphi}\right)\right\|_{\rho_{n}} \geqslant C\left\|p^{-i n} D^{i}\left(a_{\varphi}\right)\right\|_{1}=p^{i n}\left\|D^{i}\left(a_{\varphi}\right)\right\|_{1} .
$$

Ainsi, les éléments de $(\mathcal{B} \otimes \mathcal{D})^{\psi=1 \otimes \varphi}$ tels que $(1-\Phi) G$ soit d'ordre $\leqslant 0^{-}$appartiennent nécessairement à $\mathcal{H} \otimes \mathcal{D}$ (voir aussi lemme 3.3.6).

3) Si $\left\|\varphi^{-1}\right\|<1$, tout élément de $\mathbb{Z}_{p} \llbracket x \rrbracket \otimes \mathcal{D}$ est d'ordre $\leqslant 0^{-}$.

4) Supposons $\mathcal{D}$ est de dimension $2: \mathcal{D}=K e_{1} \oplus K e_{2}$ avec $\varphi e_{1}=p^{-k-1} e_{1}$, $\varphi e_{2}=p^{-k} e_{2}, N e_{2}=e_{1}, N e_{1}=0$. Calculons les conditions imposées sur $g=$ $g_{1} e_{1}+g_{2} e_{2}$ avec $(1-\Phi) G=g$. On a

$$
(1 \otimes \varphi)^{-n}(g)=p^{(k+1) n} g_{1} e_{1}+p^{k n} g_{2} e_{2} .
$$

La condition est donc que $\left\|p^{(k+1) n} g_{1}\right\|_{\rho^{1 / p^{n}}}$ et $\left\|p^{k n} g_{2}\right\|_{\rho^{1 / p^{n}}}$ tendent vers 0 lorsque $n \rightarrow \infty$. Donc, $g$ est d'ordre $0^{-}$si et seulement si $g_{1}$ est $o\left(\log ^{k+1}(1+x)\right)$ et si $g_{2}$ est 
un $o\left(\log ^{k}(1+x)\right)$. Pour que $g_{2}$ soit non nul, il est nécessaire que $k>0$. Il s'agit de limitations sévères que l'on peut toujours contourner en tordant $\mathcal{D}$.

Démonstration. - Posons $g=(1-\Phi) G$. Montrons que si

$$
U_{k}=(1 \otimes \varphi)^{-k} \psi^{k}(\Theta(G)),
$$

$U_{k+1}-U_{k}$ tend vers 0 lorsque $k \rightarrow \infty$. On a

$$
U_{k+1}-U_{k}=(1 \otimes \varphi)^{-k} \psi^{k}\left((1 \otimes \varphi)^{-1} \psi-1\right) \Theta(G) .
$$

Or

$$
\begin{aligned}
\Theta(G) & =\sum_{j=0}^{\infty} \frac{(-1)^{j}}{j !}\left(\Theta_{j}^{(p)}+p^{-j} \varphi \Theta_{j}\right)(1 \otimes N)^{j} G \\
\psi(\Theta(G)) & =\sum_{j=0}^{\infty} \frac{(-1)^{j}}{j !}\left(\psi\left(\Theta_{j}^{(p)}(1 \otimes N)^{j} G\right)+p^{-j} \Theta_{j}(1 \otimes N)^{j} \psi(G)\right) .
\end{aligned}
$$

En remplaçant $G$ par $g+\Phi(G)$ et en utilisant le fait que $\psi\left(\Theta_{j}^{(p)}\right)=0$, on obtient

$$
\begin{aligned}
\psi(\Theta(G)) & =\sum_{j=0}^{\infty} \frac{(-1)^{j}}{j !}\left(\psi\left(\Theta_{j}^{(p)}(1 \otimes N)^{j} g\right)+p^{-j} \Theta_{j}(1 \otimes N)^{j}(1 \otimes \varphi) G\right) \\
& =\psi\left(\sum_{j=0}^{\infty} \frac{(-1)^{j}}{j !} \Theta_{j}^{(p)}(1 \otimes N)^{j} g\right)+(1 \otimes \varphi) \Theta(G)
\end{aligned}
$$

Donc,

$$
U_{k+1}-U_{k}=(1 \otimes \varphi)^{-k} \psi^{k}\left(\Theta^{(p)}(g)\right)
$$

avec

$$
\Theta^{(p)}(g)=\sum_{j=0}^{\infty} \frac{(-1)^{j}}{j !} \Theta_{j}^{(p)}(1 \otimes N)^{j} g
$$

Montrons que

$$
V_{k}=p^{k j}(1 \otimes \varphi)^{-k} \psi^{k}\left(g \Theta_{j}^{(p)}\right)
$$

tend vers 0 lorsque $k \rightarrow \infty$. Comme $\Theta_{j}^{(p)}$ est un polynôme en $\log x$ de degré $\leqslant j-1$ à coefficients dans $\mathcal{H}_{0}^{[\rho, \infty)}$, on a grâce au lemme 1.2 .5 pour $p^{-1 /(p-1)} \leqslant \rho<1$ et en posant $\rho_{k}=\rho^{1 / p^{k}}$ :

$$
\begin{aligned}
\left\|p^{k j}(1 \otimes \varphi)^{-k} \psi^{k}\left(g \Theta_{j}^{(p)}\right)\right\|_{\rho} & \leqslant p^{j k}\left\|p^{k j}(1 \otimes \varphi)^{-k} g \Theta_{j}^{(p)}\right\|_{\rho_{k}} \\
& \leqslant\left\|(1 \otimes \varphi)^{-k} g\right\|_{\rho_{k}}\left\|\Theta_{j}^{(p)}\right\|_{\rho} .
\end{aligned}
$$

Comme $g$ est d'ordre $\leqslant 0^{-},\left\|(1 \otimes \varphi)^{-k} g\right\|_{\rho_{k}}$ tend vers 0 lorsque $k \rightarrow \infty$. On en déduit que $U_{k+1}-U_{k}$ tend vers 0 dans $\mathcal{B}[\log x] \otimes \mathcal{D}$ et donc que la suite $U_{k}$ a une limite que 
l'on note $\widetilde{\mathcal{N}}_{\mathcal{D}}(G)$. Il est clair que $\psi\left(\widetilde{\mathcal{N}}_{\mathcal{D}}(G)\right)=(1 \otimes \varphi) \widetilde{\mathcal{N}}_{\mathcal{D}}(G)$. Comme $\Theta_{0}^{(p)}=0$ et que $U_{k+1}-U_{k}$ appartient à $\mathcal{B}[\log x] \otimes N \mathcal{D}$ pour $k \geqslant 0$,

$$
\begin{aligned}
\widetilde{\mathcal{N}}_{\mathcal{D}}(G) & \equiv U_{0} \bmod \mathcal{B}[\log x] \otimes N \mathcal{D} \\
& \equiv G \bmod \mathcal{B}[\log x] \otimes N \mathcal{D} .
\end{aligned}
$$

Calculons $N U_{k}$ :

$$
N U_{k}=p^{-k}(1 \otimes \varphi)^{-k} \psi^{k}(N(\Theta(G))=0 .
$$

Donc $N \widetilde{\mathcal{N}}_{\mathcal{D}}(G)=0$. Montrons que $(1-\Phi) \widetilde{\mathcal{N}}_{\mathcal{D}}(G)$ est d'ordre $\leqslant 0^{-}$. Montrons d'abord

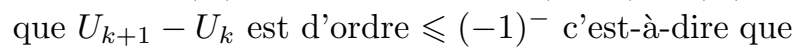

$$
\sup _{k}\left\|p^{n j} p^{-n}(1 \otimes \varphi)^{-n} V_{k}\right\|^{(n)}
$$

tend vers 0 lorsque $n \rightarrow \infty$. Or

$$
\begin{aligned}
\left\|p^{n j} p^{-n}(1 \otimes \varphi)^{-n} V_{k}\right\|^{(n)} & \leqslant\left\|p^{-n+(n+k) j}(1 \otimes \varphi)^{-k-n} \psi^{k}\left(g \Theta_{j}^{(p)}\right)\right\|^{(n)} \\
& \leqslant p^{k j}\left\|p^{-n+(n+k) j}(1 \otimes \varphi)^{-k-n} g \Theta_{j}^{(p)}\right\|^{(n+k)} \\
& \leqslant C_{j} p^{k j}\left\|p^{-n+(n+k) j}(1 \otimes \varphi)^{-k-n} g\right\|^{(n+k)}
\end{aligned}
$$

$\operatorname{avec} C_{j}=\left\|\Theta_{j}^{(p)}\right\|_{\rho}$

$$
\begin{aligned}
& \leqslant C_{j}\left\|p^{n(j-1)}(1 \otimes \varphi)^{-k-n} g\right\|^{(n+k)} \\
& =C_{j}\left\|p^{n(j-1)}(1 \otimes \varphi)^{-k-n} g\right\|_{\rho_{n+k}} .
\end{aligned}
$$

Pour $j \geqslant 1$, ce qu'on peut supposer car $\Theta_{0}=0, n(j-1) \geqslant 0$. Comme $g$ est d'ordre $\leqslant 0^{-}, \sup _{k}\left\|p^{n j} p^{-n}(1 \otimes \varphi)^{-n} V_{k}\right\|^{(n)}$ tend vers 0 lorsque $n \rightarrow \infty$.

Ainsi, $\widetilde{\mathcal{N}}_{\mathcal{D}}(G)-\Theta(G)$ est d'ordre $\leqslant(-1)^{-}$. Pour montrer que $(1-\Phi) \widetilde{\mathcal{N}}_{\mathcal{D}}(G)$ est d'ordre $\leqslant 0^{-}$, il suffit donc de montrer qu'il en est de même de $(1-\Phi) \Theta(G)$. Or

$$
\begin{aligned}
(1-\Phi)(\Theta(G)) & =\Theta(1-\Phi) G+(1-\varphi)(\Theta) \Phi(G) \\
& =\Theta(g)+(1-\varphi)(\Theta) \Phi(G) .
\end{aligned}
$$

Posons $\Theta^{(n)}=\sum_{k}\left((-1)^{k} / k !\right) p^{n k} \Theta_{k}(1 \otimes N)^{k}$. On a

$$
\begin{aligned}
A_{n} & \stackrel{\text { déf }}{=}\left\|(1 \otimes \varphi)^{-n}(1-\varphi)(\Theta)(\Phi(G))\right\|^{(n)} \\
& =\left\|(1-\varphi)\left(\Theta^{(n)}\right)(1 \otimes \varphi)^{-n}(\Phi(G))\right\|^{(n)} \\
& \leqslant C\left\|(1-\varphi) \Theta^{(n)}\right\|^{(n)} ;
\end{aligned}
$$

la dernière inégalité vient de ce que si $h=(1-\Phi) H$ est d'ordre $\leqslant 0^{-}, H$ est d'ordre $\leqslant 0$ : en effet, on tire de $H=\Phi(H)+h$ que

$$
\left\|(1 \otimes \varphi)^{-n} H\right\|_{\rho_{n}} \leqslant \sup \left(\left\|(1 \otimes \varphi)^{-n+1} H\right\|_{\rho_{n-1}},\left\|(1 \otimes \varphi)^{-n} h\right\|_{\rho_{n}}\right) ;
$$

comme $\left\|(1 \otimes \varphi)^{-n} h\right\|_{\rho_{n}}$ tend vers 0 , la suite $\left\|(1 \otimes \varphi)^{-n} H\right\|_{\rho_{n}}$ est bornée. Comme $\Theta_{k}$ appartient à $\mathcal{H}_{0}^{[\rho, \infty)}[\log x],\left\|p^{n k} \Theta_{k}\right\|_{\rho} \leqslant p^{-n k}$, et la limite de $\Theta^{(n)}$ lorsque $n \rightarrow \infty$ 
est $\Theta_{0}=1$. Donc $(1-\varphi) \Theta^{(n)}$ tend vers 0 lorsque $n \rightarrow \infty$. On en déduit que $\lim _{n \rightarrow \infty} A_{n}=0$. D'autre part,

$$
B_{n} \stackrel{\text { déf }}{=}\left\|(1 \otimes \varphi)^{-n} \Theta(g)\right\|^{(n)}=\left\|\Theta^{(n)}\left((1 \otimes \varphi)^{-n} g\right)\right\|^{(n)} .
$$

Comme $\left\|p^{k n} \Theta_{k}^{(n)}\right\|_{\rho} \leqslant p^{-n k}, B_{n}$ tend vers 0 lorsque $n \rightarrow \infty$. Cela démontre que $(1-\Phi) \widetilde{\mathcal{N}}_{\mathcal{D}}(G)$ est d'ordre $\leqslant 0^{-}$.

Finalement, montrons la propriété d'unicité de $\widetilde{\mathcal{N}}_{\mathcal{D}}(G)$. Il s'agit de montrer qu'il n'existe pas de $H \in \mathcal{B}[\log x] \otimes \mathcal{D}$ tel que $\psi(H)=(1 \otimes \varphi) H$ et qui soit d'ordre $\leqslant(-1)^{-}$. On écrit $H$ dans la base des $p^{-k} \varphi \Theta_{k}$ :

$$
H=\sum_{k=0}^{r} H_{k} p^{-k} \varphi \Theta_{k}
$$

avec $H_{r} \neq 0$. L'équation $\psi(H)=(1 \otimes \varphi) H$ implique que

$$
\begin{aligned}
\sum_{k} p^{-k} \psi\left(H_{k}\right) \Theta_{k} & =\sum_{k} p^{-k}(1 \otimes \varphi) H_{k} \varphi \Theta_{k} \\
& \left.=\sum_{k}(1 \otimes \varphi)\right) H_{k}\left(\Theta_{k}-\Theta_{k}^{(p)}\right) .
\end{aligned}
$$

Comme $\Theta_{k}^{(p)}$ est un polynôme de degré $\leqslant k-1$ en $\log x$, on en déduit que

$$
\psi\left(H_{r}\right)=p^{r}(1 \otimes \varphi) H_{r} .
$$

D'où,

$$
\begin{aligned}
\left\|H_{r}\right\|_{\rho} & \leqslant\left\|p^{-r n}(1 \otimes \varphi)^{-n} \psi^{n}\left(H_{r}\right)\right\|_{\rho} \leqslant p^{n}\left\|p^{-r n}(1 \otimes \varphi)^{-n} H_{r}\right\|_{\rho_{n}} \\
& \leqslant\left\|p^{-(r+1) n}(1 \otimes \varphi)^{-n} H_{r}\right\|_{\rho_{n}}
\end{aligned}
$$

ce qui tend vers 0 puisque $H_{r}$ est d'ordre $\leqslant(-1-r)^{-}$par hypothèse. Donc $H_{r}$ est nul ainsi que $H$.

\subsection{Construction d'éléments de $\mathcal{D}_{\infty, g}(\mathcal{D})$}

3.3.1. Posons $\mathcal{U}_{0}(\mathcal{D})=\oplus_{j \geqslant 0} \mathcal{D} \log ^{j}(1+x)=D^{0} \mathcal{U}(\mathcal{D})$ (voir 2.3.2). Rappelons que

$$
\Theta=\sum_{j} \frac{(-1)^{j}}{j !} \Theta_{j}(1 \otimes N)^{j} .
$$

Posons

$$
\int \Theta(H)=\sum_{i \geqslant 1} \frac{(-1)^{i-1}}{i !} \Theta_{i}(1 \otimes N)^{i-1} H .
$$

3.3.2. ThÉORÈme. - Soit $G$ un élément de $\mathcal{B} \otimes \mathcal{D}$ tel que

$$
(\psi-1 \otimes \varphi) G=\psi(\mathfrak{M})
$$


avec $\mathfrak{M} \in \mathcal{U}_{0}(\mathcal{D})$ et soit $\Lambda$ un élément de $\mathcal{U}_{0}(\mathcal{D})$ tel que $(1-p \Phi) \Lambda=N \mathfrak{M}$. On suppose que $g=(1-\Phi) G-\mathfrak{M}$ est d'ordre $\leqslant 0^{-}$. Alors, la suite

$$
(1 \otimes \varphi)^{-k} \psi^{k}(\Theta(G))-\sum_{r=1}^{k}(1 \otimes \varphi)^{-r} \psi^{t}(\mathfrak{M})+(1 \otimes \varphi)^{-k} \psi^{k}\left(\int \Theta(\Lambda)\right)
$$

converge dans $\mathcal{B}[\log x] \otimes \mathcal{D}$ vers un élément $\widetilde{\mathcal{N}}_{\mathcal{D}}(G, \Lambda) \in \mathcal{B}[\log x] \otimes \mathcal{D}$ tel que

$$
\begin{aligned}
& (\psi-1 \otimes \varphi) \widetilde{\mathcal{N}}_{\mathcal{D}}(G, \Lambda)=\psi(\mathfrak{M}) \\
& N \widetilde{\mathcal{N}}_{\mathcal{D}}(G, \Lambda)=\Lambda \\
& \widetilde{\mathcal{N}}_{\mathcal{D}}(G, \Lambda) \equiv G+\log x \cdot \Lambda \bmod \mathcal{B}[\log x] \otimes N \mathcal{D}
\end{aligned}
$$

et tel que $(1-\Phi) \widetilde{\mathcal{N}}_{\mathcal{D}}(G, \Lambda)-\mathfrak{M}$ soit d'ordre $\leqslant 0^{-}$.

Donnons la valeur de $\widetilde{\mathcal{N}}_{\mathcal{D}}$ dans quelques cas particuliers.

1) Lorsque $N=0$ sur $\mathcal{D}$, on a $\widetilde{\mathcal{N}}_{\mathcal{D}}(G, \Lambda)=G+\Lambda \cdot \log x$. En effet, dans ce cas $\Theta(G)=G$.

2) Prenons $G=0$; dans ce cas $\Lambda$ vérifie $\Phi(\Lambda)=p^{-1} \Lambda$. On a alors $\widetilde{\mathcal{N}}_{\mathcal{D}}(0, \Lambda)=\int \Theta(\Lambda)$.

3) Prenons $G=U \in \mathcal{U}_{0}(\mathcal{D}) ; \mathfrak{M}$ vaut alors $(1-\Phi) U$. L'élément $\Lambda$ doit vérifier $(1-p \Phi) \Lambda=N \mathfrak{M}=(1-p \Phi) N U$. On a donc $\Lambda=N U+\Lambda_{0}$ avec $\Phi\left(\Lambda_{0}\right)=p^{-1} \Lambda_{0}$. Alors,

$$
\begin{aligned}
\tilde{\mathcal{N}}_{\mathcal{D}}(U, \Lambda) & =\widetilde{\mathcal{N}}_{\mathcal{D}}(U, N U)+\widetilde{\mathcal{N}}_{\mathcal{D}}\left(0, \Lambda_{0}\right) \\
& =U+\int \Theta\left(\Lambda_{0}\right)=\Theta(U)+\int \Theta(\Lambda) .
\end{aligned}
$$

Ces formules se démontrent facilement à l'aide du formulaire du lemme 3.3.7.

3.3.3. ThÉORÈme (SUITE). - $\widetilde{\mathcal{N}}_{\mathcal{D}}$ est un isomorphisme de l'espace vectoriel des couples $(G, \Lambda)$ avec $G \in \mathcal{B} \otimes \mathcal{D}, \Lambda \in \mathcal{U}_{0}(\mathcal{D})$ vérifiant les conditions

$$
\left\{\begin{array}{l}
(\psi-1 \otimes \varphi) G=\psi(\mathfrak{M}) \text { avec } \mathfrak{M} \in \mathcal{U}_{0}(\mathcal{D}), \\
(1-p \Phi) \Lambda=N \mathfrak{M}, \\
(1-\Phi) G-\mathfrak{M} \text { est d'ordre } \leqslant 0^{-}
\end{array}\right.
$$

sur l'espace vectoriel des $G \in \mathcal{B}[\log x] \otimes \mathcal{D}$ vérifiant

$$
\left\{\begin{array}{l}
(\psi-1 \otimes \varphi) G=\psi(\mathfrak{M}) \text { avec } \mathfrak{M} \in \mathcal{U}_{0}(\mathcal{D}), \\
N G \in \mathcal{U}_{0}(\mathcal{D}), \\
(1-\Phi) G-\mathfrak{M} \text { est d'ordre } \leqslant 0^{-}
\end{array}\right.
$$

Nous noterons $\mathfrak{M}(G)$ (resp. $\Lambda(G)$ ) l'élément de $\mathcal{U}_{0}(\mathcal{D})$ caractérisé par

$$
\psi(\mathfrak{M}(G))=(\psi-1 \otimes \varphi) G \quad(\text { resp. } N G=\Lambda(G)) .
$$


Pour démontrer la bijectivité, nous construirons une application analogue dans l'autre sens, qui, bien que n'étant pas l'inverse de $\widetilde{\mathcal{N}}_{\mathcal{D}}$ en général, permettra de montrer la bijectivité de celle-ci.

Soit $\mathcal{E}_{0}(\mathcal{D})$ l'espace vectoriel des $G \in \mathcal{B} \otimes \mathcal{D}$ tels que $(\psi-1 \otimes \varphi) G=\psi(\mathfrak{M})$ avec $\mathfrak{M} \in \mathcal{U}_{0}(\mathcal{D})$. Considérons le complexe $\mathfrak{C}_{\geqslant 0}(\mathcal{B} \otimes \mathcal{D})$

$$
\begin{array}{ccccc}
\mathcal{U}_{0}(\mathcal{D}) & \longrightarrow & \mathcal{E}_{0}(\mathcal{D}) \times \mathcal{U}_{0}(\mathcal{D}) & \longrightarrow & \mathcal{U}_{0}(\mathcal{D}) \\
U & \longmapsto & (U, N U) & & \\
(G, \Lambda) & & \longrightarrow & N \mathfrak{M}(G)-(1-p \Phi) \Lambda
\end{array}
$$

et $\mathcal{C}_{\geqslant 0}(\mathcal{B} \otimes \mathcal{D})$ l'image de son premier groupe de cohomologie par l'application $(G, \Lambda) \mapsto(1-\Phi) G-\mathfrak{M}(G)$. On vérifie facilement que l'on a on a une suite exacte naturelle

$$
0 \longrightarrow \mathcal{D}_{\infty, f}(\overline{\mathcal{D}}) \longrightarrow \mathcal{C}_{\geqslant 0}(\mathcal{B} \otimes \mathcal{D}) \longrightarrow\left(\mathcal{U}_{0}(\mathcal{D}) / N \mathcal{U}_{0}(\mathcal{D})\right)^{\Phi=p^{-1}} \longrightarrow 0
$$

Notons $\mathcal{C}_{\geqslant 0}(\mathcal{B} \otimes \mathcal{D})^{(0)}$ (resp. $\left.\mathcal{D}_{\infty, g}(\mathcal{D})^{(0)}\right)$ le sous- $\mathbb{Q}_{p}$-espace vectoriel de $\mathcal{C}_{\geqslant 0}(\mathcal{B} \otimes \mathcal{D})$ (resp. de $\left.\mathcal{D}_{\infty, g}(\mathcal{D})\right)$ des classes des $(G, \Lambda)$ tels que $(1-\Phi) G-\mathfrak{M}(G)$ soit d'ordre $\leqslant 0^{-}$ (resp. des éléments $g$ d'ordre $\leqslant 0^{-}$). Alors, le composé de $\widetilde{\mathcal{N}}_{\mathcal{D}}$ avec

$$
G \longmapsto(1-\Phi) G-\mathfrak{M}(G)
$$

induit une application linéaire que l'on note $\mathcal{N}_{\mathcal{D}}$ de $\mathcal{C}_{\geqslant 0}(\mathcal{B} \otimes \mathcal{D})^{(0)}$ dans $\mathcal{D}_{\infty, g}(\mathcal{D})^{(0)}$ :

$$
\mathcal{N}_{\mathcal{D}}(G, \Lambda)=(1-\Phi) \widetilde{\mathcal{N}}_{\mathcal{D}}(G, \Lambda)-\mathfrak{M}(G)
$$

Si $\mathcal{D}_{\infty, g}(\mathcal{D})_{\geqslant 0}^{(0)}$ désigne le sous-module de $\mathcal{D}_{\infty, g}(\mathcal{D})^{(0)}$ formé des éléments $g$ tels que $\mathcal{R}_{\mathcal{D}}(g) \in \mathcal{C}_{\infty, \geqslant 0}(\mathcal{D})$, on obtient la suite suivante du théorème :

3.3.4. ThÉORÈme (SUITE). - $\mathcal{N}_{\mathcal{D}}$ est une bijection de $\mathcal{C}_{\geqslant 0}(\mathcal{B} \otimes \mathcal{D})$ sur $\mathcal{D}_{\infty, g}(\mathcal{D})_{\geqslant 0}^{(0)}$ et on a

$$
\begin{aligned}
\mathcal{N}_{\mathcal{D}}(G, \Lambda) & \equiv(1-\Phi) G-\mathfrak{M}(G)+\mathfrak{L}_{1}^{(p)} \cdot \Lambda \bmod \mathcal{B}[\log x] \otimes N \mathcal{D} \\
\mathcal{R}_{\mathcal{D}}(g) & \equiv(\Lambda, \mathfrak{M}(G)) \in \mathcal{C}_{\infty}(\mathcal{D})
\end{aligned}
$$

Remarquons que si l'on remplace $\mathcal{D}$ par $\mathcal{D}[-u]$ pour $u \gg 0$, on peut supposer que $\mathcal{D}_{\infty, g}(\mathcal{D})_{\geqslant 0}^{(0)}=\mathcal{D}_{\infty, g}(\mathcal{D})^{(0)}$. De même si $g$ est un élément d'ordre $\leqslant v^{-}$, il devient d'ordre $\leqslant 0^{-}$dans $\mathcal{D}_{\infty, g}(\mathcal{D}[-v])$.

Cette bijection n'est cependant pas parfaite car elle n'est pas compatible avec l'action de $G_{\infty}$.

3.3.5. Lorsque $N=0$ sur $\mathcal{D}$, on obtient simplement une bijection de $\mathcal{D}_{\infty, f}(\mathcal{D})^{(0)} \times$ $\mathcal{U}_{0}(\mathcal{D})^{\Phi=p^{-1}}$ sur $\mathcal{D}_{\infty, g}(\mathcal{D})^{(0)}$ donnée par

$$
\mathcal{N}_{\mathcal{D}}(g, \Lambda)=g+\mathfrak{L}_{1}^{(p)} \cdot \Lambda
$$

C'est exactement le scindage de la suite exacte (2.4.2) (voir aussi [24])

$$
0 \longrightarrow \mathcal{D}_{\infty, f}(\mathcal{D}) \longrightarrow \mathcal{D}_{\infty, g}(\mathcal{D}) \longrightarrow \mathcal{U}_{0}(\mathcal{D})^{\Phi=p^{-1}} \longrightarrow 0
$$


3.3.6. Enfin, remarquons que grâce au lemme suivant, si $(G, \Lambda)$ vérifie les conditions $(\mathrm{H}), G$ appartient en fait à $\mathcal{H} \otimes \mathcal{D}$.

Lemme. - Soit $H \in \mathcal{B} \otimes \mathcal{D}$ tel que $(1-\Phi) H-\mathfrak{M}$ soit d'ordre $\leqslant 0^{-}$avec $\mathfrak{M} \in \mathcal{U}(\mathcal{D})$. Alors $H \in \mathcal{H} \otimes \mathcal{D}$.

Démonstration. - Pour démontrer le lemme, on peut utiliser le calcul fait dans le cas où $N=0$. On peut aussi le faire directement. Posons $h=(1-\Phi) H$. C'est un élément d'ordre $\leqslant 0^{-}$. Écrivons $h=h_{+}+h_{-}$avec $h_{+} \in \mathcal{B}_{+}$et $h_{-} \in \mathcal{B}_{-}$d'ordre $\leqslant 0^{-}$ et $H=H_{+}+H_{-}$. On a alors $(1-\Phi) h_{-}=0$. Montrons que si $\mathcal{D}^{\prime}$ est le $\varphi$-support de $h_{-},\left|\varphi^{-1}\right|_{\mathcal{D}^{\prime}}<1$. En effet, si $h_{-}=\sum_{k=1}^{\infty} a_{k} / x^{k}$,

$$
\left\|(1 \otimes \varphi)^{-n} h_{-}\right\|_{\rho_{n}} \geqslant\left\|(1 \otimes \varphi)^{-n} h_{-}\right\|_{1} \geqslant\left|\varphi^{-n} a_{k}\right| \text {. }
$$

On en déduit que pour tout $k \geqslant 1,\left|\varphi^{-n} a_{k}\right|$ tend vers 0 lorsque $n \rightarrow \infty$ et donc que $\left|\varphi^{-n} d\right|$ tend vers 0 pour tout $d$ appartenant à $\mathcal{D}^{\prime}$. Ainsi, $\left|\varphi^{-1}\right|_{\mathcal{D}^{\prime}}<1$. Comme $\operatorname{ker}\left(1-\Phi \mid \mathcal{B}_{-} \otimes \mathcal{D}\right)$ est nul, le $\varphi$-support de $H_{-}$est $\mathcal{D}^{\prime}$. D'autre part, comme $\psi$ conserve $\mathcal{B}_{ \pm}, \psi\left(H_{-}\right)=(1 \otimes \varphi) H_{-}$et

$$
\begin{aligned}
\left\|H_{-}\right\|_{\rho} & =\left\|(1 \otimes \varphi)^{-n} \psi^{n}\left(H_{-}\right)\right\|_{\rho} \leqslant\left\|(1 \otimes \varphi)^{-n} H_{-}\right\|_{\rho} \\
& \leqslant\left|\varphi^{-n}\right|_{\mathcal{D}^{\prime}}\left\|H_{-}\right\|_{\rho} .
\end{aligned}
$$

Comme $\left|\varphi^{-n}\right|_{\mathcal{D}^{\prime}}$ tend vers 0 lorsque $n \rightarrow \infty$, on en déduit que $H_{-}$est nul. Donc $H \in \mathcal{H} \otimes \mathcal{D}$.

Passons à la démonstration du théorème 3.3.2 en commençant par un lemmeformulaire :

\subsubsection{LEMME}

1) Si $b \in \mathcal{U}_{0}(\mathcal{D}), \psi \circ(\varphi \otimes 1)(b)=(\varphi \otimes 1) \circ \psi(b)=b$.

2) Si $b \in \mathcal{U}_{0}(\mathcal{D})$ et $f \in \mathcal{B}[\log x]$, on $a \psi(f b)=\psi(f) \psi(b)$. En particulier, $\psi\left(\Theta_{j} b\right)=p^{-j} \Theta_{j} \psi(b)$ et

$$
\begin{aligned}
(1 \otimes \varphi)^{-1} \psi(\Theta(b)) & =\Theta\left((1 \otimes \varphi)^{-1} \psi(b)\right), \\
(1 \otimes \varphi)^{-1} \psi\left(\int \Theta(b)\right) & =p^{-1} \int \Theta\left((1 \otimes \varphi)^{-1} \psi(b)\right) .
\end{aligned}
$$

$$
\begin{aligned}
(1 \otimes N) \Theta(G) & =\Theta((1 \otimes N) G), \\
N \Theta(G) & =\Theta((N \otimes 1) G) .
\end{aligned}
$$

$$
(1 \otimes N) \int \Theta(H)=\int \Theta((1 \otimes N) H)=H-\Theta(H),
$$$$
(N \otimes 1) \int \Theta(H)=\Theta(H)+\int \Theta((N \otimes 1) H),
$$$$
N \int \Theta(H)=H+\int \Theta((N \otimes 1) H) \text {. }
$$

En particulier, si $H \in \mathcal{B} \otimes \mathcal{D}, N \int \Theta(H)=H=(N \otimes 1) \int \Theta(H)$. 
3.3.8. Posons $\Psi=(1 \otimes \varphi)^{-1} \psi$. Posons $U_{k}^{\prime}=\Psi^{k}(\Theta(G))$ et

$$
U_{k}=U_{k}^{\prime}-\sum_{t=1}^{k} \Psi^{t}(\mathfrak{M})+\Psi^{k}\left(\int \Theta \Lambda\right)
$$

et commençons par calculer $U_{k+1}^{\prime}-U_{k}^{\prime}$. On a comme dans le $\S 3.2$

$$
\psi(\Theta(G))=\sum_{j=0}^{\infty} \frac{(-1)^{j}}{j !}\left(\psi\left(\Theta_{j}^{(p)}(1 \otimes N)^{j} G\right)+p^{-j} \Theta_{j}(1 \otimes N)^{j} \psi(G)\right) .
$$

En remplaçant $G$ par $g+\Phi(G)+\mathfrak{M}$ et $\psi(G)$ par $(1 \otimes \varphi) G+\psi(\mathfrak{M})$ et en utilisant le lemme 3.3.7 et le fait que $\psi\left(\Theta_{j}^{(p)}\right)=0$, on obtient

$$
\begin{aligned}
\psi(\Theta(G)) & =\sum_{j=0}^{\infty} \frac{(-1)^{j}}{j !}\left(\psi\left(\Theta_{j}^{(p)}(1 \otimes N)^{j} g\right)+p^{-j} \Theta_{j}(1 \otimes N)^{j}(1 \otimes \varphi) G\right) \\
& +\sum_{j=0}^{\infty} \frac{(-1)^{j}}{j !} p^{-j} \Theta_{j}(1 \otimes N)^{j} \psi(\mathfrak{M}) \\
& =\psi\left(\Theta^{(p)}(g)\right)+(1 \otimes \varphi) \Theta(G)+\psi(\Theta(\mathfrak{M})) .
\end{aligned}
$$

D'où

$$
U_{k+1}^{\prime}-U_{k}^{\prime}=\Psi^{k+1}\left(\Theta^{(p)}(g)\right)+\Psi^{k+1}(\Theta(\mathfrak{M}))
$$

D'autre part,

$$
\begin{aligned}
(\Psi-1) \int \Theta(\Lambda) & =\int \Theta\left(p^{-1} \Psi(\Lambda)-\Lambda\right)=\int \Theta\left(p^{-1} \Psi((1-p \Phi) \Lambda)\right) \\
& =\int \Theta\left(p^{-1} \Psi((1 \otimes N) \mathfrak{M})\right)=\Psi\left(\int \Theta((1 \otimes N) \mathfrak{M})\right) \\
& =\Psi(\mathfrak{M}-\Theta(\mathfrak{M})) .
\end{aligned}
$$

D'où

$$
\Psi^{k+1}\left(\int \Theta \Lambda\right)-\Psi^{k}\left(\int \Theta \Lambda\right)=\Psi^{k+1}(\mathfrak{M}-\Theta(\mathfrak{M}))
$$

On déduit des équations (3.3.4) et (3.3.5) que

$$
\begin{aligned}
U_{k+1}-U_{k} & =\Psi^{k+1}\left(\Theta^{(p)}(g)\right) \\
& =\sum_{j=0}^{\infty} \frac{(-1)^{j}}{j !}(1 \otimes N)^{j} p^{k j} \Psi^{k}\left(g \Theta_{j}^{(p)}\right) .
\end{aligned}
$$

Le fait que $g$ soit d'ordre $0^{-}$implique comme dans $\S 3.2$ que le premier terme tend aussi vers 0 . On en déduit que la suite $U_{k}$ admet une limite dans $\mathcal{B}[\log x] \otimes \mathcal{D}$ que l'on note $\widetilde{\mathcal{N}}_{\mathcal{D}}(G, \Lambda)$. Vérifions qu'elle a les propriétés voulues. On a $N \Theta(G)=0$ car $(N \otimes 1) G=0$. De même,

$$
N \Psi^{k}\left(\int \Theta(\Lambda)\right)=p^{-k} \Psi^{k}\left(N \int \Theta(\Lambda)\right)=p^{-k} \Psi^{k}(\Lambda)
$$


et

$$
\begin{aligned}
N\left(\sum_{t=1}^{k} \Psi^{t}(\mathfrak{M})\right) & =\sum_{t=1}^{k} p^{-t} \Psi^{t}((1-p \Phi) \Lambda) \\
& =p^{-k} \Psi^{k}(\Lambda)-\Lambda
\end{aligned}
$$

D'où, $N U_{k}=\Lambda$ et en passant à la limite $N \widetilde{\mathcal{N}}_{\mathcal{D}}(G, \Lambda)=\Lambda$. D'autre part,

$$
\Psi\left(U_{k}\right)-U_{k+1}=\Psi(\mathfrak{M}) .
$$

Donc en passant à la limite

$$
(\Psi-1)\left(\widetilde{\mathcal{N}}_{\mathcal{D}}(G, \Lambda)\right)=\Psi(\mathfrak{M}) .
$$

et

$$
(\psi-1 \otimes \varphi)\left(\widetilde{\mathcal{N}}_{\mathcal{D}}(G, \Lambda)\right)=\psi(\mathfrak{M}) .
$$

Enfin, on a modulo $\mathcal{B}[\log x] \otimes N \mathcal{D}$

$$
\begin{aligned}
U_{k} & \equiv \Psi^{k}(G)-\sum_{t=1}^{k} \Psi^{t}(\mathfrak{M}) \\
& \equiv G \bmod \mathcal{B}[\log x] \otimes N \mathcal{D}
\end{aligned}
$$

(se déduit de l'équation $\Psi(G)-G=\Psi(\mathfrak{M})$ ). Donc $\widetilde{\mathcal{N}}_{\mathcal{D}}(G, \Lambda) \equiv G \bmod \mathcal{B}[\log x] \otimes N \mathcal{D}$.

Pour l'ordre de $\widehat{g}=(1-\Phi) \widetilde{\mathcal{N}}_{\mathcal{D}}(G, \Lambda)-\mathfrak{M}$, on vérifie comme dans le $\S 3.2$ que $U_{k+1}-U_{k}=\Psi^{k+1}\left(\Theta^{(p)}(g)\right)$ est d'ordre $\leqslant(-1)^{-}$, puis que $(1-\Phi) U_{0}-\mathfrak{M}$ est d'ordre $\leqslant 0^{-}$.

3.3.9. Construction d'une application presque réciproque. - On part maintenant de $G \in \mathcal{B}[\log x] \otimes \mathcal{D}$ tel que $(\psi-1 \otimes \varphi) G=\psi(\mathfrak{M}), N G=\Lambda$ et tel que $(1-\Phi) G-\mathfrak{M}$ soit d'ordre $\leqslant 0^{-}$, c'est-à-dire vérifiant les propriétés $(\mathrm{K})$. On utilise cette fois ci les opérateurs

$$
\mathcal{T}(H)=\sum_{i \geqslant 0} \frac{1}{i !} \Theta_{i}(1 \otimes N)^{i} H
$$

et

$$
\int \mathcal{T}(H)=\sum_{i \geqslant 1} \frac{1}{i !} \Theta_{i}(1 \otimes N)^{i-1} H
$$

Posons $V_{k}^{\prime}=\Psi^{k}(\mathcal{T}(G))$ et

$$
V_{k}=V_{k}^{\prime}-\sum_{t=1}^{k} \Psi^{t}(\mathfrak{M})-\Psi^{k}\left(\int \mathcal{T} \Lambda\right)
$$

et commençons par calculer $V_{k+1}^{\prime}-V_{k}^{\prime}$. On a comme dans le $\S 3.2$

$$
\psi(\mathcal{T}(G))=\sum_{j=0}^{\infty} \frac{1}{j !}\left(\psi\left(\Theta_{j}^{(p)}(1 \otimes N)^{j} G\right)+p^{-j} \Theta_{j}(1 \otimes N)^{j} \psi(G)\right) .
$$


En remplaçant $G$ par $g+\Phi(G)+\mathfrak{M}$ et $\psi(G)$ par $(1 \otimes \varphi) G+\psi(\mathfrak{M})$ et en utilisant le lemme 3.3.7 et le fait que $\psi\left(\mathcal{T}_{j}^{(p)}\right)=0$, on obtient

$$
\begin{aligned}
\psi(\mathcal{T}(G))= & \sum_{j=0}^{\infty} \frac{1}{j !}\left(\psi\left(\Theta_{j}^{(p)}(1 \otimes N)^{j} g\right)+p^{-j} \Theta_{j}(1 \otimes N)^{j}(1 \otimes \varphi) G\right) \\
& +\sum_{j=0}^{\infty} \frac{1}{j !} p^{-j} \Theta_{j}(1 \otimes N)^{j} \psi(\mathfrak{M}) \\
= & \psi\left(\mathcal{T}^{(p)}(g)\right)+(1 \otimes \varphi) \mathcal{T}(G)+\psi(\mathcal{T}(\mathfrak{M})) .
\end{aligned}
$$

D'où

$$
U_{k+1}^{\prime}-U_{k}^{\prime}=\Psi^{k+1}\left(\mathcal{T}^{(p)}(g)\right)+\Psi^{k+1}(\mathcal{T}(\mathfrak{M}))
$$

D'autre part,

$$
(\Psi-1) \int \mathcal{T}(\Lambda)=\psi(\mathcal{T}(\mathfrak{M})-\mathfrak{M})
$$

D'où

$$
\begin{gathered}
\Psi^{k+1}\left(\int \mathcal{T} \Lambda\right)-\Psi^{k}\left(\int \mathcal{T} \Lambda\right) \\
=\Psi^{k+1}(\mathcal{T}(\mathfrak{M})-\mathfrak{M}) .
\end{gathered}
$$

On déduit des équations (3.3.4) et (3.3.6) que

$$
\begin{aligned}
V_{k+1}-V_{k} & =\Psi^{k+1}\left(\mathcal{T}^{(p)}(g)\right) \\
& =\sum_{j=0}^{\infty} \frac{1}{j !}(1 \otimes N)^{j} p^{k j} \Psi^{k}\left(g \Theta_{j}^{(p)}\right) .
\end{aligned}
$$

Le fait que $g$ soit d'ordre $0^{-}$implique comme dans le cas du $\S 3.2$ que le premier terme tend aussi vers 0 . On en déduit que la suite $V_{k}$ admet une limite dans $\mathcal{B}[\log x] \otimes \mathcal{D}$ que l'on note $\widetilde{\mathcal{M}}_{\mathcal{D}}(G)$. Vérifions qu'elle a les propriétés voulues. On a

$$
\begin{aligned}
(N \otimes 1) \mathcal{T}(G) & =\sum_{j=1}^{\infty} \frac{1}{(j-1) !} \Theta_{j-1}(1 \otimes N)^{j} G+\sum_{j=0}^{\infty} \frac{1}{j !} \Theta_{j}(1 \otimes N)^{j}(N \otimes 1) G \\
& =\mathcal{T}(N G)=\mathcal{T}(\Lambda)
\end{aligned}
$$

car $N G=\Lambda$. D'autre part,

$$
\begin{aligned}
(N \otimes 1) \int \mathcal{T}(\Lambda) & =\sum_{j=1}^{\infty} \frac{1}{(j-1) !} \Theta_{j-1}(1 \otimes N)^{j-1} \Lambda \\
& =\mathcal{T}(\Lambda)
\end{aligned}
$$

et

$$
(N \otimes 1) \sum_{t=1}^{k} \Psi^{t}(\mathfrak{M})=0
$$


On en déduit que $(N \otimes 1) V_{k}=0$ et en passant à la limite que

$$
(N \otimes 1) \widetilde{\mathcal{M}}_{\mathcal{D}}(G)=0 \text {. }
$$

Ainsi, $\widetilde{\mathcal{M}}_{\mathcal{D}}(G)$ appartient à $\mathcal{B} \otimes \mathcal{D}$. D'autre part,

$$
\Psi\left(V_{k}\right)-V_{k+1}=\Psi(\mathfrak{M}) .
$$

Donc en passant à la limite

$$
(\psi-1 \otimes \varphi) \widetilde{\mathcal{N}}_{\mathcal{D}}(G, \Lambda)=\psi(\mathfrak{M}) .
$$

Enfin, on a modulo $\mathcal{B} \otimes N \mathcal{D}$

$$
\begin{aligned}
V_{k} & \equiv \Psi^{k}(G)-\sum_{t=1}^{k} \Psi^{t}(\mathfrak{M}) \\
& \equiv \Psi^{k}(G)-\sum_{t=1}^{k} \Psi^{t}(\mathcal{T}(\mathfrak{M})) \\
& \equiv G \bmod \mathcal{B}[\log x] \otimes N \mathcal{D} .
\end{aligned}
$$

Donc $\widetilde{\mathcal{M}}_{\mathcal{D}}(G) \equiv G \bmod \mathcal{B}[\log x] \otimes N \mathcal{D}$. On procède de la même manière que pour $\widetilde{\mathcal{N}}_{\mathcal{D}}$ pour comparer les ordres de $\widehat{g}=(1-\Phi) \widetilde{\mathcal{M}}_{\mathcal{D}}(G)-\mathfrak{M}$ et de $g=(1-\Phi) G-\mathfrak{M}$.

3.3.10. Démonstration de la bijectivité de $\widetilde{\mathcal{N}}_{\mathcal{D}}$. - Lorsque $N=0$ sur $\mathcal{D}, \widetilde{\mathcal{N}}_{\mathcal{D}}$ et $\widetilde{\mathcal{M}}_{\mathcal{D}}$ sont clairement inverses l'une de l'autre.

Supposons que $\widetilde{\mathcal{N}}_{\mathcal{D}}(G, \Lambda)=0$ et soit $\Delta$ le plus petit $(\varphi, N)$-module de $\mathcal{D}$ tel que $G$ et $\Lambda$ appartiennent à $\mathcal{B} \otimes \Delta$. Comme

$$
\widetilde{\mathcal{N}}_{\mathcal{D}}(G, \Lambda)=\widetilde{\mathcal{N}}_{\Delta}(G, \Lambda) \equiv G+\Lambda \cdot \log x \bmod \mathcal{B}[\log x] \otimes N \Delta
$$

et que $G \in \mathcal{B} \otimes \Delta$, on en déduit que $G \in \mathcal{B} \otimes N \Delta$ et que $\Lambda \in \mathcal{U}(N \Delta)$. Comme $N \Delta$ est strictement contenu dans $\Delta$ si $\Delta \neq 0$, on en déduit la nullité de $G$.

Montrons maintenant la surjectivité par récurrence sur le degré de nilpotence de $\mathcal{D}$. Soit $G$ vérifiant les propriétés $(\mathrm{K})$ et $\Lambda=N G$. Soit $G_{0}=\widetilde{\mathcal{M}}_{\mathcal{D}}(G)$. Alors $\left(G_{0}, \Lambda\right)$ vérifie les propriétés $(\mathrm{H})$. On a

$$
\begin{aligned}
\widetilde{\mathcal{N}}_{\mathcal{D}}\left(G_{0}, \Lambda\right) & \equiv G_{0}+\log x \cdot \Lambda \bmod \mathcal{B}[\log x] \otimes N \mathcal{D} \\
& \equiv G+\log x \cdot \Lambda \bmod \mathcal{B}[\log x] \otimes N \mathcal{D} .
\end{aligned}
$$

Alors $G_{1}=G-\widetilde{\mathcal{N}}_{\mathcal{D}}\left(G_{0}, \Lambda\right)$ vérifie les propriétés $(\mathrm{K})$ et appartient à $\mathcal{B}[\log x] \otimes N \mathcal{D}$. En utilisant l'hypothèse de récurrence, on en déduit que $G_{1}$ appartient à l'image de $\widetilde{\mathcal{N}}_{N \mathcal{D}}$ et donc à l'image de $\widetilde{\mathcal{N}}_{\mathcal{D}}$. 


\section{CHAPITRE 4}

\section{THÉORÈMES DE STRUCTURE DES $\mathcal{D}_{\infty, *}(\mathcal{D})$}

\subsection{Lien entre les $\mathcal{D}_{\infty, *}(\mathcal{D})$}

4.1.1. Proposition. - Les suites suivantes de $G_{\infty}$-modules sont exactes :

$$
\begin{gathered}
0 \longrightarrow \mathcal{D}_{\infty, e}(\mathcal{D}) \longrightarrow \mathcal{D}_{\infty, g}(\mathcal{D}) \stackrel{\mathcal{R}_{\mathcal{D}}}{\longrightarrow} \mathcal{C}_{\infty}(\mathcal{D}) \longrightarrow 0 \\
0 \longrightarrow \mathcal{D}_{\infty, f}(\mathcal{D}) \longrightarrow \mathcal{D}_{\infty, g}(\mathcal{D}) \stackrel{\overline{\mathcal{R}}_{\mathcal{D}}}{\longrightarrow}(\mathcal{U}(\mathcal{D}) / N \mathcal{U}(\mathcal{D}))^{\Phi=p^{-1}} \longrightarrow 0 \\
0 \longrightarrow \mathcal{D}_{\infty, e}(\mathcal{D}) \longrightarrow \mathcal{D}_{\infty, f}(\mathcal{D}) \stackrel{\mathcal{R}_{\mathcal{D}}}{\longrightarrow} \mathcal{U}(\mathcal{D})^{N=0} /(1-p \Phi) \mathcal{U}(\mathcal{D})^{N=0} \longrightarrow 0
\end{gathered}
$$

Démonstration. - Il suffit de montrer que $\mathcal{R}_{\mathcal{D}}$ est surjective. On peut tordre $\mathcal{D}$ de manière à ce que $\mathcal{D}^{\varphi=p^{i}}=0$ pour $i \geqslant 0$. On a alors $\mathcal{C}_{\infty}(\mathcal{D})=\mathcal{C}_{\infty, \geqslant 0}(\mathcal{D})$. Soit $(\Lambda, \mathfrak{M}) \in \mathcal{U}(\mathcal{D}) \times \mathcal{U}(\mathcal{D})$ tel que

$$
(1-p \Phi) \Lambda=N \mathfrak{M} .
$$

Il existe $G \in \mathcal{B} \otimes \mathcal{D}$ tel que $(\psi-1 \otimes \varphi) G=\psi(\mathfrak{M})$ (l'existence vient de la surjectivité de $\left.\mathcal{D}_{\infty, f}(\overline{\mathcal{D}}) \longrightarrow \mathcal{U}(\mathcal{D}) /(1-\Phi) \mathcal{U}(\mathcal{D}), \S 2.4\right)$. Quitte à remplacer encore $\mathcal{D}$ par $\mathcal{D}[-u]$ avec $u \geqslant 0$, on peut supposer que l'ordre de $g=(1-\Phi) G-\mathfrak{M}$ est $\leqslant 0^{-}$. Donc $\widetilde{\mathcal{N}}_{\mathcal{D}}(G, \Lambda)$ existe et si $g=(1-\Phi) \widetilde{\mathcal{N}}_{\mathcal{D}}(G, \Lambda)-\mathfrak{M}, \mathcal{R}_{\mathcal{D}}(g)$ est égale à la classe de $(\Lambda, \mathfrak{M})$.

4.1.2. Regardons le cas particulier où $\mathfrak{M}=0$ et $\Phi \Lambda=p^{-1} \Lambda$. Alors,

$$
\mathcal{N}_{\mathcal{D}}(0, \Lambda)=\int \Theta(\Lambda)
$$

$\mathbf{h}(\Lambda)=(1-\Phi) \int \Theta(\Lambda)$ est un élément de $\mathcal{D}_{\infty, g}(\mathcal{D})$ tel que

$$
\mathbf{h}(\Lambda) \equiv \mathfrak{L}_{1}^{(p)} \Lambda \bmod \mathcal{B}[\log x] \otimes N \mathcal{D}
$$

et $\overline{\mathcal{R}}_{\mathcal{D}, 0}(\mathbf{h}(\Lambda))$ est la classe de $\Lambda$ dans $(\mathcal{D} / N \mathcal{D})^{\varphi=p^{-1}}$. On a

$$
\mathbf{h}(\Lambda)=\sum_{k=1}^{\infty} \frac{(-1)^{k-1}}{(k) !} \Theta_{k}^{(p)} N^{k-1} \Lambda .
$$


Par exemple, lorsque $N^{2}=0$, on trouve que

$$
\begin{aligned}
\int \Theta(\Lambda) & =\log x \cdot \Lambda-\frac{\Theta_{2}}{2} \cdot N \Lambda, \\
\mathbf{h}(\Lambda) & =\mathfrak{L}_{1}^{(p)} \cdot \Lambda-\frac{\Theta_{2}^{(p)}}{2} \cdot N \Lambda .
\end{aligned}
$$

\subsection{Rang des $\mathcal{D}_{\infty, *}(\mathcal{D})$}

4.2.1. ThÉORÈme. — Soit $\mathcal{D}$ un $(\varphi, N)$-module de dimension finie. Les $\mathcal{H}\left(G_{\infty}\right)$-modules $\mathcal{D}_{\infty, *}(\mathcal{D})$ pour $* \in\{e, f, g\}$ sont des modules de type fini, sans torsion, de rang égal à $\operatorname{dim}_{\mathbb{Q}_{p}} \mathcal{D}$ et admettant une résolution de longueur finie $(\leqslant 2)$ par des modules libres de type fini.

En particulier, $\operatorname{det} \mathcal{D}_{\infty, *}(\mathcal{D})$ a un sens.

Démonstration. - On note $\Lambda_{\mathbb{Q}_{p}}=\mathbb{Q}_{p} \otimes \mathbb{Z}_{p} \llbracket G_{\infty} \rrbracket$. Nous utiliserons les faits suivants :

(i) Si $f \in \Lambda_{\mathbb{Q}_{p}}, \mathcal{H}\left(G_{\infty}\right) / f \mathcal{H}\left(G_{\infty}\right)=\Lambda_{\mathbb{Q}_{p}} / f \Lambda_{\mathbb{Q}_{p}}$ (par le théorème de préparation de Weierstrass, on se ramène au cas où $f$ est un polynôme (distingué) puis on utilise la division euclidienne d'un élément de $\mathcal{H}\left(G_{\infty}\right)$ par $\left.f,[\mathbf{1}]\right)$.

(ii) Si $M$ est un $\mathcal{H}\left(G_{\infty}\right)$-module de torsion qui est un $\mathbb{Q}_{p}$-espace vectoriel de dimension finie, c'est aussi un $\Lambda_{\mathbb{Q}_{p}}$-module de type fini et il admet donc une résolution de longueur 2 par des modules libres de type fini sur $\mathcal{H}\left(G_{\infty}\right)$. En effet, $M$ est annulé par $P(\gamma-1) \in \mathbb{Q}_{p}[\Gamma]$ où $P$ est le polynôme caractéristique de l'endomorphisme $\gamma-1$ et on utilise le fait que $\mathbb{Z}_{p} \llbracket \Gamma \rrbracket$ est un anneau local régulier de dimension 2 et la remarque précédente.

(iii) Si $M$ est comme précédemment et dans une suite exacte $0 \rightarrow M_{1} \rightarrow M_{2} \rightarrow$ $M \rightarrow 0$, avec $M_{2}$ un $\mathcal{H}\left(G_{\infty}\right)$-module de type fini, alors $M_{1}$ l'est aussi : si $\left(m_{i}\right)$ est un système générateur de $M_{2}$, le fait que $\sum_{i} \alpha_{i} m_{i}$ appartient à $M_{1}$ se traduit par des relations entre les $\alpha_{i}$ engendrées par un nombre fini de relations!

Les quotients $\mathcal{D}_{\infty, g / f}(\mathcal{D})$ et $\mathcal{D}_{\infty, f / e}(\mathcal{D})$ sont des $\Lambda_{\mathbb{Q}_{p}}$-modules de type fini de torsion et en particulier admettent une résolution de longueur 2 par des modules libres de type fini. Cette propriété étant stable par suite exacte et en utilisant (iii), il suffit donc de montrer le théorème pour un des $\mathcal{D}_{\infty, *}(\mathcal{D})$.

Lorsque $N=0$ sur $\mathcal{D}$, le théorème est vrai. Passons au cas général. La suite

$$
0 \longrightarrow \mathcal{D}_{\infty, g}\left(\mathcal{D}^{N=0}\right) \longrightarrow \mathcal{D}_{\infty, g}(\mathcal{D}) \longrightarrow \mathcal{D}_{\infty, f}\left(\mathcal{D} / \mathcal{D}^{N=0}\right) \longrightarrow 0
$$

est exacte à des modules de torsion près qui sont de plus des $\mathbb{Q}_{p}$-espaces vectoriels de dimension finie. Or $\mathcal{D}^{N=0}$ n'est jamais nul (si $\mathcal{D}$ ne l'est pas). On en déduit par récurrence sur la dimension de $\mathcal{D}$ que $\mathcal{D}_{\infty, g}(\mathcal{D})$ est de type fini, de rang inférieur ou égal à $\operatorname{dim}_{\mathbb{Q}_{p}} \mathcal{D}$ et admet une résolution de longueur finie (en fait $\leqslant 2$ ) par des modules libres de type fini. 
Montrons maintenant que $\mathcal{D}_{\infty}(\mathcal{D})$ est un $\mathcal{H}\left(G_{\infty}\right)$-module de rang $\geqslant \operatorname{dim} \mathcal{D}=d$. Quitte à remplacer $\mathcal{D}$ par $\mathcal{D}[-k]$ avec $k \gg 0$, on peut supposer que $\left\|\varphi^{-1}\right\|<1$. Il existe $g_{0} \in \mathbb{Z}_{p} \llbracket x \rrbracket^{\psi=0}$ tel que les $R(d)=g_{0} \otimes d$ avec $d \in \mathcal{D}$ appartiennent à l'image de $1-\Phi$. Par exemple, on prend $\prod_{k \in K}\left(\chi(\gamma)^{k} \gamma-1\right) \cdot(1+x)$ pour $K$ l'ensemble des entiers tels que $\mathcal{D}^{\varphi=p^{-k}}$ est non nul. Notons $\widetilde{R}(d)$ un élément de $(\mathcal{B} \otimes \mathcal{D})^{\psi=1 \otimes \varphi}$ tel que $(1-\Phi) \widetilde{R}(d)=R(d)$.

Comme $N$ est un endomorphisme nilpotent $\operatorname{sur} \mathcal{D}$ et que $N \varphi=p \varphi N$, les sousespaces $\mathcal{D}_{i}=N^{i} \mathcal{D}$ qui sont non nuls forment une suite strictement décroissante de sous-espaces de $\mathcal{D}$ stables par $\varphi$ et par $N$. Choisissons une base $B$ de $\mathcal{D}$ adaptée à la filtration par les $\mathcal{D}_{i}$, c'est-à-dire telle que les $\beta$ de $B$ non nuls modulo $\mathcal{D}_{i}$ forment un système libre dans $\mathcal{D} / \mathcal{D}_{i}$. Les $(R(\beta))_{\beta \in B}$ vérifient alors une propriété analogue : l'ensemble des $R(\beta)$ non nuls modulo $\mathcal{H}^{\psi=0} \otimes \mathcal{D}_{i}$ forme un système libre dans $\mathcal{H}^{\psi=0} \otimes \mathcal{D} / \mathcal{D}_{i}$.

Considérons maintenant les $\widetilde{S}(\beta)=\widetilde{\mathcal{N}}_{\mathcal{D}}(\widetilde{R}(\beta)) \in \widetilde{\mathcal{D}}_{\infty}(\mathcal{D})$ qui existent car les $R(\beta)$ sont d'ordre $\leqslant 0^{-}$, et montrons que les $S(\beta)=(1-\Phi) \widetilde{S}(\beta)$ forment un système libre dans $\mathcal{D}_{\infty}(\mathcal{D})$. Soit $\sum_{\beta \in B^{\prime}} \lambda_{\beta} \cdot S(\beta)=0$ avec $B^{\prime} \subset B$ et $\lambda_{\beta} \neq 0$ pour tout $\beta \in B^{\prime}$. Soit $r$ le plus grand entier tel que les $S(\beta)$ appartiennent tous à $\widetilde{\mathcal{D}}_{\infty}\left(\mathcal{D}_{r}\right)$. On a

$$
\sum_{\beta \in B^{\prime}} \lambda_{\beta} \cdot \widetilde{S}(\beta) \in \operatorname{ker}\left(1-\Phi \mid \widetilde{\mathcal{D}}_{\infty}(\mathcal{D})\right)=0
$$

Passons au quotient par $\mathcal{D}_{r+1}$ et notons $\pi$ la projection. On a

$$
\sum_{\beta \in B^{\prime}} \lambda_{\beta} \cdot \widetilde{\mathcal{N}}_{\mathcal{D}_{r} / \mathcal{D}_{r+1}}(\pi(\widetilde{R}(\beta)))=0
$$

dans $\widetilde{\mathcal{D}}_{\infty}\left(\mathcal{D}_{r} / \mathcal{D}_{r+1}\right)$. Comme $N=0$ sur $\mathcal{D}_{r} / \mathcal{D}_{r+1}, \widetilde{\mathcal{N}}_{\mathcal{D}_{r} / \mathcal{D}_{r+1}}$ est en fait l'identité. On en déduit que dans $\widetilde{\mathcal{D}}_{\infty}\left(\mathcal{D}_{r} / \mathcal{D}_{r+1}\right)$

$$
\sum_{\beta \in B^{\prime}} \lambda_{\beta} \cdot \pi(\widetilde{R}(\beta))=0
$$

En appliquant $1-\Phi$, on a

$$
\sum_{\beta \in B^{\prime}} \lambda_{\beta} \cdot \pi(R(\beta))=0
$$

On en déduit que les $\lambda_{\beta}$ tels que $\beta \in \mathcal{D}_{r}$ sont nuls en contradiction avec l'hypothèse que les $\lambda_{\beta}$ sont tous non nuls. Donc les $(R(\beta))_{\beta \in B}$ forment un système libre de $\mathcal{D}_{\infty, g}(\mathcal{D})$.

On peut retrouver le fait que $\mathcal{D}_{\infty, g}(\mathcal{D})$ admet une résolution libre de longueur $\leqslant 2$ de la manière suivante. Soit $M$ le sous- $\mathcal{H}\left(G_{\infty}\right)$-module libre de $\mathcal{D}_{\infty, g}(\mathcal{D})$ engendré par les $R(\beta)$. On démontre par récurrence comme précédemment qu'il existe $h_{0} \in \mathbb{Z}_{p} \llbracket G_{\infty} \rrbracket$ non diviseur de zéro tel que pour $g \in \mathcal{D}_{\infty, *}(\mathcal{D}), f_{0} \cdot g$ appartient à $M$. Comme les $\mathcal{D}_{\infty, *}(\mathcal{D})$ sont de type fini sur $\mathcal{H}\left(G_{\infty}\right)$, le quotient de $\mathcal{D}_{\infty, *}(\mathcal{D})$ par $\sum \mathcal{H}\left(G_{\infty}\right) R(\beta)$ est en fait un $\Lambda_{\mathbb{Q}_{p}}$-module de type fini et de torsion. Comme $\mathcal{D}_{\infty, g}(\mathcal{D})$ est une extension d'un $\Lambda_{\mathbb{Q}_{p}}$-module de type fini et de torsion par un $\mathcal{H}\left(G_{\infty}\right)$-module libre, il vérifie les conclusions du théorème 4.2.1. 


\subsection{Comportement par suite exacte}

4.3.1. On pose

$$
\begin{aligned}
& \mathcal{D}_{\infty, g}^{1}(\mathcal{D})=\mathcal{D}_{\infty, g}(\mathcal{D}), \\
& \mathcal{D}_{\infty, g}^{2}(\mathcal{D})=\mathcal{U}(\mathcal{D}) /(1-p \Phi, N) \mathcal{U}(\mathcal{D}), \\
& \mathcal{D}_{\infty, g}^{i}(\mathcal{D})=0 \text { pour } i \neq 1,2 .
\end{aligned}
$$

Si $(\alpha): 0 \rightarrow \mathcal{D}_{1} \rightarrow \mathcal{D}_{2} \rightarrow \mathcal{D}_{3} \rightarrow 0$ est une suite exacte de $(\varphi, N)$-modules, on définit une application de connexion

$$
\mathcal{D}_{\infty, g}^{1}\left(\mathcal{D}_{3}\right) \longrightarrow \mathcal{D}_{\infty, g}^{2}(\mathcal{D})
$$

en composant l'application

$$
\mathcal{R}_{\mathcal{D}_{3}}: \mathcal{D}_{\infty, g}^{1}\left(\mathcal{D}_{3}\right) \longrightarrow \mathcal{C}_{\infty}\left(\mathcal{D}_{3}\right)
$$

avec l'application de connexion

$$
\mathcal{C}_{\infty}\left(\mathcal{D}_{3}\right) \longrightarrow \mathcal{U}\left(\mathcal{D}_{1}\right) /(1-p \Phi, N) \mathcal{U}\left(\mathcal{D}_{1}\right)
$$

définie de manière explicite par

$$
(\Lambda, \mathfrak{M}) \longmapsto N \widehat{\mathfrak{M}}-(1-p \Phi) \widehat{\Lambda}
$$

si $\widehat{\Lambda}$ et $\widehat{\mathfrak{M}}$ sont des relèvements de $\Lambda$ et $\mathfrak{M}$ dans $\mathcal{U}\left(\mathcal{D}_{2}\right)$.

4.3.2. Proposition. - Soit $(\alpha): 0 \rightarrow \mathcal{D}_{1} \rightarrow \mathcal{D}_{2} \rightarrow \mathcal{D}_{3} \rightarrow 0$ une suite exacte de $(\varphi, N)$-modules. Alors la suite longue

$$
\ldots \longrightarrow \mathcal{D}_{\infty, g}^{i}\left(\mathcal{D}_{1}\right) \longrightarrow \mathcal{D}_{\infty, g}^{i}\left(\mathcal{D}_{2}\right) \longrightarrow \mathcal{D}_{\infty, g}^{i}\left(\mathcal{D}_{3}\right) \longrightarrow \ldots
$$

est une suite exacte de $\mathcal{H}\left(G_{\infty}\right)$-modules.

Démonstration. - Il est facile de vérifier que la suite est un complexe. Le seul point délicat est l'exactitude de

$$
\mathcal{D}_{\infty, g}^{1}\left(\mathcal{D}_{2}\right) \longrightarrow \mathcal{D}_{\infty, g}^{1}\left(\mathcal{D}_{3}\right) \longrightarrow \mathcal{D}_{\infty, g}^{2}\left(\mathcal{D}_{1}\right) .
$$

Remarquons aussi que l'énoncé est invariant par twist. Commençons par deux cas particuliers. D'abord lorsque $N=0$ sur $\mathcal{D}_{2}$ et donc sur tous les $\mathcal{D}_{i}$, la proposition 4.3.2 a été montrée (proposition 2.4.5). Démontrons maintenant que la suite (4.3.1) (c'est-à-dire en fait la suite (4.3.2)) est exacte lorsque $N=0$ sur $\mathcal{D}_{3}$.

On peut supposer que $\left\|\varphi^{-1}\right\|_{\mathcal{D}_{i}}<1$. Soit $g \in \mathcal{D}_{\infty, g}\left(\mathcal{D}_{3}\right) \subset \mathcal{B}^{\psi=0} \otimes \mathcal{D}_{3}$. Quitte à tordre encore les $\mathcal{D}_{i}$, on peut supposer que $\mathfrak{o}_{\varphi}(g) \leqslant 0^{-}$(pour $u>0$ tel que $\| p^{n u}(1 \otimes$ $\varphi)^{-n} g \|_{\rho_{n}}$ tend vers 0 lorsque $n \rightarrow \infty$, on remplace les $\mathcal{D}_{i}$ par $\left.\mathcal{D}_{i}[-u]\right)$.

Soit $(\Lambda, \mathfrak{M})$ un représentant de $\mathcal{R}_{\mathcal{D}_{3}}(g)$. On peut écrire

$$
g=\Lambda \mathfrak{L}_{1}^{(p)}+g_{1}
$$

avec $g_{1} \in \mathcal{D}_{\infty, f}\left(\mathcal{D}_{3}\right)$ d'ordre $\leqslant 0^{-}$. D'autre part, comme l'image de $g$ dans $\mathcal{U}\left(\mathcal{D}_{1}\right) /(1-\Phi, N) \mathcal{U}\left(\mathcal{D}_{1}\right)$ est nulle, il existe $\widehat{\Lambda}$ et $\widehat{\mathfrak{M}}$ dans $\mathcal{U}\left(\mathcal{D}_{2}\right)$ relevant $\Lambda$ et $\mathfrak{M}$ 
et vérifiant $(1-p \Phi) \widehat{\Lambda}=N \widehat{\mathfrak{M}}$. Il existe un relèvement $\widehat{g}_{1}$ de $g_{1}$ dans $\mathcal{D}_{\infty, f}\left(\overline{\mathcal{D}}_{2}\right)$ : il existe $G_{2} \in \mathcal{H} \otimes \mathcal{D}_{2}$ tel que $(1-\Phi) G_{2}=\widehat{g}_{1}-\widehat{\mathfrak{M}}$. Alors, $\widetilde{\mathcal{N}}_{\mathcal{D}_{2}}\left(G_{2}, \widehat{\Lambda}\right)$ existe et $\widetilde{g}_{1}=(1-\Phi) \widetilde{\mathcal{N}}_{\mathcal{D}_{2}}\left(G_{2}, \widehat{\Lambda}\right)-\widehat{\mathfrak{M}}$ est un élément de $\mathcal{D}_{\infty, g}\left(\mathcal{D}_{2}\right)$ tel que

$$
\widetilde{g}_{1} \equiv \widehat{g}_{1}+\mathfrak{L}_{1}^{(p)} \cdot \widehat{\Lambda} \bmod \mathcal{B}[\log x] \otimes N \mathcal{D}_{2} .
$$

L'image de $\widetilde{g}_{1}$ dans $\mathcal{D}_{\infty, g}\left(\mathcal{D}_{3}\right)$ est donc $g$, ce qui démontre l'exactitude de (4.3.2).

Démontrons le cas général par récurrence sur le degré de nilpotence de $N$ sur $\mathcal{D}_{2}$. Il existe un diagramme à 9 termes commutatif dont les suites et les colonnes sont exactes

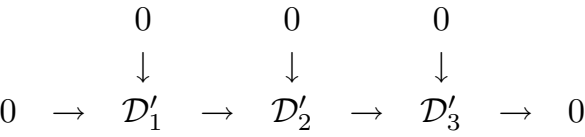

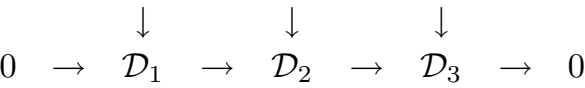

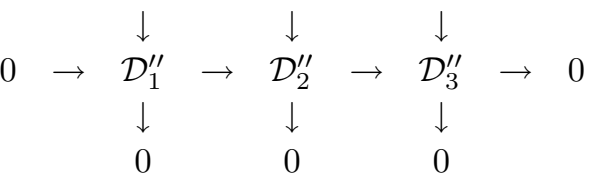

et tel que le degré de nilpotence de $N$ sur $\mathcal{D}_{2}^{\prime \prime}$ et sur $\mathcal{D}_{2}^{\prime}$ est strictement inférieur à celui sur $\mathcal{D}_{2}$. Par exemple, on peut prendre $\mathcal{D}_{i}^{\prime \prime}=\mathcal{D}_{i} / N \mathcal{D}_{i}$ pour $i=2$ et $3, \mathcal{D}_{1}^{\prime \prime}$ le noyau de $\mathcal{D}_{2}^{\prime \prime} \rightarrow \mathcal{D}_{3}^{\prime \prime}$ (c'est aussi $\mathcal{D}_{1} / \mathcal{D}_{1} \cap N \mathcal{D}_{2}$ ), $\mathcal{D}_{i}^{\prime}=N \mathcal{D}_{i}$ pour $i=2$ et $3, \mathcal{D}_{1}^{\prime}=\mathcal{D}_{1} \cap N \mathcal{D}_{2}$. On en déduit un diagramme commutatif tordu

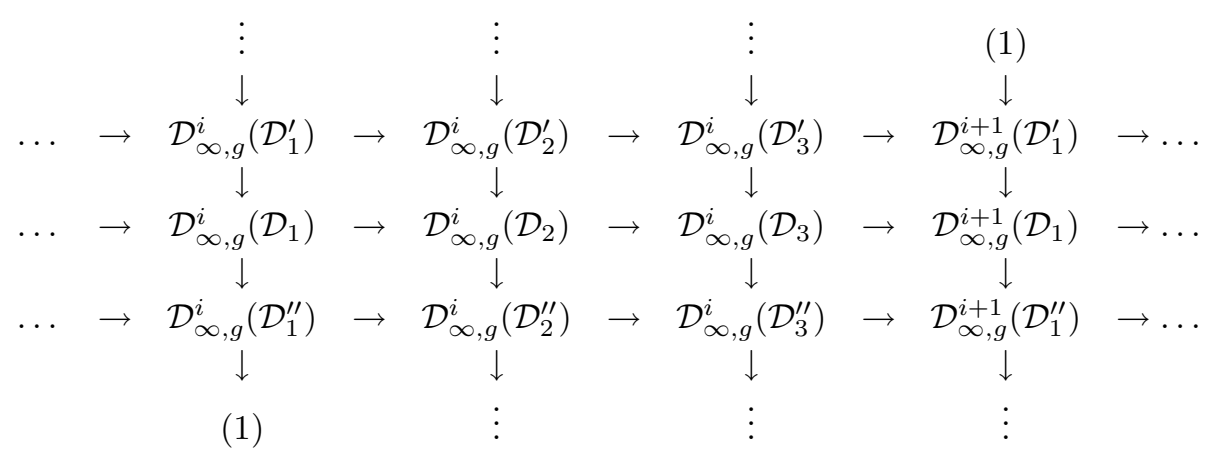

dont les colonnes (tordues) sont exactes. Par hypothèse de récurrence, la première et la dernière ligne sont exactes. En prenant le déterminant sur $\mathcal{H}(\Gamma)$, on en déduit que la deuxième ligne est exacte (voir par exemple [12]).

4.3.3. On peut introduire les notions de suites exactes $e, f$. Soit

$$
(\alpha): 0 \longrightarrow \mathcal{D}_{1} \longrightarrow \mathcal{D}_{2} \longrightarrow \mathcal{D}_{3} \longrightarrow 0
$$

une suite exacte de $(\varphi, N)$-modules. L'extension $\alpha$ est dite $f$ si la suite

$$
0 \longrightarrow \mathcal{D}_{1}^{N=0} \longrightarrow \mathcal{D}_{2}^{N=0} \longrightarrow \mathcal{D}_{3}^{N=0} \longrightarrow 0
$$


est exacte. Elle est dite $e$ si elle est $f$ et si la suite

$$
0 \longrightarrow \mathcal{U}\left(\mathcal{D}_{1} / N \mathcal{D}_{1}\right)^{\Phi=1} \longrightarrow \mathcal{U}\left(\mathcal{D}_{2} / N \mathcal{D}_{2}\right)^{\Phi=1} \longrightarrow \mathcal{U}\left(\mathcal{D}_{3} / N \mathcal{D}_{3}\right)^{\Phi=1} \longrightarrow 0
$$

est exacte.

Cette dernière condition est équivalente à l'exactitude de

$$
0 \longrightarrow\left(\mathcal{D}_{1} / N \mathcal{D}_{1}\right)^{\varphi=p^{j}} \longrightarrow\left(\mathcal{D}_{2} / N \mathcal{D}_{2}\right)^{\varphi=p^{j}} \longrightarrow\left(\mathcal{D}_{3} / N \mathcal{D}_{3}\right)^{\varphi=p^{j}} \longrightarrow 0
$$

pour tout entier $j \in \mathbb{Z}$.

On démontre facilement à l'aide de ce qui précède la proposition suivante.

4.3.4. Proposition. - Si $(\alpha)$ est une suite exacte courte de $(\varphi, N)$-modules qui est $*$ avec $*=e$ ou $f$, la suite naturelle

$$
0 \longrightarrow \mathcal{D}_{\infty, *}\left(\mathcal{D}_{1}\right) \longrightarrow \mathcal{D}_{\infty, *}\left(\mathcal{D}_{2}\right) \longrightarrow \mathcal{D}_{\infty, *}\left(\mathcal{D}_{3}\right) \longrightarrow 0
$$

est exacte. 


\section{CHAPITRE 5}

\section{EXPONENTIELLE}

\subsection{Construction d'éléments de $B_{\mathrm{dR}}$}

On fixe un système compatible de racines de l'unité $\varepsilon=\left(\zeta_{n}\right)$ avec $\zeta_{n}$ une racine de l'unité d'ordre $p^{n}$ et $\zeta_{n+1}^{p}=\zeta_{n}$. On peut voir $\varepsilon$ comme un élément de $B_{\text {cris }}$, il est noté $[\varepsilon]$. On pose $\beta_{n}=\varphi^{-n}([\varepsilon])$ dans $B_{\text {cris }}$. On pose $t=\log [\varepsilon] \in B_{\text {cris }}$. Nous utiliserons aussi l'anneau $B_{\max }$ introduit par Colmez [6, III.2] ainsi que sa norme $\|\cdot\|_{\max }$. Ils vérifient en particulier

$$
\varphi\left(B_{\text {max }}\right) \subset B_{\text {cris }} \subset B_{\text {max }}
$$

et

$$
p^{-1}\|x\|_{\max }\|y\|_{\max } \leqslant\|x y\|_{\max } \leqslant\|x\|_{\max }\|y\|_{\max }
$$

pour $x$ et $y$ appartenant à l'anneau $A_{\max }$ des éléments de $B_{\max }$ de norme $\leqslant 1$.

\subsubsection{Proposition}

1) Soit $F=\sum_{k \in \mathbb{Z}} a_{k} x^{k} \in \mathcal{H}^{[\rho, 1[}$. Alors, si $\rho_{n} \geqslant \rho$, la série $\sum_{k \in \mathbb{Z}} a_{k}\left(\beta_{n}-1\right)^{k}$ converge dans $B_{\mathrm{dR}}^{+G_{\mathbb{Q}_{p}\left(\mu_{p} \infty\right)}}$ et on note la somme $F\left(\beta_{n}-1\right)$.

$$
\text { Si } F \in \mathcal{H}^{[0,1[}, \quad F\left(\beta_{n}-1\right) \text { appartient à } B_{\text {cris }}^{+G_{\mathbb{Q}_{p}\left(\mu_{p} \infty\right)}} .
$$

2) L'application $F \mapsto F\left(\beta_{n}-1\right)$ est continue.

Démonstration [7, proposition II.5.1]. — On a

$$
\sum_{k \in \mathbb{Z}} a_{k}\left(\beta_{n}-1\right)^{k}=\sum_{k \in \mathbb{Z}} a_{k}\left(\zeta_{n}-1\right)^{k}\left(\frac{\beta_{n}-1}{\zeta_{n}-1}\right)^{k} .
$$

Les $\left(\left(\beta_{n}-1\right) /\left(\zeta_{n}-1\right)\right)^{k}$ forment une suite bornée dans $B_{\mathrm{dR}}^{+}$car l'image de $\left(\beta_{n}-1\right) /\left(\zeta_{n}-1\right)$ dans $\mathbb{C}_{p}$ est 1 . D'autre part, $\left|a_{k}\left(\zeta_{n}-1\right)^{k}\right|$ tend vers 0 dans $\mathbb{C}_{p}$ puisque la série $\sum_{k \in \mathbb{Z}} a_{k} x^{k}$ converge pour $|x|=\rho_{n}=\left|\zeta_{n}-1\right|$ lorsque $k \rightarrow \pm \infty$. En utilisant les propriétés sur les ensembles bornés énoncées dans $[\mathbf{7}, \S 5]$, on en déduit que $\left(\zeta_{n}-1\right)^{k} a_{k}\left(\left(\beta_{n}-1\right) /\left(\zeta_{n}-1\right)\right)^{k}$ tend vers 0 lorsque $k \rightarrow \pm \infty$ et la convergence 
de la série. La seconde assertion est démontrée dans [19]. Il reste à montrer que si $\left\|F_{j}\right\|_{\rho}$ tend vers 0 lorsque $j \rightarrow \infty$ (avec $\left.\rho=\left|\zeta_{n}-1\right|\right), F_{j}\left(\beta_{n}-1\right)$ tend vers 0 dans $B_{\mathrm{dR}}^{+}$. Soit $\pi_{j}$ une famille d'éléments de $\overline{\mathbb{Q}}_{p}$ tel que $\|F\|_{j} \leqslant\left|\pi_{j}\right|$ avec $\left|\pi_{j}\right|$ tendant vers 0 et posons $G_{j}=\pi_{j}^{-1} F_{j}=\sum_{k} a_{k}^{j} x^{k}$. En reprenant la démonstration de la première partie, on montre que les $a_{k}^{j}\left(\beta_{n}-1\right)^{k}$ sont bornés par rapport à $k$ et à $j$. Il en est donc de même de l'adhérence du $\mathbb{Z}_{p}$-module qu'ils engendrent. Donc les $G_{j}\left(\beta_{n}-1\right)$ sont bornés (par rapport à $j$ ). Comme la suite $\pi_{j}$ tend vers 0 dans $B_{\mathrm{dR}}^{+}$, la suite $F_{j}\left(\beta_{n}-1\right)=\pi_{j} G_{j}\left(\beta_{n}-1\right)$ tend vers 0 dans $B_{\mathrm{dR}}^{+}$.

La formule $\beta_{n}=\zeta_{n} \exp \left(t / p^{n}\right)$ permet de voir $F\left(\beta_{n}-1\right)$ comme un élément de $\mathbb{Q}_{p}\left(\mu_{p^{n}}\right) \llbracket t \rrbracket[6]$. Le lien entre $\psi$ et l'opérateur de trace $\operatorname{Tr}_{\mathbb{Q}_{p}\left(\mu_{p^{n+1}}\right) / \mathbb{Q}_{p}\left(\mu_{p^{n}}\right)}=\operatorname{Tr}_{n+1 / n}$ induit par la trace sur $\mathbb{Q}_{p}\left(\mu_{p^{n+1}}\right)$ et par l'identité sur $t$ est

$$
\psi(F)\left(\beta_{n}-1\right)=\operatorname{Tr}_{\mathbb{Q}_{p}\left(\mu_{p^{n+1}}\right) / \mathbb{Q}_{p}\left(\mu_{p^{n}}\right)}\left(F\left(\beta_{n+1}-1\right)\right) .
$$

5.1.2. Lemme. - Si $f \in \mathcal{B}_{-}$, la suite $f\left(\beta_{n}-1\right)$ est bornée dans $B_{\mathrm{dR}}$ et $p^{q n} f\left(\beta_{n}-1\right)$ tend vers 0 pour tout $q>0$.

Démonstration. - Soit $f=\sum_{k \geqslant 0} a_{k} x^{-k} \in \mathcal{B}_{-}$: on a donc $\left|a_{k}\right| \leqslant C$ et $\left|a_{k}\right| \leqslant C \rho_{n}^{k}$ pour tout entier $k, n$ assez grand et $C$ une constante. On a

$$
\sum_{k \geqslant 0} a_{k}\left(\beta_{n}-1\right)^{-k}=\sum_{r \geqslant 1} \sum_{j=0}^{p^{n}-1} \frac{a_{r p^{n}-j}}{\left(\zeta_{n}-1\right)^{r p^{n}}}\left(\beta_{n}-1\right)^{j}\left(\frac{\zeta_{n}-1}{\beta_{n}-1}\right)^{r p^{n}} .
$$

Comme $\left|a_{r p^{n}-j} /\left(\zeta_{n}-1\right)^{r p^{n}}\right| \leqslant C \rho_{n}^{-j}$ et $\left\|\left(\beta_{n}-1\right)^{j}\right\|_{\max } \leqslant \rho_{n}^{j}$, on a

$$
\left|\left(\zeta_{n}-1\right)^{r p^{n}}\right|^{-1}\left\|a_{r p^{n}-j}\left(\beta_{n}-1\right)^{j}\right\|_{\max } \leqslant C
$$

et la suite $\left(a_{r p^{n}-j} /\left(\zeta_{n}-1\right)^{r p^{n}}\right)\left(\beta_{n}-1\right)^{j}$ est bornée dans $B_{\mathrm{dR}}^{+}$. D'autre part, l'image de $\left(\zeta_{n}-1\right) /\left(\beta_{n}-1\right)$ dans $\mathbb{C}_{p}$ est égale à 1 et la suite $\left(\left(\zeta_{n}-1\right) /\left(\beta_{n}-1\right)\right)^{p^{n}}$ converge dans $B_{\mathrm{dR}}^{+}$. On en déduit que la famille des $\left(a_{r p^{n}-j} /\left(\zeta_{n}-1\right)^{r p^{n}}\right)\left(\beta_{n}-1\right)^{j} \times$ $\left(\left(\zeta_{n}-1\right) /\left(\beta_{n}-1\right)\right)^{r p^{n}}$ est bornée dans $B_{\mathrm{dR}}^{+}$de même que l'adhérence du $\mathbb{Z}_{p}$-module engendré par ces éléments. Donc, $f\left(\beta_{n}-1\right)$ est bornée dans $B_{\mathrm{dR}}^{+}$. Pour toute suite $u_{n}$ tendant vers 0 , par exemple $p^{q n}$ avec $q>0$, la suite $u_{n} f\left(\beta_{n}-1\right)$ tend vers 0 lorsque $n \rightarrow \infty$.

5.1.3. Lemme. - La suite $p^{n} \log \left(\beta_{n}-1\right)$ a une limite dans $B_{\mathrm{dR}}^{+}$qui est $\rho=\log [\varepsilon-1]$. $C^{\prime}$ est un élément de $B_{\mathrm{st}}^{+G_{\mathbb{Q}_{p}\left(\mu_{p} \infty\right)}}$ vérifiant $N \rho=1$ et $\varphi \rho=p \rho$. L'action de $G_{\infty}$ est donnée par $\tau \rho=\rho+\rho_{\tau}$ avec $\rho_{\tau}=\lim _{n} p^{n} a_{\tau}\left(\beta_{n}-1\right)$. La suite $p^{k n} \Theta_{k}\left(\beta_{n}-1\right)$ a une limite dans $B_{\mathrm{st}}^{+G_{\mathbb{Q}_{p}\left(\mu_{p} \infty\right)}}$ qui est $\rho^{k}=\log ^{k}[\varepsilon-1]$.

Rappelons que

$$
a_{\tau}=\log \frac{(1+x)^{\chi(\tau)}-1}{x}=\log \chi(\tau)+(\chi(\tau)-1) x+\cdots .
$$


Démonstration. - On peut soit le faire directement : dans $B_{\mathrm{st}}$, la suite $\left([\varepsilon]^{1 / p^{n}}-1\right)^{p^{n}}$ tend vers $[\varepsilon-1]$, soit montrer la convergence de la suite $p^{n} \log \left(\beta_{n}-1\right)$ en utilisant le lemme précédent (ce qui ne donne pas le fait que la limite appartient à $B_{\mathrm{st}}^{+}$). Comme

$$
\log \left(\beta_{n-1}-1\right)-p \log \left(\beta_{n}-1\right)=p a_{\varphi}\left(\beta_{n}-1\right),
$$

il suffit de monter que la suite $p^{n} a_{\varphi}\left(\beta_{n}-1\right)$ tend vers 0 dans $B_{\mathrm{dR}}^{+}$, ce qui est impliqué par le lemme 5.1.2. Pour les mêmes raisons, la suite $p^{n} a_{\tau}\left(\beta_{n}-1\right)$ est convergente.

Soit $H=\Theta_{k}-\log ^{k} x$; c'est un élément de $\mathcal{B}_{-}[\log x]$ de degré $\leqslant k-2$. Écrivons $H=\sum_{j=0}^{k-2} h_{j} \log ^{j} x$ avec $h_{j} \in \mathcal{B}_{-}$. On a

$$
p^{n k} H\left(\beta_{n}-1\right)=\sum_{j=0}^{k-2} p^{n} h_{j}\left(\beta_{n}-1\right) p^{n(k-1)} \log ^{j}\left(\beta_{n}-1\right) .
$$

Or $p^{n} h_{j}\left(\beta_{n}-1\right)$ tend vers 0 grâce au lemme 5.1.2, $p^{n(k-1)} \log ^{j}\left(\beta_{n}-1\right)$ tend vers 0 car $k-1>j$ et que la suite $p^{n j} \log ^{j}\left(\beta_{n}-1\right)$ converge. Donc $p^{n k} H\left(\beta_{n}-1\right)$ tend vers 0 lorsque $n \rightarrow \infty$. On en déduit le lemme.

Si $\mathcal{D}$ est un $(\varphi, N)$-module, $G \in \mathcal{B}[\log x] \otimes \mathcal{D}, G\left(\beta_{n}-1\right)$ est définie pour $n \gg 0$, même si $G([\varepsilon]-1)$ n'existe pas. Il est commode de poser $\Phi^{-n}(G([\varepsilon]-1))=(1 \otimes \varphi)^{-n} G\left(\beta_{n}-1\right)$.

5.1.4. Proposition. - Soit $G \in \mathcal{B}[\log x] \otimes \mathcal{D}$ tel que $(\psi-1 \otimes \varphi) G=\psi(\mathfrak{M})$ et $N G=\Lambda$ avec $\mathfrak{M}$ et $\Lambda \in \mathcal{U}_{0}(\mathcal{D})$. On suppose que $g=(1-\Phi) G-\mathfrak{M}$ est d'ordre $0^{-}$. La suite définie par

$$
\begin{aligned}
\Phi^{-n}(G([\varepsilon]-1))-\sum_{r=1}^{n} \Phi^{-r}(\mathfrak{M}([\varepsilon]-1)) & \\
& =(1 \otimes \varphi)^{-n} G\left(\beta_{n}-1\right)-\sum_{r=1}^{n}(1 \otimes \varphi)^{-r} \mathfrak{M}\left(\beta_{t}-1\right)
\end{aligned}
$$

pour $n \gg 0$ admet une limite $\mathcal{L}_{\mathcal{D}}(G)$ dans $B_{\mathrm{dR}}^{+G_{\mathbb{Q}_{p}\left(\mu_{p} \infty\right)}} \otimes \mathcal{D}$ qui appartient à $B_{\mathrm{st}}^{+G_{\mathbb{Q}_{p}\left(\mu_{p} \infty\right)}} \otimes \mathcal{D}$ et vérifie

$$
\begin{aligned}
\Phi \mathcal{L}_{\mathcal{D}}(G) & =\mathcal{L}_{\mathcal{D}}(G)+\mathfrak{M}([\varepsilon]-1) \\
N \mathcal{L}_{\mathcal{D}}(G) & =\Lambda([\varepsilon]-1) .
\end{aligned}
$$

De plus, si $\left(G_{0}, \Lambda\right)$ vérifie les conditions $(\mathrm{H})$,

$$
\mathcal{L}_{\mathcal{D}}\left(\widetilde{\mathcal{N}}_{\mathcal{D}}\left(G_{0}, \Lambda\right)\right)=\exp (-\rho \cdot(1 \otimes N)) \mathcal{L}_{\overline{\mathcal{D}}}\left(G_{0}\right)+\mathcal{A}(\rho \cdot N) \Lambda([\varepsilon]-1)
$$

avec

$$
\mathcal{A}(\rho \cdot N)=\sum_{i=1}^{\infty} \frac{(-1)^{i-1}}{i !} \rho^{i}(1 \otimes N)^{i-1} .
$$

Rappelons que $\overline{\mathcal{D}}$ désigne le $\varphi$-module $D$ avec opérateur de monodromie $N=0$. Démontrons d'abord un lemme. 
5.1.5. Lemme. - Soit $g \in \mathcal{B}[\log x] \otimes \mathcal{D}$ d'ordre $\leqslant 0^{-}$. La limite de $\Phi^{-n}(g([\varepsilon]-1))=$ $(1 \otimes \varphi)^{-n} g\left(\beta_{n}-1\right)$ existe dans $B_{\mathrm{dR}}$ et est nulle.

Démonstration. - Soit $g \in \mathcal{B}[\log x] \otimes \mathcal{D}, g=\sum_{k} g_{k} \log ^{k} x$ avec $g_{k} \in \mathcal{B} \otimes \mathcal{D}$ d'ordre $\leqslant(-k)^{-}$. On a

$$
(1 \otimes \varphi)^{-n} g\left(\beta_{n}-1\right)=\sum_{k} p^{-n k}(1 \otimes \varphi)^{-n} g_{k}\left(\beta_{n}-1\right)\left(p^{n} \log \left(\beta_{n}-1\right)\right)^{k} .
$$

La suite $p^{n} \log \left(\beta_{n}-1\right)$ étant convergente est bornée. La suite $p^{-n k}(1 \otimes \varphi)^{-n} g_{k}\left(\beta_{n}-1\right)$ tend vers 0 : montrons-le en décomposant $g_{k}$ en $h_{+}+h_{-}$avec $h_{+} \in \mathcal{H} \otimes \mathcal{D}$ et $h_{-} \in$ $\mathcal{B}_{-} \otimes \mathcal{D}$ d'ordre $\leqslant(-k)^{-}$. Le premier cas se déduit de l'inégalité

$$
\left\|p^{-n k}(1 \otimes \varphi)^{-n} h_{+}\left(\beta_{n}-1\right)\right\|_{\max } \leqslant C\left\|p^{-n k}(1 \otimes \varphi)^{-n} h_{+}\right\|_{\rho_{n}} .
$$

Dans le second cas, la démonstration du lemme 3.3.6 implique que si $\mathcal{D}^{\prime}$ est le $\varphi$ support de $h_{-}$(c'est-à-dire le plus petit sous- $\varphi$-module de $\mathcal{D}$ tel que $h_{-} \in \mathcal{B} \otimes \mathcal{D}^{\prime}$ ), $\left|\varphi^{-1}\right|_{\mathcal{D}^{\prime}}<p^{-k}$. D'après le lemme 5.1.2, $h_{-}\left(\beta_{n}-1\right)$ est bornée. On en conclut que $p^{-n k}(1 \otimes \varphi)^{-n} h_{-}\left(\beta_{n}-1\right)$ tend vers 0 .

Passons à la démonstration proprement dite de la proposition 5.1.4. Posons

$$
V_{n}=V_{n}(G)=\Phi^{-n}(G([\varepsilon]-1))-\sum_{r=1}^{n} \Phi^{-r}(\mathfrak{M}([\varepsilon]-1)) .
$$

On a pour $n \gg 0$

$$
\begin{aligned}
V_{n}-V_{n-1} & =\Phi^{-n}(1-\Phi)(G([\varepsilon]-1))-\Phi^{-n}(\mathfrak{M}([\varepsilon]-1)) \\
& =(1 \otimes \varphi)^{-n} g\left(\beta_{n}-1\right) .
\end{aligned}
$$

Lorsque $g$ est d'ordre $\leqslant 0^{-}$, la suite $V_{n}$ a donc une limite que l'on note $\mathcal{L}_{\mathcal{D}}(G)$. Il est clair que $\mathcal{L}_{\mathcal{D}}(G)$ appartient à $B_{\mathrm{dR}}^{+G_{\mathbb{Q}}\left(\mu_{p} \infty\right)}$. Pour démontrer que $\mathcal{L}_{\mathcal{D}}(G)$ appartient à $B_{\text {st }}$, nous allons utiliser $\widetilde{\mathcal{N}}_{\mathcal{D}}$. D'après le $\S 3.3$, il existe $G_{0} \in \mathcal{B} \otimes \mathcal{D}$ tel que

$$
G=\widetilde{\mathcal{N}}_{\mathcal{D}}\left(G_{0}, \Lambda\right), \quad(\psi-1 \otimes \varphi) G_{0}=\psi(\mathfrak{M})
$$

et tel que

$$
g_{0}=(1-\Phi) G_{0}-\mathfrak{M}
$$

soit d'ordre $\leqslant 0^{-}$. En fait $G_{0} \in \mathcal{H} \otimes \mathcal{D}$. En effet, si on décompose $G_{0}$ sur $\mathcal{B}_{-}+\mathcal{B}_{-}$, $(\psi-1 \otimes \varphi) G_{0,-}=0$ et $(1-\Phi) G_{0,-}$ est d'ordre $\leqslant 0^{-}$. Le lemme 3.3 .6 implique que $G_{0,-}$ est nul. En utilisant ce qui a été montré de la proposition pour un $\varphi$-module vérifiant $N=0$, les $\bar{V}_{n}=\Phi^{-n}\left(G_{0}([\varepsilon]-1)\right)-\sum_{r=1}^{n} \Phi^{-r}(\mathfrak{M}([\varepsilon]-1))$ convergent vers un élément de $B_{\mathrm{dR}}^{+G_{\mathbb{Q}_{p}\left(\mu_{p} \infty\right)}}$. Comme d'autre part $G_{0} \in \mathcal{H} \otimes \mathcal{D}$, ils appartiennent à $B_{\text {cris }}^{+G_{\mathbb{Q}_{p}\left(\mu_{p} \infty\right)}}$ de même que leur limite que l'on note $\mathcal{L}_{\overline{\mathcal{D}}}\left(G_{0}\right)$. On a $\Phi\left(\bar{V}_{n}\right)-\bar{V}_{n-1}=\mathfrak{M}([\varepsilon]-1)$, d'où

$$
\Phi\left(\mathcal{L}_{\overline{\mathcal{D}}}\left(G_{0}\right)\right)=\mathcal{L}_{\overline{\mathcal{D}}}\left(G_{0}\right)+\mathfrak{M}([\varepsilon]-1) .
$$


Cherchons le lien existant entre $\mathcal{L}_{\overline{\mathcal{D}}}\left(G_{0}\right)$ et $\mathcal{L}_{\mathcal{D}}(G)=\mathcal{L}_{\mathcal{D}}\left(\widetilde{\mathcal{N}}_{\mathcal{D}}\left(G_{0}, \Lambda\right)\right)$. Ce dernier est la limite lorsque $k$ et $n$ tendent vers l'infini de (on pose $\Psi=(1 \otimes \varphi)^{-1} \psi$ )

$$
\begin{aligned}
Z_{n, k}=\Phi^{-n}\left(\Psi^{k}\left(\Theta\left(G_{0}\right)\right)\right. & \left.([\varepsilon]-1)-\sum_{r=1}^{k} \Psi^{r}(\mathfrak{M})([\varepsilon]-1)\right) \\
& -\Phi^{-n}\left(\Psi^{k}\left(\int \Theta(\Lambda)\right)([\varepsilon]-1)\right)-\sum_{r=1}^{n} \Phi^{-r}(\mathfrak{M}([\varepsilon]-1)) .
\end{aligned}
$$

On a

$$
\Phi^{-n}\left(\Psi^{k}(H)([\varepsilon]-1)\right)=\Phi^{-n-k}(H([\varepsilon]-1))
$$

Autrement dit, $\Psi=(1 \otimes \varphi)^{-1} \psi$ devient un inverse à droite et à gauche de $\Phi$. En effet,

$$
\psi(H)\left(\beta_{n}-1\right)=(\psi \circ(\varphi \otimes 1))(H)\left(\beta_{n+1}-1\right)=H\left(\beta_{n+1}-1\right)
$$

et

$$
\begin{aligned}
\Phi^{-n}\left(\Psi^{k}(H)([\varepsilon]-1)\right) & =(1 \otimes \varphi)^{-n-k} \psi^{k}(H)\left(\beta_{n}-1\right) \\
& =(1 \otimes \varphi)^{-n-k} H\left(\beta_{n+k}-1\right) \\
& =\Phi^{-n-k}(H([\varepsilon]-1)) .
\end{aligned}
$$

On pose $m=n+k$. On a alors

$$
\begin{aligned}
Z_{n, k}= & \Phi^{-m}\left(\Theta\left(G_{0}\right)([\varepsilon]-1)\right)-\sum_{r=1}^{m} \Phi^{-r}(\mathfrak{M}([\varepsilon]-1))+\Phi^{-m}\left(\int \Theta(\Lambda)([\varepsilon]-1)\right) \\
= & \Phi^{-m}\left(\Theta\left(G_{0}\right)([\varepsilon]-1)\right)-\sum_{r=1}^{m} \Phi^{-m}\left(\Theta\left(\Phi^{m-r} \mathfrak{M}\right)([\varepsilon]-1)\right) \\
& \quad+\Phi^{-m}\left(\sum_{r=1}^{m}\left(\Theta\left(\Phi^{m-r} \mathfrak{M}\right)([\varepsilon]-1)-\Phi^{r}(\mathfrak{M}([\varepsilon]-1))\right)+\int \Theta(\Lambda)([\varepsilon]-1)\right) .
\end{aligned}
$$

On a

$$
\begin{aligned}
& \Phi^{-m}\left(\Theta\left(G_{0}\right)([\varepsilon]-1)\right)=\Phi^{-m}\left(\sum_{j} \frac{(-1)^{j}}{j !} \Theta_{j}(1 \otimes N)^{j} G_{0}([\varepsilon]-1)\right) \\
& =\sum_{j} \frac{(-1)^{j}}{j !} p^{m j} \Phi^{-m}\left(\Theta_{j}([\varepsilon]-1)\right)(1 \otimes N)^{j} \Phi^{-m}\left(G_{0}([\varepsilon]-1)\right) .
\end{aligned}
$$

En faisant de même sur le second morceau, on obtient en passant à la limite que

$$
\left.\Phi^{-m}\left(\Theta\left(G_{0}\right)([\varepsilon]-1)-\sum_{r=0}^{m-1} \Phi^{r} \Theta(\mathfrak{M})([\varepsilon]-1)\right)\right)
$$


tend vers $\exp (-\rho \cdot(1 \otimes N))\left(\mathcal{L}_{\overline{\mathcal{D}}}\left(G_{0}\right)\right)$. Le terme suivant à étudier est

$$
\begin{aligned}
\Phi^{-m} & \left.\left(\sum_{r=1}^{m} \Theta\left(\Phi^{m-r} \mathfrak{M}\right)([\varepsilon]-1)-\Phi^{r} \mathfrak{M}([\varepsilon]-1)\right)+\int \Theta(\Lambda)([\varepsilon]-1)\right) \\
& =\sum_{r=0}^{m-1} \Phi^{-m}\left(\int \Theta\left((p \Phi)^{r}(1-p \Phi) \Lambda([\varepsilon]-1)\right)+\Phi^{-m} \int \Theta(\Lambda)([\varepsilon]-1)\right.
\end{aligned}
$$

en utilisant $N \mathfrak{M}=(1-p \Phi) \Lambda$

$$
=\Phi^{-m}\left(\int \Theta\right)([\varepsilon]-1) \cdot \Lambda([\varepsilon]-1) .
$$

On en déduit que

$$
\mathcal{L}_{\mathcal{D}}\left(\mathcal{N}_{\mathcal{D}}\left(G_{0}, \Lambda\right)\right)=\exp (-\rho \cdot(1 \otimes N)) \mathcal{L}_{\overline{\mathcal{D}}}\left(G_{0}\right)+\mathcal{A}(\rho \cdot N) \Lambda([\varepsilon]-1) .
$$

En particulier si $G$ est comme dans la proposition 5.1.4, $\mathcal{L}_{\mathcal{D}}(G)$ appartient à $B_{\text {st }}^{+G_{\mathbb{Q}_{p}\left(\mu_{p} \infty\right)}} \otimes \mathcal{D}$. On a alors

$$
\begin{aligned}
N \mathcal{L}_{\mathcal{D}}(G) & =N \sum_{i=1}^{\infty} \frac{(-1)}{i !} \rho^{i}(1 \otimes N)^{i-1} \Lambda([\varepsilon]-1)=\Lambda([\varepsilon]-1) \\
\Phi \mathcal{L}_{\mathcal{D}}(G) & =\mathcal{L}_{\mathcal{D}}(G)+\mathfrak{M}([\varepsilon]-1)
\end{aligned}
$$

5.1.6. Notons $\lambda_{k, n}$ l'application de $B_{\mathrm{dR}}^{G_{\mathrm{Q}_{p}\left(\mu_{p} \infty\right)}} \otimes \mathcal{D}$ dans $\mathbb{Q}_{p}\left(\mu_{p^{n}}\right) \otimes \mathcal{D}$ induite par

$$
B_{\mathrm{dR}}^{G_{\mathbb{Q}_{p}\left(\mu_{p} \infty\right)}} \longrightarrow B_{\mathrm{dR}}^{G_{\mathbb{Q}_{p}\left(\mu_{p} \infty\right)}} /\left(\chi(\gamma)^{-k p^{n}} \gamma^{p^{n}}-1\right) \cong \mathbb{Q}_{p}\left(\mu_{p^{n}}\right)
$$

(voir $[\mathbf{2 5}, \S 4.1 .2]$ pour la normalisation : pour $k=0$ et $b \in \mathbb{Q}_{p}\left(\mu_{p^{n}}\right)$, on a $\lambda_{k, n}(b)=b$ ). On a $\lambda_{k, n}(\tau x)=\chi(\tau)^{k} \lambda_{k, n}(x)$. Posons aussi $T_{n}(x)=p^{-n} \sum_{k} \lambda_{k, n}(x) t^{k}$. La proposition suivante répond à une question de Colmez.

5.1.7. Proposition. - Soit $G$ comme dans la proposition 5.1.4, c'est-à-dire vérifiant les conditions $(\mathrm{K})$. On a pour $n$ assez grand et pour $k \geqslant 0$

$\lambda_{k, n}\left(\mathcal{L}_{\mathcal{D}}(G)\right)=\frac{p^{-n k}(1 \otimes \varphi)^{-n} D^{k}(G)\left(\zeta_{n}-1\right)-\sum_{r=1}^{n} p^{-r k}(1 \otimes \varphi)^{-r} D^{k}(\mathfrak{M})\left(\zeta_{r}-1\right)}{k !}$

et

$$
\begin{aligned}
p^{n} T_{n}\left(\mathcal{L}_{\mathcal{D}}(G)\right) & =(1 \otimes \varphi)^{-n} G\left(\beta_{n}-1\right)-\sum_{r=1}^{n}(1 \otimes \varphi)^{-r} \mathfrak{M}\left(\beta_{r}-1\right) \\
& =\Phi^{-n} G([\varepsilon]-1)-\sum_{r=1}^{n} \Phi^{-r} \mathfrak{M}([\varepsilon]-1) .
\end{aligned}
$$

Remarquons que $D^{k}(\mathfrak{M})\left(\zeta_{r}-1\right)=D^{k}(\mathfrak{M})(0)$ et que la formule peut donc s'écrire

$$
\lambda_{k, n}\left(\mathcal{L}_{\mathcal{D}}(G)\right)=\frac{p^{-n k}(1 \otimes \varphi)^{-n} D^{k}(G)\left(\zeta_{n}-1\right)-\sum_{r=1}^{n} p^{-r k}(1 \otimes \varphi)^{-r} \mathfrak{M}_{k}}{k !}
$$

avec $\mathfrak{M}=\sum_{k} \mathfrak{M}_{k} \mathbf{t}_{k}$. 
Démonstration. - Par continuité de $\lambda_{k, n}, \lambda_{k, n}\left(\mathcal{L}_{\mathcal{D}}(G)\right)$ est la limite lorsque $m \rightarrow \infty$ de

$$
\lambda_{k, n}\left((1 \otimes \varphi)^{-n-m} G\left(\beta_{n+m}-1\right)-\sum_{r=1}^{n+m}(1 \otimes \varphi)^{-r} \mathfrak{M}\left(\beta_{r}-1\right)\right) .
$$

Si $H \in \mathcal{B}[\log x] \otimes \mathcal{D}$, on a pour $n \gg 0$ et pour $k \geqslant 0$ comme dans $[\mathbf{2 5}, \S 4.1 .2]$ en remplaçant $m$ par $m+n$

$$
\lambda_{k, n}\left(H\left(\beta_{m+n}-1\right)\right)=\frac{p^{-m} \operatorname{Tr}_{m+n / n}\left(D^{k}(H)\left(\zeta_{m+n}-1\right)\right)}{p^{(m+n) k} k !}
$$

et pour $r \leqslant n$,

$$
\lambda_{k, n}\left(H\left(\beta_{r}-1\right)\right)=\frac{\left.D^{k}(H)\left(\zeta_{r}-1\right)\right)}{p^{r k} k !} .
$$

Posons $\Psi=(1 \otimes \varphi)^{-1} \psi$. L'équation $\Psi\left(D^{k}(G)\right)=p^{k} D^{k}(G)+\Psi\left(D^{k}(\mathfrak{M})\right)$ implique pour tout entier $m$

$$
\Psi^{m}\left(D^{k}(G)\right)=p^{k m} D^{k}(G)+\sum_{r=1}^{m} p^{k(m-r)} \Psi^{r}\left(D^{k}(\mathfrak{M})\right) .
$$

On en déduit que

$$
\begin{aligned}
(1 \otimes \varphi)^{-m} p^{-m} \operatorname{Tr}_{m+n / n}\left(D^{k}(G)\left(\zeta_{n+m}-1\right)\right) \\
\quad=p^{k m} D^{k}(G)\left(\zeta_{n}-1\right)+\sum_{r=1}^{m} p^{k(m-r)}(1 \otimes \varphi)^{-r} p^{-r} \operatorname{Tr}_{r+n / n}\left(D^{k}(\mathfrak{M})\left(\zeta_{n+r}-1\right)\right) .
\end{aligned}
$$

Donc $\lambda_{k, n}\left(\mathcal{L}_{\mathcal{D}}(G)\right)$ est la limite lorsque $m \rightarrow \infty$ de

$$
\begin{gathered}
\frac{p^{-m}(1 \otimes \varphi)^{-n-m} \operatorname{Tr}_{m+n / n}\left(D^{k}(G)\left(\zeta_{m+n}-1\right)\right)}{p^{(n+m) k} k !} \\
-\sum_{r=1}^{m} \frac{(1 \otimes \varphi)^{-r-n} p^{-r} \operatorname{Tr}_{r+n / n}\left(D^{k}(\mathfrak{M})\left(\zeta_{n+r}-1\right)\right)}{p^{(n+r) k} k !} \\
-\sum_{r=1}^{n} \frac{\left.(1 \otimes \varphi)^{-r} D^{k}(\mathfrak{M})\left(\zeta_{r}-1\right)\right)}{p^{n r} k !} \\
=\frac{p^{-n k}(1 \otimes \varphi)^{-n} D^{k}(G)\left(\zeta_{n}-1\right)-\sum_{r=1}^{n} p^{-n r}(1 \otimes \varphi)^{-r} D^{k}(\mathfrak{M})\left(\zeta_{r}-1\right)}{k !} .
\end{gathered}
$$

On déduit la formule concernant $T_{n}$ de la remarque et de la formule de Taylor

$$
\begin{aligned}
H\left(\beta_{n}-1\right) & =\sum_{k=0}^{\infty} D^{k}(H)\left(\zeta_{n}-1\right) \frac{\left(\log \beta_{n}\right)^{k}}{k !} \\
& =\sum_{k=0}^{\infty} D^{k}(H)\left(\zeta_{n}-1\right) \frac{t^{k}}{p^{n k} k !}
\end{aligned}
$$

(rappelons que $\beta_{n}=\zeta_{n} \exp \left(t / p^{n}\right)$ ). 
5.1.8. On peut utiliser les résultats qui précèdent pour démontrer la généralisation suivante du théorème de Coleman-Colmez (voir l'appendice de [25] pour une présentation analogue). Nous ne nous en servirons pas dans la suite.

Voyons $\mathcal{U}_{0}(\mathcal{D})$ comme plongé dans $B_{\text {cris }} \otimes \mathcal{D}: \mathcal{U}_{0}(\mathcal{D})=\oplus_{i \geqslant 0} \mathcal{D} t^{j}$ en envoyant $\mathbf{t}_{j}$ sur $t^{j} / j$ ! avec $t=\log [\varepsilon]$. Pour distinguer, on notera $\Lambda_{\text {cris }}=\Lambda([\varepsilon]-1)$ l'image de $\Lambda$ dans $B_{\text {cris }} \otimes \mathcal{D}$. On note $\mathcal{D}_{\infty, g}(\mathcal{D})_{0}^{(0)}$ le sous- $\mathbb{Z}_{p} \llbracket G_{\infty} \rrbracket$-module de $\mathcal{D}_{\infty, g}(\mathcal{D})$ formé des éléments $g$ d'ordre $\leqslant 0^{-}$et dont l'image par $\mathcal{R}_{\mathcal{D}}$ dans $\mathcal{C}_{\infty}(\mathcal{D})$ appartient à $\mathcal{C}_{\infty, \geqslant 0}(\mathcal{D})$ obtenu à partir du complexe $\mathfrak{C}\left(\mathcal{U}_{0}(\mathcal{D})\right)$.

5.1.9. Proposition. - On a la suite exacte de $G_{\infty}$-modules

$$
\begin{aligned}
0 \longrightarrow \mathcal{U}_{0}(\mathcal{D})_{\mathrm{cris}}^{N=0, \Phi=1} \longrightarrow\left(B_{\mathrm{st}}^{+G_{\mathbb{Q}_{p}\left(\mu_{p} \infty\right)}} \otimes \mathcal{D}\right)^{\Phi=1, N=0} \\
\quad \longrightarrow \mathcal{D}_{\infty, g}(\mathcal{D})_{0}^{(0)} \longrightarrow \mathcal{C}_{\infty, \geqslant 0}(\mathcal{D}) \longrightarrow 0
\end{aligned}
$$

Démonstration. — L'application « réciproque » $\mathcal{C}_{\mathcal{D}}^{-1}$ est l'application

$$
\mathcal{D}_{\infty, e}(\mathcal{D}) \rightarrow\left(B_{\text {st }}^{+G_{\mathbb{Q}_{p}\left(\mu_{p} \infty\right)}} \otimes \mathcal{D}\right)^{\Phi=1, N=0}
$$

définie de la manière suivante : on écrit $(1-\Phi) G=g$ et on prend la limite $\mathcal{L}_{\mathcal{D}}(G)$ de $(1 \otimes \varphi)^{-n} G\left(\beta_{n}-1\right)$. Si l'on en prend la classe modulo $\oplus_{i \geqslant 0} \mathcal{D}^{N=0, \varphi=p^{-j}} t^{j}$, cela ne dépend que de $g=(1-\Phi) G$ et non du choix de $G$.

La construction de l'application dans le sens direct est une conséquence du théorème de Colmez dans le cas où $\mathcal{D}$ est un $\varphi$-module et des constructions faites au $\S 3.2$. Soit $\beta \in\left(B_{\mathrm{st}}^{+G_{\mathbb{Q}_{p}\left(\mu_{p} \infty\right)}} \otimes \mathcal{D}\right)^{\Phi=1, N=0}$. Il s'écrit de manière unique sous la forme

$$
\beta=\sum_{j=0}^{\infty} \frac{(-1)^{j}}{j !} \rho^{j}(1 \otimes N)^{j} \beta_{0}
$$

avec $\beta_{0} \in\left(B_{\text {cris }}^{+G_{\mathbb{Q}_{p}\left(\mu_{p} \infty\right)}} \otimes \mathcal{D}\right)^{\Phi=1}$. Soit $F_{\beta}$ son image par l'homomorphisme de Colmez $\mathcal{C}_{\overline{\mathcal{D}}}$ dans $\mathcal{D}_{\infty, e}(\overline{\mathcal{D}})$ avec $\overline{\mathcal{D}}$ le $\varphi$-module $\mathcal{D}$. On prend alors

$$
\mathcal{C}_{\mathcal{D}}(\beta)=\mathcal{N}_{\mathcal{D}}\left(F_{\beta}, 0\right) \in \mathcal{D}_{\infty, e}(\mathcal{D}) .
$$

Il est facile de déduire des divers résultats de ce texte que la suite est exacte et que $\mathcal{C}_{\mathcal{D}}$ et $\mathcal{C}_{\mathcal{D}}^{-1}$ sont bien «inverses » l'une de l'autre.

On peut facilement généraliser ceci à $\mathcal{D}_{\infty, g}(\mathcal{D})$, compte tenu de ce qui a été fait. Considérons le complexe $\mathfrak{C}_{\text {cris, } g}(\mathcal{D})$ en degrés 0,1 et 2

$$
\begin{array}{clrl}
\mathcal{U}_{0}(\mathcal{D})_{\mathrm{cris}} & \rightarrow \mathcal{U}_{0}(\mathcal{D})_{\mathrm{cris}} \times \mathcal{U}_{0}(\mathcal{D})_{\mathrm{cris}} \times B_{\mathrm{st}}^{+G_{\mathbb{Q}_{p}\left(\mu_{p} \infty\right)}} \otimes \mathcal{D} & \rightarrow & \mathcal{U}_{0}(\mathcal{D})_{\mathrm{cris}} \times \mathcal{U}_{0}(\mathcal{D})_{\mathrm{cris}} \\
u & (N u,(1-\Phi) u, u) & & \\
& (\Lambda, \mathfrak{M}, x) & \mapsto(N x-\Lambda,(1-\Phi) x-\mathfrak{M})
\end{array}
$$

et notons $\left(B_{\mathrm{st}}^{+G_{\mathbb{Q}_{p}\left(\mu_{p} \infty\right)}} \otimes \mathcal{D}\right)_{g}$ le premier groupe de cohomologie. On construit une application

$$
\mathcal{D}_{\infty, g}(\mathcal{D})_{0}^{(0)} \longrightarrow\left(B_{\mathrm{st}}^{+G_{\mathbb{Q}_{p}\left(\mu_{p} \infty\right)}} \otimes \mathcal{D}\right)_{g}
$$


de la manière suivante. Soit $g \in \mathcal{D}_{\infty, g}(\mathcal{D})$ et $G$ une solution complète de l'équation $(1-\Phi) G=g$ : on a donc en fait $(1-\Phi) G=g+\mathfrak{M}(G)$. Posons $N G=\Lambda(G)$. La classe de $\left(\Lambda(G)_{\text {cris }}, \mathfrak{M}(G)_{\text {cris }}, \mathcal{L}_{\mathcal{D}}(G)\right)$ dans $\left(B_{\text {st }}^{+G_{Q_{p}\left(\mu_{p} \infty\right)}} \otimes \mathcal{D}\right)_{g}$ ne dépend pas du choix de $G$.

5.1.10. Proposition. - Soit $\mathcal{D}$ un $(\varphi, N)$-module. Alors, l'application

$$
\mathcal{L}_{\mathcal{D}}: \mathcal{D}_{\infty, g}(\mathcal{D})_{\geqslant 0}^{(0)} \longrightarrow\left(B_{\mathrm{st}}^{+G_{\mathbb{Q}_{p}\left(\mu_{p} \infty\right)}} \otimes \mathcal{D}\right)_{g}
$$

est un isomorphisme de $G_{\infty}$-modules et

$$
\begin{aligned}
p^{n} T_{n}\left(\mathcal{L}_{\mathcal{D}}(G)\right) & =(1 \otimes \varphi)^{-n} G\left(\beta_{n}-1\right)-\sum_{r=1}^{n}(1 \otimes \varphi)^{-r} \mathfrak{M}(G)\left(\beta_{r}-1\right) \\
& =\Phi^{-n}(G([\varepsilon]-1))-\sum_{r=1}^{n} \Phi^{-r}(\mathfrak{M}(G)([\varepsilon]-1)) .
\end{aligned}
$$

\subsection{Théorème-définition de l'exponentielle}

5.2.1. Soit $K$ une extension finie non ramifiée de $\mathbb{Q}_{p}$ et soit $V$ une représentation $p$-adique semi-stable de $G_{K}$. On pose $K_{n}=K\left(\mu_{p^{n}}\right)=K \mathbb{Q}_{p}\left(\mu_{p^{n}}\right)$. La représentation induite $\operatorname{Ind}_{K / \mathbb{Q}_{p}}(V)$ de $V$ à $G_{\mathbb{Q}_{p}}$ est une représentation $p$-adique semi-stable de $G_{\mathbb{Q}_{p}}$. Le $\mathbb{Q}_{p}$-espace vectoriel $\mathbf{D}_{p}(V)=\mathbf{D}_{p}\left(\operatorname{Ind}_{K / \mathbb{Q}_{p}}(V)\right)$ est muni d'une structure naturelle de $K$-espace vectoriel. D'autre part, par le lemme de Shapiro, il y a un isomorphisme naturel entre $H^{1}\left(K_{n}, V\right)$ et $H^{1}\left(\mathbb{Q}_{p}\left(\mu_{p^{n}}\right), \operatorname{Ind}_{K / \mathbb{Q}_{p}}(V)\right)$ et entre $H^{1}\left(K_{n}, T\right)$ et $H^{1}\left(\mathbb{Q}_{p}\left(\mu_{p^{n}}\right), \operatorname{Ind}_{K / \mathbb{Q}_{p}}(T)\right)$ si $T$ est un réseau de $V$ stable par $G_{K}$. On pose

$$
\mathcal{D}_{\infty}^{\text {gros }}(V)=\mathcal{D}_{\infty}^{\text {gros }}\left(\mathbf{D}_{p}(V)\right), \widetilde{\mathcal{D}}_{\infty}(V)=\widetilde{\mathcal{D}}_{\infty}\left(\mathbf{D}_{p}(V)\right)
$$

et pour $* \in\{e, f, g\}, \mathcal{D}_{\infty, *}(V)=\mathcal{D}_{\infty, *}\left(\mathbf{D}_{p}(V)\right)$.

Pour $g \in \mathcal{D}_{\infty, g}(V)$, nous appellerons abusivement solution (complète) de l'équation $(1-\Phi) G=g$ une famille $\left(G_{r}\right)_{r \in \mathbb{Z}}$ telle que

1) $G_{r} \in \mathcal{B}[\log x] \otimes \mathbf{D}_{p}(V)$ et $D\left(G_{r}\right)=G_{r+1}$

2) pour $r \gg 0,\left(1-p^{r} \Phi\right) G_{r}=D^{r}(g)$

Si $g \in \mathcal{D}_{\infty, f}(V)$, on peut prendre les $G_{s}$ dans $\left(\mathcal{B}[\log x] \otimes \mathbf{D}_{p}(V)\right)^{N=0}$. Si $g \in \mathcal{D}_{\infty, e}(V)$, on peut prendre les $G_{s} \in\left(\mathcal{B}[\log x] \otimes \mathbf{D}_{p}(V)\right)^{N=0, \psi=0}$ et on a alors $\left(1-p^{s} \Phi\right) G_{s}=D^{s}(g)$, mais ce n'est pas essentiel.

Si $G$ est une solution complète de $(1-\Phi) G=g$, on note $D^{r}(G)=G_{r}$. On a les relations « formelles » suivantes

$$
\begin{aligned}
(1-\Phi) G & =g+\mathfrak{M}(G) \\
N G & =\Lambda(G)
\end{aligned}
$$


avec $\mathfrak{M}(G)$ et $\Lambda(G) \in \mathcal{U}\left(\mathbf{D}_{p}(V)\right)$, ce qui signifie concrètement que pour tout entier $r \in \mathbb{Z}$

$$
\begin{aligned}
(1-\Phi) D^{r}(G) & =D^{r}(g)+D^{r}(\mathfrak{M}(G)) \\
N D^{r}(G) & =D^{r}(\Lambda(G))
\end{aligned}
$$

avec $D^{r}: \mathcal{U}\left(\mathbf{D}_{p}(V)\right) \rightarrow \mathcal{H} \otimes \mathbf{D}_{p}(V)$ l'application

$$
D^{r}\left(\sum \mathbf{t}^{j} b_{j}\right)=\sum_{j \geqslant r} \frac{\log ^{j-r}(1+x)}{(j-r) !} b_{j} .
$$

5.2.2. Définition. - Soit $g \in \mathcal{D}_{\infty, g}(V)$ et $G$ une solution (complète) de l'équation $(1-\Phi) G=g$. On pose $H=\left(\Lambda^{[1]}, \mathfrak{M}, G\right)$ où $\Lambda^{[1]}$ est $\Lambda$ vu comme appartenant à $\mathcal{U}\left(\mathbf{D}_{p}(V)[1]\right)$, ce qui pratiquement veut dire que

$$
(1 \otimes \varphi)\left(\Lambda^{[1]}\right)=p(1 \otimes \varphi) \Lambda .
$$

En tant que $\mathbb{Q}_{p}$-espace vectoriel, $\mathbf{D}_{p}(V)$ et $\mathbf{D}_{p}(V(k))$ sont égaux et l'homomorphisme de Frobenius de $\mathbf{D}_{p}(V(k))$ est $p^{-k}$ fois celui de $\mathbf{D}_{p}(V)$. Nous ferons cependant de temps en temps intervenir la base canonique $e_{-k}$ de $\mathbb{Q}_{p}[-k]$ (voir $[\mathbf{2 5}, \S 3.1 .1]$ pour les détails) qui est simplement 1 avec l'action $\varphi e_{-k}=p^{-k} e_{-k}$. Définissons

$$
\Xi_{n, k}(G)=p^{n(k-1)}(1 \otimes \varphi)^{-n} D^{-k}(H)\left(\zeta_{n}-1\right)
$$

vu comme un élément de $\mathcal{C}_{\text {st }}\left(V(k), K_{n}\right)$ avec $\mathcal{C}_{\text {st }}\left(V(k), K_{n}\right)$ le $H^{1}$ du complexe $\mathfrak{C}_{\mathrm{st}}\left(V(k), K_{n}\right)$ (en degré $\left.0,1,2\right)$

$$
\begin{aligned}
& \mathbf{D}_{p}(V(k)) \quad \longrightarrow \quad \mathbf{D}_{p}(V(k)) \oplus \mathbf{D}_{p}(V(k)) \oplus t_{V(k)}\left(K_{n}\right) \quad \longrightarrow \quad \mathbf{D}_{p}(V(k)) \\
& \nu \quad \longmapsto \quad\left(N \nu,\left(1-p^{-k} \varphi\right) \nu, \bar{\nu}\right) \\
& (\lambda, \mu, z) \quad \longmapsto N \mu-\left(1-p^{-k+1} \varphi\right) \lambda
\end{aligned}
$$

où

$$
\begin{aligned}
t_{V(k)}\left(K_{n}\right) & =K_{n} \otimes_{K}\left(\mathbf{D}_{\mathrm{dR}}(V(k)) / \mathrm{Fil}^{0} \mathbf{D}_{\mathrm{dR}}(V(k))\right) \\
& =K_{n} \otimes_{K}\left(\mathbf{D}_{\mathrm{dR}}(V) / \mathrm{Fil}^{k} \mathbf{D}_{\mathrm{dR}}(V)\right) \\
& =\mathbb{Q}\left(\mu_{p^{n}}\right) \otimes\left(\mathbf{D}_{\mathrm{dR}}(V) / \mathrm{Fil}^{k} \mathbf{D}_{\mathrm{dR}}(V)\right)
\end{aligned}
$$

(comme $V$ est supposé semi-stable et $K / \mathbb{Q}_{p}$ non ramifié, $\mathbf{D}_{\mathrm{dR}}(V)=\mathbf{D}_{p}(V)$ ). Remarquons que, pour $\mathcal{S} \in \mathcal{U}_{0}(\mathcal{D}), D^{-k}(\mathcal{S})\left(\zeta_{n}-1\right)=D^{-k}(\mathcal{S})(0)$ est un élément de $\mathbf{D}_{p}(V)$.

L'élément $p^{n} \Xi_{n, k}(G)$ peut aussi s'écrire

$$
\begin{aligned}
& p^{n k}(1 \otimes \varphi)^{-n}\left(p^{-n} D^{-k}(\Lambda), D^{-k}(\mathfrak{M}), D^{-k}(G)\right)\left(\zeta_{n}-1\right) \\
& \quad=(1 \otimes \varphi)^{-n}\left(p^{-n} D^{-k}(\Lambda)(0), D^{-k}(\mathfrak{M})(0), D^{-k}(G)\left(\zeta_{n}-1\right) \otimes e_{-k}\right)
\end{aligned}
$$

avec $\Lambda=\Lambda(G)$ et $\mathfrak{M}=\mathfrak{M}(G)$.

Bloch et Kato définissent dans [2] une application exponentielle

$$
\exp _{V(k)}: \mathcal{C}_{\mathrm{st}}\left(V(k), K_{n}\right) \rightarrow H^{1}\left(K_{n}, V(k)\right)
$$


dont l'image est par définition $H_{g}^{1}\left(K_{n}, V(k)\right)$. Plus précisément, $H_{g}^{1}\left(K_{n}, V(k)\right)$ est défini dans [2] comme le noyau de $H^{1}\left(K_{n}, V(k)\right) \rightarrow H^{1}\left(K_{n}, B_{\mathrm{dR}} \otimes V(k)\right)$. L'égalité avec le $\mathbb{Q}_{p}$-espace vectoriel que nous définissons ici a été montrée par Hyodo (non publié) et Nekováŕ ([15], voir aussi [12]).

Soit $T$ un réseau de $V$ stable par $G_{K}$. Soit $Z_{\infty}^{1}(K, T)$ la limite projective des $H^{1}\left(K_{n}, T\right)$ pour les applications de corestriction et $Z_{\infty}^{1}(K, V)=\mathbb{Q}_{p} \otimes Z_{\infty}^{1}(K, T)$. Notons $\pi_{n, k}: Z_{\infty}^{1}(K, T) \rightarrow H^{1}\left(K_{n}, V(k)\right)$ le composé de l'opérateur de twist induit par $V \rightarrow V(k)$ et de la projection naturelle (voir par exemple [19, 1.5]). Cet opérateur se prolonge à $\mathcal{H}\left(G_{\infty}\right) \otimes_{\mathbb{Q}_{p} \otimes \mathbb{Z}_{p} \llbracket G_{\infty} \rrbracket} Z_{\infty}^{1}(K, V)$ (ce que nous noterons pour simplifier les notations $\mathcal{H}\left(G_{\infty}\right) \otimes Z_{\infty}^{1}(K, V)$. Une famille d'éléments $P_{n, k}$ de $H^{1}\left(K_{n}, V(k)\right)$ pour $a \leqslant k \leqslant b$ est dite tempérée d'ordre $\leqslant s$ s'il existe $z \in \mathcal{H}_{s}\left(G_{\infty}\right) \otimes Z_{\infty}^{1}(K, V)$ tel que $\pi_{n, k}(z)=P_{n, k}$ pour tout $a \leqslant k \leqslant b$ et pour tout $n \gg 0$.

5.2.3. ThÉORÈme. - Soit $h$ un entier $\geqslant 1$ tel que $\mathrm{Fil}^{-h} \mathbf{D}_{p}(V)=\mathbf{D}_{p}(V)$ et u tel que $u \geqslant 1-h$. Soit $g$ un élément de $\mathcal{D}_{\infty, g}(V)$ d'ordre $\leqslant u^{-}$et $G$ une solution complète de l'équation $(1-\Phi) G=g+\mathfrak{M}$. Les points de $H^{1}\left(K_{n}, V(k)\right)$

$$
P_{n, k}^{(h)}(G)=\exp _{V(k)}\left((-1)^{h+k-1}(h+k-1) ! \Xi_{n, k}(G)\right)
$$

pour $1-h \leqslant k \leqslant u$ et $n \gg 0$ forment une famille de points tempérée d'ordre $\leqslant(u+h)^{-}$ et définissent un élément de $\mathcal{H}\left(G_{\infty}\right) \otimes \widetilde{Z}_{\infty}^{1}(K, V)$ ne dépendant que de $g$ et que l'on note $\Omega_{V, h}^{u}(G)$ ou $\Omega_{V, h}^{u}(g)$. On a de plus

$$
\mathfrak{o}\left(\Omega_{V, h}^{u}(g)\right) \leqslant \mathfrak{o}_{\varphi}(g)+h .
$$

\subsubsection{REMARQUES}

1) Pour que $G$ et $g$ soient non nuls, il est nécessaire que $u \geqslant 1-h$.

2) On peut poser $\Gamma^{*}(1-h-k)=(-1)^{h+k-1}((h+k-1) !)^{-1}$, c'est le résidu de la fonction $\Gamma$ en $-k-h$ qui est ici négatif. On a donc

$$
P_{n, k}^{(h)}(G)=\frac{1}{\Gamma^{*}(1-h-k)} \exp _{V(k)}\left(\Xi_{n, k}(G)\right) .
$$

$3)$ « Pour $n$ assez grand signifie que $G$ est définie en $\zeta_{n}-1$.

4) Nous donnons dans l'appendice A.2 l'exemple des éléments cyclotomiques et de la fonction de Kubota-Leopoldt.

Démonstration. - Pour démontrer l'existence de $\Omega_{V, h}^{(u)}$, on procède comme dans [25]. Nous mettons ici l'accent sur ce qui est (un peu) nouveau. Les relations du type $\operatorname{Tr}_{n+1 / n}\left(P_{n+1, k}^{(h)}(G)\right)=P_{n, k}^{(h)}(G)$ sont impliquées par la propriété

$$
(1 \otimes \varphi)^{-1} \psi\left(D^{r}(G)\right)=p^{r} D^{r}(G)+(1 \otimes \varphi)^{-1} \psi\left(D^{r}(\mathfrak{M})\right) .
$$

Remarquons aussi qu'il suffit de regarder le cas où $K=\mathbb{Q}_{p}$ par induction. Notons Eul : $B_{\text {st }} \otimes \mathbf{D}_{p}(V) \rightarrow \operatorname{Fil}^{0}\left(B_{\text {st }} \otimes \mathbf{D}_{p}(V)\right)$ un scindage continu de l'application

$$
1-\Phi: \mathrm{Fil}^{0}\left(B_{\mathrm{st}} \otimes \mathbf{D}_{p}(V)\right) \longrightarrow B_{\mathrm{st}} \otimes \mathbf{D}_{p}(V) \text {. }
$$


L'image de l'application

$$
\begin{aligned}
& B_{\mathrm{st}} \otimes \mathbf{D}_{p}(V) / V \\
& \quad \longrightarrow B_{\mathrm{st}} \otimes \mathbf{D}_{p}(V) \oplus B_{\mathrm{st}} \otimes \mathbf{D}_{p}(V) \oplus B_{\mathrm{dR}} \otimes \mathbf{D}_{p}(V) / \mathrm{Fil}^{0}\left(B_{\mathrm{dR}} \otimes \mathbf{D}_{p}(V)\right)
\end{aligned}
$$

est formée des triplets $(A, B, C)$ tels que $N A=(1-p \Phi) B$. Soit $e_{B}$ un «scindage » continu de cette application sur son image. Si $(A, B, C) \in B_{\mathrm{st}} \otimes \mathbf{D}_{p}(V) \oplus B_{\mathrm{st}} \otimes$ $\mathbf{D}_{p}(V) \oplus B_{\mathrm{dR}} \otimes \mathbf{D}_{p}(V) / \operatorname{Fil}^{0}\left(B_{\mathrm{dR}} \otimes \mathbf{D}_{p}(V)\right)$ et si $C^{\prime}$ est un élément de $B_{\mathrm{st}} \otimes \mathbf{D}_{p}(V)$ tel que $\left.C^{\prime} \equiv C \bmod \mathrm{Fil}^{0}\left(B_{\mathrm{dR}} \otimes \mathbf{D}_{p}(V)\right)\right)$ et tel que $N C^{\prime}=A$ (cela existe et nous en trouverons!), on a

$$
e_{B}(A, B, C)=C^{\prime}-\operatorname{Eul}\left((1-\Phi) C^{\prime}-B\right) \bmod V
$$

et $N e_{B}(A, B, C)=A,(1-\Phi)\left(e_{B}(A, B, C)\right)=B$ et

$$
e_{B}(A, B, C) \equiv C \bmod \operatorname{Fil}^{0}\left(B_{\mathrm{dR}} \otimes \mathbf{D}_{p}(V)\right) .
$$

Si $b \in B_{\text {st }} \otimes \mathbf{D}_{p}(V)$, on a

$$
e_{B}(A+N b, B+(1-\Phi) b, C+b)=e_{B}(A, B, C)+b .
$$

On doit montrer que pour $j$ entier tel que $1-h \leqslant j \leqslant u$, la limite des

$$
Y_{n}^{(j)}=p^{\lfloor n(u+h-(j+h-1))\rfloor}(\tau-1) e_{B}\left(\sum_{k=1-h}^{j}(-1)^{k+h-1}\left(\begin{array}{l}
j+h-1 \\
k+h-1
\end{array}\right) \Xi_{n, k}^{(h)} t^{-k}\right)
$$

tend vers 0 lorsque $n \rightarrow \infty$ pour $\tau \in G_{\mathbb{Q}_{p}\left(\mu_{p} \infty\right)}$ avec

$$
\Xi_{n, k}^{(h)}=(-1)^{h+k-1}(h+k-1) ! \Xi_{n, k}(G) .
$$

Posons

$$
\mathcal{X}_{n}^{(j)}=p^{-n j}(j+h-1) !^{-1} \sum_{k=1-h}^{j}(-1)^{k+h-1}\left(\begin{array}{l}
j+h-1 \\
k+h-1
\end{array}\right) p^{n} \Xi_{n, k}^{(h)} t^{-k} .
$$

Ainsi, $Y_{n}^{(j)}=(j+h-1) ! p^{\lfloor n u\rfloor}(\tau-1) e_{B}\left(\mathcal{X}_{n}^{(j)}\right)$. On a avec $H=\left(\Lambda^{[1]}, \mathfrak{M}, G\right)$

$$
\begin{aligned}
\mathcal{X}_{n}^{(j)} & =\sum_{k=1-h}^{j} \frac{p^{n(k-j)}}{(j-k) !}(1 \otimes \varphi)^{-n} D^{-k}(H)\left(\zeta_{n}-1\right) t^{-k} \\
& =\sum_{k=0}^{h+j-1} \frac{p^{-n k}}{k !}(1 \otimes \varphi)^{-n} D^{-j+k}(H)\left(\zeta_{n}-1\right) t^{k-j} .
\end{aligned}
$$

Appliquons la formule de Taylor à $D^{-j}(G)$ :

$$
t^{-j} D^{-j}(G)\left(\beta_{n}-1\right)-t^{-j} \sum_{k=0}^{h+j-1} \frac{1}{p^{n k} k !} D^{k}\left(D^{-j}(G)\right)\left(\zeta_{n}-1\right) t^{k}
$$

appartient à $t^{-j} \mathrm{Fil}^{h+j} B_{\mathrm{dR}} \otimes \mathbf{D}_{p}(V) \subset \operatorname{Fil}^{0}\left(B_{\mathrm{dR}} \otimes \mathbf{D}_{p}(V)\right)$. On en déduit que la troisième composante $\mathcal{X}_{3}$ de $\mathcal{X}_{n}^{(j)}$ est congrue modulo $\operatorname{Fil}^{0}\left(B_{\mathrm{dR}} \otimes \mathbf{D}_{p}(V)\right)$ à 
$(1 \otimes \varphi)^{-n} D^{-j}(G)\left(\beta_{n}-1\right) t^{-j}$. Comme $e_{B}$ ne dépend de la troisième composante $\mathcal{X}_{3}$ que modulo $\mathrm{Fil}^{0}\left(B_{\mathrm{dR}} \otimes \mathbf{D}_{p}(V)\right)$, on peut donc la remplacer par

$$
(1 \otimes \varphi)^{-n} D^{-j}(G)\left(\beta_{n}-1\right) t^{-j}=p^{-n j} \Phi^{-n}\left(D^{-j}(G)([\varepsilon]-1) t^{-j}\right) .
$$

Les première et seconde composantes $\mathcal{X}_{1}$ et $\mathcal{X}_{2}$ de $\mathcal{X}_{n}^{(j)}$ valent avec $\mathcal{S}^{1}=\Lambda^{[1]}$ et $\mathcal{S}^{2}=\mathfrak{M}$

$$
\begin{aligned}
\sum_{k=0}^{h+j-1} \frac{p^{-n k}}{k !}(1 \otimes \varphi)^{-n} D^{-j+k}\left(\mathcal{S}^{i}\right)(0) t^{k-j} & =\sum_{k=0}^{h+j-1} \frac{p^{-n k}}{k !}(1 \otimes \varphi)^{-n} \mathcal{S}_{-j+k}^{i} t^{k-j} \\
& =(1 \otimes \varphi)^{-n} D^{-j}\left(\mathcal{S}^{i}\right)\left(\beta_{n}-1\right) t^{-j} \\
& =p^{-n j} \Phi^{-n}\left(D^{-j}\left(\mathcal{S}^{i}\right)([\varepsilon]-1) t^{-j}\right)
\end{aligned}
$$

Posons

$$
\left.b=p^{-n j} \sum_{r=1}^{n} \Phi^{-r}\left(D^{-j}(\mathfrak{M})\right)([\varepsilon]-1) t^{-j}\right)
$$

c'est un élément de $B_{\mathrm{st}}^{G_{\mathbb{Q}_{p}\left(\mu_{p} \infty\right)}} \otimes \mathbf{D}_{p}(V)$. On a

$$
\begin{aligned}
& p^{n j}\left(\mathcal{X}_{1}-N b\right)\left.=p^{n j} \mathcal{X}_{1}-\sum_{r=1}^{n} p^{-r} \Phi^{-r}\left(D^{-j}(N \mathfrak{M})([\varepsilon]-1)\right) t^{-j}\right) \\
&=p^{n j} \mathcal{X}_{1}-\sum_{r=1}^{n} p^{-r} \Phi^{-r}\left(D^{-j}((1-p \Phi) \Lambda)([\varepsilon]-1) t^{-j}\right) \\
&=p^{-n} \Phi^{-n}\left(D^{-j}(\Lambda)([\varepsilon]-1) t^{-j}\right) \\
& \quad-\sum_{r=1}^{n} p^{-r} \Phi^{-r}(1-p \Phi)\left(D^{-j}(\Lambda)([\varepsilon]-1) t^{-j}\right) \\
&= D^{-j}(\Lambda)([\varepsilon]-1) t^{-j}, \\
& p^{n j}\left(\mathcal{X}_{2}-(1-\Phi) b\right)= \Phi^{-n}\left(D^{-j}(\mathfrak{M})([\varepsilon]-1) t^{-j}\right) \\
&= \quad D^{-j}(\mathfrak{M})([\varepsilon]-1) t^{-j}, \\
& p^{n j}\left(\mathcal{X}_{3}-b\right) \equiv \Phi^{-n}\left(D^{-j}(G)([\varepsilon]-1) t^{-j}\right)-\sum_{r=1}^{n} \Phi^{-r}\left(D^{-j}(\mathfrak{M})([\varepsilon]-1) t^{-j}\right),
\end{aligned}
$$

ce qui peut aussi s'écrire

$$
p^{n j} \Phi^{-n}\left(D^{-j}(G)([\varepsilon]-1)\right) t^{-j}-\sum_{r=1}^{n} p^{r j} \Phi^{-r}\left(D^{-j}(\mathfrak{M})([\varepsilon]-1)\right) t^{-j}
$$

Bref, en réintroduisant la base canonique de $\mathbb{Q}_{p}[-k], \mathcal{X}_{3}-b$ est congru à la suite

$$
V_{n, j}=V_{n}\left(D^{-j}(G) \otimes e_{-j}\right) t^{-j}=V_{n, \mathbf{D}_{p}(V)[-j]}\left(D^{-j}(G) \otimes e_{-j}\right) t^{-j} .
$$


Elle converge dans $B_{\mathrm{st}} \otimes \mathbf{D}_{p}(V)$ dès que $\left\|p^{n j}(1 \otimes \varphi)^{-n} g\right\|^{(n)}$ tend vers 0 , i.e. dès que $g$ est d'ordre $\leqslant j^{-}$.

Les propriétés de $e_{B}$ impliquent que pour $\tau \in G_{\mathbb{Q}_{p}\left(\mu_{p} \infty\right)}$,

$$
\begin{aligned}
p^{n j} & (\tau-1) e_{B}\left(\mathcal{X}_{n}^{(j)}\right) \\
& =(\tau-1) e_{B}\left(D^{-j}(\Lambda)([\varepsilon]-1) t^{-j}, D^{-j}(\mathfrak{M})([\varepsilon]-1) t^{-j}, V_{n, j}\right)+(\tau-1) b \\
& =(\tau-1) e_{B}\left(D^{-j}(\Lambda)([\varepsilon]-1) t^{-j}, D^{-j}(\mathfrak{M})([\varepsilon]-1) t^{-j}, V_{n, j}\right) .
\end{aligned}
$$

Revenons à $Y_{n}^{(j)}=(j+h-1) ! p^{\lfloor n(u-j)\rfloor}(\tau-1) e_{B}\left(p^{n j} \mathcal{X}_{n}^{(j)}\right)$. Supposons d'abord que $j=u$. Alors, par continuité de $e_{B}, Y_{n}^{(j)}$ converge vers $(j+h-1) !(\tau-1) e_{B}\left(L_{1}, L_{2}, L_{3}\right)$ avec

$$
\begin{aligned}
& L_{1}=D^{-u}(\Lambda)([\varepsilon]-1) t^{-u}, \\
& L_{2}=D^{-u}(\mathfrak{M})([\varepsilon]-1) t^{-u}, \\
& L_{3}=\mathcal{L}_{\mathbf{D}_{p}(V)[-u]}\left(D^{-u}(G)\right) t^{-u} .
\end{aligned}
$$

Comme $L_{3} \in B_{\mathrm{st}}^{G_{\mathbb{Q}_{p}\left(\mu_{p} \infty\right)}} \otimes \mathbf{D}_{p}(V)$ et que $N L_{3}=L_{1}$ et $(1-\Phi)\left(L_{3}\right)=L_{2}$,

$$
e_{B}\left(L_{1}, L_{2}, L_{3}\right) \equiv L_{3}-\operatorname{Eul}\left((1-\Phi)\left(L_{3}\right)-L_{2}\right) \equiv L_{3} \bmod V
$$

et

$$
(\tau-1) e_{B}\left(L_{1}, L_{2}, L_{3}\right)=(\tau-1) L_{3}+(\tau-1) v
$$

pour $\tau \in G_{\mathbb{Q}_{p}\left(\mu_{p} \infty\right)}$ et $v \in V$ est un cobord, ce qui termine la démonstration dans le cas $j=u$.

Prenons maintenant $j<u$. La suite $C_{n}=\left\|p^{\lfloor n u\rfloor}(1 \otimes \varphi)^{-n} D^{-j}(g)\right\|^{(n)}$ tend vers 0 par hypothèse. On a

$$
\begin{aligned}
p^{n j} \Phi^{-n} & \left(D^{-j}(G)([\varepsilon]-1)\right)-\sum_{r=1}^{n} p^{n r} \Phi^{-r}\left(D^{-j}(\mathfrak{M})([\varepsilon]-1)\right) \\
& =p^{n j}(1 \otimes \varphi)^{-n} D^{-j}(G)\left(\beta_{n}-1\right)-\sum_{r=1}^{n} p^{r j}(1 \otimes \varphi)^{-r} D^{-j}(\mathfrak{M})\left(\beta_{r}-1\right) \\
& =P_{n}\left(\beta_{n}-1\right) .
\end{aligned}
$$

avec $P_{n}(x)=p^{n j}(1 \otimes \varphi)^{-n} D^{-j}(G)(x)-\sum_{r=1}^{n} p^{r j}(1 \otimes \varphi)^{-r} D^{-j}(\mathfrak{M})\left(\varphi^{n-r}(x)\right)$. Comme $D^{-j}(G)=p^{-j} \Phi\left(D^{-j}(G)\right)+D^{-j}(g)+D^{-j}(\mathfrak{M})$,

on trouve

$$
P_{n+1}=(\varphi \otimes 1) P_{n}+p^{(n+1) j}(1 \otimes \varphi)^{-(n+1)} D^{-j}(g)
$$

et donc

$$
\left\|p^{\lfloor(n+1)(u-j)\rfloor} P_{n+1}\right\|^{(n+1)} \leqslant \sup \left(C_{n+1}, p^{j-u}\left\|p^{\lfloor(n-1)(u-j)\rfloor} P_{n}\right\|^{(n)}\right)
$$

On en déduit que pour $j<u$, la suite $\left\|p^{\lfloor(n-1)(u-j)\rfloor} P_{n}\right\|^{(n)}$ tend vers 0 lorsque $n \rightarrow \infty$ pour $j<u$ et qu'il en est de même de la suite $Y_{n}^{(j)}$ (lemme 5.1.5). 
Ce qui démontre l'existence de $\Omega_{V, h}^{u}(G)$. L'inégalité

$$
\mathfrak{o}\left(\Omega_{V, h}^{u}(x)\right) \leqslant \mathfrak{o}_{\varphi}(g)+h
$$

est claire par la démonstration.

5.2.5. Nous n'avons interpolé qu'un nombre fini de tordus de $V$ ( $j$ est compris entre $1-h$ et $u$ ). Nous verrons un peu plus tard comment compléter le théorème et enlever l'exposant $u$ de $\Omega$. Dans le cas cristallin, on peut dès maintenant prendre $j \geqslant 1-h$. En effet, on remarque que si $y$ est un élément de $B_{\text {cris }} \otimes \mathbf{D}_{p}(V)$, on a $e_{B}(0,0, y)=y-\operatorname{Eul}((1-\varphi) y)$. Pour $g \in \mathcal{D}_{\infty, e}(\mathcal{D})$, comme $D^{-j}(G) \in \mathcal{H} \otimes \mathbf{D}_{p}(V)$, $(1 \otimes \varphi)^{-n} D^{-j}(G)\left(\beta_{n}-1\right) t^{-j}$ appartient à $B_{\text {cris }} \otimes \mathbf{D}_{p}(V)$, on a donc pour $\tau \in G_{K_{\infty}}$,

$$
\begin{aligned}
Y_{n}^{(j)}=p^{n u}(j+h-1) !(\tau-1)\left((1 \otimes \varphi)^{-n} D^{-j}(G)\left(\beta_{n}-1\right) t^{-j}\right) & \left.-\operatorname{Eul}\left((1 \otimes \varphi)^{-n} D^{-j}(g)\left(\beta_{n}-1\right) t^{-j}\right)\right) \\
& -p^{n u}(j+h-1) !(\tau-1) \operatorname{Eul}\left((1 \otimes \varphi)^{-n} D^{-j}(g)\left(\beta_{n}-1\right) t^{-j}\right) .
\end{aligned}
$$

Comme $p^{n u}(1 \otimes \varphi)^{-n} D^{-j}(g)\left(\beta_{n}-1\right) t^{-j}$ tend vers 0 , on en déduit que $Y_{n}^{(j)}$ tend vers 0 pour tout $j \geqslant 1-h$. Dans le cas de mauvaise réduction, cet argument ne s'applique pas car les $Y_{n}^{(j)}$ ne sont pas a priori dans $B_{\text {st }} \otimes \mathbf{D}_{p}(V)$.

5.2.6. Soit

$$
\ell_{h}=\frac{\log \gamma}{\log \chi(\gamma)}-h=\frac{\log \chi(\gamma)^{-h} \gamma}{\log \chi(\gamma)}
$$

Vérifions que

$$
\Omega_{V, h+1}^{u}=\ell_{h} \Omega_{V, h}^{u}
$$

Prenons $G$ tel que $(1-\Phi) G-\mathfrak{M}(G)$ soit d'ordre $u^{-}$. On a

$$
P_{n, k}^{(h+1)}(G)=(-h-k) P_{n, k}^{(h)}(G) .
$$

Or $\Omega_{V, h}^{u}(G)$ (resp. $\left.\Omega_{V, h+1}^{u}(G)\right)$ interpole les $P_{n, k}^{(h)}(G)$ (resp. $P_{n, k}^{(h+1)}(G)$ ) pour $-h \leqslant k \leqslant u$ (resp. $-h-1 \leqslant k \leqslant u$ ). On remarque d'autre part que comme Fil $^{-h} \mathbf{D}_{p}(V)=\mathbf{D}_{p}(V)$, les points $P_{n,-h}^{(h+1)}(G)$ sont en fait nuls car

$$
\mathrm{Fil}^{0} \mathbf{D}_{p}(V(-h))=\mathrm{Fil}^{0} \mathbf{D}_{p}(V)[h]=\mathrm{Fil}^{-h} \mathbf{D}_{p}(V)=\mathbf{D}_{p}(V) .
$$

Comme $\Omega_{V, h+1}^{u}(G)$ et $\ell_{h} \Omega_{V, h}^{u}(G)$ sont tous deux d'ordre $\leqslant(u+h+1)^{-}$, on en déduit facilement l'égalité.

On définit alors pour tout entier $h$ et pour $G$ tel que $(1-\Phi) G$ soit d'ordre $\leqslant u^{-}$,

$$
\Omega_{V, h}^{u}(G)=\left(\prod_{h \leqslant r<h^{\prime}} \ell_{j}\right)^{-1} \Omega_{V, h^{\prime}}^{u}(G)
$$

avec $h^{\prime}$ entier $>0$ et $\geqslant h$ tel que $\mathrm{Fil}^{-h^{\prime}} \mathbf{D}_{p}(V)=\mathbf{D}_{p}(V)$. 


\subsection{Loi de réciprocité}

5.3.1. Soit $W$ une représentation $p$-adique semi-stable de $K$. Si $L$ est une extension finie de $K$, soit $\lambda_{W, L}$ l'application duale de l'exponentielle $\exp _{W^{*}(1), L, g}$. Précisément, soit $\langle\cdot, \cdot\rangle_{V, L}$ le cup produit

$$
H^{1}(L, W) \times H^{1}\left(L, W^{*}(1)\right) \longrightarrow H^{1}\left(L, \mathbb{Q}_{p}(1)\right) \cong \mathbb{Q}_{p}
$$

et $[\cdot, \cdot]_{\mathbf{D}_{\mathrm{dR}}(V)}$ la dualité naturelle $L \otimes \mathbf{D}_{\mathrm{dR}}(W) \times L \otimes \mathbf{D}_{\mathrm{dR}}\left(W^{*}(1)\right) \rightarrow L \stackrel{\operatorname{Tr}_{L / \mathbb{Q}_{p}}}{\longrightarrow} \mathbb{Q}_{p}$. Notons aussi $[\cdot, \cdot]_{\mathbf{D}_{p}(W)}$ la dualité naturelle

$$
\mathbf{D}_{p}(W) \times \mathbf{D}_{p}\left(W^{*}(1)\right) \longrightarrow \mathbb{Q}_{p} .
$$

Posons pour $G$ une solution complète : $(1-\Phi) G=g+\mathfrak{M}(G)$

$$
\widetilde{\Xi}_{n, k}(G)=p^{n(k-1)}(1 \otimes \varphi)^{-n} D^{-k}(G)\left(\zeta_{n}-1\right)-\sum_{r=1}^{n} p^{n(r-1)}(1 \otimes \varphi)^{-r} D^{-k}(\mathfrak{M}(G))(0) .
$$

5.3.2. ThÉorème (Colmez). - Soit h un entier tel que $\mathrm{Fil}^{-h} \mathbf{D}_{p}(V)=\mathbf{D}_{p}(V)$. Soit $g \in \mathcal{D}_{\infty, g}(V)$ d'ordre $\leqslant u^{-}$et $G$ une solution complète de l'équation $(1-\Phi) G=g$ : $(1-\Phi) G=g+\mathfrak{M}$. Si $k=-h$, on suppose de plus que

$$
D^{-h}(\mathfrak{M}(G))(0) \in\left(1-p^{h} \varphi\right) \mathbf{D}_{p}(V) .
$$

On a

$$
\lambda_{V(k), n}\left(\pi_{n, k}\left(\Omega_{V, h}^{u}(g)\right)\right) \equiv \frac{1}{(-k-h) !} \widetilde{\Xi}_{n, k}(G) \bmod V(k)^{G_{K}}
$$

pour tout entier $n \gg 0$ et pour $k \leqslant-h$.

Remarquons pour augmenter l'analogie avec les formules du théorème 5.2.3 que $1 /(-k-h) !=\Gamma(1-h-k)^{-1}$.

Pour $k=-h$, la restriction n'est réelle que si $V(-h)^{G_{K}}$ est non nul, ce qui implique alors que $V(-h)$ admet $V(-h)^{G_{K}} \cong \mathbb{Q}_{p}^{s}$ comme facteur direct car les valeurs propres de $\varphi$ sur $\mathbf{D}_{p}(V)$ sont de valuation $\geqslant-h$ puisque Fil ${ }^{-h} \mathbf{D}_{p}(V)=\mathbf{D}_{p}(V)$ (faible admissibilité). Par exemple, si Fil ${ }^{-h+1} \mathbf{D}_{p}(V)=\mathbf{D}_{p}(V)$, cela ne peut se produire. De même, lorsque $k<-h$, on peut choisir la solution $G$ de manière à ce que $D^{-k}(\mathfrak{M})(0)=0$. On a alors plus simplement

$$
\lambda_{V(k), n}\left(\pi_{n, k}\left(\Omega_{V, h}^{u}(g)\right)\right) \equiv \frac{p^{n(k-1)}(1 \otimes \varphi)^{-n} D^{-k}(G)\left(\zeta_{n}-1\right)}{(-k-h) !} .
$$

Lorsque $g \in \mathcal{D}_{\infty, e}(V)$, c'est un théorème de Colmez [6], énoncé de manière un peu différente. Comme nous venons d'en faire la remarque, on peut remplacer $G$ par $G_{1}$ vérifiant $D^{-k}\left(\mathfrak{M}\left(G_{1}\right)\right)(0)=0$ pour $k \leqslant-h$. Un petit calcul facile montre que $\widetilde{\Xi}_{n, k}(G)=\widetilde{\Xi}_{n, k}\left(G_{1}\right)$. D'autre part, si $g \in \mathcal{D}_{\infty, g}(V)$, il existe un élément $U$ de $\mathbb{Z}_{p} \llbracket G_{\infty} \rrbracket$ dont l'image dans $\mathbb{Z}_{p} \llbracket \operatorname{Gal}\left(\mathbb{Q}_{p}\left(\mu_{p^{n}}\right) / \mathbb{Q}_{p}\right) \rrbracket$ est non diviseur de zéro et tel que $g_{1}=T w^{-k}(U) \cdot g$ appartient à $\mathcal{D}_{\infty, e}(V)$. Le théorème pour $g_{1}$ et $k$ implique alors la formule pour $\lambda_{V(k), n}\left(\pi_{n, k}\left(\Omega_{V, h}^{u}(g)\right)\right)$. 
Pour la démonstration dans le cas où $g \in \mathcal{D}_{\infty, e}(V)$, reprenons le texte $[\mathbf{2 5}]$. Écrivons toujours $(1-\Phi) G=g$ avec $g \in \mathcal{D}_{\infty, g}(V)$ d'ordre $\leqslant u^{-}$. Au cours de la construction de $\Omega_{V, h}^{u}(G)$, nous avons trouvé explicitement un cocycle $Z_{n, k, \tau}$ de $G_{K_{n}}$ à valeurs dans $V$ représentant $\pi_{n, k}\left(\Omega_{V, h}^{u}(G)\right)$ pour $n \geqslant 0$ et $k+h-1 \geqslant 0: Z_{n, k, \tau}=\left(\chi(\tau)^{k} \tau-1\right) e_{n, k}$ pour $\tau \in G_{K_{n}}$.

5.3.3. Lemme. - Avec les notations précédentes, la suite

$$
p^{m} \sum_{j=0}^{u+h-1}(-1)^{j}\left(\begin{array}{c}
u+h-1 \\
j
\end{array}\right) e_{m, j-h+1}
$$

converge dans $B_{\mathrm{st}}^{G_{K_{\infty}}} \otimes \mathbf{D}_{p}(V)$ et on a pour tout entier $n \geqslant 0$ et tout entier $k$ tel que $k+u \geqslant 0$

$$
\begin{array}{r}
\lambda_{k, n}\left(\lim _{m \rightarrow \infty} p^{m} \sum_{j=0}^{u+h-1}(-1)^{j}\left(\begin{array}{c}
u+h-1 \\
j
\end{array}\right) e_{m, j-h+1}\right) \\
=\frac{(u+h-1) !}{(k+u) !} p^{-n k}(1 \otimes \varphi)^{-n} D^{k}(G)\left(\zeta_{n}-1\right) .
\end{array}
$$

En effet, en reprenant les calculs de la démonstration du théorème 5.2 .3 , on obtient

$$
p^{m} \sum_{j=0}^{u+h-1}(-1)^{j}\left(\begin{array}{c}
u+h-1 \\
j
\end{array}\right) e_{m, j-h+1}=(u+h-1) ! V_{m, u}
$$

avec $V_{m, u}=\left(p^{m u} \Phi^{-m}\left(D^{-u} G([\varepsilon]-1)\right)\right) t^{-u}$. Lorsque $m \rightarrow \infty, V_{m, u}$ tend vers $\mathcal{L}_{\mathcal{D}[-u]}\left(D^{-u}(G) \otimes e_{-u}\right) t^{-u}$ (proposition 5.1.4) et on a

$$
\lambda_{k, n}\left(\mathcal{L}_{\mathcal{D}[-u]}\left(D^{-u}(G) \otimes e_{-u}\right) t^{-u}\right)=\lambda_{k+u, n}\left(\mathcal{L}_{\mathcal{D}[-u]}\left(D^{-u}(G) \otimes e_{-u}\right)\right.
$$

dont le calcul est fait dans la proposition 5.1.7.

La démonstration du théorème se termine comme dans [25, 4.3.3] (utiliser le lemme VI.3.2 de $[6])$.

5.3.4. On peut maintenant calculer les $\pi_{n, k}\left(\Omega_{V, h}^{u}(G)\right)$ pour $k \geqslant u$. Pour tout $k \leqslant u$, $\pi_{n, k}\left(\Omega_{V, h}^{u+1}(G)\right)=\pi_{n, k}\left(\Omega_{V, h}^{u}(G)\right)$. Comme $\Omega_{V, h}^{u}(G)$ et $\Omega_{V, h}^{u+1}(G)$ sont d'ordre respectivement $h+u$ et $h+u+1$, ils sont égaux et on a donc pour $k=u+1$

$$
\pi_{n, u+1}\left(\Omega_{V, h}^{u}(G)\right)=P_{n, u+1}^{(h)}(G) .
$$

On obtient le théorème suivant :

5.3.5. Théorème. - Soit $h$ un entier $\geqslant 1$ tel que $\operatorname{Fil}^{-h} \mathbf{D}_{p}(V)=\mathbf{D}_{p}(V)$. Soit $g \in \mathcal{D}_{\infty, g}(V)$ et $G$ une solution complète de l'équation $(1-\Phi) G=g$. Les points de $H^{1}\left(K_{n}, V(k)\right)$ pour $1-h \leqslant k$ et $n \gg 0$

$$
P_{n, k}^{(h)}(G)=\Gamma^{*}(-k-h)^{-1} \exp _{V(k)}\left(\Xi_{n, k}(G)\right)
$$


forment une famille tempérée de points et définissent un élément de $\mathcal{H}\left(G_{\infty}\right) \otimes$ $\widetilde{Z}_{\infty}^{1}(K, V)$ ne dépendant que de $g$ et noté $\Omega_{V, h}(g)$; son ordre est $\mathfrak{o}\left(\Omega_{V, h}(g)\right)=$ $\mathfrak{o}_{\varphi}(g)+h$. Pour tout entier $n \gg 0$

$$
\begin{aligned}
\lambda_{V(k), n}\left(\pi_{n, k}\left(\Omega_{V, h}(g)\right)\right) & \equiv \Gamma^{*}(1-h-k)^{-1} \widetilde{\Xi}_{n, k}(G) \text { pour } k \leqslant-h \\
\log _{V(k), n}\left(\pi_{n, k}\left(\Omega_{V, h}(g)\right)\right) & \equiv \Gamma^{*}(1-h-k)^{-1} \Xi_{n, k}(G) \text { pour } k>-h .
\end{aligned}
$$

Si Tw est l'opérateur de twist naturel $Z_{\infty}^{1}(K, V) \rightarrow Z_{\infty}^{1}(K, V(1))$, on a comme dans $[\mathbf{1 9}]$ et [25] la propriété

$$
\Omega_{V(1), h+1}(G)=\operatorname{Tw}\left(\Omega_{V, h}(D(G))\right) .
$$

Notons $\sigma_{-1}$ l'élément de $G_{\infty}$ agissant sur les racines de l'unité par $\zeta \mapsto \zeta^{-1}$. Soit $\iota$ l'involution de $\mathcal{H}\left(G_{\infty}\right)$ induite par $\tau \mapsto \tau^{-1}$. La notion de $\mathcal{H}\left(G_{\infty}\right)$-semi-linéarité sera relative à cet automorphisme de $\mathcal{H}\left(G_{\infty}\right)$ : linéarité à gauche, semi-linéarité à droite relativement à $\iota$. On note $\langle\cdot, \cdot\rangle_{V}$ l'accouplement semi-linéaire sur $Z_{\infty}^{1}(K, V) \times$ $Z_{\infty}^{1}\left(K, V^{*}(1)\right)$ déduit des accouplements de dualité sur $H^{1}\left(K_{n}, V\right) \times H^{1}\left(K_{n}, V^{*}(1)\right)$. On peut maintenant énoncer de manière différente le théorème de Colmez :

\subsubsection{Proposition. - La formule}

$$
\left[g_{1}, g_{2}\right]_{\mathbf{D}_{p}(V), \infty}=(-1)^{h}\left\langle\Omega_{V, h}\left(g_{1}\right), \sigma_{-1} \Omega_{V^{*}(1), 1-h}\left(g_{2}\right)\right\rangle_{V}
$$

définit un accouplement fonctoriel $\mathcal{H}\left(G_{\infty}\right)$-semi-linéaire

$$
\mathcal{D}_{\infty, f}\left(\mathbf{D}_{p}(V)\right) \times \mathcal{D}_{\infty, f}\left(\mathbf{D}_{p}\left(V^{*}(1)\right)\right) \longrightarrow \mathcal{H}\left(G_{\infty}\right)
$$

vérifiant

1) $\left[D\left(g_{1}\right), D^{-1}\left(g_{2}\right)\right]_{\mathbf{D}_{p}(V), \infty}=\mathrm{Tw}\left(\left[g_{1}, g_{2}\right]_{\mathbf{D}_{p}(V), \infty}\right)$

2) si $g_{1} \in \mathcal{D}_{\infty, f}\left(\mathbf{D}_{p}(V)^{N=0}\right)$,

$$
\left[g_{1}, g_{2}\right]_{\mathbf{D}_{p}(V), \infty} \cdot(1+x)=g_{1} * \pi\left(g_{2}\right)^{\iota}
$$

avec $\pi$ la projection dans $\mathcal{D}_{\infty, f}\left(\mathbf{D}_{p}\left(V^{*}(1)\right) / N\right)$ et $*$ induit par le produit de convolution « usuel»sur $\mathcal{H}^{\psi=0}$ et l'accouplement naturel $\mathbf{D}_{p}(V) \times \mathbf{D}_{p}\left(V^{*}(1)\right) \rightarrow \mathbb{Q}_{p}[-1] \cong \mathbb{Q}_{p}$.

Ainsi, si $h$ et $h^{*}$ sont des entiers tels que

$$
\mathrm{Fil}^{-h} \mathbf{D}_{p}(V)=\mathbf{D}_{p}(V), \mathrm{Fil}^{-h^{*}} \mathbf{D}_{p}\left(V^{*}(1)\right)=\mathbf{D}_{p}\left(V^{*}(1)\right),
$$

on a

$$
\left\langle\Omega_{V, h}\left(g_{1}\right), \sigma_{-1}\left(\prod_{-h<j<h^{*}} l_{-j}\right)^{-1} \Omega_{V^{*}(1), h^{*}}\left(g_{2}\right)\right\rangle_{V}=\left[g_{1}, g_{2}\right]_{\mathbf{D}_{p}(V), \infty} .
$$

La définition de cet accouplement est un peu tautologique. Cependant, la propriété (2) ne l'est pas et permet par exemple de montrer par dévissage que l'accouplement est unimodulaire, c'est-à-dire que l'image sur $\mathcal{D}_{\infty, f}\left(\mathbf{D}_{p}(V)\right) \times \mathcal{D}_{\infty, f}\left(\mathbf{D}_{p}\left(V^{*}(1)\right)\right)$ est exactement $\mathcal{H}\left(G_{\infty}\right)$, puisque c'est le cas du produit de convolution. On aimerait avoir une description directe de cet accouplement, par exemple une généralisation du produit de convolution à $\mathcal{B}[\log x]^{\psi=0}$. 
Si l'on prend $k$ tel que $-k \geqslant 1-h$ et $k<-h^{*}$, on peut appliquer les formules du théorème de définition.

Démonstration. - Soient $h$ et $h^{*}$ des entiers $\geqslant 1$ tels que $\operatorname{Fil}^{-h} \mathbf{D}_{p}(V)=\mathbf{D}_{p}(V)$ et $\mathrm{Fil}^{-h^{*}} \mathbf{D}_{p}(V)^{*}[-1]=\mathbf{D}_{p}(V)^{*}[-1]$. Posons

$$
\left[g_{1}, g_{2}\right]_{h, h^{*}}=(-1)^{h}\left\langle\Omega_{V, h}\left(g_{1}\right), \sigma_{-1} \Omega_{V^{*}(1), h^{*}}\left(g_{2}\right)\right\rangle_{V} .
$$

Cet accouplement est à valeurs dans $\mathcal{H}\left(G_{\infty}\right)$ et vérifie :

$$
\operatorname{Tw}^{k}\left(\left[g_{1}, g_{2}\right]_{h, h^{*}}\right)=\left[D^{-k}\left(g_{1}\right), D^{k}\left(g_{2}\right)\right]_{h, h^{*}} .
$$

L'image par la projection modulo $\operatorname{Gal}\left(K_{\infty} / K_{n}\right)$ de $\left[D^{-k}\left(g_{1}\right), D^{k}\left(g_{2}\right)\right]_{h, h^{*}}$ est nulle pour $k \geqslant-h+1$ et $-k>-h^{*}$ car les points $\pi_{n, k}\left(\Omega_{V, h}\left(g_{1}\right)\right)$ et $\pi_{n,-k}\left(\Omega_{V^{*}(1), h^{*}}\left(g_{2}^{\iota}\right)\right)$ sont alors respectivement dans $H_{f}^{1}\left(K_{n}, V\right)$ et $H_{f}^{1}\left(K_{n}, V^{*}(1)\right)$ et sont donc orthogonaux [2]. On en déduit que $\left[g_{1}, g_{2}\right]_{h, h^{*}}$ est divisible par $\ell_{-k}$ pour $-h<k<h^{*}$. On définit alors $\left[g_{1}, g_{2}\right]_{\mathbf{D}_{p}(V), \infty}$ comme $\prod_{-h<j<h^{*}} \ell_{-j}^{-1}\left[g_{1}, g_{2}\right]_{h, h^{*}}$, ce qui donne la formule de la proposition. Il est clair que l'accouplement est fonctoriel.

Prenons $g_{1} \in \mathcal{D}_{\infty, f}\left(\mathbf{D}_{p}(V)^{N=0}\right)$. Les formules trouvées au cours de la démonstration du théorème 4.2 .3 de $[\mathbf{2 5}]$ sont encore valables. On en déduit que $\left[g_{1}, g_{2}\right]_{\mathbf{D}_{p}(V), \infty}$ ne dépend que de la projection $\pi\left(g_{2}\right)$ de $g_{2}$ modulo $\mathcal{B}[\log x] \otimes N \mathbf{D}_{p}\left(V^{*}(1)\right)$. Comme $\pi\left(g_{2}\right) \in \mathcal{D}_{\infty, f}\left(\mathbf{D}_{p}\left(V^{*}(1)\right) / N\right)$, il est dans $\mathcal{H}^{\psi=0} \otimes \mathbf{D}_{p}\left(V^{*}(1)\right) / N$ et le produit de convolution $g_{1} * \pi\left(g_{2}\right)^{\iota}$ existe et est caractérisé par les formules citées.

Cet accouplement s'étend à $\mathcal{D}_{\infty, g}^{1}\left(\mathbf{D}_{p}(V)\right) \times \mathcal{D}_{\infty, g}^{1}\left(\mathbf{D}_{p}\left(V^{*}(1)\right)\right)$ à valeurs dans l'anneau total des fractions de $\mathcal{H}\left(G_{\infty}\right)$. On montre de même que

$$
\left[g_{1}, g_{2}\right]_{\mathbf{D}_{p}(V), \infty} \in \mathcal{H}\left(G_{\infty}\right)
$$

pour $g_{1} \in \mathcal{D}_{\infty, g}^{1}\left(\mathbf{D}_{p}(V)\right)$ et $g_{2} \in \mathcal{D}_{\infty, e}\left(\mathbf{D}_{p}\left(V^{*}(1)\right)\right)$.

5.3.7. Proposition. - Le déterminant de l'accouplement $[\cdot, \cdot]_{\mathbf{D}_{p}(V), \infty}$ sur

$$
\left(\otimes_{i \in\{1,2\}} \operatorname{det} \mathcal{D}_{\infty, g}^{i}\left(\mathbf{D}_{p}(V)\right)^{(-1)^{i+1}}\right) \otimes\left(\otimes_{i \in\{1,2\}} \operatorname{det} \mathcal{D}_{\infty, g}^{i}\left(\mathbf{D}_{p}(V)\right)^{(-1)^{i+1}}\right)
$$

est égal à $\mathcal{H}\left(G_{\infty}\right)$.

Rappelons que le déterminant d'un module de torsion s'envoie canoniquement dans $\operatorname{Frac}\left(\mathcal{H}\left(G_{\infty}\right)\right)$.

Démonstration. - La propriété à démontrer est multiplicative en les suites exactes de $(\varphi, N)$-modules grâce à la suite exacte (4.3.2). Par récurrence, il suffit de la montrer pour les $\varphi$-modules, ce qui se déduit du fait que dans ce cas

$$
\otimes_{i \in\{1,2\}}\left(\operatorname{det} \mathcal{D}_{\infty, g}^{i}(\mathcal{D})\right)^{(-1)^{i+1}} \cong \operatorname{det} \mathcal{D}_{\infty, f}(\mathcal{D})
$$

et que l'image du produit de convolution sur $\mathcal{H}\left(G_{\infty}\right)$ est exactement $\mathcal{H}\left(G_{\infty}\right)$. 


\subsection{Déterminant de $\Omega_{V, h}$}

Nous reprenons les notations des articles loc. cités. On note $\delta_{h}\left(\Omega_{V}\right)$ le sous- $\mathcal{H}\left(G_{\infty}\right)$ module de $\operatorname{Frac}\left(\mathcal{H}\left(G_{\infty}\right)\right)$ image du déterminant de $\Omega_{V, h}$ sur

$$
\begin{aligned}
\operatorname{det} \mathcal{D}_{\infty, g}^{1}(V) & \otimes\left(\operatorname{det} \mathcal{D}_{\infty, g}^{2}(V)\right)^{-1} \otimes\left(\operatorname{det} Z_{\infty}^{1}(K, V)\right)^{-1} \otimes \operatorname{det} Z_{\infty}^{2}(K, V) \\
& =\otimes_{i \in\{1,2\}}\left(\operatorname{det} \mathcal{D}_{\infty, g}^{i}(V)\right)^{(-1)^{i}} \otimes \otimes_{i \in\{1,2\}}\left(\operatorname{det} Z_{\infty}^{1}(K, V)\right)^{(-1)^{i+1}}
\end{aligned}
$$

et

$$
\delta_{\mathrm{st}}(V)=\prod_{j>-h} \ell_{-j}^{-\operatorname{dim}_{\mathbb{Q}_{p}} \operatorname{Fil}^{j} \mathbf{D}_{p}(V)} \delta_{h}\left(\Omega_{V}\right)
$$

qui est indépendant de $h$ à condition que $\mathrm{Fil}^{-h} \mathbf{D}_{p}(V)=\mathbf{D}_{p}(V)$.

Les suites exactes de cohomologie et la suite exacte (4.3.2) impliquent que $\delta_{\text {st }}$ est multiplicatif sur les suites exactes : si $0 \rightarrow V_{1} \rightarrow V_{1} \rightarrow V_{1} \rightarrow 0$ est une suite exacte de représentations $p$-adiques semi-stables, on a un isomorphisme naturel

$$
\delta_{\mathrm{st}}\left(V_{2}\right) \cong \delta_{\mathrm{st}}\left(V_{1}\right) \otimes \delta_{\mathrm{st}}\left(V_{3}\right) .
$$

De plus, si $V$ est cristalline, $\operatorname{det} \mathcal{D}_{\infty, g}^{1}(V) \otimes\left(\operatorname{det} \mathcal{D}_{\infty, g}^{2}(V)\right)^{-1}$ est naturellement isomorphe à $\operatorname{det} \mathcal{D}_{\infty, f}^{1}(V)$ car

$$
\begin{aligned}
& \mathcal{D}_{\infty, g}^{2}(V)=\mathcal{U}\left(\mathbf{D}_{p}(V)\right) /(1-p \Phi) \mathcal{U}\left(\mathbf{D}_{p}(V)\right. \\
& \mathcal{D}_{\infty, g}^{1}(V) / \mathcal{D}_{\infty, f}(V)=\mathcal{U}\left(\mathbf{D}_{p}(V)\right)^{\Phi=p^{-1}}
\end{aligned}
$$

En général,

$$
\begin{aligned}
\operatorname{det} \mathcal{D}_{\infty, g}^{1}\left(V \otimes\left(\operatorname{det} \mathcal{D}_{\infty, g}^{2}(V)\right)^{-1}=\operatorname{det} \mathcal{D}_{\infty, f}^{1}(V) \otimes\right. \\
\left(\operatorname{det} \mathcal{U}\left(\mathbf{D}_{p}(V)\right) /(1-p \Phi, N)\right)^{-1} \otimes\left(\operatorname{det} \mathcal{U}\left(\mathbf{D}_{p}(V)\right) / N \mathcal{U}\left(\mathbf{D}_{p}(V)\right)^{\Phi=p^{-1}}\right) .
\end{aligned}
$$

5.4.1. Proposition. - Le $\mathcal{H}\left(G_{\infty}\right)$-module $\delta_{\mathrm{st}}(V)$ est contenu dans $\mathcal{H}\left(G_{\infty}\right)$.

La démonstration consiste à reprendre [19]. Donnons-en les étapes. Posons $\mathcal{D}=\mathbf{D}_{p}(V)$. On commence par étudier $\mathcal{D}_{\infty, e}(\mathcal{D})_{G_{n}}$ où $G_{n}=\operatorname{Gal}\left(K_{\infty} / K_{n}\right)$. Considérons l'application linéaire de $G_{\infty}$-modules

$$
\xi_{n}: \mathcal{D}_{\infty, e}(\mathcal{D}) \longrightarrow\left(K_{n} \otimes \mathcal{D}\right) / \mathcal{D}^{N=0, \varphi=1},
$$

définie de la manière suivante : si $g \in \mathcal{D}_{\infty, e}(\mathcal{D})$, écrivons $g=(1-\Phi)(G)$ avec $N G=0$. L'équation fonctionnelle $\psi(G)=(1 \otimes \varphi) G$ implique que

$$
\left(p^{m-n} \operatorname{Tr}_{K_{m} / K_{n}}\left((1 \otimes \varphi)^{-m} G\left(\zeta_{m}-1\right)\right)\right.
$$

ne dépend pas de $m$ assez grand pour que $G\left(\zeta_{m}-1\right)$ soit définie. D'où l'application notée $\xi_{n}$

$$
\xi_{n}(\mathcal{D}): \mathcal{D}_{\infty, f}(\mathcal{D})_{G_{n}} \rightarrow K_{n} \otimes \mathcal{D} / \mathcal{D}^{N=0, \varphi=1} \stackrel{\text { déf }}{=} \mathcal{R}_{n}(\mathcal{D}) .
$$

On pose $h\left(\xi_{n}(\mathcal{D})\right)=\operatorname{dim} \operatorname{coker} \xi_{n}(\mathcal{D})-\operatorname{dim} \operatorname{ker} \xi_{n}(\mathcal{D})$.

5.4.2. Lemme. $-h\left(\xi_{n}(\mathcal{D})\right)=-\operatorname{dim} \mathcal{D}^{\varphi=1, N=0}, \operatorname{dim} \mathcal{D}_{\infty, e}(\mathcal{D})_{G_{n}}=\left[K_{n}: \mathbb{Q}_{p}\right] \operatorname{dim} \mathcal{D}$. 
Démonstration. - Soit $(\nu): 0 \rightarrow \mathcal{D}_{1} \rightarrow \mathcal{D}_{2} \rightarrow \mathcal{D}_{3} \rightarrow 0$ une suite exacte de $(\varphi, N)$ modules. Si $H$ est une fonction de $\mathcal{D}$ dans $\mathbb{R}$, on pose

$$
H(\nu)=\sum_{i=1}^{3}(-1)^{i} H\left(\mathcal{D}_{i}\right) \text {. }
$$

Il est clair que

$$
\operatorname{dim} \mathcal{R}_{n}(\nu)+\operatorname{dim} \nu^{\varphi=1, N=0}=0 .
$$

D'autre part,

$$
\begin{aligned}
\operatorname{dim}_{\mathbb{Q}_{p}} \mathcal{D}_{\infty, e}(\nu)_{G_{n}} & =\operatorname{dim}_{\mathbb{Q}_{p}} \mathcal{D}_{\infty, g}(\nu)_{G_{n}}+\operatorname{dim}_{\mathbb{Q}_{p}} \mathcal{C}_{\infty}(\nu)_{G_{n}}-\operatorname{dim}_{\mathbb{Q}_{p}} \mathcal{C}_{\infty}(\nu)^{G_{n}} \\
& =\operatorname{dim}_{\mathbb{Q}_{p}} \mathcal{D}_{\infty, g}(\nu)_{G_{n}}=0 .
\end{aligned}
$$

En effet on a une suite exacte

$$
0 \longrightarrow \mathcal{D}_{\infty, g}\left(\mathcal{D}_{1}\right) \longrightarrow \mathcal{D}_{\infty, g}(\mathcal{D}) \longrightarrow \mathcal{D}_{\infty, g}\left(\mathcal{D}_{2}\right) \longrightarrow U \longrightarrow 0
$$

où $U$ est un $\mathbb{Q}_{p}$-espace vectoriel de dimension finie. Comme $\operatorname{dim}_{\mathbb{Q}_{p}} U^{G_{n}}=\operatorname{dim}_{\mathbb{Q}_{p}} U_{G_{n}}$ et que $\mathcal{D}_{\infty, g}(\mathcal{D})^{G_{n}}=0$ pour tout $(\varphi, N)$-module, $\operatorname{dim}_{\mathbb{Q}_{p}} \mathcal{D}_{\infty, g}(\nu)_{G_{n}}=0$. En utilisant les suites exactes tautologiques

$$
0 \longrightarrow \operatorname{ker} \xi_{n}(\mathcal{D}) \longrightarrow \mathcal{D}_{\infty, e}(\mathcal{D})_{G_{n}} \longrightarrow \mathcal{R}_{n}(\mathcal{D}) \longrightarrow \operatorname{coker} \xi_{n}(\mathcal{D}) \longrightarrow 0
$$

on obtient que

$$
h\left(\xi_{n}(\nu)\right)+\operatorname{dim} \nu^{\varphi=1, N=0}=0 .
$$

Lorsque $\mathcal{D}$ est un $\varphi$-module, $\operatorname{dim}_{\mathbb{Q}_{p}} \mathcal{D}_{\infty, e}(\mathcal{D})_{G_{n}}=\left[K_{n}: \mathbb{Q}_{p}\right] \operatorname{dim} \mathcal{D}$ et grâce à $[\mathbf{1 9}$, $3.4 .4]$

$$
\begin{aligned}
\operatorname{dim} \mathcal{D}^{\varphi=1, N=0}+h\left(\xi_{n}(\mathcal{D})\right)= & \operatorname{dim} \mathcal{D}^{\varphi=1, N=0}-\operatorname{dim} \mathcal{D}^{N=0} /(1-\varphi) \\
& \quad-\operatorname{dim}(\mathcal{D} / N \mathcal{D})^{\varphi=p^{-1}}+\operatorname{dim} \mathcal{D}^{N=0} /(1-p \varphi) \\
= & 0 .
\end{aligned}
$$

Donc pour tout $(\varphi, N)$-module,

$$
\begin{aligned}
h\left(\xi_{n}(\mathcal{D})\right) & =-\operatorname{dim} \mathcal{D}^{\varphi=1, N=0}, \\
\operatorname{dim}_{\mathbb{Q}_{p}} \mathcal{D}_{\infty, e}(\mathcal{D})_{G_{n}} & =\left[K_{n}: \mathbb{Q}_{p}\right] \operatorname{dim} \mathcal{D} .
\end{aligned}
$$

Calculons la dimension sur $\mathbb{Q}_{p}$ du noyau de

$$
\Omega_{V, h, n}: \mathcal{D}_{\infty, e}(V)_{G_{n}} \longrightarrow Z_{\infty}^{1}(K, V)_{G_{n}} .
$$

5.4.3. Lemme. - $\operatorname{dim} \operatorname{ker} \Omega_{V, h, n} \geqslant\left[K_{n}: \mathbb{Q}_{p}\right] \operatorname{dim} \operatorname{Fil}^{0} \mathcal{D}-\operatorname{dim} V^{G_{K}}+\operatorname{dim} \mathcal{D}^{\varphi=1, N=0}$. Démonstration. - L'application $\mathcal{D}_{\infty, e}(V) \rightarrow H_{e}^{1}\left(K_{n}, V\right)$ se factorise en une application

$$
\Omega_{V, h, n}^{\prime}: \mathcal{D}_{\infty, e}(V)_{G_{n}} \longrightarrow H_{e}^{1}\left(K_{n}, V\right)
$$


Comme $Z_{\infty}^{1}(K, V)_{G_{n}}$ s'injecte dans $H^{1}\left(K_{n}, V\right)$, le noyau de $\Omega_{V, h, n}^{\prime}$ est égal au noyau de $\Omega_{V, h, n}$. On a alors le diagramme commutatif suivant

$$
\begin{aligned}
& 0 \\
& \downarrow \\
& \operatorname{ker} \xi_{n} \quad 0 \\
& 0 \rightarrow \operatorname{ker} \Omega_{V, n, h}^{\prime} \rightarrow \mathcal{D}_{\infty, e}(V)_{G_{n}} \rightarrow H_{e}^{1}\left(K_{n}, V\right) \\
& V^{G_{K}} \rightarrow \mathcal{S}_{n}(\mathcal{D}) \rightarrow \stackrel{\downarrow}{\downarrow} \rightarrow \mathcal{R}_{n}(\mathcal{D}) \rightarrow H_{e}^{1}\left(K_{n}, V\right) \\
& \begin{array}{cc}
\downarrow & \downarrow \\
\operatorname{coker} \xi_{n}(\mathcal{D}) & 0
\end{array} \\
& \downarrow
\end{aligned}
$$

avec $\mathcal{S}_{n}(\mathcal{D})=K_{n} \otimes \mathrm{Fil}^{0} \mathcal{D} / \mathrm{Fil}^{0} \mathcal{D}^{N=0, \varphi=1}$ et $\mathcal{R}_{n}(\mathcal{D})=K_{n} \otimes \mathcal{D} / \mathcal{D}^{N=0, \varphi=1}$ (rappelons que $\mathrm{Fil}^{0} \mathcal{D}^{N=0, \varphi=1}=V^{G_{K}}=V^{G_{K_{n}}}$ car $V$ est semi-stable). On en déduit une suite exacte

$$
0 \longrightarrow \operatorname{ker} \xi_{n}(\mathcal{D}) \longrightarrow \operatorname{ker} \Omega_{V, h, n}^{\prime} \longrightarrow \mathcal{S}_{n}(\mathcal{D}) / V^{G_{K_{n}}} \longrightarrow \operatorname{coker} \xi_{n}(\mathcal{D})
$$

et l'inégalité

$$
\operatorname{dim} \operatorname{ker} \Omega_{V, h, n}^{\prime} \geqslant\left[K_{n}: \mathbb{Q}_{p}\right] \operatorname{dim} \operatorname{Fil}^{0} \mathcal{D}-\operatorname{dim} V^{G_{K}}-\operatorname{dim} h\left(\xi_{n}(\mathcal{D})\right) .
$$

Finissons la démonstration de la proposition 5.4.1. En procédant comme dans $[\mathbf{1 9}$, $3.4]$, on montre que le déterminant de $\Omega_{V, h}$ sur

$$
\operatorname{det} \mathcal{D}_{\infty, e}(\mathcal{D}) \otimes \operatorname{det} \mathcal{U}(\mathcal{D})^{N=0, \Phi=1} \otimes\left(\operatorname{det} V^{G_{K_{\infty}}}\right)^{-1}
$$

est divisible par $\prod_{j>-h} \ell_{-j}^{\operatorname{dim}_{\mathbb{Q}_{p}} \mathrm{Fil}^{j} \mathcal{D}}$ dans $\mathcal{H}\left(G_{\infty}\right)$. Rappelons que

$$
\operatorname{det} Z_{\infty}^{2}(K, V)=\left(\operatorname{det} V^{*}(1)^{G_{K_{\infty}}}\right)^{\iota} .
$$

Donc $\left(\operatorname{det} V^{G_{K_{\infty}}}\right)^{-1} \cdot \delta_{\mathrm{st}}(V)$ est contenu dans

$$
\begin{aligned}
&\left(\operatorname{det} \mathcal{U}(\mathcal{D})^{N=0, \Phi=1}\right)^{-1} \cdot \operatorname{det} \mathcal{D}_{\infty, g}(\mathcal{D}) / \mathcal{D}_{\infty, e}(\mathcal{D}) \cdot\left(\operatorname{det} \mathcal{D}_{\infty, g}^{2}(\mathcal{D})\right)^{-1} \\
&=\left(\operatorname{det} \mathcal{U}(\mathcal{D})^{N=0, \Phi=1}\right)^{-1} \cdot \operatorname{det}(\mathcal{U}(\mathcal{D}) / N \mathcal{U}(\mathcal{D}))^{\Phi=p^{-1}} \\
& \quad \cdot\left(\operatorname{det} \mathcal{U}\left(\mathcal{D}^{N=0}\right) /(1-\Phi)\right) \cdot(\operatorname{det} \mathcal{U}(\mathcal{D}) /(1-p \Phi, N) \mathcal{U}(\mathcal{D}))^{-1} \\
&=\mathcal{H}\left(G_{\infty}\right)
\end{aligned}
$$

d'où la proposition 5.4.1 lorsque $V^{*}(1)^{G_{K_{\infty}}}=0$. Dans le cas contraire, on peut soit raffiner la suite exacte comme dans $[\mathbf{1 9}, 3.4 .5]$, soit se ramener au cas où $V^{*}(1)^{G_{K_{\infty}}}=0$ par dévissage (la proposition est «multiplicative» en les suites exactes) en invoquant le résultat de [19] puisque $V^{G_{K_{\infty}}}$ est cristalline. 
Montrons maintenant que $\delta_{\text {st }}(V)=\mathcal{H}\left(G_{\infty}\right)$. Le $\mathbb{Q}_{p} \otimes \mathbb{Z}_{p} \llbracket G_{\infty} \rrbracket$-déterminant de l'accouplement de dualité sur

$$
\left(\otimes_{i \in\{1,2\}}\left(\operatorname{det} Z_{\infty}^{1}(K, V)\right)^{(-1)^{i+1}}\right) \otimes\left(\otimes_{i \in\{1,2\}}\left(\operatorname{det} Z_{\infty}^{1}\left(K, V^{*}(1)\right)\right)^{(-1)^{i+1}}\right)^{\iota}
$$

est $\mathbb{Q}_{p} \otimes \mathbb{Z}_{p} \llbracket G_{\infty} \rrbracket$ (le module de torsion de $Z_{\infty}^{1}(K, V)$ a même série caractéristique que $\left.Z_{\infty}^{2}\left(K, V^{*}(1)\right)^{\iota}\right)$. En utilisant 5.3.7, on en déduit que

$$
\delta_{h}\left(\Omega_{V}\right) \delta_{1-h}\left(\Omega_{V *(1)}\right)^{\iota}=\mathcal{H}\left(G_{\infty}\right)
$$

et

$$
\delta_{\mathrm{st}}(V) \delta_{\mathrm{st}}\left(V^{*}(1)\right)^{\iota}=\mathcal{H}\left(G_{\infty}\right) .
$$

Comme on a les inclusions $\delta_{\text {st }}(V) \subset \mathcal{H}\left(G_{\infty}\right), \delta_{\text {st }}\left(V^{*}(1)\right) \subset \mathcal{H}\left(G_{\infty}\right)$, on obtient le théorème suivant :

5.4.4. ThÉORÈme. - Soit $V$ une représentation p-adique semi-stable de $G_{K}$. Alors, $\delta_{\mathrm{st}}(V)=\mathcal{H}\left(G_{\infty}\right)$.

Lorsque $V$ est cristalline, $\delta_{\text {st }}(V)$ provient naturellement d'un sous- $\mathbb{Q}_{p} \otimes \mathbb{Z}_{p} \llbracket G_{\infty} \rrbracket$ module $\delta_{\text {cris }}(V)$ de $\mathcal{H}\left(G_{\infty}\right)$ et on a $\delta_{\text {cris }}(V)=\mathbb{Q}_{p} \otimes \mathbb{Z}_{p} \llbracket G_{\infty} \rrbracket$.

Soient $h$ et $h^{*}$ des entiers $\geqslant 1$ tels que

$$
\mathrm{Fil}^{-h} \mathbf{D}_{p}(V)=\mathbf{D}_{p}(V), \quad \mathrm{Fil}^{-h^{*}} \mathbf{D}_{p}\left(V^{*}(1)\right)=\mathbf{D}_{p}\left(V^{*}(1)\right) .
$$

5.4.5. Proposition. - Si $x \in Z_{\infty}^{1}(K, V), \prod_{-h<j<h^{*}} \ell_{-j} x$ appartient à l'image de $\mathcal{D}_{\infty, f}(V) \operatorname{par} \Omega_{V, h}$.

Démonstration. - La démonstration est identique à celle de [25]. On a pour des entiers positifs $\alpha_{j}$

$$
\prod_{-h<j<h^{*}} \ell_{-j}^{\alpha_{j}} x=\lambda^{-1} \Omega_{V, h}(g)
$$

avec $\lambda \in \mathbb{Z}_{p} \llbracket G_{\infty} \rrbracket$ et $g \in \mathcal{D}_{\infty, f}\left(\mathbf{D}_{p}(V)\right)$. D'où, pour $g^{*} \in \mathcal{D}_{\infty, f}\left(\mathbf{D}_{p}\left(V^{*}(1)\right)\right)$,

$$
\begin{aligned}
\lambda\left\langle x, \Omega_{V^{*}(1), h^{*}}\left(g^{*}\right)\right\rangle & =\left\langle\left(\prod_{-h<j<h^{*}} \ell_{-j}\right)^{-\alpha_{j}} \Omega_{V, h}(g), \Omega_{V^{*}(1), h^{*}}\left(g^{*}\right)\right\rangle \\
& =\left(\prod_{-h<j<h^{*}} \ell_{-j}^{-\alpha_{j}+1}\right)\left\langle\Omega_{V, h}(g), \Omega_{V^{*}(1), 1-h}\left(g^{*}\right)\right\rangle \\
& =\left(\prod_{-h<j<h^{*}} \ell_{-j}^{-\alpha_{j}+1}\right)\left[g, g^{*}\right]_{\mathbf{D}_{p}(V), \infty}
\end{aligned}
$$

On en déduit que pour tout $g^{*} \in \mathcal{D}_{\infty, f}\left(\mathbf{D}_{p}\left(V^{*}(1)\right)\right)$,

$$
\left[g, g^{*}\right]_{\mathbf{D}_{p}(V), \infty} \in \lambda \prod_{-h<j<h^{*}} \ell_{-j}^{-\alpha_{j}+1} \mathcal{H}\left(G_{\infty}\right) .
$$


L'accouplement $[\cdot, \cdot]_{\mathbf{D}_{p}(V), \infty}$ est unimodulaire sur $\mathcal{D}_{\infty, f}(V) \times \mathcal{D}_{\infty, f}\left(V^{*}(1)\right)$. Donc $g \in \lambda \prod_{-h<j<h^{*}} \ell_{-j}^{-\alpha_{j}+1} \mathcal{D}_{\infty, f}(V)$. D'où l'existence de $g_{1} \in \mathcal{D}_{\infty, f}(V)$ tel que

$$
\prod_{-h<j<h^{*}} \ell_{-j} x=\Omega_{V, h}\left(g_{1}\right) .
$$




\section{CHAPITRE 6}

\section{NORMES UNIVERSELLES}

Nous avons maintenant tout ce qu'il nous faut pour démontrer le théorème suivant :

6.0.1. ThÉonÈmE. - Soient $V$ une représentation p-adique semi-stable d'une extension finie non ramifiée $K$ de $\mathbb{Q}_{p}$ et $T$ un réseau de $V$ stable par $G_{K}$. Soit $\mathrm{Fil}^{1} V$ la plus grande sous-représentation de $V$ dont les poids de Hodge-Tate sont strictement positifs et $\mathrm{Fil}^{1} T=\mathrm{Fil}^{1} V \cap T$. Alors, la limite projective des $H_{g}^{1}\left(K\left(\mu_{p^{n}}\right), T\right)$ relativement aux applications de corestriction est de rang sur $\mathbb{Z}_{p} \llbracket G_{\infty} \rrbracket$ égal à $\left[K: \mathbb{Q}_{p}\right] \operatorname{dim}$ Fil $^{1} V$ et égal à torsion près à la limite projective des $H^{1}\left(K\left(\mu_{p^{n}}\right), \mathrm{Fil}^{1} T\right)$.

Par exemple, si $V$ est une représentation semi-stable irréductible sur $\mathbb{Q}_{p}$, le $\mathbb{Z}_{p} \llbracket G_{\infty} \rrbracket$ module $Z_{\infty, g}^{1}\left(\mathbb{Q}_{p}, T\right)$ est nul ou égal à $Z_{\infty}^{1}\left(\mathbb{Q}_{p}, T\right)$.

La démonstration est identique à la démonstration de [26]. Nous indiquons dans ce qui suit quelques modifications à faire.

\subsection{Ingrédients}

Commençons par un lemme de divisibilité.

6.1.1. Lemme. - Soit $G \in(\mathcal{B}[\log x] \otimes \mathcal{D})^{N=0}$ tel que $G\left(\zeta_{n}-1\right)=0$ pour tout entier $n \gg 0$. Alors, il existe $G_{1} \in(\mathcal{B}[\log x] \otimes \mathcal{D})^{N=0}$ tel que $G=\log (1+x) G_{1}$. De plus, $\mathfrak{o}(G)=\mathfrak{o}\left(G_{1}\right)-1$.

Démonstration. - Pour $G \in \mathcal{H}^{[\rho, 1[}$, le lemme se déduit de la continuité de la division euclidienne comme dans le cas des éléments de $\mathcal{H}$ (remarquons que $\operatorname{Frac}\left(\mathbb{Z}_{p} \llbracket x \rrbracket\right)$ s'injecte dans $\mathcal{B})$. Passons à $G \in(\mathcal{B}[\log x] \otimes \mathcal{D})^{N=0}$. On a donc

$$
G=\sum_{k} \frac{(-1)^{k}}{k !} N^{k} G_{0} \log ^{k} x
$$

avec $G_{0} \in \mathcal{B} \otimes \mathcal{D}$. Comme $G\left(\zeta_{n}-1\right)=0$ pour tout entier $n$, il en est de même des $(1 \otimes N)^{j}(G)\left(\zeta_{n}-1\right)$. Raisonnons par récurrence sur le plus grand entier $r$ tel que 
$N^{r} G_{0} \neq 0$. On a alors $(1 \otimes N)^{r} G=(1 \otimes N)^{r} G_{0}=N^{r} G_{0}$. Donc

$$
(1 \otimes N)^{r} G_{0}=\log (1+x) H_{1}
$$

avec $H_{1}=N^{r} K_{1} \in \mathcal{B} \otimes \mathcal{D}$. Soit $G_{1}=G_{0}-(-1)^{r} \log (1+x) K_{1}$. Alors

$$
G=\sum_{k}\left((-1)^{k} / k !\right) N^{k} G_{1} \log ^{k} x-(-1)^{r} \log (1+x) H_{1}
$$

et $N^{r} G_{1}=0$. Par récurrence, on en déduit le lemme.

6.1.2. Le lemme $[\mathbf{2 6}, 2.2]$ sur les filtrations devient:

LEMme. - Soit $W$ un K-espace vectoriel de dimension finie muni d'un opérateur nilpotent $N$. Soient $g_{1}, \ldots, g_{d}$ des éléments de $\left(\mathcal{B}[\log x] \otimes_{K} W\right)^{N=0}$. On suppose que pour tout entier $n$, il existe une filtration décroissante exhaustive et séparée $\mathrm{Fil}^{i} W_{n}$ de $W_{n}^{k}=K_{n} \otimes_{K} W$ avec Fil $^{1} W_{n}=0$ telle que

1) les entiers $h_{j}=\operatorname{dim}_{K_{n}} \mathrm{Fil}^{j} W_{n}-\operatorname{dim}_{K_{n}} \mathrm{Fil}^{j+1} W_{n}$ ne dépendent pas de $n$;

2) pour tout entier $j \leqslant 0$ et toute racine de l'unité $\zeta_{n}$ d'ordre $p^{n}$,

$$
D^{-j}\left(g_{i}\right)\left(\zeta_{n}-1\right) \in \mathrm{Fil}^{j} W_{n} .
$$

Alors $\operatorname{det}\left(g_{1}, \ldots, g_{d}\right)$ (calculé dans une base de $W$ ) est divisible par $\log ^{-t_{H}}(1+x)$ où $t_{H}=\sum_{j} j h_{j}$ est le degré de la filtration.

6.1.3. Le lemme suivant est très important car il implique que tous les sous- $\varphi$-modules de $\mathbf{D}_{p}(V)$ qui interviennent dans la démonstration sont nécessairement stables par $N$ et donc des $(\varphi, N)$-modules.

LEMmE. - Soit $\mathcal{D}$ un $(\varphi, N)$-module et $g$ un élément de $(\mathcal{B}[\log x] \otimes \mathcal{D})^{N=0}$. Soit $\Delta$ le plus petit sous- $\varphi$-module de $\mathcal{D}$ tel que $g \in \mathcal{B}[\log x] \otimes \Delta$. Alors, $\Delta$ est stable par $N$.

Ainsi, $\varphi$-support et $(\varphi, N)$-support pour un élément de $(\mathcal{B}[\log x] \otimes \mathcal{D})^{N=0}$ sont identiques.

Démonstration. - Écrivons $g=\sum_{i=1}^{s} g_{i} v_{i}$ avec les $v_{i} \in \Delta$, les $g_{i} \in \mathcal{B}[\log x]$ et $s$ minimal. Comme $N g=0$, on a

$$
\sum_{i=1}^{s} g_{i} N v_{i}=-\sum_{i=1}^{s} N\left(g_{i}\right) v_{i} \in \mathcal{B}[\log x] \otimes \Delta .
$$

Dans $\mathcal{B}[\log x] \otimes \mathcal{D} / \Delta$, on a donc avec $w_{i}=N v_{i}, \sum_{i=1}^{s} g_{i} w_{i}=0$. Quitte à changer la numérotation, on peut supposer que les $w_{i}$ non nuls sont les $s_{0}$ premiers et que les $s_{1}$ premiers sont indépendants et forment une base de l'espace vectoriel engendré par les $w_{i}$. Supposons $s_{0} \neq 0$. Alors,

$$
\begin{aligned}
0 & =w_{1} \wedge \cdots \wedge w_{s_{1}-1} \wedge \sum_{i=1}^{s_{0}} g_{i} w_{i} \\
& =h w_{1} \wedge \cdots \wedge w_{s_{1}}
\end{aligned}
$$

MÉMOIRES DE LA SMF 84 
avec $h$ une combinaison linéaire non triviale des $g_{i}$ à coefficients dans $\mathbb{Q}_{p}$. Comme $h=0$, cela implique que l'un des $g_{i}$ s'exprime en fonction des autres et contredit le fait que l'écriture de $g$ est minimale. Donc, tous les $N v_{i}$ sont dans $N \Delta$. Si $\Delta^{\prime}$ est le sous-espace vectoriel de $\Delta$ engendré par les $v_{i}$, on a $\Delta=\sum \varphi^{n} \Delta^{\prime}$. Comme $N \varphi=p \varphi N$, on en déduit que $N \Delta \subset \Delta$ et le lemme.

6.1.4. Les théorèmes de stabilité de Totaro restent vrais dans le cas des $(\varphi, N)$ modules filtrés. Un $(\varphi, N)$-module filtré $\mathcal{D}$ est dit faiblement admissible si $t_{H}(\mathcal{D})=$ $t_{N}(\mathcal{D})$ et si pour tout sous-module $\mathcal{D}^{\prime}$ de $\mathcal{D}$ stable par $\varphi$ et par $N, t_{H}\left(\mathcal{D}^{\prime}\right) \leqslant t_{N}(\mathcal{D})$. Si $\mathcal{D}^{\prime}$ est un $(\varphi, N)$-module filtré non nul de dimension $d_{\mathcal{D}^{\prime}}$, posons

$$
\lambda\left(\mathcal{D}^{\prime}\right)=\left(t_{H}\left(\mathcal{D}^{\prime}\right)-t_{N}\left(\mathcal{D}^{\prime}\right)\right) / d_{\mathcal{D}^{\prime}} .
$$

Soit $c \in \mathbb{R}$. On dit qu'un $(\varphi, N)$-module filtré $\mathcal{D}$ est de pente $\leqslant c($ resp. $<c)$ si pour tout sous- $(\varphi, N)$-module filtré $\mathcal{D}^{\prime}$ non nul de $\mathcal{D}$, on a $\lambda\left(\mathcal{D}^{\prime}\right) \leqslant c\left(\right.$ resp. $\left.\lambda\left(\mathcal{D}^{\prime}\right)<c\right)$.

6.1.5. Proposition (Totaro). - Soient $\mathcal{D}_{i}$ pour $i=1,2$ deux $(\varphi, N)$-modules filtrés de pente $\leqslant c_{i}$. Alors $\mathcal{D}_{1} \otimes \mathcal{D}_{2}$ est un $(\varphi, N)$-module filtré de pente $\leqslant c_{1}+c_{2}$.

\subsubsection{Corollaire}

1) Soit $\mathcal{D}$ un $(\varphi, N)$-module filtré de pente $\leqslant c$, alors $\mathcal{D}^{\otimes n}$ et $\wedge^{n} \mathcal{D}$ sont des $(\varphi, N)$ modules filtrés de pente $\leqslant$ nc.

2) Soit $\mathcal{D}$ un $(\varphi, N)$-module filtré de pente $<0$, alors $\wedge^{n} \mathcal{D}$ est un $(\varphi, N)$-module filtré de pente $<0$.

\subsection{Esquisse de la démonstration}

6.2.1. La théorie de l'exponentielle du $\S 5$ donne une interprétation de $Z_{\infty, f}^{1}(K, V)$ similaire à celle de $[\mathbf{2 6}, \S 2.5 .3]$. Soit $\mathcal{D}$ un $(\varphi, N)$-module filtré. Soient $v$ un entier tel que $\left.\left.I_{H}(\mathcal{D}) \subset\right]-\infty, \ldots, v\right]$ et $J$ un sous-ensemble de $I_{H}(\mathcal{D})$. On note $\mathcal{A}_{v, J}^{(r)}(\mathcal{D})$ le sous- $\mathcal{H}\left(G_{\infty}\right)$-module de $\mathcal{D}_{\infty, f}(\mathcal{D})$ formé des $g$ tels que

$$
\left\{\begin{array}{l}
\mathfrak{o}_{\varphi}(g) \leqslant v+r \\
D^{-j}(g)\left(\zeta_{n}-1\right) \in K_{n} \otimes_{K} \varphi^{n} \mathrm{Fil}^{j} \mathcal{D} \text { pour } j \leqslant v \text { et } n>n_{0} \\
D^{-j}(g)\left(\zeta_{n}-1\right)=0 \text { pour } j \in J \text { et } n>n_{0} .
\end{array}\right.
$$

On pose $\mathcal{A}_{v, J}(\mathcal{D})=\mathcal{A}_{v, J}^{(0)}(\mathcal{D})$.

Ce module est plongé naturellement dans $\mathcal{A}_{v, J}^{(r), \operatorname{gros}}(\mathcal{D})$ qui est le sous- $\mathcal{B}\left(G_{\infty}\right)$ module de $\mathcal{D}_{\infty}^{\text {gros }}(\mathcal{D})$ formé des $g$ tel que

$$
\left\{\begin{array}{l}
\mathfrak{o}_{\varphi}(g) \leqslant v+r \\
D^{-j}(g)\left(\zeta_{n}-1\right) \in K_{n} \otimes_{K} \varphi^{n} \mathrm{Fil}^{j} \mathcal{D} \text { pour } j \leqslant v \text { et } n>n_{0} \\
D^{-j}(g)\left(\zeta_{n}-1\right)=0 \text { pour } j \in J \text { et } n>n_{0}
\end{array}\right.
$$


6.2.2. Proposition. - Soit $v$ le plus grand des poids de Hodge de $\mathbf{D}_{p}(V)$. Les modules $\operatorname{Frac}\left(\mathbb{Z}_{p} \llbracket G_{\infty} \rrbracket\right) \otimes Z_{\infty, J, g}^{1}(K, V)$ et $\operatorname{Frac}\left(\mathbb{Z}_{p} \llbracket G_{\infty} \rrbracket\right) \otimes \mathcal{A}_{v, J}\left(\mathbf{D}_{p}(V)\right)$ sont isomorphes.

On suppose désormais que $v=0$, c'est-à-dire que $\operatorname{Fil}^{1} \mathbf{D}_{p}(V)=0, \operatorname{Fil}^{0} \mathbf{D}_{p}(V) \neq 0$ et que $k$ est un entier négatif tel que $V$ n'admette pas de sous-représentation $W$ telle que $\mathrm{Fil}^{k} \mathbf{D}_{p}(W)=0$ : ainsi, pour tout sous-module $(\varphi, N)$-module filtré vérifiant $\mathrm{Fil}^{k} \mathcal{D}^{\prime}=0$, on a $t_{H}\left(\mathcal{D}^{\prime}\right)<t_{N}\left(\mathcal{D}^{\prime}\right)$. Nous devons montrer que $\mathcal{A}_{k}^{\text {gros }}=\mathcal{A}_{0,\{k\}}^{(0) \text {,gros }}\left(\mathbf{D}_{p}(V)\right)$ est nul.

Si l'on reprend la démonstration de [26], on est amené à considérer a priori le corps des fractions de $\mathcal{B}[\log x]$. Cependant, on remarque la chose suivante :

6.2.3. Lemme. - Soit $g \in(\mathcal{B}[\log x] \otimes \mathcal{D})^{N=0}$ non nul. Supposons qu'il existe une relation $\Phi^{r}(g)=\sum_{i=0}^{r-1} a_{i} \Phi^{i}(g)$ avec les $a_{k} \in \operatorname{Frac}(\mathcal{B}[\log x])$ n'appartenant pas tous à $\operatorname{Frac}(\mathcal{B})$. Alors, il existe une relation

$$
\Phi^{r^{\prime}}(g)=\sum_{i=0}^{r^{\prime}-1} a_{i}^{\prime} \Phi^{i}(g)
$$

avec $r^{\prime}<r$. En particulier, il existe une relation de ce type avec les $a_{i}$ dans $\operatorname{Frac}(\mathcal{B})$.

Démonstration. - On applique $N$ à la relation. En utilisant le fait que $N \Phi^{j} g=0$ pour tout $j$, on obtient :

$$
0=\sum_{i=0}^{r-1} N\left(a_{i}\right) \Phi^{i}(g) .
$$

Si $r^{\prime}$ est le plus grand entier tel que $N\left(a_{i}\right) \neq 0$ (nécessairement $0 \leqslant r^{\prime}<r$ ), on a donc

$$
\Phi^{r^{\prime}}(g)=-\sum_{i=0}^{r-1} \frac{N\left(a_{i}\right)}{N\left(a_{r^{\prime}}\right)} \Phi^{i}(g) .
$$

Il faut juste vérifier que si $a \in \operatorname{Frac}(\mathcal{B}[\log x]), N a=0$ si et seulement si $a \in \operatorname{Frac}(\mathcal{B})$, ce qui est clair (il s'agit d'une dérivation dans le corps de fractions d'une algèbre de polynômes à une variable).

Avec ces remarques, la démonstration de [26] s'adapte aisément. 


\section{APPENDICE A}

\section{DIGRESSION : LE POLYLOGARITHME}

\section{A.1. Polylogarithmes (définition naïve)}

Posons

$$
\mathfrak{L}_{0}(x)=\frac{x+1}{x}, \quad \mathfrak{L}_{1}(x)=\log x .
$$

Ce sont des éléments de $\mathcal{B}_{-}[\log x]$. On a $D\left(\mathfrak{L}_{1}\right)=\mathfrak{L}_{0}$ et ces deux fonctions vérifient

$$
\psi\left(\mathfrak{L}_{0}\right)=\mathfrak{L}_{0}, \quad \psi\left(\mathfrak{L}_{1}\right)=p^{-1} \mathfrak{L}_{1} .
$$

En effet,

$$
\begin{gathered}
\varphi \circ \psi\left(\mathfrak{L}_{0}\right)=\varphi \circ \psi\left(1+\frac{1}{x}\right)=1+\frac{1}{p} \sum_{\zeta \in \mu_{p}} \frac{1}{\zeta(1+x)-1} \\
=1+\frac{1}{(1+x)^{p}-1}=\varphi\left(\frac{1+x}{x}\right)=\varphi\left(\mathfrak{L}_{0}\right) \\
\varphi \circ \psi\left(\mathfrak{L}_{1}\right)=\frac{1}{p} \sum_{\zeta \in \mu_{p}} \log (\zeta(1+x)-1) \\
=p^{-1} \log \left((1+x)^{p}-1\right)=p^{-1} \varphi \log x=p^{-1} \varphi\left(\mathfrak{L}_{1}\right) .
\end{gathered}
$$

A.1.1. Lemme. - Il existe une unique suite $\left(\mathfrak{L}_{k}\right)_{k \in \mathbb{Z}}$ d'éléments de $\mathcal{B}[\log x]$ telle que

$$
D\left(\mathfrak{L}_{k}\right)=\mathfrak{L}_{k-1}, \quad \psi\left(\mathfrak{L}_{k}\right)=p^{-k} \mathfrak{L}_{k} .
$$

Démonstration. - Pour $k<0$, cela est clair, il faut et il suffit de prendre $\mathfrak{L}_{k}(x)=D^{-k}(1 / x)$. L'existence d'éléments $L_{k}$ de $\mathcal{B}[\log x]$ pour $k>0$ vérifiant $D\left(L_{k}\right)=L_{k-1}$ vient de la surjectivité de $D$ sur $\mathcal{B}[\log x]$. On peut alors ajuster par récurrence la constante d'intégration de manière unique pour que ces éléments vérifient $\psi\left(L_{k}\right)=p^{-k} L_{k}$. En effet, si $\psi\left(L_{k-1}\right)=p^{-(k-1)} L_{k-1}$, on a

$$
D\left(\psi\left(L_{k}\right)\right)=p^{-1} \psi\left(D\left(L_{k}\right)\right)=p^{-k} L_{k-1}=p^{-k} D\left(L_{k}\right),
$$


donc $\psi\left(L_{k}\right)-p^{-k} L_{k}=c$. En remplaçant $L_{k}$ par $L_{k}+d$, on obtient l'équation $\left(1-p^{-k}\right) d=-c$, ce qui a une solution unique si $k \neq 0$.

L'équation fonctionnelle permet ici de fixer la constante d'intégration. On aurait pu fixer la constante d'intégration par d'autres moyens. Par exemple, on peut demander, comme le fait Coleman dans [5] que la limite de $\mathfrak{L}_{k}(x)$ soit nulle lorsque $x \rightarrow(-1)^{-}$ avec $x$ restant dans une extension de $\mathbb{Q}_{p}$ de degré de ramification bornée.

Il est facile de voir que $\mathfrak{L}_{k}$ est en fait un polynôme de degré 1 en $\log x$. Pour $k=0$, $N \mathfrak{L}_{0}=0$ et $\mathfrak{L}_{0} \in \mathcal{B}$. Montrons plus précisément le lemme suivant :

A.1.2. Lemme. - Pour $k \geqslant 1$,

$$
N \mathfrak{L}_{k}(x)=\frac{\log ^{k-1}(1+x)}{(k-1) !}
$$

et $L_{k}(x)=\mathfrak{L}_{k}(x)-\left(\log ^{k-1}(1+x) /(k-1) !\right) \log x$ est un élément de $\mathcal{H}$.

On en déduit que

$$
\mathfrak{L}_{k}(x)-\frac{\log (1+x)}{k-1} \mathfrak{L}_{k-1}(x) \in \mathcal{B}
$$

Démonstration. - Faisons une récurrence sur $k$. Pour $k=1$,

$$
N \mathfrak{L}_{k}(x)=N \log x=1 \text { et } L_{1}=0 .
$$

Supposons l'hypothèse de récurrence vraie pour $k$ et écrivons

$$
\mathfrak{L}_{k}=\frac{\log ^{k-1}(1+x)}{(k-1) !} \log x+L_{k}
$$

avec $L_{k} \in \mathcal{H}$. On a à une constante près

$$
\mathfrak{L}_{k+1}=\int \frac{\mathfrak{L}_{k}}{1+x}=\frac{1}{(k-1) !} \int \frac{\log ^{k-1}(1+x) \log x}{1+x}+\int \frac{L_{k}}{1+x}
$$

(par intégration par parties)

$$
=\frac{1}{k !} \log ^{k}(1+x) \log x-\int\left(\frac{1}{k !} \frac{\log ^{k}(1+x)}{x}-\frac{L_{k}}{1+x}\right) .
$$

Le terme sous le signe intégrale est un élément de $\mathcal{H}$ car $\log (1+x) / x \in \mathcal{H}$ de même que $L_{k} /(1+x)$. Donc sa primitive qui est $L_{k+1}$ appartient à $\mathcal{H}$. On en déduit l'assertion.

A.1.3. Lemme. - Pour $k \in \mathbb{Z}, \mathfrak{L}_{k}^{(p)} \stackrel{\text { déf }}{=}\left(1-p^{-k} \varphi\right) \mathfrak{L}_{k}$ appartient à $\mathcal{B}_{-}^{\psi=0}$ et vaut 0 en $\infty$. Plus précisément, $\mathfrak{L}_{k}^{(p)}$ est défini pour $|x|>p^{-1 /(p-1)}$. 
Démonstration. - L'équation fonctionnelle et le lemme A.1.2 impliquent que

$$
\begin{aligned}
\left(1-p^{-k} \varphi\right) \mathfrak{L}_{k} & =\frac{\log ^{k-1}(1+x)}{(k-1) !}\left(1-p^{-1} \varphi\right) \log x+\left(1-p^{-k} \varphi\right) L_{k} \\
& =\frac{\log ^{k-1}(1+x)}{(k-1) !} \mathfrak{L}_{1}^{(p)}+\left(1-p^{-k} \varphi\right) L_{k} \in \mathcal{B} .
\end{aligned}
$$

Donc, $\mathfrak{L}_{k}^{(p)}=\left(1-p^{-k} \varphi\right) \mathfrak{L}_{k}$ appartient à $\mathcal{B}^{\psi=0}$. Montrons par récurrence qu'il appartient à $\mathcal{B}_{-}$et que sa valeur en $\infty$ est nulle. Cela est clair pour $\mathfrak{L}_{0}^{(p)}=1 / x-$ $1 /\left((1+x)^{p}-1\right)$. Supposons-le vrai pour $\mathfrak{L}_{k}^{(p)}$. Écrivons

$$
\mathfrak{L}_{k+1}^{(p)}=f_{-}+f_{+}
$$

avec $f_{+} \in \mathcal{H}$ et $f_{-} \in \mathcal{B}_{-}$. Comme $D$ conserve $\mathcal{H}$ et $\mathcal{B}_{-}, D\left(f_{+}\right)=0$ ce qui implique que $f_{+}$est une constante et que $\mathfrak{L}_{k}^{(p)}$ appartient à $\mathcal{B}^{-}$. Elle est donc définie en $\infty$. L'équation fonctionnelle $\psi\left(\mathfrak{L}_{k}^{(p)}\right)=0$ implique alors que $p \mathfrak{L}_{k}^{(p)}(\infty)=0$ et donc que

$$
\mathfrak{L}_{k}^{(p)}(\infty)=0 \text {. }
$$

Montrons que $\mathfrak{L}_{k}^{(p)} \in \mathcal{H}^{\left.p^{-1 /(p-1)}, \infty\right]}$. Comme $D\left(\mathfrak{L}_{k}^{(p)}\right)=\mathfrak{L}_{k-1}^{(p)}$ et qu'il s'agit d'éléments de $\mathcal{B}$, il est facile de voir que si $\mathfrak{L}_{0}^{(p)} \in \mathcal{H}^{\left[p^{-1 /(p-1)}, \infty\right]}$, il en est de même des $\mathfrak{L}_{k}^{(p)}$ pour $k \geqslant 0$. Or $1 /\left((1+x)^{p}-1\right)$ appartient à $\mathcal{H}^{\left.p^{-1 /(p-1)}, \infty\right]}$ et il en est de même pour $\mathfrak{L}_{0}^{(p)}$.

A.1.4. Coleman a montré dans [5] qu'il existe des fonctions $\mathrm{li}_{k}$ localement analytiques sur $\mathbb{P}^{1}-\{1\}$ telles que $\mathrm{li}_{k}(0)=0$ et

$$
\operatorname{li}_{0}(z)=\frac{z}{z-1}, \quad \frac{d}{d z} \operatorname{li}_{k}=\frac{\mathrm{li}_{k-1}}{z} .
$$

Reprenons rapidement sa construction. Considérons les classes résiduelles $\{|x|>1\}$ et $\{|x-a|<1\}$ pour $|a|=1$. Notons $t_{a}(x)=x-a$, ou $1 / x$ pour $a=\infty$, un paramètre local en $a$. Pour $f \in R_{a}$ avec $R_{a}$ l'espace des fonctions analytiques sur $\{|x-a|<1\}$ (ayant un développement de Taylor convergent) ou $\mathcal{B}_{a}\left[\log t_{a}(x)\right]$ où $\mathcal{B}_{a}$ est l'anneau des fonctions analytiques sur une couronne du type $\eta \leqslant t_{a}(x)<1$ (la condition au bord n'est pas nécessaire ici), l'équation différentielle $D(g)=f$ a toujours une solution. Pour chaque $a$, une puissance $\varphi^{n_{a}}$ convenable stabilise $\{|x-a|<1\}$ et il en est de même de $\psi^{n_{a}}$. Les opérateurs $\varphi^{n_{a}}$ et $\psi^{n_{a}}$ se prolongent alors à $\mathcal{B}_{a}\left[\log t_{a}(x)\right]$ (mêmes calculs que ceux faits pour $a=0$ ). Si $f$ vérifie l'équation fonctionnelle

$$
\psi^{n_{a}}(f)=p^{-k n_{a}} f
$$

l'équation différentielle $D(g)=f$ a une unique solution $g$ telle que

$$
\psi^{n_{a}}(g)=p^{-(k+1) n_{a}} g .
$$

En procédant sur chaque classe résiduelle et par récurrence, on en déduit qu'il existe une unique fonction $Z_{k}$ sur $\mathbb{C}_{p}$ privé de boules de centre $0,-1$ et $\infty$ et de rayon 
strictement inférieur à 1 (en fait $\left.p^{-1 /(p-1)}\right)$, dont la restriction à chaque boule (ou couronne) de rayon 1 est du type $R_{a}$ ou $\mathcal{B}_{a}\left[\log t_{a}(x)\right]$ et telle que

$$
D\left(Z_{k}\right)=Z_{k-1}, Z_{0}=\frac{x+1}{x}, \psi\left(Z_{k}\right)=p^{-k} Z \text {. }
$$

Si $Z_{k}^{(p)}=\left(1-p^{-k} \varphi\right) Z_{k}$, on a $\psi\left(Z_{k}^{(p)}\right)=0$ et $Z_{k}^{(p)}$ coïncide avec $\mathfrak{L}_{k}^{(p)}$ (la dérivation est bijective sur les éléments du noyau de $\psi$ sur n'importe quelle classe résiduelle). Sur la boule de centre $-1, \mathfrak{L}_{k}(x)$ coïncide avec la série $\sum_{n} t^{n} / n^{k}$ avec $t=1+x$, puisque les deux fonctions vérifient à la fois les équations différentielles et l'équation fonctionnelle $\psi=p^{-k}$ (à condition d'identifier $\log x$ et $\log (-x)$ ). Ainsi,

$$
\mathfrak{L}_{k}(x)=\operatorname{li}_{k}(1+x) .
$$

Par rapport à la méthode utilisée par Coleman, pour fixer les constantes d'intégration sur chaque classe résiduelle, nous avons juste remplacé l'évaluation sur un point de Teichmüller (fixe par une puissance de $\varphi$ ) par l'équation fonctionnelle $\psi(g)=p^{-k-1} g$ plus dans l'esprit de cet article.

Que donne le calcul explicite des primitives de $(1+x)^{-1} \mathfrak{L}_{1}$ ? On a par intégration par parties ( $C$ est une constante)

$$
\begin{aligned}
\int(1+x)^{-1} \log x-C & =\log (1+x) \log x-\int \frac{\log (1+x)}{x} \\
& =\log (1+x) \log x+\operatorname{li}_{2}(-x)
\end{aligned}
$$

D'où, sur une couronne du type $\rho \leqslant|x|<1$,

$$
\mathfrak{L}_{2}(x)=\log (1+x) \log x+\operatorname{li}_{2}(-x)+C
$$

ou encore en utilisant l'unicité du prolongement analytique

$$
\operatorname{li}_{2}(1+x)=\log (1+x) \log x+\operatorname{li}_{2}(-x)+C
$$

et en changeant $x$ en $-x$,

$$
\operatorname{li}_{2}(1-x)=\log (1-x) \log x+\operatorname{li}_{2}(x)+C
$$

Il reste à calculer la constante $C$. On déduit de l'équation fonctionnelle que

$$
\psi\left(\mathrm{li}_{2}(-x)\right)-p^{-2} \operatorname{li}_{2}(-x)=-\left(1-p^{-2}\right) C,
$$

c'est-à-dire que

$$
\sum_{\zeta \in \mu_{p}} \operatorname{li}_{2}(-\zeta(1+x)+1)-p^{-2} \operatorname{li}_{2}\left(-(1+x)^{p}+1\right)=-\left(1-p^{-2}\right) C
$$

Par prolongement analytique, cette équation reste vraie en l'infini, le premier membre est alors nul, ce qui implique que $C=0$. On trouve donc l'équation fonctionnelle classique.

Le lemme A.1.2 est en fait la proposition 7.1 de $[\mathbf{5}]$ où l'on remplace $B(0,1)$ par $B(1,1)$ (faute de frappe). 


\section{A.2. Polylogarithmes et fonction de Kubota-Leopoldt}

A.2.1. Appliquons le théorème 1.1.8 à $\mathfrak{L}_{0}^{(p)} \in \mathcal{B}^{\psi=0}$. Il existe un élément $\mathcal{L}_{K-L} \in$ $\mathcal{B}\left(G_{\infty}\right)$ tel que

$$
\mathfrak{L}_{0}^{(p)}=-\mathcal{L}_{K-L} \cdot(1+x) .
$$

En fait, $\mathcal{L}_{K-L}$ appartient à l'anneau total des fractions de $\mathbb{Z}_{p} \llbracket G_{\infty} \rrbracket$ qui est naturellement plongé dans $\mathcal{B}\left(G_{\infty}\right)$. En effet, si $\gamma$ est un générateur de $\Gamma$ (élément d'ordre infini), on a

$$
(\chi(\gamma) \gamma-1) \cdot \frac{x+1}{x} \in \mathbb{Z}_{p} \llbracket x \rrbracket .
$$

Donc $(\chi(\gamma) \gamma-1) \mathcal{L}_{K-L} \in \mathbb{Z}_{p} \llbracket G_{\infty} \rrbracket$. De même, comme $\mathfrak{L}_{k}^{(p)} \in \mathcal{B}^{\psi=0}$, il existe $f_{k} \in \mathcal{B}\left(G_{\infty}\right)$ tel que $\mathfrak{L}_{k}^{(p)}=f_{k} \cdot(1+x)$, en fait plus précisément

$$
\mathfrak{L}_{k}^{(p)}=-\mathrm{Tw}^{-k}\left(\mathcal{L}_{K-L}\right) \cdot(1+x)
$$

où Tw est l'opérateur induit par $\tau \mapsto \chi(\tau) \tau$. Il est facile de voir par (une des) construction(s) de la fonction $L p$-adique de la fonction de Kubota-Leopoldt $L_{p}(s, \eta)$ où $\eta$ est pour simplifier un caractère d'ordre fini de $G_{\infty}$, que l'on a

$$
\mathcal{L}_{K-L}\left(\eta\langle\chi\rangle^{s}\right)=L_{p}(-s, \eta \omega)
$$

pour $s \in \mathbb{Z}_{p}-\{1\}$ avec $\omega$ le caractère de Teichmüller de $\Delta$ tel que $\langle\chi\rangle=\chi \omega^{-1}$ soit à valeurs dans $1+p \mathbb{Z}_{p}$. Ainsi pour $k \in \mathbb{Z}$,

$$
\mathcal{L}_{K-L}\left(\eta \chi^{k}\right)=L_{p}\left(-k, \eta \omega^{k+1}\right) .
$$

On en déduit les formules classiques reliant $L_{p}\left(k, \eta \omega^{1-k}\right)$ avec la fonction $\mathfrak{L}_{k}^{(p)}$ pour $\eta$ un caractère non trivial primitif de conducteur une puissance $p^{n_{\eta}}$ de $p$ pour simplifier $[\mathbf{2 0}, 3]$ :

$$
\begin{aligned}
G\left(\eta^{-1}, \zeta_{n_{\eta}}\right) \mathcal{L}_{K-L}\left(\eta \chi^{-k}\right) & =\sum_{\tau \in \operatorname{Gal}\left(K_{n_{\eta}} / \mathbb{Q}_{p}\right)} \eta(\tau)^{-1} \tau D^{-k}\left(\mathfrak{L}_{0}^{(p)}\right)\left(\zeta_{n_{\eta}}-1\right) \\
& =\sum_{\tau \in \operatorname{Gal}\left(K_{n_{\eta}} / \mathbb{Q}_{p}\right)} \eta(\tau)^{-1} \mathfrak{L}_{k}^{(p)}\left(\zeta_{n_{\eta}}^{\tau}-1\right)
\end{aligned}
$$

avec $G\left(\eta^{-1}, \zeta_{n_{\eta}}\right)=\sum_{\tau \in \operatorname{Gal}\left(K_{n_{\eta}} / \mathbb{Q}_{p}\right)} \eta(\tau) \zeta_{n_{\eta}}^{\tau}$ et donc

$$
L_{p}\left(k, \eta \omega^{1-k}\right)=G\left(\eta^{-1}, \zeta_{n_{\eta}}\right)^{-1} \sum_{\tau \in \operatorname{Gal}\left(K_{n_{\eta}} / \mathbb{Q}_{p}\right)} \eta(\tau)^{-1} \mathfrak{L}_{k}^{(p)}\left(\zeta_{n_{\eta}}^{\tau}-1\right) .
$$

La primitivité du caractère $\eta$ permet alors de remplacer $\mathfrak{L}_{k}^{(p)}\left(\zeta_{n}-1\right)$ par $\mathfrak{L}_{k}\left(\zeta_{n}-1\right)$ :

$$
L_{p}\left(k, \eta \omega^{1-k}\right)=G\left(\eta^{-1}, \zeta_{n_{\eta}}\right)^{-1} \sum_{\tau \in \operatorname{Gal}\left(K_{n_{\eta}} / \mathbb{Q}_{p}\right)} \eta(\tau)^{-1} \mathfrak{L}_{k}\left(\zeta_{n_{\eta}}^{\tau}-1\right) .
$$

La formule de Coleman

$$
\mathfrak{L}_{k}(x)=\operatorname{li}_{k}(1+x)
$$


pour tout $x$ tel que $|x| \geqslant p^{-1 /(p-1)}$ permet alors d'écrire cette formule sous la forme voulue

$$
L_{p}\left(k, \eta \omega^{1-k}\right)=G\left(\eta^{-1}, \zeta_{n_{\eta}}\right)^{-1} \sum_{\tau \in \operatorname{Gal}\left(K_{n_{\eta}} / \mathbb{Q}_{p}\right)} \eta(\tau)^{-1} \operatorname{li}_{k}\left(\zeta_{n_{\eta}}^{\tau}\right) .
$$

Cependant, la formule fondamentale est :

$$
\mathfrak{L}_{k}\left(\zeta_{n}-1\right)=\operatorname{li}_{k}\left(\zeta_{n}\right)=\sum_{m>0} \frac{\zeta_{n}^{m}}{m^{k}}
$$

La formule pour le caractère trivial s'en déduit de la manière suivante. Comme $\psi\left(\mathcal{L}_{k}\right)=p^{-k} \mathcal{L}_{k}$, il est naturel de poser :

$$
\left[\left(1-p^{1-k} \varphi\right) \mathcal{L}_{k}\right](0)=-\operatorname{Tr}_{K_{1} / \mathbb{Q}_{p}}\left(\mathcal{L}_{k}\left(\zeta_{1}-1\right)\right)
$$

Si $\left(1-p^{-k} \varphi\right) G=1+x-\log ^{k}(1+x), G(0)$ est défini et vaut $\left(1-p^{-k}\right)^{-1}$ et on a

$$
\left.\left(1-p^{1-k}\right) G(0)=\left[\left(1-p^{1-k}\right) \varphi\right) G\right](0)=-\operatorname{Tr}_{K_{1} / \mathbb{Q}_{p}}\left(G\left(\zeta_{1}-1\right)\right)
$$

d'une part et

$$
\mathrm{Tw}^{-k}\left((\chi(\gamma) \gamma-1) \mathcal{L}_{K-L}\right) \cdot G=\left(\chi^{1-k}(\gamma) \gamma-1\right) \mathfrak{L}_{k}
$$

d'autre part. On en déduit que

$$
\mathcal{L}_{K-L}\left(\chi^{-k}\right)\left(1-p^{1-k}\right) G(0)=\left[\left(1-p^{1-k} \varphi\right) \mathcal{L}_{k}\right](0)
$$

ou encore

$$
\mathcal{L}_{K-L}\left(\chi^{-k}\right)=\left(1-p^{-k}\right) \frac{\left[\left(1-p^{1-k} \varphi\right) \mathcal{L}_{k}\right](0)}{1-p^{1-k}} .
$$

On pose $\mathcal{L}_{k}(0)=\left[\left(1-p^{1-k} \varphi\right) \mathcal{L}_{k}\right](0) /\left(1-p^{1-k}\right)$. En utilisant l'équation

$$
\left[\left(1-p^{1-k} \varphi\right) \mathcal{L}_{k}\right](x)=-\operatorname{Tr}_{K_{1} / \mathbb{Q}_{p}}\left(\mathcal{L}_{k}\left(\zeta_{1}(1+x)-1\right)\right)
$$

on peut interpréter comme le fait Coleman $\mathcal{L}_{k}(0)$ comme la limite de $\mathcal{L}_{k}(x)$ pour $x$ tendant vers 0 dans une extension de $\mathbb{Q}_{p}$ de degré de ramification borné. On retrouve la formule (4) de [5]

$$
\mathcal{L}_{K-L}\left(\chi^{-k}\right)=\left(1-p^{-k}\right) \mathcal{L}_{k}(0)
$$

Nous ne prétendons pas avoir fait plus simple que [5]! La théorie du prolongement analytique de Coleman est fondamentale pour reconnaître en $\mathfrak{L}_{k}\left(\zeta_{n}-1\right)$ le nombre $\operatorname{li}_{k}\left(\zeta_{n}\right)$ (remarquons cependant que la construction de $\mathfrak{L}_{k}$ est dans un certain sens plus facile $\left.{ }^{(1)}\right)$, nous avons simplement remplacé l'argument qui utilise la construction de Koblitz $([\mathbf{1 4}])$ de la fonction $L$ p-adique utilisant les mesures sur $\mathbb{Z}_{p}^{\times}$par la construction basée sur les unités cyclotomiques et la série $(x+1) / x^{(2)}$. Par contre la méthode

\footnotetext{
${ }^{(1)}$ pourquoi d'ailleurs préfère-t-on avoir la singularité en 1 plutôt qu'en 0 ? et pourquoi les intégrales itérées de $\log (1-x) / x$ ont-elles plus de prestige que celles de $\log (x) /(1+x)$ ?

${ }^{(2)}$ Finalement, l'étude de la fonction $L p$-adique de Kubota-Leopoldt est simplement l'étude de $x^{-1}$ et de sa primitive $\log x$ ! Ce qui traduit aussi la simplicité du système d'Euler associé qui est $\left(1-\zeta_{n}\right)_{n}$.
} 
semble se généraliser au cas des courbes elliptiques à réduction multiplicative déployée par exemple pour l'étude du zéro trivial.

A.2.2. Ce paragraphe est lié au paragraphe 5. Prenons

$$
V=\mathbb{Q}_{p}(1) \text { et } g=\mathfrak{L}_{1}^{(p)} \otimes e_{-1} \in \mathbb{Q}_{p}[-1]=\mathbf{D}_{p}\left(\mathbb{Q}_{p}(1)\right)
$$

et

$$
G=\log x \otimes e_{-1} .
$$

L'action de $\varphi$ sur $\mathbb{Q}_{p}[-1]$ est la multiplication par $p^{-1}$. On a $(1-\Phi) G=g$. Comme ni 1 ni $p^{-1}$ ne sont valeurs propres de $\varphi$ sur $\mathbb{Q}_{p}[-k]$ pour $k \geqslant 2$ et que $N G=e_{-1}$, on a $\Lambda(G)=e_{-1}, \mathfrak{M}(G)=0$. Traduisons le théorème 5.3.5. Comme

$$
\mathrm{Fil}^{-1} \mathbf{D}_{p}\left(\mathbb{Q}_{p}(1)\right)=\mathbf{D}_{p}\left(\mathbb{Q}_{p}(1)\right),
$$

prenons $h=1$. Les points $P_{n, 0}^{(k)}=P_{n, 0}^{(k)}\left(\log x \otimes e_{-1}\right)$ sont

$$
\begin{aligned}
P_{n, 0}^{(1)} & =\exp _{\mathbb{Q}_{p}(1)}\left(p^{-n}(1 \otimes \varphi)^{-n}\left(p^{-n} \Lambda, 0, G\right)\left(\zeta_{n}-1\right)\right) \\
& =\exp _{\mathbb{Q}_{p}(1)}\left(\left(p^{-n}, 0, \log \left(\zeta_{n}-1\right) e_{-1}\right)\right. \\
& =\zeta_{n}-1
\end{aligned}
$$

et pour $k \geqslant 1$,

$$
\begin{aligned}
P_{n, k}^{(1)} & =\exp _{\mathbb{Q}_{p}(k+1)}\left(p^{n(k-1)}(1 \otimes \varphi)^{-n}\left(0,0, D^{-k}(G)\left(\zeta_{n}-1\right)\right)\right) \\
& =\exp _{\mathbb{Q}_{p}(k+1)}\left(p^{n k}\left(0,0, \mathfrak{L}_{k+1}\left(\zeta_{n}-1\right)\right) e_{-k-1}\right)
\end{aligned}
$$

Par le théorème 5.3.5,

$$
(-1)^{k-1} k ! p^{n k} P_{n, k}^{(1)}=\exp _{\mathbb{Q}_{p}(k+1), e}\left((-1)^{k-1} k ! p^{n k} \mathfrak{L}_{k+1}\left(\zeta_{n}-1\right) e_{-k-1}\right)
$$

est aussi l'image dans $H^{1}\left(K_{n}, \mathbb{Q}_{p}(k+1)\right)$ du twist $\operatorname{Tw}^{k}\left(\left(1-\zeta_{m}\right)_{m}\right) \in Z_{\infty}^{1}\left(K, \mathbb{Q}_{p}(k+1)\right)$ du système des éléments cyclotomiques $\left(1-\zeta_{m}\right)_{m}$. Autrement dit, pour $k \geqslant 2$

$$
p^{n(k-1)} \mathfrak{L}_{k}\left(\zeta_{n}-1\right) e_{-k}=\Gamma^{*}(-k+1) \log _{\mathbb{Q}_{p}(k)}\left(\pi_{n}^{(k)}\left(\operatorname{Tw}^{k-1}\left(\left(1-\zeta_{m}\right)_{m}\right)\right)\right)
$$

où $\pi_{n}^{(k)}$ est la projection de $\lim _{n} H^{1}\left(K_{m}, \mathbb{Z}_{p}(k)\right)$ sur $H^{1}\left(K_{n}, \mathbb{Z}_{p}(k)\right)$ et où $\mathrm{Tw}^{k}$ est cette fois l'opérateur de twist (dû à Soulé dans ce cas) de $\varliminf_{n} H^{1}\left(K_{n}, \mathbb{Z}_{p}\right)$ dans $\lim _{n} H^{1}\left(K_{n}, \mathbb{Z}_{p}(k)\right)$. D'où en utilisant le polylogarithme li $_{k}$ de Coleman et par simple application du théorème principal,

$$
\operatorname{li}_{k}\left(\zeta_{n}\right)=\frac{\Gamma^{*}(-k+1)}{p^{n(k-1)}} \log _{\mathbb{Q}_{p}(k)}\left(\pi_{n}^{(k)} \operatorname{Tw}^{k-1}\left(1-\zeta_{n}\right)_{m}\right) .
$$

Lorsque $k<0$, on obtient de même les formules

avec

$$
\operatorname{li}_{k}\left(\zeta_{n}\right)=\frac{\Gamma^{*}(-k+1)}{p^{n(k-1)}} \exp _{\mathbb{Q}_{p}(k)}^{*}\left(\pi_{n}^{(k)} \mathrm{Tw}^{k-1}\left(1-\zeta_{n}\right)_{m}\right)
$$

$$
\operatorname{li}_{k}(z)=\left(z \frac{d}{d z}\right)^{-k} \frac{z}{(z-1)}
$$


et $\exp _{\mathbb{Q}_{p}(k)}^{*}$ l'exponentielle duale. Ce genre de formules et ses généralisations sont certainement la motivation secrète fondamentale de tout ce travail.

\section{A.3. Polylogarithmes et éléments de $B_{\mathrm{st}}^{+} G_{K_{\infty}}$}

A.3.1. Reformulons dans ce cas particulier les propositions 5.1.4 et 5.1.7.

Proposition. - La suite $p^{n k} \mathfrak{L}_{k}\left(\beta_{n}-1\right)=p^{n k} \operatorname{li}_{k}\left(\beta_{n}\right)$ converge dans $B_{\mathrm{dR}}$ vers un élément $\mathfrak{L}_{k, \mathrm{st}}$ de $B_{\mathrm{st}}^{+G_{K_{\infty}}}$ vérifiant

$$
\begin{aligned}
\varphi \mathfrak{L}_{k, \mathrm{st}} & =p^{k} \mathfrak{L}_{k, \mathrm{st}}, \quad N \mathfrak{L}_{k, \mathrm{st}}=\frac{t^{k-1}}{(k-1) !} \\
p^{n} T_{n}\left(\mathfrak{L}_{k, \mathrm{st}}\right) & =p^{n k} \mathfrak{L}_{k}\left(\beta_{n}-1\right)=\sum_{j \leqslant k} p^{n j} \operatorname{li}_{j}\left(\zeta_{n}\right) \frac{t^{k-j}}{(k-j) !} .
\end{aligned}
$$

Avec les notations du $§ 5.1 .4$, on a

$$
\mathcal{L}_{\mathbb{Q}_{p}[-k]}\left(\mathfrak{L}_{k} \otimes e_{-k}\right)=\mathfrak{L}_{k, \mathrm{st}} \otimes e_{-k} .
$$

Les $\mathfrak{L}_{k, \text { st }}$ sont les avatars de la fonction de Kubota-Leopoldt dans $B_{\text {st }}$.

A.3.2. Soit le polylogarithme de Debye (ou plutôt une translatée)

$$
\mathfrak{D}_{k}=\sum_{j=0}^{k-1}(-1)^{j} \frac{\log ^{j}(1+x)}{j !} \mathfrak{L}_{k-j} .
$$

Proposition

1) La dérivée de $\mathfrak{D}_{k}$ est

$$
\mathfrak{D}_{k}^{\prime}(x)=\frac{(-1)^{k-1}}{(k-1) !} \frac{\log ^{k-1}(1+x)}{x} .
$$

Pour $k \geqslant 2, \mathfrak{D}_{k}$ appartient à $\mathcal{H}$. Pour $k=1, \mathfrak{D}_{k}=\log x$.

2) Pour $n \gg 0$, les $\mathfrak{D}_{k}\left(\beta_{n}-1\right)$ appartiennent $\grave{a} B_{\text {cris }}^{+G_{K_{\infty}}}$ et

$$
\mathfrak{D}_{k}\left(\beta_{n}-1\right) \equiv \mathfrak{L}_{k}\left(\zeta_{n}-1\right) \bmod \mathrm{Fil}^{k} B_{\mathrm{dR}}^{+} .
$$

3) La suite $p^{n k} \mathfrak{D}_{k}\left(\beta_{n}-1\right)$ converge vers un élément $\mathfrak{D}_{k, \mathrm{st}}$ de $B_{\text {cris }}^{+G_{K_{\infty}}}$ pour $k \geqslant 2$ (resp. de $B_{\mathrm{st}}^{+G_{K_{\infty}}}$ pour $k=1$ ) vérifiant $\varphi\left(\mathfrak{D}_{k, \mathrm{st}}\right)=p^{k} \mathfrak{D}_{k, \mathrm{st}}$. On a enfin

$$
\begin{aligned}
p^{n} T_{n}\left(\mathfrak{D}_{k, \mathrm{st}}\right) & =p^{n k} \mathfrak{D}_{k}\left(\beta_{n}-1\right) \\
& =p^{n k} \mathfrak{L}_{k}\left(\zeta_{n}-1\right)+(-1)^{k-1} \sum_{j \geqslant 0} p^{-n j} \mathfrak{L}_{-j}\left(\zeta_{n}-1\right) \frac{t^{j+k}}{(j+k) !} .
\end{aligned}
$$

Avec les notations du $\S 5.1 .4$, on a

$$
\mathcal{L}_{\mathbb{Q}_{p}[-k]}\left(\mathfrak{D}_{k} \otimes e_{-k}\right)=\mathfrak{D}_{k, \mathrm{st}} \otimes e_{-k} ;
$$


On a aussi

$$
\mathfrak{D}_{k, \mathrm{st}}=\sum_{j=0}^{k-1} \mathfrak{L}_{k-j, \mathrm{st}} \frac{t^{j}}{j !}
$$

La dernière formule de la proposition peut aussi s'écrire pour $n \geqslant 1$

$$
\begin{aligned}
p^{n} T_{n}\left(\mathfrak{D}_{k, \mathrm{st}}\right) & =\sum_{j \leqslant k} p^{n j} \mathfrak{D}_{j}\left(\zeta_{n}-1\right) \frac{t^{k-j}}{(k-j) !} \\
& =\sum_{j \leqslant k} p^{n j} \operatorname{li}_{j}\left(\zeta_{n}\right) \frac{t^{k-j}}{(k-j) !} .
\end{aligned}
$$

Démonstration. - Pour $k=1, \mathfrak{D}_{1}=\log x$. Supposons maintenant $k \geqslant 2$. On a

$$
\begin{aligned}
N \mathfrak{D}_{k} & =\sum_{j=0}^{k-1} \frac{\log ^{j}(1+x)}{j !} N \mathfrak{D}_{k-j} \\
& =\sum_{j=0}^{k-1} \frac{(-1)^{j}}{j !(k-1-j) !} \log ^{k-1}(1+x)=0 .
\end{aligned}
$$

Donc $\mathfrak{D}_{k} \in \mathcal{B}$. En utilisant le lemme A.1.2, on montre facilement que $\mathfrak{D}_{k} \in \mathcal{H}$. On retrouve ce résultat grâce au calcul de la dérivée

$$
D\left(\mathfrak{D}_{k}\right)=(-1)^{k-1} \frac{\log ^{k-1}(1+x)}{k-1 !} \mathfrak{L}_{0}=(-1)^{k-1} \frac{\log ^{k-1}(1+x)}{k-1 !}\left(1+\frac{1}{x}\right)
$$

ou

$$
\mathfrak{D}_{k}^{\prime}=\frac{(-1)^{k-1}}{(k-1) !} \frac{\log ^{k-1}(1+x)}{x}
$$

D'autre part,

$$
\left(1-p^{-k} \varphi\right) \mathfrak{D}_{k}=\sum_{j=0}^{k-1}(-1)^{j} \frac{\log ^{j}(1+x)}{j !} \mathfrak{L}_{k-j}^{(p)}
$$

est d'ordre $\leqslant k-1$. La convergence de la suite des $p^{n k} \mathfrak{D}_{k}\left(\beta_{n}-1\right)$ se déduit alors du lemme 5.1.5. Pour $k=1, \mathfrak{D}_{1, \text { st }}$ est égal à $\log ([\varepsilon-1])$.

Pour la dernière formule, $D^{r}\left(\mathfrak{D}_{k}\right)\left(\right.$ resp. $\left.D^{r}\left(\mathfrak{D}_{k}\right)-(-1)^{k-1} D^{r-k}\left(\mathfrak{L}_{0}\right)\right)$ est divisible par $\log (1+x)$ si $r \leqslant k-1$ (resp. si $r \geqslant k$ ). Comme $D^{j}\left(\mathfrak{L}_{0}\right)=\mathfrak{L}_{-j}$, on en déduit la formule.

Les éléments $\mathfrak{D}_{k, \text { st }}$ interviennent de manière essentielle dans la construction de l'application $\Omega_{\mathbb{Q}_{p}(k)}$ (de manière un peu plus cachée ici que dans [19] et [25]) lorsque l'on cherche à relever un élément $\beta$ de $K_{n}$ en un élément $\widehat{\beta}$ de $B_{\text {cris }}^{+G_{K_{\infty}}}$ de manière à ce que $\widehat{\beta} \equiv \beta \bmod \mathrm{Fil}^{h} B_{\mathrm{dR}}$ pour $h$ imposé à l'avance. C'est le cas de $\mathfrak{D}_{k}\left(\beta_{n}-1\right)$ et de 
$\mathfrak{L}_{k}\left(\zeta_{n}-1\right)=\operatorname{li}_{k}\left(\zeta_{n}\right)$. En passant à la limite, on obtient un élément $\mathfrak{D}_{k, \mathrm{st}}$ de $B_{\text {cris }}^{+G_{K_{\infty}}}$ vérifiant $\varphi \mathfrak{D}_{k, \text { st }}=p^{k} \mathfrak{D}_{k, \text { st }}$ et tel que

$$
T_{n}\left(\mathfrak{D}_{k, \mathrm{st}}\right) \equiv p^{n(k-1)} \mathfrak{L}_{k}\left(\zeta_{n}-1\right) \bmod \mathrm{Fil}^{k} B_{\mathrm{dR}}^{+} .
$$

C'est cela «l'avantage » de $\mathfrak{D}_{k, \text { st }}$ sur $\mathfrak{L}_{k, \text { st }}$. 


\section{APPENDICE B}

\section{ÉTUDE DE $\mathcal{B}^{\psi=0}$}

Le but de cet appendice est de montrer le théorème 1.1.8 de Colmez-Cherbonnier. La démonstration est reprise de [3].

\section{B.1. Préliminaires}

Dans ce qui suit, les réels $\rho, \rho^{\prime}$ vérifient sauf avis contraire $0 \leqslant \rho \leqslant \rho^{\prime} \leqslant 1$.

B.1.1. LEMME

1) Soit $f=\sum_{n \in \mathbb{Z}} a_{n} x^{n}$ un élément de $\mathcal{H}^{\left[\rho, \rho^{\prime}\right)}$. S'il existe un entier $n_{0} \in \mathbb{Z}$ tel que

$$
\left|a_{n}\right| r^{n}<\left|a_{n_{0}}\right| r^{n_{0}}
$$

pour tout $n \neq n_{0}$ et pour tout $\rho \leqslant r \leqslant \rho^{\prime}$, alors $f / x^{n_{0}}$ est inversible dans $\mathcal{H}^{\left[\rho, \rho^{\prime}\right)}$. Si de plus, $\left|a_{n_{0}}\right|=1$ (c'est-à-dire $\|f\|_{1}=1$ ), alors $f$ est inversible dans $\mathcal{H}_{0}^{\left[\rho, \rho^{\prime}\right)}$.

2) Soit $f=\sum_{n \in \mathbb{Z}} a_{n} x^{n}$ un élément de $\mathcal{H}^{\left[\rho, \rho^{\prime}\right)}$ (resp. $\left.\mathcal{H}^{\left[\rho, \rho^{\prime}\right]}\right)$. On suppose que $f$ n'a pas de zéros pour $\rho \leqslant|x|<1$ (resp. $\rho \leqslant|x| \leqslant 1$ ). Alors $f$ est inversible dans $\left.\mathcal{H}^{\left[\rho, \rho^{\prime}\right.}\right)$ (resp. $\left.\mathcal{H}^{\left[\rho, \rho^{\prime}\right]}\right)$.

Démonstration. — voir [1].

La première condition implique en particulier que $\left|a_{n}\right| \leqslant\left|a_{n_{0}}\right|$ pour tout $n$.

\section{B.2. Structure de $G_{\infty}$-modules}

On fixe $\tau \in \Gamma$, on pose $\chi(\tau)=1+p^{m} u$ avec $u$ unité. On fixe $\rho<1$. On pose $\rho_{m}=p^{-1 /\left((p-1) p^{m}\right)}$.

B.2.1. Lemme. - $(\tau-1)(x) / x^{p^{m}}$ est une unité de $\mathcal{H}_{0}^{[\rho, 1)}$ pour $\rho>\rho_{m-1}$. De même, $\varphi(x) / x^{p}$ est une unité de $\mathcal{H}_{0}^{[\rho, 1)}$ pour $\rho>\rho_{0}$. 
Démonstration. - On a

$$
\begin{aligned}
(\tau-1)(x) & =(1+x)\left[(1+x)^{u p^{m}}-1\right] \\
& =(1+x) \sum_{k=1}^{\infty}\left(\begin{array}{c}
p^{m} u \\
k
\end{array}\right) x^{k}=(1+x) \sum_{n \geqslant 1} a_{n} x^{n} .
\end{aligned}
$$

Il s'agit de montrer que $\sum_{n} a_{n} x^{n} / x^{p^{m}}$ est inversible. On utilise le lemme B.1.1 avec $n_{0}=p^{m}$. D'abord, $a_{p^{m}}=\left(\begin{array}{c}p^{m} u \\ p^{m}\end{array}\right)$ est une unité car $u$ est premier à $p$. Soit $\rho \leqslant r<1$. Pour $n>p^{m}$, on a $\left|a_{n}\right| r^{n} \leqslant r^{n} \leqslant r^{p^{m}}$. Pour $n<p^{m}$, on a

$$
\left|a_{n}\right| r^{n}=\left\|\left(\begin{array}{c}
p^{m} u \\
n
\end{array}\right)\right\| r^{n}=p^{-m+\operatorname{ord}_{p} n} r^{n} .
$$

On désire montrer que pour $n<p^{m}$,

$$
p^{-m+\operatorname{ord}_{p} n} r^{n}<r^{p^{m}}
$$

Il suffit pour cela de le vérifier pour $n$ de la forme $p^{m^{\prime}}$ avec $m^{\prime}<m$, c'est-à-dire de vérifier que pour $m^{\prime}<m$,

$$
p^{m^{\prime}} r^{p^{m^{\prime}}}<p^{m} r^{p^{m}}
$$

Or la fonction $x \mapsto p^{x} r^{p^{x}}$ est croissante puis décroissante, il suffit donc de vérifier que

$$
p^{m-1} r^{p^{m-1}}<p^{m} r^{p^{m}}
$$

ce qui est vrai si et seulement si $r^{p^{m-1}(p-1)}>p^{-1}$, c'est-à-dire si et seulement si $r>\rho_{m-1}$. Pour $\varphi(x) / x^{p}=\sum_{j=1}^{p-1}\left(\begin{array}{c}p \\ j\end{array}\right) x^{j-p}$, on a pour tout $j \neq p,\left|\left(\begin{array}{l}p \\ j\end{array}\right) r^{j-p}\right|<p^{-1} r^{1-p}$, ce qui est strictement inférieur à 1 pour $r>p^{-1 /(p-1)}$.

B.2.2. LEMME. - Pour tout entier $k \in \mathbb{Z},(\tau-1)\left(x^{k}\right) / x^{k}$ appartient à $x^{p^{m}-1} \mathcal{H}_{0}^{[\rho, 1)}$ pour $\rho>\rho_{m-1}$.

Démonstration. - Si $k=1$, c'est le lemme B.2.1. Si $k \neq 1$, le lemme se déduit du cas $k=1$ et de

$$
\frac{(\tau-1)\left(x^{k}\right)}{x^{k}}=\sum_{j=1}^{\infty}\left(\begin{array}{l}
k \\
j
\end{array}\right)\left(\frac{(\tau-1) x}{x}\right)^{j} .
$$

B.2.3. Lemme. - Prenons $\}=)$, ]. Si $\rho^{\prime} \geqslant \rho>\rho_{m-1}$, l'image de $x^{a} \mathcal{H}_{0}^{\left[\rho, \rho^{\prime}\right\}}$ par $\tau-1$ est contenue dans $x^{a+p^{m}-1} \mathcal{H}_{0}^{\left[\rho, \rho^{\prime}\right\}}$.

Démonstration. - Comme $\mathcal{H}_{0}^{\left[\rho, \rho^{\prime}\right\}}$ est complet, il suffit de démontrer cette propriété sur un sous-ensemble dense. Si $\beta \in \mathbb{Q}_{p}, \beta x^{s}$ est un élément de $\mathcal{H}_{0}^{\left[\rho, \rho^{\prime}\right]}$ si et seulement si $|\beta| \rho^{s} \leqslant 1$ et $|\beta| \rho^{\prime s} \leqslant 1$. Le sous- $\mathbb{Z}_{p}$-module de $\mathcal{H}_{0}^{\left[\rho, \rho^{\prime}\right\}}$ engendré par de tels éléments est dense dans $\mathcal{H}_{0}^{\left[\rho, \rho^{\prime}\right\}}$. On a alors

$$
(\tau-1)\left(\beta x^{a+s}\right)=v \beta x^{s+a+p^{m}-1}=x^{a+p^{m}-1} v \beta x^{s} \in x^{a+p^{m}-1} \mathcal{H}_{0}^{\left[\rho, \rho^{\prime}\right]}
$$

avec $v \in \mathcal{H}_{0}^{[\rho, 1)} \subset \mathcal{H}_{0}^{\left[\rho, \rho^{\prime}\right]}$. 
Remarque. - On a $(\tau-1)(x)=x^{p^{m}} U$ avec $U$ une unité d'après le lemme B.2.1 . Donc $(\tau-1) \varphi(x)=x^{p^{m+1}} U^{\prime}$.

B.2.4. Proposition (Colmez-Cherbonnier). - Soit $\rho_{m}<\rho<\rho^{\prime}$. Pour tout $a \in \mathbb{Z}$, $\tau-1$ est une injection

$$
\varphi(x)^{a} \mathcal{H}_{0}^{\left[\rho, \rho^{\prime}\right)^{\psi=0}} \longrightarrow \varphi(x)^{a+p^{m-1}-1} \mathcal{H}_{0}^{\left[\rho, \rho^{\prime}\right)^{\psi=0}}
$$

dont l'image contient $\varphi(x)^{a+p^{m-1}} \mathcal{H}_{0}^{\left[\rho, \rho^{\prime}\right)^{\psi=0}}$.

B.2.5. Corollaire. - Soit $\rho>\rho_{m}$. Pour tout $a \in \mathbb{Z}, \tau-1$ induit une bijection de

$$
\mathcal{H}^{\left[\rho, \rho^{\prime}\right)^{\psi=0}} \longrightarrow \mathcal{H}^{\left[\rho, \rho^{\prime}\right)} \psi=0
$$

et on a

$$
(\tau-1)^{-1}\left(\varphi(x)^{a} \mathcal{H}_{0}^{\left[\rho, \rho^{\prime}\right)^{\psi=0}}\right) \subset \varphi(x)^{a-p^{m-1}} \mathcal{H}_{0}^{\left[\rho, \rho^{\prime}\right)^{\psi=0}}
$$

Démonstration. - Reprenons la démonstration de [3]. Un élément de $\mathcal{H}_{0}^{\left[\rho, \rho^{\prime}\right)^{\psi=0}}$ s'écrit de manière unique sous la forme

$$
g=\sum_{i=1}^{p-1}(1+x)^{i} \varphi\left(g_{i}\right)
$$

avec les $g_{i} \in \mathcal{H}^{\left[\rho^{1 / p}, \rho^{1 / p}\right)}$. En utilisant le fait que $\varphi(x) / x^{p}$ est une unité et le lemme B.2.2, on obtient

$$
\begin{aligned}
(\tau-1)\left(\varphi(x)^{a}(x+1)^{i} \varphi(g)\right) & \in(\tau-1)\left(x^{a p} \mathcal{H}_{0}^{\left[\rho, \rho^{\prime}\right)}\right) \\
& \subset x^{a p+p^{m}-1} \mathcal{H}_{0}^{\left[\rho, \rho^{\prime}\right)}=\varphi(x)^{a+p^{m-1}-1} x^{p-1} \mathcal{H}_{0}^{\left[\rho, \rho^{\prime}\right)} \\
& \subset \varphi(x)^{a+p^{m-1}-1} \mathcal{H}_{0}^{\left[\rho, \rho^{\prime}\right)}
\end{aligned}
$$

et donc

$$
(\tau-1)\left(\varphi(x)^{a} \mathcal{H}_{0}^{\left[\rho, \rho^{\prime}\right)^{\psi=0}}\right) \subset \varphi(x)^{a+p^{m-1}-1} \mathcal{H}_{0}^{\left[\rho, \rho^{\prime}\right)^{\psi=0}} .
$$

Construisons maintenant un «presque inverse» de $\tau-1$ par la formule

$$
\mu(g)=\sum_{i=1}^{p-1}(1+x)^{i} \frac{\varphi\left(g_{i}\right)}{(x+1)^{i(\chi(\tau)-1)}-1} .
$$

On a

$$
\mu(g)=\sum_{i=1}^{p-1}(1+x)^{i} \frac{\varphi\left(u_{i} g_{i}\right)}{\varphi(x)^{p^{m-1}}} .
$$


où $u_{i}=-x^{p^{m-1}} /\left((x+1)^{-i(\chi(\tau)-1) / p}-1\right)$ est une unité de $\mathcal{H}_{0}^{\left[\rho, \rho^{\prime}\right)}$. Si $g \in \varphi(x)^{\left.a \mathcal{H}_{0}^{[} \rho, \rho^{\prime}\right)^{\psi=0}}, \mu(g)$ appartient à $\varphi(x)^{a-p^{m-1}} \mathcal{H}_{0}^{\left[\rho, \rho^{\prime}\right)^{\psi=0}}$. On a d'autre part

$$
\begin{aligned}
\mu((\tau-1)(g)) & =g-\sum_{i=1}^{p-1}(1+x)^{i} \varphi\left(\frac{(\tau-1)\left(g_{i}\right)}{(x+1)^{-i(\chi(\tau)-1) / p}-1}\right) \\
& =\sum_{i=1}^{p-1}(1+x)^{i} \varphi\left(u_{i} \frac{(\tau-1)\left(g_{i}\right)}{x^{p^{m-1}}}\right) .
\end{aligned}
$$

En particulier, si $g \in \varphi(x)^{a} \mathcal{H}_{0}^{\left[\rho, \rho^{\prime}\right)^{\psi=0}}$ pour $a \in \mathbb{Z}$ et pour $\rho \geqslant \rho_{m}, \mu((\tau-1)(g))-g$ est un élément de

$$
\varphi(x)^{a+p^{m}-1-p^{m-1}} \mathcal{H}_{0}^{\left[\rho, \rho^{\prime}\right)}=\varphi(x)^{a+p^{m-1}(p-1)-1} \mathcal{H}_{0}^{\left[\rho, \rho^{\prime}\right)} \subset \varphi(x)^{a+1} \mathcal{H}_{0}^{\left[\rho, \rho^{\prime}\right)}
$$

(on utilise ici le lemme B.2.2). Montrons que $\varphi(x)^{a+p^{m-1}} \mathcal{H}_{0}^{\left[\rho, \rho^{\prime}\right)^{\psi=0}}$ est contenu dans l'image de $\varphi(x)^{a} \mathcal{H}_{0}^{\left[\rho, \rho^{\prime}\right)^{\psi=0}}$ par $\tau-1$. Pour

$$
g \in \varphi(x)^{b} \mathcal{H}_{0}^{\left[\rho, \rho^{\prime}\right)^{\psi=0}} \text { et } h \in \varphi(x)^{a} \mathcal{H}_{0}^{\left[\rho, \rho^{\prime}\right)^{\psi=0}}
$$

posons $F_{g}(h)=h-\mu((\tau-1) h-g)$. L'équation $F_{g}(h)=h$ est équivalente à $(\tau-1) h=g$. On a

$$
\begin{aligned}
F_{g}(h) & =h-\mu((\tau-1) h)-\mu(g) \\
& \in\left(\varphi(x)^{a+p^{m-1}(p-1)-1}, \varphi(x)^{b-p^{m-1}}\right) \mathcal{H}_{0}^{\left[\rho, \rho^{\prime}\right)^{\psi=0}} \\
& \subset \varphi(x)^{a} \mathcal{H}_{0}^{\left[\rho, \rho^{\prime}\right)} \psi=0
\end{aligned}
$$

pour $b \geqslant a+p^{m-1}\left(\operatorname{car} p^{m-1}(p-1)-1 \geqslant 1\right)$ et si $h-h^{\prime} \in \varphi(x)^{d} \mathcal{H}_{0}^{\left[\rho, \rho^{\prime}\right.}{ }^{\psi=0}$,

$$
\begin{aligned}
F_{g}(h)-F_{g}\left(h^{\prime}\right) & =\left(h-h^{\prime}\right)-\mu\left((\tau-1)\left(h-h^{\prime}\right)\right) \\
& \in \varphi(x)^{d+p^{m-1}(p-1)-1} \mathcal{H}_{0}^{\left[\rho, \rho^{\prime}\right)} \psi=0 \\
& \subset \varphi(x)^{d+1} \mathcal{H}_{0}^{\left[\rho, \rho^{\prime}\right)} \psi=0
\end{aligned}
$$

Ainsi, pour $b \geqslant a+p^{m-1}$ (par exemple, $b=a+p^{m-1}$ ), $F_{g}$ est une application de $\varphi(x)^{a} \mathcal{H}_{0}^{\left[\rho, \rho^{\prime}\right)^{\psi=0}}$ dans lui-même contractante pour la topologie $\varphi(x)$-adique et admet donc un point fixe. Aussi, $\varphi(x)^{a+p^{m-1}} \mathcal{H}_{0}^{\left[\rho, \rho^{\prime}\right)^{\psi=0}}$ est contenu dans l'image de $\varphi(x)^{a} \mathcal{H}_{0}^{\left[\rho, \rho^{\prime}\right)^{\psi=0}}$ par $\tau-1$ pour tout entier $a$.

Remarques. - Remarquons que

$$
\begin{aligned}
(\tau-1)\left(\varphi(x)^{a} \varphi(g)\right) & =\varphi\left((\tau-1)\left(x^{a} g\right)\right. \\
& \subset \varphi\left(x^{a+p^{m}-1} \mathcal{H}_{0}^{\left[\rho, \rho^{\prime}\right)}\right) \subset \varphi(x)^{a+p^{m}-1} \mathcal{H}_{0}^{\left[\rho, \rho^{\prime}\right)} .
\end{aligned}
$$


Comme $p^{m}-1>p^{m-1}$,

$$
(\tau-1)\left(\varphi(x)^{a} \varphi\left(\mathcal{H}_{0}^{\left[\rho, \rho^{\prime}\right)}\right)\right) \subset \varphi(x)^{a+p^{m}-1} \mathcal{H}_{0}^{\left[\rho, \rho^{\prime}\right)} \neq \varphi(x)^{a+p^{m-1}} \mathcal{H}_{0}^{\left[\rho, \rho^{\prime}\right)} .
$$

L'image de $\varphi(x)^{a} \mathcal{H}_{0}^{\left[\rho, \rho^{\prime}\right)} \operatorname{par}(\tau-1)$ n'est donc pas égale à $\varphi(x)^{a+p^{m-1}} \mathcal{H}_{0}^{\left[\rho, \rho^{\prime}\right)}$, ce qui explique que la proposition ne s'étende pas à tout $\mathcal{H}^{\left[\rho, \rho^{\prime}\right)^{\psi=0}}$.

B.2.6. Corollaire. - Pour tout entier $k \in \mathbb{Z}$ et pour $\rho>\rho_{m}$,

$$
(\tau-1)^{k}\left(\mathcal{H}_{0}^{\left[\rho, \rho^{\prime}\right)}{ }^{\psi=0}\right) \subset \begin{cases}\varphi(x)^{k p^{m-1}} \mathcal{H}_{0}^{\left[\rho, \rho^{\prime}\right)^{\psi=0}} & \text { si } k<0 \\ \varphi(x)^{k\left(p^{m-1}-1\right)} \mathcal{H}_{0}^{\left[\rho, \rho^{\prime}\right)^{\psi=0}} \text { ou } x^{k\left(p^{m}-1\right)} \mathcal{H}_{0}^{\left[\rho, \rho^{\prime}\right)} & \text { si } k \geqslant 0 .\end{cases}
$$

Pour $f \in \mathcal{H}^{[\rho, 1[}$ et $\rho \leqslant r<1$ et $k \in \mathbb{Z}$

$$
\left\|(\tau-1)^{k}(f)\right\|_{r} \leqslant \begin{cases}r^{k p^{m}}\|f\|_{r} & \text { si } k<0 \\ r^{k\left(p^{m}-1\right)}\|f\|_{r} & \text { si } k \geqslant 0 .\end{cases}
$$

B.2.7. Proposition. - Soit $\rho>\rho_{m}$. Soit $\lambda$ un élément de $\mathcal{B}\left(G_{\infty}\right): \lambda=f(\tau-1)$ avec $f=\sum_{k \in \mathbb{Z}} a_{k} x^{k} \in \mathcal{H}^{[\rho, 1\}}$. Soit $U \in \mathcal{H}_{0}^{\left[\rho^{\prime}, 1\right)^{\psi=0}}$, alors $\sum_{k \in \mathbb{Z}} a_{k}(\tau-1)^{k}(U)$ définit un élément de $\mathcal{H}^{\left[\rho^{\prime \prime}, 1\right\}^{\psi=0}}$ avec $\rho^{\prime \prime}=\sup \left(\rho^{\prime}, \rho^{1 / p^{m}}\right)$.

Démonstration. - Par hypothèse, pour tout $\rho \leqslant r<1, \lim _{k \rightarrow \pm \infty}\left|a_{k}\right| r^{k}=0$. Soit $U \in \mathcal{H}^{\left[\rho^{\prime}, 1\left[{ }^{\psi=0}\right.\right.}$. Prenons $r<1$ supérieur à $\rho^{\prime \prime}=\sup \left(\rho^{\prime}, \rho^{1 / p^{m}}\right)$. On a

$$
\left\|a_{k}(\tau-1)^{k}(U)\right\|_{r} \leqslant \begin{cases}\left|a_{k}\right| r^{\left(p^{m}-1\right) k}\|U\|_{r} & \text { si } k \geqslant 0 \\ \left|a_{k}\right| r^{p^{m} k}\|U\|_{r} & \text { si } k<0 .\end{cases}
$$

Comme $r^{p^{m}-1} \geqslant r^{p^{m}} \geqslant \rho$, on en déduit que

$$
\lim _{k \rightarrow \pm \infty}\left\|a_{k}(\tau-1)^{k}(U)\right\|_{r}=0 ;
$$

plus précisément pour $\lambda \in \mathcal{H}^{[\rho, 1[}\left(G_{\infty}\right)$, pour $U \in \mathcal{H}^{\left[\rho^{\prime}, 1[\right.}$ et pour tout $r$ tel que $\rho^{\prime \prime} \leqslant$ $r<1$

$$
\|\lambda \cdot U\|_{r} \leqslant \sup \left(\|\lambda\|_{r^{p^{m}}},\|\lambda\|_{r^{p^{m}-1}}\right)\|U\|_{r}
$$

B.2.8. Corollaire. - L'application continue $\mathcal{B}\left(G_{\infty}\right) \rightarrow \mathcal{B}^{\psi=0}$ définie par

$$
\lambda \longmapsto \lambda \cdot(1+x)
$$

est une application linéaire bijective; l'image de $\mathcal{H}\left(G_{\infty}\right)$ est $\mathcal{H}^{\psi=0}$.

Démonstration. - Montrons d'abord que $\left(x^{-a} \mathbb{Z}_{p} \llbracket x \rrbracket\right)^{\psi=0} \subset \mathcal{B}\left(G_{\infty}\right)(1+x)$. Pour cela, on remarque que pour $G \in\left(x^{-a} \mathbb{Z}_{p} \llbracket x \rrbracket\right)^{\psi=0}$,

$$
(\tau-1)^{k}(G) \in\left(x^{-a+k\left(p^{m}-1\right)} \mathbb{Z}_{p} \llbracket x \rrbracket\right)^{\psi=0}
$$

et donc que, pour $k \geqslant a /\left(p^{m}-1\right)$,

$$
(\tau-1)^{k}(G) \in \mathbb{Z}_{p} \llbracket x \rrbracket^{\psi=0}=\mathbb{Z}_{p} \llbracket G_{\infty} \rrbracket(1+x) .
$$


On en déduit que $G$ appartient à $\mathcal{B}\left(G_{\infty}\right)(1+x)$.

Soit maintenant $G \in \mathcal{H}_{0}^{\left[\rho, \rho^{\prime}\right)^{\psi=0}}$. Pour tout entier $n$, il existe un entier $b_{n}$ tel que

$$
G \in p^{n} \mathcal{H}_{0}^{\left[\rho, \rho^{\prime}\right)^{\psi=0}}+\left(x^{-b_{n}} \mathbb{Z}_{p} \llbracket x \rrbracket\right)^{\psi=0} .
$$

Donc $G \in p^{n} \mathcal{H}_{0}^{\left[\rho, \rho^{\prime}\right)^{\psi=0}}+\mathcal{B}\left(G_{\infty}\right)(1+x)$ pour tout entier $n$, ce que implique que $G \in \mathcal{B}\left(G_{\infty}\right)(1+x)$, puisque $\mathcal{H}_{0}^{\left[\rho, \rho^{\prime}\right)}$ est complet pour la topologie $p$-adique et que les $p^{n} \mathcal{H}_{0}^{\left[\rho, \rho^{\prime}\right)}$ forment une base de voisinages. 


\section{QUELQUES FORMULES}

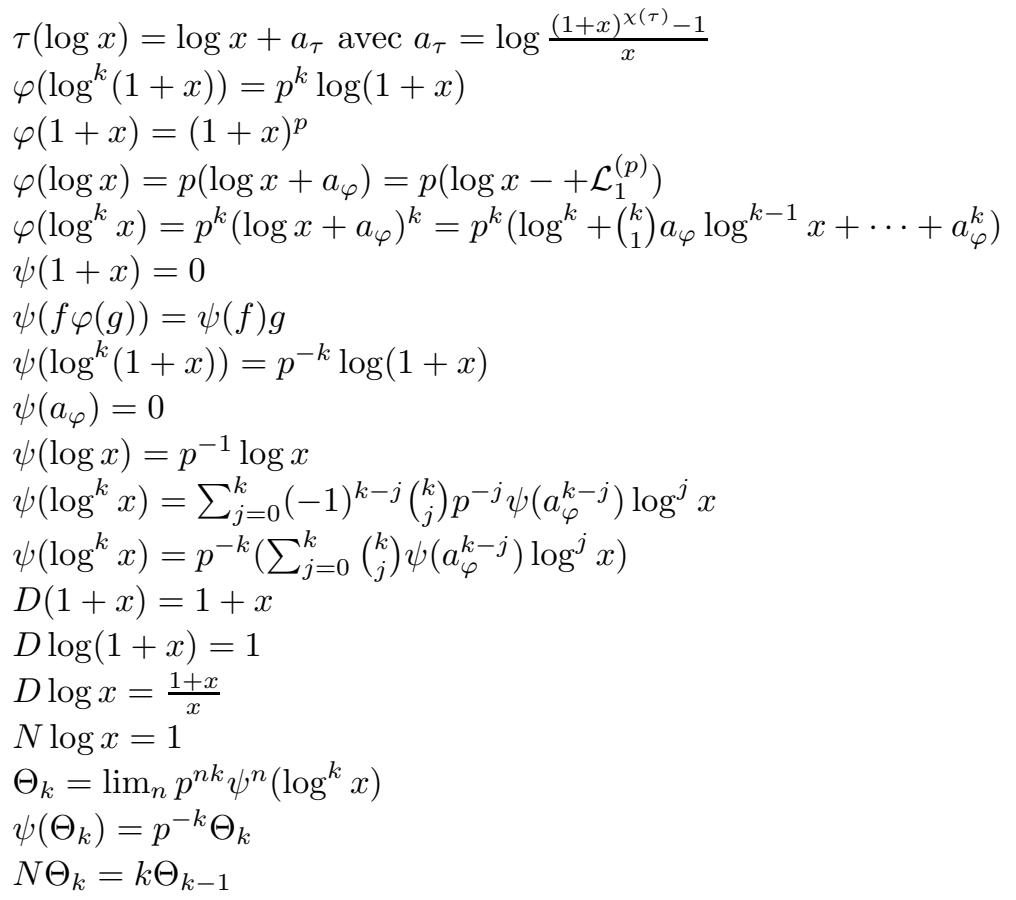





\section{BIBLIOGRAPHIE}

[1] Y. Amice, Les nombres p-adiques, PUF, Paris, 1975.

[2] S. Bloch et K. Kato, L-functions and Tamagawa numbers of motives, dans The Grothendieck Festschrift, Vol. I, pp. 333-400, Birkhäuser, Boston, 1990.

[3] F. Cherbonnier et P. Colmez, Représentations p-adiques surconvergentes, Invent. Math. 133 (1998), 581-611.

[4] - Théorie d'Iwasawa des représentations p-adiques d'un corps local, J. Am. Math. Soc 12 (1999), 241-268.

[5] R. F. Coleman, Dilogarithms, regulators and p-adic L-functions, Invent. Math. 69 (1982), 171-208.

[6] P. Colmez, Théorie d'Iwasawa des représentations de de Rham, Annals of Math. 148 (1998), 485-571.

[7] _ Représentations cristallines et représentations de hauteur finie, J. Reine Angew. Math. 514 (1999), 119-143.

[8] _ Fonctions L p-adiques, Séminaire Bourbaki, Vol. 1998/99, Astérisque 266 (2000) Exp. No. 851, 3, 21-58.

[9] B. Dwork, G. Gerotto et F. J. Sullivan, An introduction to G-functions, Annals of Math. Studies 133 (1994), Princeton University Press.

[10] J.-M. Fontaine, Modules galoisiens, modules filtrés et anneaux de Barsotti-Tate, dans Journées de Géométrie algébrique de Rennes (III), Astérisque 65 (1979), 3-80.

[11] J.-M. Fontaine et B. Mazur, Geometric Galois representations, dans Elliptic curves, modular forms, et Fermat's last theorem (Hong Kong) Internat. Press, Cambridge (1993), pp. 41-78. 
[12] J.-M. Fontaine et B. Perrin-Riou, Autour des conjectures de Bloch et Kato : cohomologie galoisienne et valeurs de fonctions L, dans Motives (Seattle), Proceedings of Symposia in Pure Mathematics, vol. 55, part 1 (1994), pp. 599-706.

[13] K. Kato, Lectures on the approach to Iwasawa theory for Hasse-Weil functions via $B_{\mathrm{dR}}$, dans Arithmetic Algebraic Geometry, Lecture Notes in Math. 1553 (1993), 50-63.

[14] N. Koblitz, p-adic analysis : a short course on recent work, Cambridge University Press, 1980.

[15] J. Nekovář. On p-adic height pairings, dans Séminaire de Théorie des Nombres, Paris, 1990-91 (1993), pp. 127-202. Birkhäuser Boston, Boston, MA,

[16] F. Knudsen et D. Mumford, The projectivity of the moduli space of stable curves. I. Preliminaries on "det" and "Div", Math. Scand. 39 (1976), 19-55.

[17] B. Perrin-Riou, Théorie d'Iwasawa p-adique locale et globale, Invent. Math. 99 (1990), 247-292.

[18] $137-185$.

[19] Théorie d'Iwasawa des représentations p-adiques sur un corps local, Invent. Math. 115 (1994), 81-149.

[20] _ La fonction de Kubota-Leopoldt, Contemp. Math. 174 (1994), 65-93.

[21] _ Fonctions L p-adiques et représentations p-adiques, Astérisque 229, Paris, 1995. Traduction : $p$-adic $L$-functions and $p$-adic representations, American Mathematical Society, Providence, RI, (2000).

[22] Fonctions L p-adiques d'une courbe elliptique et points rationnels, Ann. Inst. Fourier, 43, (1993), 945-995.

[23] Mathematicians, Vol. 1, 2 (Zürich, 1994), Birkhäuser, Basel (1995) pp. 400-410,

[24] Zéros triviaux des fonctions L p-adiques : un cas particulier, Compos. Math. 114 (1998), 37-76.

[25] _ Théorie d'Iwasawa et loi explicite de réciprocité, Doc. Math. 4 (1999), $215-269$

[26] _ Représentations p-adiques et normes universelles : I. Le cas cristallin, Prépublications d'Orsay 98-65 (1998), J. Am. Math. Soc. 13, (2000), 533-551.

[27] _ Quelques remarques sur la théorie d'Iwasawa des courbes elliptiques, prépublication 2000 .

[28] B. Totaro, Tensor products in p-adic Hodge theory, Duke Math. J. 83 (1996), 79-104. 


\section{INDEX}

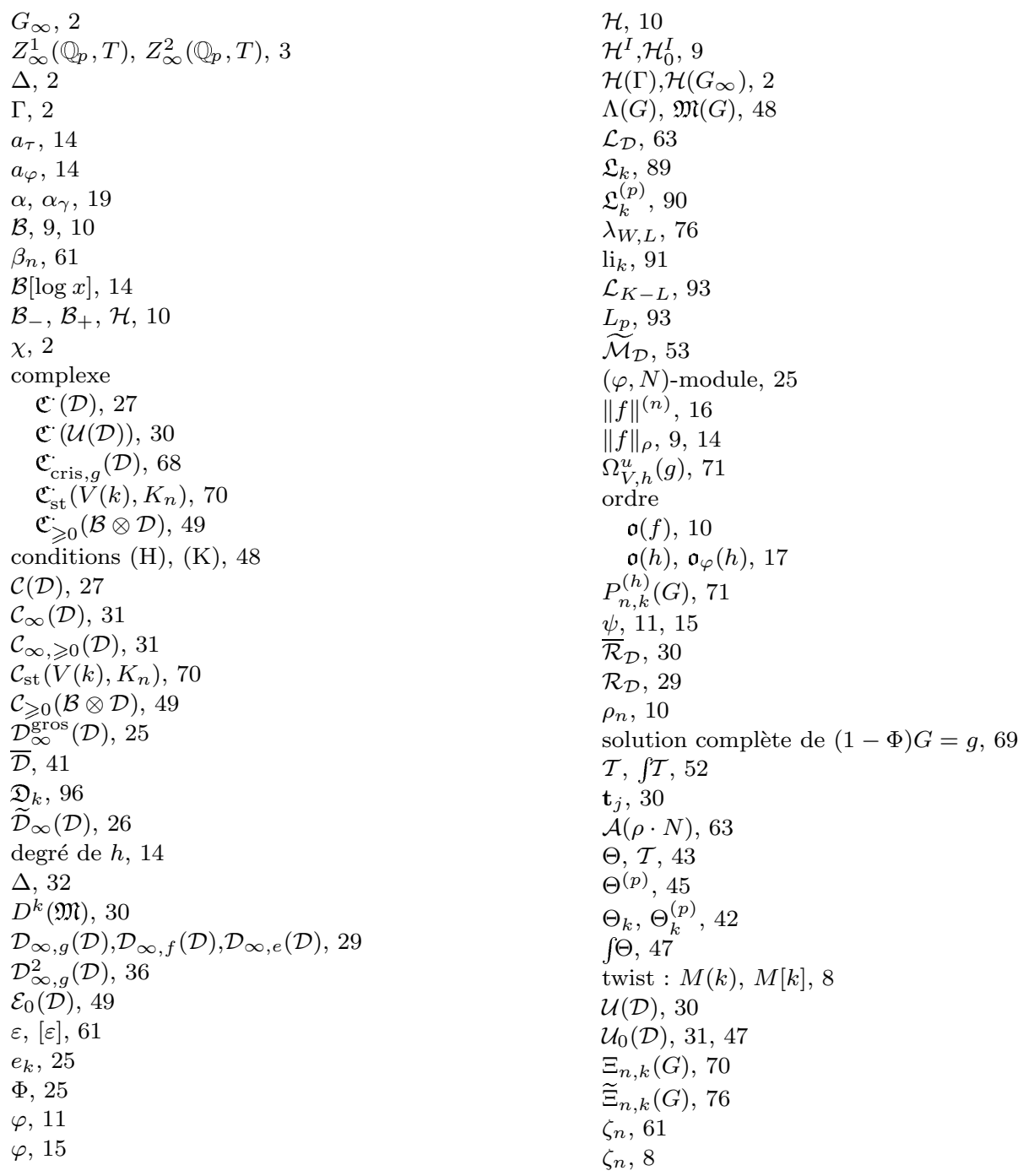

